Método da média para equações diferenciais funcionais retardadas impulsivas via equações diferenciais generalizadas 
SERVIÇO DE PÓS-GRADUAÇÃO DO ICMC-USP

Data de Depósito: 14/08/2009

Assinatura:

\section{Método da média para equações diferenciais funcionais retardadas impulsivas via equações diferenciais generalizadas}

\section{Jaqueline Bezerra Godoy}

Orientadora: Profa. Dra. Márcia Cristina Anderson Braz Federson

Dissertação apresentada ao Instituto de Ciências Matemáticas e de Computação - ICMC-USP, como parte dos requisitos para obtenção do título de Mestre em Ciências - Matemática.

USP - São Carlos

Agosto/2009 


\section{Agradecimentos}

Inicialmente, gostaria de agradecer a Sir. Sathya Sai Baba por ter me dado forças para superar os obstáculos que encontrei ao longo desta caminhada e por tudo que conquistei.

Agradeço à minha família por todo apoio dado. Em especial à minha mãe, Maria Aparecida Bezerra Godoy, por sempre ter acreditado em mim, me apoiado e ajudado a concluir mais essa importante etapa da minha vida; e ao meu pai, Gilson Godoy, por toda confiança, carinho e apoio dado durante o mestrado. Também gostaria de agradecer à minha irmã, Cynthia Bezerra Godoy, que sempre esteve ao meu lado, confiou em mim e me incentivou com relação aos meus estudos. Também agradeço aos meus segundos pais, Márcio Alves Zuany e Elaine Dantas Godoy por sempre terem acreditado em mim.

Gostaria de agradecer ao meu namorado, amigo e confidente, Luís Góes Mesquita, por ter estado ao meu lado nesses últimos tempos, me apoiando, compreendendo e ajudando, assim como pela sua paciência, carinho e atenção. Amo-te muito!

Gostaria de agradecer aos amigos que fiz nesta jornada até aqui. Em especial à Iris de Oliveira, Renato Alejandro e Pedro Henrique Apoliano, que sempre estiveram ao meu lado, dando apoio e confiança. Sem eles, talvez não tivesse conseguido concluir esta etapa da minha vida. Também gostaria de agradecer às amigas Laura Cristina Lobato, Lívia Andréia Gomes, Roberta Pacheco e Jéssica Miranda, e aos amigos Ismael Sobrinho e Rodrigo Noleto por todo apoio, amizade e confiança.

À FAPESP, pelo apoio financeiro para realização deste trabalho.

Finalmente, agradeço à minha orientadora, professora Dra. Márcia Federson, pela paciência, amizade, compreensão e confiança, bem como por todo seu incentivo e empenho dedicado ao desenvolvimento deste trabalho, por meio das diversas leituras minuciosas de cada linha deste. 


\section{Resumo}

Neste trabalho, nós consideramos o seguinte problema de valor inicial para uma equação diferencial funcional retardada com impulsos

$$
\left\{\begin{array}{l}
\dot{x}=\varepsilon f\left(t, x_{t}\right), \quad t \neq t_{k} \\
\Delta x\left(t_{k}\right)=\varepsilon I_{k}\left(x\left(t_{k}\right)\right), k=0,1,2, \ldots \\
x_{t_{0}}=\phi
\end{array}\right.
$$

onde $f$ está definida em um aberto $\Omega$ de $\mathbb{R} \times G^{-}\left([-r, 0], \mathbb{R}^{n}\right)$ e assume valores em $\mathbb{R}^{n}$, $\varepsilon>0$ é um parâmetro pequeno e $\phi \in G^{-}\left([-r, 0], \mathbb{R}^{n}\right), r>0$, onde $G^{-}\left([-r, 0], \mathbb{R}^{n}\right)$ denota o espaço das funções de $[-r, 0]$ em $\mathbb{R}^{n}$ que são regradas e contínuas à esquerda. Além disso, $t_{0}<t_{1}<\ldots<t_{k}<\ldots$ são momentos pré determinados de impulsos tais que $\lim _{k \rightarrow+\infty} t_{k}=$ $+\infty$ e $\Delta x\left(t_{k}\right)=x\left(t_{k}^{+}\right)-x\left(t_{k}\right)$. Os operadores de impulso $I_{k}, k=0,1, \ldots$ são funções contínuas de $\mathbb{R}^{n}$ em $\mathbb{R}^{n}$. Consideramos, também, que para cada $x \in G^{-}\left([-r, \infty), \mathbb{R}^{n}\right)$, $t \mapsto f\left(t, x_{t}\right)$ é uma função localmente Lebesgue integrável e sua integral indefinida satisfaz uma condição do tipo Carathéodory. Além disso, $f$ é lipschitziana na segunda variável. Definimos

$$
f_{0}(\psi)=\lim _{T \rightarrow \infty} \frac{1}{T} \int_{t_{0}}^{T} f(t, \psi) d t \quad \text { e } \quad I_{0}(x)=\lim _{T \rightarrow \infty} \frac{1}{T} \sum_{0 \leq t_{i}<T} I_{i}(x)
$$

onde $\psi \in G^{-}\left([-r, 0], \mathbb{R}^{n}\right)$ e $x \in \mathbb{R}^{n}$, e consideremos a seguinte equação diferencial funcional autônoma "média"

$$
\left\{\begin{array}{l}
\dot{y}=\varepsilon\left[f_{0}\left(y_{t}\right)+I_{0}(y(t))\right] \\
y_{t_{0}}=\phi
\end{array}\right.
$$

Então, provamos que, sob certas condições, a solução $x(t)$ de (1) se aproxima da solução $y(t)$ de $(2)$ em tempo assintoticamente grande. 


\section{Abstract}

In this present work, we consider the following initial value problem for a retarded functional differential equation with impulses

$$
\left\{\begin{array}{l}
\dot{x}=\varepsilon f\left(t, x_{t}\right), \quad t \neq t_{k} \\
\Delta x\left(t_{k}\right)=\varepsilon I_{k}\left(x\left(t_{k}\right)\right), k=0,1,2, \ldots \\
x_{t_{0}}=\phi
\end{array}\right.
$$

where $f$ is defined in a open set $\Omega \subset \mathbb{R} \times G^{-}\left([-r, 0], \mathbb{R}^{n}\right), r>0$, and takes values in $\mathbb{R}^{n}$, $\varepsilon>0$ is a small parameter and $\phi \in G^{-}\left([-r, 0], \mathbb{R}^{n}\right)$, where $G^{-}\left([-r, 0], \mathbb{R}^{n}\right)$ denotes the space of regulated functions from $[-r, 0]$ to $\mathbb{R}^{n}$ which are left continuous. Furthermore, $t_{0}<t_{1}<\ldots<t_{k}<\ldots$ are pre-assigned moments of impulse effects such that $\lim _{k \rightarrow+\infty} t_{k}=$ $+\infty$ and $\Delta x\left(t_{k}\right)=x\left(t_{k}^{+}\right)-x\left(t_{k}\right)$. The impulse operators $I_{k}, k=0,1, \ldots$, are continuous mappings from $\mathbb{R}^{n}$ to $\mathbb{R}^{n}$. For each $x \in G^{-}\left([-r, \infty), \mathbb{R}^{n}\right), t \mapsto f\left(t, x_{t}\right)$ is locally Lebesgue integrable and its indefinite integral satisfies a Carathéodory-type condition. Moreover, $f$ is Lipschitzian with respect to the second variable. We define

$$
f_{0}(\psi)=\lim _{T \rightarrow \infty} \frac{1}{T} \int_{t_{0}}^{T} f(t, \psi) d t \quad \text { and } \quad I_{0}(x)=\lim _{T \rightarrow \infty} \frac{1}{T} \sum_{0 \leq t_{i}<T} I_{i}(x),
$$

where $\psi \in G^{-}\left([-r, 0], \mathbb{R}^{n}\right)$ and $x \in \mathbb{R}^{n}$, and we consider the "averaged" autonomous functional differential equation

$$
\left\{\begin{array}{l}
\dot{y}=\varepsilon\left[f_{0}\left(y_{t}\right)+I_{0}(y(t))\right] \\
y_{t_{0}}=\phi
\end{array}\right.
$$

Then we prove that, under certain conditions, the solution $x(t)$ of (3) approximates the solution $y(t)$ of (4) in an asymptotically large time interval. 


\section{Sumário}

Introdução 1

1 Equações Diferenciais Ordinárias Generalizadas $\quad 7$

1.1 A Integral de Kurzweil . . . . . . . . . . . . . . . . . . . . . 7

1.2 Noções básicas de EDOGs . . . . . . . . . . . . . . . . . . . . 21

1.3 Existência e unicidade de soluções . . . . . . . . . . . . . . . . . 30

1.4 Dependência contínua para EDOGs . . . . . . . . . . . . . . . . . 33

2 Equações Diferenciais Funcionais Retardadas com Impulsos 49

2.1 Noções básicas de EDFRs . . . . . . . . . . . . . . . . . . . . . 49

2.2 Noções básicas de EDIs . . . . . . . . . . . . . . . . . . . . . . . . 53

2.3 Noções básicas de EDFRIs . . . . . . . . . . . . . . . . . . . . . . . . . . 62

2.4 EDFRIs num contexto mais geral . . . . . . . . . . . . . . . 67

3 Dependência contínua para EDOGs e EDFRIs $\quad 79$

3.1 Correspondência entre EDFRIs e EDOGs . . . . . . . . . . . . . . . . . 79

3.2 Dependência contínua para EDFRIs . . . . . . . . . . . . . . . . . . 94

4 Método da Média para EDFRIs $\quad 111$

4.1 O Princípio da Média para EDOGs . . . . . . . . . . . . . . . . . . . 112

4.2 O Princípio da Média para EDIs . . . . . . . . . . . . . . . . 117

4.3 O Princípio da Média para EDFRs . . . . . . . . . . . . . 118

4.4 O Princípio da Média para EDFRIs . . . . . . . . . . . . . . . . . 127

Referências $\quad 133$ 



\section{Introdução}

Um problema clássico que vem sendo estudado há muito tempo é o problema dos três corpos em mecânica celeste. Este problema trata do movimento de dois planetas ao redor de um grande sol e um parâmetro pequeno dado pela razão entre a massa de um dos planetas e a massa do sol.

Se não levarmos em consideração a interação entre os planetas, então a trajetória de cada planeta pode ser descrita por uma elipse. Em nosso sistema solar, Júpiter e Saturno possuem massa bem maior do que as dos outros planetas. No problema dos três corpos, considerando-se estes dois planetas e nosso sol, o parâmetro pequeno é da ordem $10^{-3}$ a $10^{-4}$. Além disso, em nosso sistema solar, as excentricidades e as inclinações das órbitas são pequenas (da ordem de $10^{-1}$ ).

Se levarmos em consideração a interação entre os planetas, então o movimento dos planetas poderá ser muito mais complicado. Nesta situação, o movimento dos planetas se aproxima de uma órbita elíptica e os parâmetros do movimento sofrem pequenas variações. Tal sistema é caracterizado por duas frequências que são as frequências de rotação de cada planeta ao redor do sol. Assim, para tratar do problema dos três corpos, desenvolveu-se a teoria das perturbações.

O problema dos três corpos foi estudado, por exemplo, em trabalhos do matemático, físico e filosófo francês Henri Poincaré, onde a estabilidade do sistema foi analisada por métodos da teoria das perturbações. A principal dificuldade na teoria das perturbações é causada por pequenas frequências de ressonância. O desaparecimento de uma frequência num sistema com várias frequências (sistema multifrequência) é chamado de ressonância, ou seja, é a tendência de uma frequência de oscilar em máxima amplitude, também chamada de frequência ressonante.

Além do problema clássico dos três corpos, a classe de problemas de ressonância inclui, por exemplo, o problema de estabilidade do movimento de um satélite com respeito ao seu centro de massa: deve-se assegurar movimentos ressonantes estáveis do giroscópio que fica 
suspenso livremente. Estes sistemas ressonantes são usualmente modelados por equações diferenciais ordinárias com perturbações.

Outras aplicações nesta direção podem ser encontradas em oscilações não lineares, controle de barulho, análise de estabilidade, teoria de bifurcação, controle de vibrações, entre outras.

Começando pelo matemático, astrônomo e físico alemão Johann Carl Friedrich Gauss, diversos pesquisadores notaram que o lado direito das equações que modelam problemas de mecânica celeste possuem termos que oscilam rapidamente e termos que variam de forma mais lenta. Os termos que variam lentamente determinam a evolução lenta dos parâmetros do sistema. Por outro lado, os termos que oscilam rapidamente afetam muito pouco o movimento e, portanto, podem ser omitidos. E o processo de se omitir os termos que oscilam rapidamente no lado direito das equações é chamado método da média. Assim, métodos da média ou "sistemas médios" têm sido aplicados em mecânica celeste há muito tempo para se omitir termos que oscilam rapidamente do lado direito de equações.

Justificativas para o uso de métodos da média ou "sistemas médios" para sistemas não lineares quaisquer foram apresentadas pela primeira vez nos trabalhos de N. N. Bogolyubov e A. Mitropolskii (veja [4]) e de N. N. Krylov e N. N. Bogolyubov (veja [17]), quando a descrição dos sistemas na forma usualmente conhecida foi introduzida:

$$
\left\{\begin{array}{l}
\dot{x}=\varepsilon X(x, t) \\
x(0)=x_{0}
\end{array}\right.
$$

onde $\varepsilon$ é um parâmetro pequeno e $x$ e $X$ são vetores $n$-dimensionais.

Para o sistema (5), considera-se o seguinte "sistema médio"

$$
\left\{\begin{array}{l}
\dot{y}=\varepsilon X_{0}(y) \\
y(0)=x_{0}
\end{array}\right.
$$

onde o lado direito da equação (6) é obtido tomando-se uma média do lado direito do sistema (5), ou seja,

$$
X_{0}(x)=\lim _{T \rightarrow \infty} \frac{1}{T} \int_{0}^{T} X(t, x) d t .
$$

É evidente que o sistema (6) é muito mais simples do que o sistema inicial (5). Além disso, (6) é um sistema autônomo.

O primeiro resultado provado para esta teoria, conhecido como Princípio da Média, diz que as soluções de (5) e (6) estarão próximas uma da outra em tempo assintoticamente 
grande $\left(t \in\left[0, \frac{L}{\varepsilon}\right]\right)$, desde que o sistema (5) tenha uma solução e o lado direito de (5) satisfaça uma condição de Lipschitz na segunda variável. Fica claro, portanto, que o objetivo do método da média ou Princípio da Média é determinar condições sob quais a solução do sistema (5), que admite um parâmtro pequeno, pode ser aproximada por uma solução de um sistema diferencial ordinário autônomo (como (6), por exemplo).

A teoria do método da média para equações diferenciais ordinárias (EDOs) está bem desenvolvida. A teoria do método da média para equações diferenciais funcionais (EDFs) também tem sido desenvolvida em artigos relativamente recentes. Nos anos 60, por exemplo, autores como V. I. Foduck [8], A. Halanay [12], J. K. Hale [14], G. N. Medvedev [24] e V. M. Volosov [28] desenvolveram métodos da média para certas EDFs com parâmetro pequeno, podendo estas serem aproximadas por EDOs autônomas.

A partir dos anos 70, passou-se a pensar que "sistemas médios" de EDFs com parâmetro pequeno deveriam ser EDFs autônomas em lugar da aproximação clássica por EDOs autônomas. Desta forma, seria possível manter a natureza infinito-dimensional do "sistema médio", e como consequência, a aproximação do sistema inicial seria melhor. De fato, isto pode ser verificado por simulações computacionais. Por exemplo, V. Strygin em [27] e B. Lehman e S. P. Weibel em [22], consideraram os "sistemas médios" de EDFs com parâmetro pequeno como sendo EDFs autônomas. Mais precisamente, eles consideraram a $\mathrm{EDF}$

$$
\left\{\begin{array}{l}
\dot{x}=\varepsilon f\left(t, x_{t}\right) \\
x_{0}=\phi,
\end{array}\right.
$$

onde $\varepsilon>0$ é um parâmetro pequeno e $x_{t}(\theta)=x(t+\theta)$, para $\theta \in[-r, 0]$, com $r \geq 0$ e $t \geq 0$, e a função inicial $\phi$ é um elemento do espaço de Banach $\mathcal{C}=\mathcal{C}\left([-r, 0], \mathbb{R}^{n}\right)$ das funções contínuas de $[-r, 0]$ em $\mathbb{R}^{n}$, munido da norma usual do supremo. Também considerou-se que $f$ é uma função contínua de $\mathbb{R} \times \mathcal{C}$ em $\mathbb{R}^{n}$ que é lipschitziana na segunda variável e que, para toda $\varphi \in \mathcal{C}$, o limite

$$
f_{0}(\varphi)=\lim _{T \rightarrow \infty} \frac{1}{T} \int_{0}^{T} f(s, \varphi) d s
$$

Considerou-se, também, o seguinte "sistema médio"

$$
\left\{\begin{array}{l}
\dot{y}=\varepsilon f_{0}\left(y_{t}\right) \\
y_{0}=\phi .
\end{array}\right.
$$

O Princípio da Média para (7) e (8) consistiu, portanto, em estabelecer condições sob as quais, para $\varepsilon$ suficientemente pequeno, a solução de (7) estará suficientemente próxima 
da solução de (8) em tempo finito $t \in\left[0, \frac{L}{\varepsilon}\right]$.

Em [3], D. D. Bainov e S. D. Milusheva consideraram uma equação diferencial funcional do tipo neutro dada por

$$
\left\{\begin{array}{l}
\dot{x}=\varepsilon X(t, x(t), x(\Delta(t, x(t)), \dot{x}(\Delta(t, x(t))))), \quad t>0, t \neq \tau_{i}(x), \\
x(t)=\phi(t, \varepsilon), \quad t \in[-r, 0] \\
\dot{x}(t)=\dot{\phi}(t, \varepsilon), \quad t \in[-r, 0]
\end{array}\right.
$$

onde $\varepsilon>0$ é um parâmetro pequeno, $r>0, t-r \leq \Delta(t, x(t)) \leq t, t \geq 0$ e $\phi(t, \varepsilon) \in$ $D \subset \mathbb{R}^{n}$ é a função inicial definida em $[-r, 0]$. Eles consideraram as superfícies $\tau_{i}(x)$ tais que $\tau_{i}(x)<\tau_{i+1}(x), i=1,2, \ldots$, e $\tau_{i}(x)$ pertence ao semi-espaço $t>0$ para $x \in D$ e $i=1,2, \ldots$. Consideraram, também, os seguintes impulsos

$$
x_{i}^{+}=x_{i}^{-}+\varepsilon I_{i}\left(x_{i}^{-}\right), i=1,2, \ldots
$$

que uma solução de (9) sofre quando ela encontra a superfície $\tau_{i}, i=1,2, \ldots$.

Suponhamos que os limites

$$
\lim _{T \rightarrow \infty} \frac{1}{T} \int_{t}^{t+T} X(s, x, x, 0) d s=X_{0}(x) \text { e } \lim _{T \rightarrow \infty} \frac{1}{T} \sum_{t<t_{i}<t+T} I_{i}(x)=I_{0}(x)
$$

existam e consideremos o seguinte "sistema médio" para (9)

$$
\left\{\begin{array}{l}
\dot{y}=\varepsilon X_{0}(y)+\varepsilon I_{0}(y) \\
y(0)=x_{0}
\end{array}\right.
$$

que é, na verdade, uma "EDO média" e não uma "EDF média". Milusheva e Bainov assumiram que $X(t, x, y, z)$ é contínua em $\left\{t \geq 0, x, y \in D, z \in D_{1} \subset \mathbb{R}^{n}\right\}$, que $\Delta(t, x)$ é contínua em $\{t \geq 0, x \in D\}$, que $\phi(t, \varepsilon)$ é continuamente diferenciável em $\{t \in[-r, 0], \varepsilon \in$ $(0, E]\}$ e que os operadores de impulsos $I_{i}(x), i=1,2, \ldots$, são contínuos em $D$. Eles também assumiram que as funções $\tau_{i}(x), i=1,2, \ldots$, são duas vezes continuamente diferenciáveis em $D$, e provaram que, sob determinadas condições, para cada $\mu>0$ e $L>0$, existem $\varepsilon_{0} \in(0, E], \varepsilon_{0}=\varepsilon_{0}(\eta, L)$, tal que se $0<\varepsilon \leq \varepsilon_{0}$, então $\|x(t)-y(t)\|<\eta$ para $t \in\left[0, \frac{L}{\varepsilon}\right]$, onde $x$ é solução de (9)-(10) e $y$ é solução de (11).

Note que, quando $\Delta(t, x(t))=x(t-\sigma)$, para um $\sigma>0$ fixo, então (9) é uma equação diferencial diferença do tipo neutro. 
Nesta dissertação, vamos considerar a seguinte EDF

$$
\left\{\begin{array}{l}
\dot{x}=\varepsilon f\left(t, x_{t}\right), \quad t \neq t_{k} \\
x_{0}=\phi
\end{array}\right.
$$

onde $\varepsilon>0$ é um parâmetro pequeno, a função inicial $\phi$ é regrada e contínua à esquerda, definida em $[-r, 0]$, com $r>0, x$ é uma função de $[-r,+\infty)$ em $\mathbb{R}^{n}$, para cada solução $x$ de (12), a função $t \mapsto f\left(t, x_{t}\right)$ é localmente Lebesgue integrável e sua integral indefinida satisfaz uma condição do tipo Carathéodory. Além disso, $f$ é lipschitziana na segunda variável.

Note que as condições sobre a integral indefinida e não sobre a função $f$ propriamente dita, permitem que $f$ tenha muitas descontinuidades como função da primeira variável e até possa "comportar-se mal", contanto que sua integral indefinida seja "boa" o suficiente.

Também consideraremos os tempos de impulsos $t_{0} \leq t_{1} \leq \ldots \leq t_{k} \leq \ldots$, onde $\lim _{k \rightarrow \infty} t_{k}=\infty$, e assumiremos que os operadores de impulso $I_{k}(x), k=0,1,2, \ldots$ sejam funções contínuas de $\mathbb{R}^{n}$ em $\mathbb{R}^{n}$. Consideraremos (12) sujeita às condições de impulso

$$
\Delta x\left(t_{k}\right)=x\left(t_{k}+\right)-x\left(t_{k}-\right)=x\left(t_{k}+\right)-x\left(t_{k}\right)=I_{k}\left(x\left(t_{k}\right)\right), \quad k=0,1,2, \ldots
$$

ou seja, assumimos que $x$ é contínua à esquerda.

O "sistema médio" relativo ao sistema (12)-(13) será dado por

$$
\left\{\begin{array}{l}
\dot{y}=\varepsilon f_{0}\left(y_{t}\right)+\varepsilon I_{0}(y) \\
y_{0}=\phi
\end{array}\right.
$$

onde

$$
f_{0}(\varphi)=\lim _{T \rightarrow \infty} \frac{1}{T} \int_{0}^{T} f(s, \varphi) d s \quad \text { e } \quad I_{0}(y)=\lim _{T \rightarrow \infty} \sum_{0 \leq t_{i}<T} I_{i}(y) .
$$

Neste trabalho, nós estabelecemos um resultado do tipo "Princípio da Média" para a EDF impulsiva (12)-(13). Este resultado é inédito e diz que, sob certas condições, dados $\mu>0$ e $L>0,\|x(t)-y(t)\|<\mu$, para $t \in\left[0, \frac{L}{\varepsilon}\right]$, onde $x$ é solução de (12)-(13) e $y$ é solução de (14). Tal resultado está descrito em nosso artigo [11].

A maneira pela qual obtivemos nosso resultado foi explorando o método da média de-

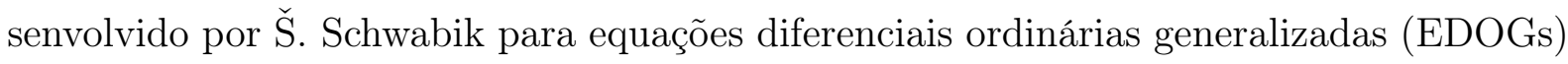
[26]. Para isto, tivemos que adaptar toda a teoria de EDOGs feita por Schwabik para funções a valores em $\mathbb{R}^{n}$ para o caso em que as funções assumem valores em um espaço de Banach $X$. Porém, nem todos os resultados foram adaptados facilmente. Em certo mo- 
mento, tivemos que restringir o espaço $X$ ao espaço das funções regradas para obtermos um resultado sobre dependência contínua de soluções de EDOGs com respeito aos valores iniciais que foi fundamental para a obtenção do resultado principal deste trabalho final. Esta restrição, entretanto, não afetou nosso objetivo que era o de estabelecer um Princípio da Média para equações diferenciais funcionais retardadas com impulsos (EDFRIs), pois, afinal, num contexto geral, as soluções de EDFRIs são mesmo funções regradas. E o contexto no qual trabalhamos trata exatamente deste tipo de solução (de variação limitada e contínua à esquerda).

É claro que outro fato fundamental que utilizamos neste trabalho é correspondência biunívoca que existe entre certa classe de EDFRIs e uma classe de EDOGs. Para isto, utilizamos os resultados de M. Federson e ̌̌ Schwabik descritos em [7].

Um terceiro aspecto fundamental deste trabalho foi a adaptação dos resultados de [19]. Neste artigo, M. Lakrib estabeleceu um Princípio da Média para EDFRs sem impulsos através da teoria da Análise Não Standard. Neste sentido, tivemos que adaptar os resultados de Lakrib usando somente a Análise Clássica.

Todos os aspectos acima serão desenvolvidos neste trabalho de forma que o leitor possa se ater à leitura desta dissertação, com poucas incursões para literaturas adicionais. Apresentaremos as teorias básicas de EDOGs, de EDFRs, de EDIs e de EDFRIs que são os pilares do nosso trabalho.

Organizamos nossa exposição da seguinte maneira: no Capítulo 1, apresentamos a teoria de EDOGs, enunciando e demonstrando alguns resultados essenciais para o nosso trabalho. No Capítulo 2, apresentamos as teorias fundamentais de EDFRs e EDIs. Apresentamos, também, vários exemplos de sistemas de EDIs. Ainda neste capítulo, apresentamos a teoria de EDFRIs, incluindo alguns resultados novos que fizemos. No Capítulo 3, apresentamos a relação entre a teoria de EDOGs e a teoria de EDFRIs. Também incluimos alguns resultados novos. Por fim, no último capítulo, apresentamos um resultado inédito sobre método da média para EDFRIs, que é o resultado principal do nosso trabalho. Ao longo deste último capítulo, entretanto, também apresentamos versões do Princípio da Média para EDOGs, EDIs (em particular, para EDOs) e para EDFRs. Todas estas versões também são inéditas e generalizam, cada uma, os resultados existentes correspondentes na literatura.

Convém mencionarmos aqui que nosso trabalho trata-se de uma justificativa para o método da média para EDFRIs. Fica, portanto, para uma próxima etapa, o tratamento de aplicações provenientes deste resultado. Tais aplicações podem incluir, por exemplo, o estudo de soluções periódicas e/ou o estudo de estabilidade para equações do tipo (12)(13). 


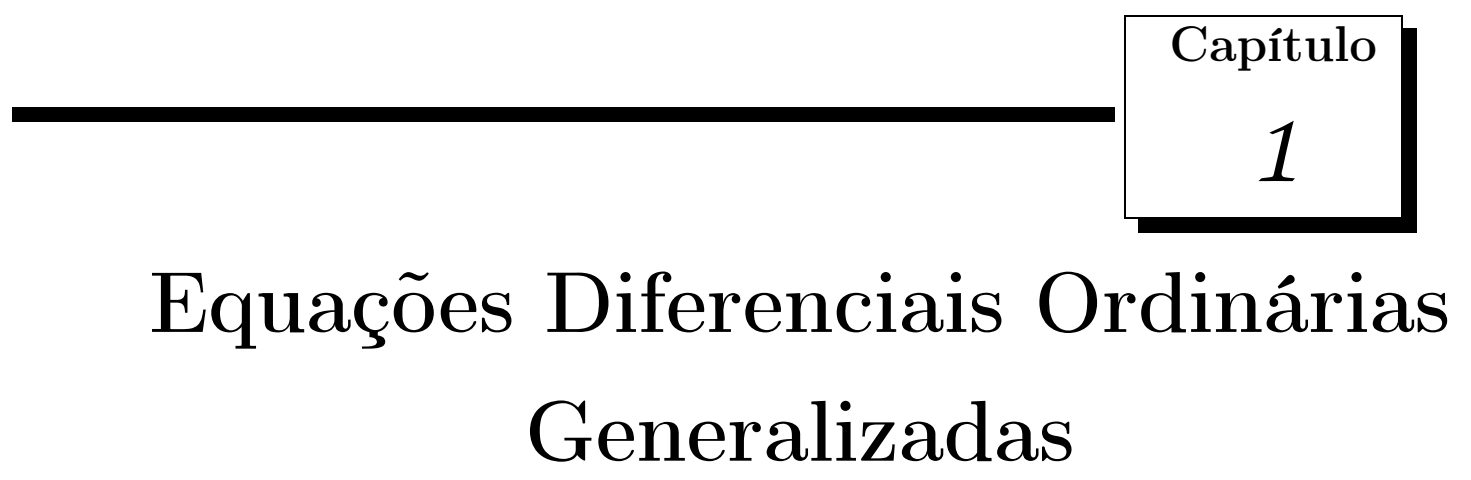

Neste capítulo, iremos introduzir algumas definições básicas e alguns resultados importantes sobre equações diferenciais ordinárias generalizadas (EDOGs) que serão úteis ao longo deste trabalho.

Este capítulo está dividido em quatro seções. Na primeira delas, abordaremos a integral de Kurzweil, apresentando a definição desta integral, suas propriedades fundamentais e alguns resultados que serão importantes ao longo deste trabalho. Na segunda seção, abordaremos as equações diferenciais ordinárias generalizadas (EDOGs), que são definidas a partir da integral de Kurzweil. Na terceira seção, analisaremos a existência e a unicidade de soluções de EDOGs. Finalmente, abordaremos a dependência contínua das soluções com respeito aos parâmetros iniciais para as EDOGs.

As principais referências para este capítulo são [26], [7] e [1].

Quando considerarmos relevante, incluiremos as demonstrações dos resultados apresentados. Vamos evitar as demonstrações excessivamente longas.

\subsection{A Integral de Kurzweil}

Seja $[a, b] \subset \mathbb{R},-\infty<a<b<+\infty$, um intervalo dado.

Dado um par $(\tau, J)$, diremos que $J$ é um intervalo marcado e $\tau$ é a marca deste intervalo, se $\tau \in \mathbb{R}$ for um ponto e $J \subset \mathbb{R}$ for um intervalo compacto. Seja $\Delta=\left\{\left(\tau_{j}, J_{j}\right), j=\right.$ $1,2, \ldots, k\}$ uma coleção finita de intervalos marcados. Diremos que esta coleção é um 
sistema em $[a, b]$, se $\tau_{j} \in J_{j} \subseteq[a, b]$ para todo $j=1,2, \ldots, k$ e $\operatorname{Int} J_{i} \bigcap \operatorname{Int} J_{j}=\emptyset$ para $j \neq i$, onde Int $J$ denota o interior de um intervalo $J$.

Diremos que um sistema $\Delta=\left\{\left(\tau_{j}, J_{j}\right), j=1,2, \ldots, k\right\}$ é uma partição de $[a, b]$, se

$$
\bigcup_{j=1}^{k} J_{j}=[a, b]
$$

Chamaremos uma função positiva $\delta:[a, b] \rightarrow(0,+\infty)$ de calibre em $[a, b]$. Assim sendo, dado um calibre $\delta$ em $[a, b]$, diremos que um intervalo marcado $(\tau, J) \operatorname{com} \tau \in[a, b]$ é $\delta$-fino, se $J \subset(\tau-\delta(\tau), \tau+\delta(\tau))$.

Um sistema (em particular, uma partição) $\Delta=\left\{\left(\tau_{j}, J_{j}\right), j=1,2, \ldots, k\right\}$ será dito $\delta$-fino, se o intervalo marcado $\left(\tau_{j}, J_{j}\right)$ for $\delta$-fino para todo $j=1,2, \ldots, k$.

Sejam $X$ um espaço de Banach e $U:[a, b] \times[a, b] \rightarrow X$ uma função de duas variáveis $\tau, t \in[a, b]$. Usaremos a notação

$$
S(U, D)=\sum_{j=1}^{k}\left[U\left(\tau_{j}, \alpha_{j}\right)-U\left(\tau_{j}, \alpha_{j-1}\right)\right]
$$

para denotar a soma de Riemman correspondente à função $U$ e à partição $D$.

O primeiro resultado que apresentamos diz que, para cada calibre $\delta$ de $[a, b]$, sempre é possível obter uma partição $\delta$-fina de $[a, b]$. Este resultado é conhecido como Lema de Cousin e é importante para a definição da integral que apresentaremos na sequência.

Lema 1.1 (Lema de Cousin - [26], Lema 1.4). Dado um calibre $\delta$ em $[a, b]$, existe uma partição $\delta$-fina $D=\left\{\alpha_{0}, \tau_{1}, \alpha_{1}, \ldots, \alpha_{k-1}, \tau_{k}, \alpha_{k}\right\}$ de $[a, b]$.

A definição de integral que apresentamos agora é devida ao matemático tcheco Jaroslav Kurzweil que generalizou a integral de Perron em seu artigo de 1957 ([18]).

Definição 1.2. Diremos que $U:[a, b] \times[a, b] \rightarrow X$ é uma função Kurzweil integrável sobre o intervalo $[a, b]$, se existir um único elemento $I \in X$ tal que para todo $\varepsilon>0$, existe um calibre $\delta$ em $[a, b]$ tal que

$$
\|S(U, D)-I\|=\left\|\sum_{j=1}^{k}\left[U\left(\tau_{j}, \alpha_{j}\right)-U\left(\tau_{j}, \alpha_{j-1}\right)\right]-I\right\|<\varepsilon
$$

para toda partição $\delta$-fina

$$
D=\left\{\left(\tau_{j},\left[\alpha_{j-1}, \alpha_{j}\right]\right), j=1, \ldots, k\right\}=\left\{\alpha_{0}, \tau_{1}, \alpha_{1}, \ldots, \tau_{k}, \alpha_{k}\right\}
$$


$d e[a, b]$.

Na definição acima, chamaremos $I$ de integral de Kurzweil de $U$ sobre o intervalo $[a, b]$ e denotaremos esta integral por $\int_{a}^{b} D U(\tau, t)$.

Se $\int_{a}^{b} D U(\tau, t)$ existir, então definiremos $\int_{a}^{b} D U(\tau, t)=-\int_{b}^{a} D U(\tau, t)$ e quando $a=b$, definiremos $\int_{a}^{b} D U(\tau, t)=0$.

Observação 1.3. Note que, na Definição 1.2, graças ao Lema de Cousin (Lema 1.1) a condição de que, dado um calibre $\delta$, sempre existe uma partição $\delta$-fina do intervalo, de fato está satisfeita (ela poderia estar satisfeita por vacuidade). Note, também, que a integral de Riemann-Stieltjes é claramente um caso particular de integral de Kurzweil quando $U(\tau, t)=f(\tau) g(t)$. Em particular, funções Riemann integráveis são integráveis no sentido de Kurzweil.

Denotaremos por $\mathcal{K}([a, b], X)$ o conjunto de todas as funções $U:[a, b] \times[a, b] \mapsto X$ que são integráveis no intervalo $[a, b]$, no sentido de Kurzweil.

A seguir, vamos enunciar e provar alguns teoremas que tratam de algumas propriedades fundamentais desta integral e que serão bastante utilizadas ao longo do nosso trabalho.

O primeiro teorema que apresentamos nos diz que a integral e Kurzweil é linear.

Teorema 1.4 (Linearidade - [26], Teorema 1.9). Se $U, V \in \mathcal{K}([a, b], X)$ e $c_{1}, c_{2} \in \mathbb{R}$, então $c_{1} U+c_{2} V \in \mathcal{K}([a, b], X) e$

$$
\int_{a}^{b} D\left[c_{1} U(\tau, t)+c_{2} V(\tau, t)\right]=c_{1} \int_{a}^{b} D U(\tau, t)+c_{2} \int_{a}^{b} D V(\tau, t) .
$$

Prova: Sejam $c_{1}, c_{2} \in \mathbb{R}$ constantes e $D$ uma partição arbitrária

$$
D=\left\{\alpha_{0}, \tau_{1}, \alpha_{1}, \ldots, \alpha_{k-1}, \tau_{k}, \alpha_{k}\right\}
$$

de $[a, b]$ e sejam $S(U, D)$ e $S(V, D)$ respectivamente as somas de Riemann das funções $U, V:[a, b] \times[a, b] \rightarrow X$. Então temos a seguinte igualdade

$$
\begin{gathered}
S\left(c_{1} U+c_{2} V, D\right)=\sum_{j=1}^{k}\left[\left(c_{1} U+c_{2} V\right)\left(\tau_{j}, \alpha_{j}\right)-\left(c_{1} U+c_{2} V\right)\left(\tau_{j}, \alpha_{j-1}\right)\right]= \\
=\sum_{j=1}^{k}\left[\left(c_{1} U\right)\left(\tau_{j}, \alpha_{j}\right)+\left(c_{2} V\right)\left(\tau_{j}, \alpha_{j}\right)-\left(c_{1} U\right)\left(\tau_{j}, \alpha_{j-1}\right)-\left(c_{2} V\right)\left(\tau_{j}, \alpha_{j-1}\right)\right]= \\
=\sum_{j=1}^{k}\left[\left(c_{1} U\right)\left(\tau_{j}, \alpha_{j}\right)-\left(c_{1} U\right)\left(\tau_{j}, \alpha_{j-1}\right)\right]+\sum_{j=1}^{k}\left[\left(c_{2} V\right)\left(\tau_{j}, \alpha_{j}\right)-\left(c_{2} V\right)\left(\tau_{j}, \alpha_{j-1}\right)\right]=
\end{gathered}
$$




$$
=c_{1} S(U, D)+c_{2} S(V, D)
$$

donde segue imediatamente o resultado.

A seguir, apresentamos o Critério de Cauchy para a integral de Kurzweil. Este critério será fundamental na demonstração do resultado seguinte.

Teorema 1.5 (Critério de Cauchy - [26], Teorema 1.7). A função $U:[a, b] \times[a, b] \rightarrow X$ será integrável sobre $[a, b]$ se, e somente se, para todo $\varepsilon>0$, existir um calibre $\delta$ em $[a, b]$ tal que

$$
\left\|S\left(U, D_{1}\right)-S\left(U, D_{2}\right)\right\|<\varepsilon
$$

para quaisquer partições $\delta$-finas $D_{1}, D_{2}$ de $[a, b]$, onde

$$
S(U, D)=\sum_{j=1}^{k}\left[U\left(\tau_{j}, \alpha_{j}\right)-U\left(\tau_{j}, \alpha_{j-1}\right)\right]
$$

é a soma de Riemann correspondente a $U$ e à partição $D=\left\{\alpha_{0}, \tau_{1}, \alpha_{1}, \ldots, \alpha_{k-1}, \tau_{k}, \alpha_{k}\right\}$ de $[a, b]$.

Prova: Para cada calibre $\delta$ de $[a, b]$, definimos

$$
\sum_{\delta}=\{S(U, D) ; D \text { é uma partição } \delta \text {-fina de }[a, b]\}
$$

Pela definição acima, fica evidente que

$$
\delta_{1} \leq \delta_{2} \Longrightarrow \sum_{\delta_{1}} \subset \sum_{\delta_{2}}
$$

Além disso, se $\delta$ for um calibre correspondente ao $\varepsilon>0$ do enunciado do teorema, então

$$
\operatorname{diam} \sum_{\delta} \leq \varepsilon
$$

Tomemos, então, uma sequência $\varepsilon_{n} \rightarrow 0$ e calibres correspondentes $\delta_{n}$, as quais podemos supor que satisfazem $\delta_{n+1} \leq \delta_{n}$. Portanto os $\sum_{\delta_{n}}$ formam uma sequência decrescente de conjuntos em $X$ com $\operatorname{diam} \sum_{\delta_{n}} \rightarrow 0$. Como $X$ é completo, então existe um e só um $I \in X$ que é aderente a todos os $\sum_{\delta_{n}}$. Desta forma, temos $\int_{a}^{b} f(t) d t=I$. De fato, pois dado $\varepsilon>0$, tomando $\varepsilon_{n}<\varepsilon$ e o calibre correspondente $\delta_{n}$ escolhido acima, então para toda partição $\delta_{n}$-fina $D$ de $[a, b]$, temos $S(U, D) \in \sum_{\delta_{n}}$ e, portanto, por $(1.1)$, vale $\|S(U, D)-I\| \leq \varepsilon_{n}<\varepsilon$ e temos o resultado desejado. 
O teorema a seguir trata da integrabilidade em subintervalos de $[a, b]$.

Teorema 1.6 ([26], Teorema 1.10). Se $U \in \mathcal{K}([a, b], X)$, então para todo $[c, d] \subset[a, b]$ teremos $U \in \mathcal{K}([c, d], X)$.

Prova: Suponhamos que $\varepsilon>0$ seja dado. Pelo Critério de Cauchy (Teorema 1.5) para a existência da integral $\int_{a}^{b} D U(\tau, t)$, existe um calibre $\delta$ em $[a, b]$ tal que

$$
\left\|S\left(U, D_{1}\right)-S\left(U, D_{2}\right)\right\|<\varepsilon
$$

para quaisquer partições $\delta$-fina $D_{1}$ e $D_{2}$ de $[a, b]$.

Agora, tomemos $\widetilde{D_{1}}$ e $\widetilde{D_{2}}$ partições $\delta$-finas arbitrárias de $[c, d]$ e suponhamos que $a<c<d<b$. Sejam $D_{L}$ uma partição $\delta$-fina de $[a, c]$ e $D_{R}$ uma partição $\delta$-fina de $[d, b]$. Estas partições existem pelo Lema de Cousin (Lema 1.1). Assim, se

$$
\begin{gathered}
\widetilde{D_{1}}=\left\{\alpha_{0}, \tau_{1}, \alpha_{1}, \ldots, \alpha_{k-1}, \tau_{k}, \alpha_{k}\right\}, \\
D_{L}=\left\{\alpha_{0}{ }^{L}, \tau_{1}{ }^{L}, \alpha_{1}{ }^{L}, \ldots, \alpha_{l-1}{ }^{L}, \tau_{l}{ }^{L}, \alpha_{l}{ }^{L}\right\}, \\
D_{R}=\left\{\alpha_{0}{ }^{R}, \tau_{1}{ }^{R}, \alpha_{1}{ }^{R}, \ldots, \alpha_{k-1}{ }^{R}, \tau_{k}{ }^{R}, \alpha_{k}{ }^{R}\right\},
\end{gathered}
$$

então $\alpha_{0}{ }^{L}=a, \alpha_{l}{ }^{L}=c=\alpha_{0}, \alpha_{r}=d=\alpha_{0}{ }^{R}$ e $\alpha_{r}{ }^{R}=b$.

Vamos unir as partições $D_{L}, \widetilde{D_{1}}, D_{R}$ para criar uma partição $D_{1}$ de $[a, b]$. Temos $D_{1}=\left\{\alpha_{0}{ }^{L}, \tau_{1}{ }^{L}, \alpha_{1}{ }^{L}, \ldots, \alpha_{l-1}{ }^{L}, \tau_{l}{ }^{L}, \alpha_{0}, \tau_{1}, \alpha_{1}, \ldots, \alpha_{k-1}, \tau_{k}, \alpha_{k}, \tau_{1}{ }^{R}, \alpha_{1}{ }^{R}, \ldots, \alpha_{r-1}{ }^{R}, \tau_{r}{ }^{R}, \alpha_{r}{ }^{R}\right\}$

Então evidentemente $D_{1}$ será uma partição de $[a, b]$ e será $\delta$-fina, pois as partições $D_{L}$, $\widetilde{D_{1}}, D_{R}$ são $\delta$-finas.

Similarmente, unindo as partições $D_{L}, \widetilde{D_{2}}, D_{R}$, obtemos uma partição $\delta$-fina $D_{2}$ de $[a, b]$. Daí, por (1.2), temos

$$
\left\|S\left(U, \widetilde{D_{1}}\right)-S\left(U, \widetilde{D_{2}}\right)\right\|=\left\|S\left(U, D_{1}\right)-S\left(U, D_{2}\right)\right\|<\varepsilon
$$

pois os termos na soma $S\left(U, D_{1}\right)-S\left(U, D_{2}\right)$ que correspondem às partes comuns de $D_{L}$ e $D_{R}$ aparecem em cada uma das partições $D_{1}$ e $D_{2}$ e, portanto, cancelam-se.

Portanto, pelo Critério de Cauchy (Teorema 1.5), a integral $\int_{c}^{d} D U(\tau, t)$ existe pois as partições $\widetilde{D_{1}}, \widetilde{D_{2}}$ são partições arbitrárias $\delta$-finas de $[c, d]$.

O teorema acima é uma generalização do Teorema 1.10 encontrado em [26], onde se fez o caso $X=\mathbb{R}^{n}$. 
O teorema abaixo é uma generalização do Teorema 1.11 encontrado em [26] e trata da aditividade da integral.

Teorema 1.7 (Aditividade - [26], Teorema 1.11). Se $c \in(a, b)$ e $U:[a, b] \times[a, b] \rightarrow X$ forem tais que $U \in \mathcal{K}([a, c], X)$ e $U \in \mathcal{K}([c, b], X)$, então $U \in \mathcal{K}([a, b], X)$ e

$$
\int_{a}^{b} D U(\tau, t)=\int_{a}^{c} D U(\tau, t)+\int_{c}^{b} D U(\tau, t) .
$$

Prova: Seja $\varepsilon>0$ dado. Sejam $I_{L}=\int_{a}^{c} D U(\tau, t)$ e $I_{R}=\int_{c}^{b} D U(\tau, t)$. Como $U \in$ $\mathcal{K}([a, c], X)$, então existe um calibre $\delta_{L}$ em $[a, c]$ tal que para toda partição $\delta_{L}$-fina $D^{L}$ de $[a, c]$, temos

$$
\left\|S\left(U, D^{L}\right)-I_{L}\right\|<\varepsilon .
$$

Analogamente, como $U \in \mathcal{K}([c, b], X)$, então existe um calibre $\delta_{R}$ em $[c, d]$ tal que para toda partição $\delta_{R}$-fina $D^{R}$ de $[c, b]$, temos

$$
\left\|S\left(U, D^{R}\right)-I_{R}\right\|<\varepsilon .
$$

Vamos definir um calibre auxiliar $\bar{\delta}$ de $[a, b]$ da seguinte forma:

$$
\bar{\delta}(\tau)=\left\{\begin{array}{l}
\delta_{L}(\tau), \text { para } \tau \in[a, c) \\
\min \left\{\delta_{L}(c), \delta_{R}(c)\right\}, \text { para } \tau=c \\
\delta_{R}(\tau), \text { para } \tau \in(c, d]
\end{array}\right.
$$

Assim, escolhemos um calibre $\delta:[a, b] \rightarrow(0,+\infty)$ de modo que

$$
\delta(\tau)<\min \{\bar{\delta}(\tau),|\tau-c|\}, \quad \text { se } \tau \neq c
$$

e

$$
\delta(c)=\bar{\delta}(c)
$$

Suponhamos que $D=\left\{\alpha_{0}, \tau_{1}, \alpha_{1}, \ldots, \alpha_{k-1}, \tau_{k}, \alpha_{k}\right\}$ seja uma partição $\delta$-fina de $[a, b]$. Então existe um índice $m$ tal que $c \in\left[\alpha_{m-1}, \alpha_{m}\right]$. Suponhamos, por contradição, que $\tau_{m} \neq c$. Então vale

$$
\left|\tau_{m}-c\right| \leq \delta\left(\tau_{m}\right)<\left|\tau_{m}-c\right|,
$$

pois pela definição de partição $\delta$-fina $\left[\alpha_{m-1}, \alpha_{m}\right] \subset\left(\tau_{m}-\delta\left(\tau_{m}\right), \tau_{m}+\delta\left(\tau_{m}\right)\right)$ e $c \in$ $\left[\alpha_{m-1}, \alpha_{m}\right]$, e também $\delta(\tau)<|\tau-c|$ para todo $\tau \neq c$. Assim, temos uma contradição. $\operatorname{Logo} \tau_{m}=c$. 
Pela definição de soma de Riemann, também temos a seguinte igualdade

$$
\begin{gathered}
S(U, D)=\sum_{j=1}^{k}\left[U\left(\tau_{j}, \alpha_{j}\right)-U\left(\tau_{j}, \alpha_{j-1}\right)\right]=\sum_{j=1}^{m-1}\left[U\left(\tau_{j}, \alpha_{j}\right)-U\left(\tau_{j}, \alpha_{j-1}\right)\right]+ \\
+U\left(\tau_{m}, \alpha_{m}\right)-U\left(\tau_{m}, \alpha_{m-1}\right)+\sum_{j=m+1}^{k}\left[U\left(\tau_{j}, \alpha_{j}\right)-U\left(\tau_{j}, \alpha_{j-1}\right)\right]= \\
=\sum_{j=1}^{m-1}\left[U\left(\tau_{j}, \alpha_{j}\right)-U\left(\tau_{j}, \alpha_{j-1}\right)\right]+U\left(c, \alpha_{m}\right)-U\left(c, \alpha_{m-1}\right)+\sum_{j=m+1}^{k}\left[U\left(\tau_{j}, \alpha_{j}\right)-U\left(\tau_{j}, \alpha_{j-1}\right)\right]= \\
=\sum_{j=1}^{m-1}\left[U\left(\tau_{j}, \alpha_{j}\right)-U\left(\tau_{j}, \alpha_{j-1}\right)\right]+U(c, c)-U\left(c, \alpha_{m-1}\right)+U\left(c, \alpha_{m}\right)-U(c, c)+ \\
+\sum_{j=m+1}^{k}\left[U\left(\tau_{j}, \alpha_{j}\right)-U\left(\tau_{j}, \alpha_{j-1}\right)\right]=S\left(U, D^{L}\right)+S\left(U, D^{R}\right),
\end{gathered}
$$

onde $D^{L}=\left\{\alpha_{0}, \tau_{1}, \alpha_{1}, \ldots, \alpha_{m-1}, \tau_{m}^{L}=c, \alpha_{m}^{L}=c\right\}$ é uma partição $\delta$-fina de $[a, c]$ e $D^{R}=$ $\left\{\alpha_{m-1}^{R}=c, \tau_{m}^{R}=c, \alpha_{m}, \ldots, \alpha_{k-1}, \tau_{k}, \alpha_{k}\right\}$ é uma partição $\delta$-fina de $[c, b]$.

Devido à escolha do calibre $\delta, D^{L}$ também é uma partição $\delta_{L}$-fina de $[a, c]$ e $D^{R}$ é uma partição $\delta_{R}$-fina de $[c, b]$. Portanto, para uma partição $\delta$-fina $D$ de $[a, b]$, temos a seguinte relação

$$
\begin{gathered}
\left\|S(U, D)-I_{L}-I_{R}\right\|=\left\|S\left(U, D^{L}\right)+S\left(U, D^{R}\right)-I_{L}-I_{R}\right\| \leq \\
\leq\left\|S\left(U, D_{L}\right)-I_{L}\right\|+\left\|S\left(U, D_{R}\right)-I_{R}\right\|<2 \varepsilon .
\end{gathered}
$$

Pela definição, isto implica a existência da integral $\int_{a}^{b} D U(\tau, t)$ e também a seguinte igualdade

$$
\int_{a}^{b} D U(\tau, t)=\int_{a}^{c} D U(\tau, t)+\int_{c}^{b} D U(\tau, t) .
$$

Portanto, temos o resultado desejado.

Pelos teoremas acima, percebemos que muitas propriedades das integrais de Lebesgue e de Riemann também valem para a integral de Kurzweil.

O resultado que enunciaremos abaixo é conhecido como Lema de Saks-Henstock e é devido ao polonês Stanislaw Saks e ao inglês Ralph Henstock, ambos matemáticos. O Lema de Saks-Henstock diz que a integral em "partes" de $[a, b]$ corresponde à soma de Riemann relativamente à estas "partes" de $[a, b]$ para os mesmos valores de $\varepsilon$ e $\delta$ da definição de integral sobre todo intervalo $[a, b]$.

Lema 1.8 (Saks-Henstock - [26], Lema 1.13). Seja $U:[a, b] \times[a, b] \rightarrow X$ integrável sobre 
$[a, b]$. Dado $\varepsilon>0$, seja $\delta$ uma função calibre em $[a, b]$ tal que

$$
\left\|\sum_{j=1}^{k}\left[U\left(\tau_{j}, \alpha_{j}\right)-U\left(\tau_{j}, \alpha_{j-1}\right)\right]-\int_{a}^{b} D U(\tau, t)\right\|<\varepsilon
$$

para toda partição $\delta$-fina $D=\left\{\left(\tau_{j},\left[\alpha_{j-1}, \alpha_{j}\right]\right), j=1,2, \ldots, k\right\}$ de $[a, b]$. Se

$$
a \leq \beta_{1} \leq \xi_{1} \leq \gamma_{1} \leq \beta_{2} \leq \xi_{2} \leq \gamma_{2} \leq \ldots \leq \beta_{m} \leq \xi_{m} \leq \gamma_{m} \leq b
$$

representar um sistema $\delta$-fino $\left\{\left(\xi_{j},\left[\beta_{j}, \gamma_{j}\right]\right), j=1,2, \ldots, m\right\}$, isto é,

$$
\xi_{j} \in\left[\beta_{j}, \gamma_{j}\right] \subset\left(\xi_{j}-\delta\left(\xi_{j}\right), \xi_{j}+\delta\left(\xi_{j}\right)\right), \quad j=1,2, \ldots, m
$$

então teremos

$$
\left\|\sum_{j=1}^{m}\left[U\left(\xi_{j}, \beta_{j}\right)-U\left(\xi_{j}, \gamma_{j}\right)-\int_{\beta_{j}}^{\gamma_{j}} D U(\tau, t)\right]\right\|<\varepsilon .
$$

Prova: Por hipótese, $\beta_{j} \leq \gamma_{j}$ para $j=1,2, \ldots, m$. Mas podemos supor, sem perda de generalidade, que $\beta_{j}<\gamma_{j}$ para todo $j=1,2, \ldots, m$.

Denotemos $\gamma_{0}=a$ e $\beta_{m+1}=b$.

Se $\gamma_{j}<\beta_{j+1}$ para algum $j=0,1, \ldots, m$, então a existência da integral $\int_{a}^{b} D U(\tau, t)$ junto com o Teorema 1.6 vão implicar a existência da integral $\int_{\gamma_{j}}^{\beta_{j+1}} D U(\tau, t)$. Deste fato segue que, dado $\eta>0$, existe um calibre $\delta_{j}$ em $\left[\gamma_{j}, \beta_{j+1}\right]$ tal que $\delta_{j}(\tau)<\delta(\tau)$ para $\tau \in\left[\gamma_{j}, \beta_{j+1}\right]$ e para toda partição $\delta_{j}$-fina $D^{j}$ de $\left[\gamma_{j}, \beta_{j+1}\right]$, temos a desigualdade

$$
\left\|S\left(U, D^{j}\right)-\int_{\gamma_{j}}^{\beta_{j+1}} D U(\tau, t)\right\|<\frac{\eta}{m+1} .
$$

Se $\gamma_{j}=\beta_{j+1}$, então, pela relação acima, teremos $S\left(U, D^{j}\right)=0$.

Como $\bigcup_{j=0}^{m} D^{j} \cup\left\{\left(\xi_{j},\left[\beta_{j}, \gamma_{j}\right]\right), j=1,2, \ldots, m\right\}$ é uma partição $\delta$-fina de $[a, b]$ e a expressão

$$
\sum_{j=1}^{m}\left[U\left(\xi_{j}, \gamma_{j}\right)-U\left(\xi_{j}, \beta_{j}\right)\right]+\sum_{j=1}^{m} S\left(U, D^{j}\right)
$$

representa sua soma de Riemann, então, por (1.3), vale

$$
\left\|\sum_{j=1}^{m}\left[U\left(\xi_{j}, \gamma_{j}\right)-U\left(\xi_{j}, \beta_{j}\right)\right]+\sum_{j=1}^{m} S\left(U, D^{j}\right)-\int_{a}^{b} D U(\tau, t)\right\|<\varepsilon .
$$




$$
\begin{aligned}
& \text { Observe que vale } \int_{a}^{b} D U(\tau, t)=\sum_{j=1}^{m} \int_{\beta_{j}}^{\gamma_{j}} D U(\tau, t)+\sum_{j=0}^{m} \int_{\gamma_{j}}^{\beta_{j+1}} D U(\tau, t) \text {. Portanto, } \\
& \qquad \sum_{j=1}^{m}\left[U\left(\xi_{j}, \beta_{j}\right)-U\left(\xi_{j}, \gamma_{j}\right)-\int_{\beta_{j}}^{\gamma_{j}} D U(\tau, t)\right] \| \\
& =\left\|\sum_{j=1}^{m}\left[U\left(\xi_{j}, \beta_{j}\right)-U\left(\xi_{j}, \gamma_{j}\right)\right]+\sum_{j=0}^{m} \int_{\gamma_{j}}^{\beta_{j+1}} D U(\tau, t)-\int_{a}^{b} D U(\tau, t)\right\| \\
& \leq\left\|\sum_{j=1}^{m}\left[U\left(\xi_{j}, \beta_{j}\right)-U\left(\xi_{j}, \gamma_{j}\right)\right]-\int_{a}^{b} D U(\tau, t)+\sum_{j=0}^{m} S\left(U, D^{j}\right)\right\| \\
& \leq\left\|\sum_{j=1}^{m}\left[U\left(\xi_{j}, \beta_{j}\right)-U\left(\xi_{j}, \gamma_{j}\right)\right]-\int_{a}^{b} D U(\tau, t)+\sum_{j=0}^{m} S\left(U, D^{j}\right)\right\| \\
& +\sum_{j=0}^{m}\left\|\sum_{j=0}^{m} S\left(U, D^{j}\right)-\sum_{j=0}^{\beta_{j+1}} \int_{\gamma_{j}}^{\beta_{j+1}} D U(\tau, t)\right\| \\
&
\end{aligned}
$$

e, como esta desigualdade acontece para todo $\eta>0$, então a desigualdade (1.4) está satisfeita e o lema está provado.

O próximo teorema trata da extensão de Cauchy para a integral de Kurzweil.

Grosseiramente falando, a extensão de Cauchy de uma integral trata de estendê-la por integrais que conhecemos como "impróprias" nos casos da integral de Riemann e da integral de Lebesgue. No caso da integral de Kurzweil, as extensões de Cauchy também são integrais de Kurzweil e, portanto, não há "integrais impróprias", pois as integrais de Kurzweil contêm suas extensões de Cauchy. E é isto que o teorema seguinte nos diz.

Teorema 1.9 (Extensão de Cauchy - [26], Teorema 1.14). Se $U:[a, b] \times[a, b] \rightarrow X$ for uma função tal que para todo $c \in[a, b), U$ for integrável em $[a, c]$ e o limite

$$
\lim _{c \rightarrow b-}\left[\int_{a}^{c} D U(\tau, t)-U(b, c)+U(b, b)\right]=I \in X
$$

existir, então a função U será integrável em $[a, b]$ e teremos

$$
\int_{a}^{b} D U(\tau, t)=I
$$


Similarmente, se a função $U$ for integrável em $[c, b]$ para todo $c \in(a, b]$ e o limite

$$
\lim _{c \rightarrow a+}\left[\int_{c}^{b} D U(\tau, t)+U(a, c)-U(a, a)\right]=I \in X
$$

existir, então a função U será integrável sobre $[a, b]$ e teremos

$$
\int_{a}^{b} D U(\tau, t)=I
$$

Prova: Demonstraremos apenas a primeira parte deste teorema, já que a segunda parte segue analogamente.

Suponhamos que $\varepsilon>0$ seja dado. Pela relação

$$
\lim _{c \rightarrow b-}\left[\int_{a}^{c} D U(\tau, t)-U(b, c)+U(b, b)\right]=I \in X
$$

podemos encontrar um $B \in[a, b)$ tal que, para todo $c \in[B, b)$, vale a desigualdade

$$
\left\|\int_{a}^{c} D U(\tau, t)-U(b, c)+U(b, b)-I\right\|<\varepsilon .
$$

Suponhamos que $a=c_{0}<c_{1}<\ldots$ seja uma sequência crescente $\left(c_{p}\right)_{p=1}^{\infty}$ de pontos $c_{p} \in[a, b)$ tal que $\lim _{p \rightarrow \infty} c_{p}=b$. Por hipótese, $U$ é integrável em $\left[a, c_{p}\right]$ para todo $p=1,2, \ldots$ e, portanto, para todo $p=1,2, \ldots$, existe um calibre $\delta_{p}:\left[a, c_{p}\right] \rightarrow(0,+\infty)$ tal que para toda partição $\delta_{p}$-fina $D^{p}$ de $\left[a, c_{p}\right]$, temos

$$
\left\|S\left(U, D^{p}\right)-\int_{a}^{c_{p}} D U(\tau, t)\right\|<\frac{\varepsilon}{2^{p+1}}, \quad p=1,2, \ldots
$$

Por outro lado, para todo $\tau \in[a, b)$, existe exatamente um $p(\tau)=1,2, \ldots$ para o qual $\tau \in\left[c_{p(\tau)-1}, c_{p(\tau)}\right)$. Então dado $\tau \in[a, b)$, vamos escolher $\bar{\delta}(\tau)>0$ tal que $\bar{\delta}(\tau) \leq \delta_{p(\tau)}(\tau)$ $\mathrm{e}(\tau-\bar{\delta}(\tau), \tau+\bar{\delta}) \cap[a, b) \subset\left[a, c_{p(\tau)}\right)$.

Suponhamos que $c \in[a, b)$ seja dado e que

$$
\bar{D}=\left\{\alpha_{0}, \tau_{1}, \alpha_{1}, \ldots, \alpha_{k-2}, \tau_{k-1}, \alpha_{k-1}\right\}
$$

seja uma partição $\bar{\delta}$-fina de $[a, c]$. Se $p\left(\tau_{j}\right)=p$, então $\left[\alpha_{j-1}, \alpha_{j}\right] \subset\left(\tau_{j}-\bar{\delta}\left(\tau_{j}\right), \tau_{j}+\bar{\delta}\left(\tau_{j}\right)\right) \subset$ $\left[a, c_{p}\right]$ e também $\left[\alpha_{j-1}, \alpha_{j}\right] \subset\left(\tau_{j}-\delta_{p}\left(\tau_{j}\right), \tau_{j}+\delta_{p}\left(\tau_{j}\right)\right)$. 
Seja

$$
\sum_{j=1, p\left(\tau_{j}\right)=p}^{k-1}\left[U\left(\tau_{j}, \alpha_{j}\right)-U\left(\tau_{j}, \alpha_{j-1}\right)-\int_{\alpha_{j-1}}^{\alpha_{j}} D U(\tau, t)\right]
$$

a soma dos termos para a qual as marcas $\tau_{j}$ satisfazem a relação $\tau_{j} \in\left[c_{p-1}, c_{p}\right)$ na soma correspondente "total"

$$
\sum_{j=1}^{k-1}\left[U\left(\tau_{j}, \alpha_{j}\right)-U\left(\tau_{j}, \alpha_{j-1}\right)-\int_{\alpha_{j-1}}^{\alpha_{j}} D U(\tau, t)\right]
$$

Pela relação (1.6) e pelo Lema de Saks-Henstock (Lema 1.8), obtemos

$$
\left\|\sum_{j=1, p\left(\tau_{j}\right)=p}^{k-1}\left[U\left(\tau_{j}, \alpha_{j}\right)-U\left(\tau_{j}, \alpha_{j-1}\right)-\int_{\alpha_{j-1}}^{\alpha_{j}} D U(\tau, t)\right]\right\|<\frac{\varepsilon}{2^{p+1}}
$$

Assim

$$
\begin{gathered}
\left\|\sum_{j=1}^{k-1}\left[U\left(\tau_{j}, \alpha_{j}\right)-U\left(\tau_{j}, \alpha_{j-1}\right)\right]-\int_{a}^{c} D U(\tau, t)\right\|= \\
=\left\|\sum_{j=1}^{k-1}\left[U\left(\tau_{j}, \alpha_{j}\right)-U\left(\tau_{j}, \alpha_{j-1}\right)-\int_{\alpha_{j-1}}^{\alpha_{j}} D U(\tau, t)\right]\right\| \leq \\
\leq \sum_{p=1}^{\infty}\left\|\sum_{j=1, p\left(\tau_{j}\right)=p}^{k-1}\left[U\left(\tau, \alpha_{j}\right)-U\left(\tau_{j}, \alpha_{j-1}\right)-\int_{\alpha_{j-1}}^{\alpha_{j}} D U(\tau, t)\right]\right\| \leq \sum_{p=1}^{\infty} \frac{\varepsilon}{2^{p+1}}=\varepsilon .
\end{gathered}
$$

Agora, definiremos um calibre $\delta$ no intervalo $[a, b]$ da maneira seguinte. Para $\tau \in$ $[a, b)$, tomemos $0<\delta(\tau)<\min \{b-\tau, \bar{\delta}(\tau)\}$ tal que $0<\delta(b)<b-B$. Então, se $D=$ $\left\{\alpha_{0}, \tau_{1}, \alpha_{1}, \ldots, \alpha_{k-1}, \tau_{k}, \alpha_{k}\right\}$ for uma partição $\delta$-fina de $[a, b]$, pela escolha do calibre $\delta$, teremos $\tau_{k}=\alpha_{k}=b$ e $\alpha_{k-1} \in(B, b)$. Usando a desigualdade

$$
\left\|\int_{a}^{c} D U(\tau, t)-U(b, c)+U(b, b)-I\right\|<\varepsilon,
$$

obtemos a seguinte relação

$$
\begin{aligned}
\|S(U, D)-I\| & =\left\|\sum_{j=1}^{k-1}\left[U\left(\tau_{j}, \alpha_{j}\right)-U\left(\tau_{j}, \alpha_{j-1}\right)\right]+U\left(\tau_{k}, \alpha_{k}\right)-U\left(\tau_{k}, \alpha_{k-1}\right)-I\right\| \leq \\
& \leq\left\|\sum_{j=1}^{k-1}\left[U\left(\tau_{j}, \alpha_{j}\right)-U\left(\tau_{j}, \alpha_{j-1}\right)\right]-\int_{a}^{\alpha_{k-1}} D U(\tau, t)\right\|+
\end{aligned}
$$




$$
\begin{gathered}
+\left\|\int_{a}^{\alpha_{k-1}} D U(\tau, t)-U\left(b, \alpha_{k-1}\right)+U(b, b)-I\right\|< \\
<\varepsilon+\left\|\sum_{j=1}^{k-1}\left[U\left(\tau_{j}, \alpha_{j}\right)-U\left(\tau_{j}, \alpha_{j-1}\right)\right]-\int_{a}^{\alpha_{k-1}} D U(\tau, t)\right\| .
\end{gathered}
$$

Como $\alpha_{k-1}<b$ e $\bar{D}=\left\{\alpha_{0}, \tau_{1}, \alpha_{1}, \ldots, \alpha_{k-2}, \tau_{k-1}, \alpha_{k-1}\right\}$ é uma partição $\bar{\delta}$-fina de $\left[a, \alpha_{k-1}\right]$, o segundo termo do lado direito da última desigualdade pode ser estimado por $\varepsilon$, como foi mostrado anteriormente. Desta forma, obtemos

$$
\|S(U, D)-I\|<2 \varepsilon
$$

e esta desigualdade implica a existência da integral $\int_{a}^{b} D U(\tau, t)$ bem como a igualdade $\int_{a}^{b} D U(\tau, t)=I$.

Para a prova da outra parte do teorema, basta usarmos um raciocínio análogo.

Se $U:[a, b] \times[a, b] \rightarrow X$ for integrável, não é verdade que sua integral indefinida $\int_{a}^{s} D U(\tau, t), s \in[a, b]$, será sempre contínua. O teorema a seguir descreve como será esta integral indefinida.

Teorema 1.10 ([26], Teorema 1.16). Sejam $U:[a, b] \times[a, b] \rightarrow X$ uma função integrável em $[a, b]$ e $c \in[a, b]$. Então

$$
\lim _{s \rightarrow c}\left[\int_{a}^{s} D U(\tau, t)-U(c, s)+U(c, c)\right]=\int_{a}^{c} D U(\tau, t) .
$$

Prova: Seja $\varepsilon>0$ dado. Como $U$ é integrável em $[a, b]$, então existe uma função calibre $\delta$ em $[a, b]$ tal que a desigualdade

$$
\|S(U, D)-I\|=\left\|\sum_{j=1}^{k}\left[U\left(\tau_{j}, \alpha_{j}\right)-U\left(\tau_{j}, \alpha_{j-1}\right)\right]-I\right\|<\epsilon
$$

vale para toda partição $\delta$-fina $D=\left\{\left(\tau_{j},\left[\alpha_{j-1}, \alpha_{j}\right]\right), j=1,2, \ldots, k\right\}$ de $[a, b]$.

Seja $c \in[a, b]$ arbitrário. Se $s \in(c-\delta(c), c+\delta(c)) \cap[a, b]$, então, pelo Lema de Saks-Henstock (Lema 1.8), teremos

$$
\left\|U(c, s)-U(c, c)-\int_{c}^{s} D U(\tau, t)\right\|<\epsilon
$$

Portanto,

$$
\left\|\int_{a}^{s} D U(\tau, t)-U(c, s)+U(c, c)-\int_{a}^{c} D U(\tau, t)\right\|
$$




$$
=\left\|\int_{c}^{s} D U(\tau, t)-U(c, s)+U(c, c)\right\|<\epsilon,
$$

donde segue o resultado.

Vale ressaltar que, nas condições enunciadas acima, também vale o seguinte resultado análogo

$$
\lim _{s \rightarrow c}\left[\int_{s}^{b} D U(\tau, t)-U(c, s)+U(c, c)\right]=\int_{c}^{b} D U(\tau, t),
$$

cuja demonstração também é similar à demonstração anterior.

Para a integral de Kurzweil, também temos um teorema de mudança de variável que enunciaremos abaixo. Este teorema será de grande importância em nosso trabalho. Um caso particular deste teorema pode ser encontrado em [26], Teorema 1.18, para funções a valores em $\mathbb{R}^{n}$.

Teorema 1.11 (Mudança de variável - [26], Teorema 1.18). Suponhamos que $-\infty<c<$ $d<+\infty$ e que $\varphi:[c, d] \rightarrow \mathbb{R}$ seja uma função continua estritamente monótona em $[c, d]$. Seja $U:[\varphi(c), \varphi(d)] \times[\varphi(c), \varphi(d)] \rightarrow X$ uma função dada. Se uma das integrais

$$
\int_{\varphi(c)}^{\varphi(d)} D U(\tau, t), \quad \int_{c}^{d} D U(\varphi(\sigma), \varphi(s))
$$

existir, então a outra também existirá e teremos a igualdade

$$
\int_{\varphi(c)}^{\varphi(d)} D U(\tau, t)=\int_{c}^{d} D U(\varphi(\sigma), \varphi(s)) .
$$

Omitiremos a demonstração do teorema acima, pois ela é muito extensa e é completamente análoga à demonstração do Teorema 1.18 encontrado em [26].

Vale observar que o teorema acima também será válido quando a função $\varphi$ for apenas contínua e monótona, ou seja, não-decrescente ou não-crescente, não havendo necessidade de ser estritamente monótona.

Assim como podemos integrar por partes a integral de Riemman, também podemos integrar por partes a integral de Kurzweil, sendo esta mais uma ferramenta desta integral. A seguir, apresentamos um resultado a este respeito, que é uma generalização para funções a valores em espaço de Banach do Teorema 1.21 encontrado em [26].

Teorema 1.12 (Integração por partes - [26], Teorema 1.21). Seja $U:[a, b] \times[a, b] \rightarrow X$ uma função dada. Consideremos $U^{*}(\tau, t)=U(t, \tau)$ e $V(t, \tau)=U(\tau, \tau)-U(\tau, t)-U(t, \tau)+$ $U(t, t)$. Se duas das integrais $\int_{a}^{b} D U(\tau, t), \int_{a}^{b} D U^{*}(\tau, t)$ e $\int_{a}^{b} D V(\tau, t)$ existirem, então a 
terceira também existirá e teremos a igualdade

$$
\int_{a}^{b} D U(\tau, t)+\int_{a}^{b} D U^{*}(\tau, t)=U(b, b)-U(a, a)-\int_{a}^{b} D V(\tau, t) .
$$

Omitiremos, também, a demonstração deste teorema, pois ela é muito extensa e é completamente análoga a demonstração do Teorema 1.21 encontrado em [26].

O teorema abaixo é uma generalização do Teorema 1.35 encontrado em [26]. Incluiremos sua demonstração aqui.

Teorema 1.13 ([26], Teorema 1.35). Seja $U:[a, b] \times[a, b] \rightarrow X$ uma função integrável. $S e V:[a, b] \times[a, b] \rightarrow \mathbb{R}$ for integrável e se existir uma função calibre $\theta$ em $[a, b]$ tal que

$$
|t-\tau|\|U(\tau, t)-U(\tau, \tau)\| \leq(t-\tau)[V(\tau, t)-V(\tau, \tau)]
$$

para todo $t \in(\tau-\theta(\tau), \tau+\theta(\tau))$, então teremos a desigualdade

$$
\left\|\int_{a}^{b} D U(\tau, t)\right\| \leq \int_{a}^{b} D V(\tau, t) .
$$

Prova: Seja $\varepsilon>0$ dado. Como as integrais

$$
\int_{a}^{b} D U(\tau, t) \text { e } \int_{a}^{b} D V(\tau, t)
$$

existem, existe uma função calibre $\delta$ em $[a, b] \operatorname{com} \delta(s) \leq \theta(s)$ para $s \in[a, b]$ tal que para toda partição $\delta$-fina $D=\left\{\left(\tau_{j},\left[\alpha_{j-1}, \alpha_{j}\right]\right), j=1,2, \ldots, k\right\}$ de $[a, b]$, valem as desigualdades

$$
\left\|\sum_{j=1}^{k}\left[U\left(\tau_{j}, \alpha_{j}\right)-U\left(\tau_{j}, \alpha_{j-1}\right)\right]-\int_{a}^{b} D U(\tau, t)\right\|<\varepsilon
$$

$\mathrm{e}$

$$
\left|\sum_{j=1}^{k}\left[V\left(\tau_{j}, \alpha_{j}\right)-V\left(\tau_{j}, \alpha_{j-1}\right)\right]-\int_{a}^{b} D V(\tau, t)\right|<\varepsilon
$$

Por hipótese, para cada $i=1,2, \ldots, k$, temos

$$
\left(\alpha_{i}-\tau_{i}\right)\left\|U\left(\tau_{i}, \alpha_{i}\right)-U\left(\tau_{i}, \tau_{i}\right)\right\| \leq\left(\alpha_{i}-\tau_{i}\right)\left[V\left(\tau_{i}, \alpha_{i}\right)-V\left(\tau_{i}, \tau_{i}\right)\right]
$$

já que $\alpha_{i} \geq \tau_{i}$. Portanto,

$$
\left\|U\left(\tau_{i}, \alpha_{i}\right)-U\left(\tau_{i}, \tau_{i}\right)\right\| \leq V\left(\tau_{i}, \alpha_{i}\right)-V\left(\tau_{i}, \tau_{i}\right), \quad i=1,2, \ldots, k .
$$


Analogamente,

$$
\left\|U\left(\tau_{i}, \alpha_{i-1}\right)-U\left(\tau_{i}, \tau_{i}\right)\right\| \leq V\left(\tau_{i}, \tau_{i}\right)-V\left(\tau_{i}, \alpha_{i-1}\right), \quad i=1,2, \ldots, k .
$$

Desta forma, para cada $i=1,2, \ldots, k$, temos

$$
\begin{gathered}
\left\|U\left(\tau_{i}, \alpha_{i}\right)-U\left(\tau_{i}, \alpha_{i-1}\right)\right\| \leq\left\|U\left(\tau_{i}, \alpha_{i}\right)-U\left(\tau_{i}, \tau_{i}\right)\right\|+\left\|U\left(\tau_{i}, \tau_{i}\right)-U\left(\tau_{i}, \alpha_{i-1}\right)\right\| \\
\leq V\left(\tau_{i}, \alpha_{i}\right)-V\left(\tau_{i}, \tau_{i}\right)+V\left(\tau_{i}, \tau_{i}\right)-V\left(\tau_{i}, \alpha_{i-1}\right)=V\left(\tau_{i}, \alpha_{i}\right)-V\left(\tau_{i}, \alpha_{i-1}\right) .
\end{gathered}
$$

Por (1.8) e (1.9), obtemos

$$
\begin{gathered}
\left\|\int_{a}^{b} D U(\tau, t)\right\| \leq \\
\leq\left\|\sum_{j=1}^{k}\left[U\left(\tau_{j}, \alpha_{j}\right)-U\left(\tau_{j}, \alpha_{j-1}\right)\right]-\int_{a}^{b} D U(\tau, t)\right\|+\left\|\sum_{j=1}^{k}\left[U\left(\tau_{j}, \alpha_{j}\right)-U\left(\tau_{j}, \alpha_{j-1}\right)\right]\right\|< \\
<\varepsilon+\sum_{j=1}^{k}\left[V\left(\tau_{j}, \alpha_{j}\right)-V\left(\tau_{j}, \alpha_{j-1}\right)\right]-\int_{a}^{b} D V(\tau, t)+\int_{a}^{b} D V(\tau, t)< \\
<2 \varepsilon+\int_{a}^{b} D V(\tau, t) .
\end{gathered}
$$

Daí, como $\varepsilon$ é arbitrário, temos o resultado desejado.

\subsection{Noções básicas de EDOGs}

Dado um $c>0$, seja $O=B_{c}=\{x \in X ;\|x\|<c\}$. Seja, também, $(a, b) \subset \mathbb{R}$ um intervalo aberto com $-\infty<a<b<\infty$. Vamos considerar o conjunto $\Omega=O \times(a, b)$.

Seja $G: \Omega \rightarrow X$ uma função a valores em $X$ definida para $(x, t) \in \Omega$, onde $x \in X$ e $t \in \mathbb{R}$. Lembramos o leitor que estamos considerando $X$ como sendo um espaço de Banach.

Definição 1.14. Uma função $x:[\alpha, \beta] \rightarrow X$ será dita uma solução da equação diferencial ordinária generalizada

$$
\frac{d x}{d \tau}=D G(x, t)
$$

no intervalo $[\alpha, \beta] \subset \mathbb{R}$, se $(x(t), t) \in \Omega$ para todo $t \in[\alpha, \beta]$ e se a igualdade

$$
x\left(s_{2}\right)-x\left(s_{1}\right)=\int_{s_{1}}^{s_{2}} D G(x(\tau), t)
$$


acontecer para todo $s_{1}, s_{2} \in[\alpha, \beta]$.

A integral do lado direito da igualdade (1.11) deve ser entendida como sendo a integral de Kurzweil definida anteriormente.

É importante perceber que a notação mencionada em (1.10) é apenas simbólica. A letra $D$ indica que (1.10) é uma equação diferencial generalizada, definida acima. É importante ressaltar que mesmo usando o símbolo $\frac{d x}{d \tau}$ na igualdade acima, não significa necessariamente que a solução tenha uma derivada.

Alternativamente, podemos usar a seguinte definição para um problema de valor inicial no sentido generalizado.

Definição 1.15. Uma função $x:[\alpha, \beta] \rightarrow X$ será uma solução da equação diferencial generalizada (1.10) com condição inicial $x\left(t_{0}\right)=z_{0}$ no intervalo $[\alpha, \beta] \subset \mathbb{R}$, se $t_{0} \in[\alpha, \beta]$, $(x(t), t) \in \Omega$ para todo $t \in[\alpha, \beta]$ e se a igualdade

$$
x(v)-z_{0}=\int_{t_{0}}^{v} D G(x(\tau), t)
$$

acontecer para todo $v \in[\alpha, \beta]$.

Proposição 1.16 ([26], Proposição 3.5). Se uma função $x:[\alpha, \beta] \rightarrow X$ for uma solução da equação diferencial ordinária generalizada (1.10) em $[\alpha, \beta]$, então para todo $\gamma \in[\alpha, \beta]$ fixado, a igualdade

$$
x(s)=x(\gamma)+\int_{\gamma}^{s} D G(x(\tau), t), \quad s \in[\alpha, \beta]
$$

estará satisfeita. Se uma função $x:[\alpha, \beta] \rightarrow X$ satisfizer a equação integral (1.12) para algum $\gamma \in[\alpha, \beta]$, então $x$ será uma solução da equação diferencial ordinária generalizada (1.10).

Prova: A primeira parte do teorema segue diretamente da definição de solução de uma equação diferencial generalizada dada acima, tomando-se $s_{1}=\gamma$ e $s_{2}=s$.

Por outro lado, se $x:[\alpha, \beta] \rightarrow X$ satisfizer a equação (1.12), então para $s=s_{2}$, teremos a relação abaixo

$$
x\left(s_{2}\right)=x(\gamma)+\int_{\gamma}^{s_{2}} D G(x(\tau), t), \quad \gamma \in[\alpha, \beta] .
$$

Tomando $s=s_{1}$ em (1.12), teremos

$$
x\left(s_{1}\right)=x(\gamma)+\int_{\gamma}^{s_{1}} D G(x(\tau), t) \quad \gamma \in[\alpha, \beta] .
$$


Daí, pelas relações acima e pela aditividade da integral, obtemos

$$
x\left(s_{2}\right)-x\left(s_{1}\right)=\int_{s_{1}}^{s_{2}} D G(x(\tau), t)
$$

para todo $s_{1}, s_{2} \in[\alpha, \beta]$. Logo $x$ é uma solução de (1.10).

A proposição seguinte descreve as soluções de (1.10) em função de $G$. Em particular, se $G$ for contínua na segunda variável, então a solução $x$ de (1.10) será uma função contínua. Proposição 1.17 ([26], Proposição 3.6). Se $x:[\alpha, \beta] \rightarrow X$ for uma solução da equação diferencial generalizada (1.10) em $[\alpha, \beta]$, então

$$
\lim _{s \rightarrow \sigma}[x(s)-G(x(\sigma), s)+G(x(\sigma), \sigma)]=x(\sigma)
$$

para todo $\sigma \in[\alpha, \beta]$.

Prova: Seja $\sigma \in[\alpha, \beta]$ fixo. Como $x$ é solução da equação (1.10), então, pela Proposição 1.16, temos a relação abaixo

$$
x(s)=x(\sigma)+\int_{\sigma}^{s} D G(x(\tau), t), \quad s \in[\alpha, \beta]
$$

Logo,

$$
x(s)-G(x(\sigma), s)+G(x(\sigma), \sigma)=\int_{\sigma}^{s} D G(x(\tau), t)-G(x(\sigma), s)+G(x(\sigma), \sigma)+x(\sigma),
$$

para todo $s \in[\alpha, \beta]$. Daí, pelo Teorema 1.10, temos

$$
\lim _{s \rightarrow \sigma}\left[\int_{\sigma}^{s} D G(x(\tau), t)-G(x(\sigma), s)+G(x(\sigma), \sigma)\right]=\int_{\sigma}^{\sigma} D G(x(\tau), t)=0 .
$$

Assim, a relação acima e a igualdade (1.14) implicam a existência do limite $\lim _{s \rightarrow \sigma}[x(s)-$ $G(x(\sigma), s)+G(x(\sigma), \sigma)-x(\sigma)]$ e vale

$$
\begin{gathered}
\lim _{s \rightarrow \sigma}[x(s)-G(x(\sigma), s)+G(x(\sigma), \sigma)] \\
=\lim _{s \rightarrow \sigma}\left[\int_{\sigma}^{s} D G(x(\tau), t)-G(x(\sigma), s)+G(x(\sigma), \sigma)+x(\sigma)\right]=x(\sigma) .
\end{gathered}
$$

que é equivalente a (1.13) e a prova está completa.

A seguir, vamos introduzir uma classe especial de funções $G: \Omega \mapsto X$ para a qual é possível obter informações mais específicas sobre as soluções da equação diferencial 
generalizada (1.10). Iremos supor, ao longo deste trabalho, que $h:[a, b] \mapsto \mathbb{R}$ é uma função não decrescente definida em $[a, b]$.

Definição 1.18. Diremos que uma função $G: \Omega \rightarrow X$ pertence à classe $\mathcal{F}(\Omega, h)$, se

$$
\left\|G\left(x, t_{2}\right)-G\left(x, t_{1}\right)\right\| \leq\left|h\left(t_{2}\right)-h\left(t_{1}\right)\right|
$$

para todo $\left(x, t_{2}\right),\left(x, t_{1}\right) \in \Omega$ e se

$$
\left\|G\left(x, t_{2}\right)-G\left(x, t_{1}\right)-G\left(y, t_{2}\right)+G\left(y, t_{1}\right)\right\| \leq\|x-y\|\left|h\left(t_{2}\right)-h\left(t_{1}\right)\right|
$$

para todo $\left(x, t_{2}\right),\left(x, t_{1}\right),\left(y, t_{2}\right),\left(y, t_{1}\right) \in \Omega$.

O lema a seguir é uma generalização, para o caso das funções a valores em $X$, do Lema 3.9 encontrado em [26]. As demonstrações de tais resultados são similares.

Lema 1.19 ([26], Lema 3.9). Suponhamos que $G: \Omega \rightarrow X$ satisfaça a condição (1.15). $S e[\alpha, \beta] \subset[a, b]$ e $x:[\alpha, \beta] \rightarrow X$ for tal que $(x(t), t) \in \Omega$ para todo $t \in[\alpha, \beta]$ e se a integral $\int_{\alpha}^{\beta} D G(x(\tau), t)$ existir, então para quaisquer $s_{1}, s_{2} \in[\alpha, \beta]$, valerá a desigualdade

$$
\left\|\int_{s_{1}}^{s_{2}} D G(x(\tau), t)\right\| \leq\left|h\left(s_{2}\right)-h\left(s_{1}\right)\right| .
$$

Prova: Pela desigualdade (1.15) e pelo fato de $h$ ser não decrescente, temos a desigualdade abaixo

$$
|t-\tau||| G(x(\tau), t)-G(x(\tau), \tau) \| \leq|t-\tau||h(t)-h(\tau)|
$$

para quaisquer $t, \tau \in[\alpha, \beta]$.

Tomando-se $V(\tau, t)=h(t)$, a integral $\int_{\alpha}^{\beta} D V(\tau, t)=\int_{\alpha}^{\beta} d h(t)$ existe e

$$
\int_{s_{1}}^{s_{2}} d h(t)=h\left(s_{2}\right)-h\left(s_{1}\right)
$$

para quaisquer $s_{1}, s_{2} \in[\alpha, \beta]$. Como todas as hipóteses do Teorema 1.13 estão satisfeitas, podemos aplicá-lo para obter a relação abaixo

$$
\left\|\int_{s_{1}}^{s_{2}} D G(x(\tau), t)\right\| \leq\left|\int_{s_{1}}^{s_{2}} D V(\tau, t)\right|
$$

e, portanto,

$$
\left\|\int_{s_{1}}^{s_{2}} D G(x(\tau), t)\right\| \leq\left|h\left(s_{2}\right)-h\left(s_{1}\right)\right|
$$


para quaisquer $s_{1}, s_{2} \in[\alpha, \beta]$.

A seguir, vamos definir algumas classes de funções que utilizaremos ao longo deste trabalho.

Definição 1.20. Uma função $f:[a, b] \rightarrow \mathbb{R}$ será dita uma função escada finita, se existir uma divisão finita $a=\beta_{0}<\beta_{1}<\ldots<\beta_{m}=b$ tal que em cada intervalo aberto $\left(\beta_{i-1}, \beta_{i}\right), i=1,2, \ldots, m$, a função é identicamente igual a uma constante $c_{i} \in \mathbb{R}$.

Definição 1.21. Uma função $f:[a, b] \rightarrow \mathbb{R}$ será dita uma função regrada no intervalo $[a, b]$, se os limites laterais $f(s-)$ e $f(s+)$ existirem para todo $s \in(a, b]$ e $s \in[a, b)$ respectivamente.

É um fato conhecido que toda função regrada em $[a, b]$ é limitada neste intervalo e é o limite uniforme de funções escada finitas. É claro, também, que toda função de variação limitada em $[a, b]$ é regrada em $[a, b]$. Veja [16].

O lema a seguir vai implicar o fato de que as soluções de (1.10) são de variação limitada, contanto que $G$ satisfaça a condição (1.15).

Lema 1.22 ([26], Lema 3.10). Suponhamos que $G: \Omega \rightarrow X$ satisfaça a condição (1.15). $S e[\alpha, \beta] \subset[a, b]$ e $x:[\alpha, \beta] \rightarrow X$ for uma solução de (1.10), então valerá a desigualdade

$$
\left\|x\left(s_{1}\right)-x\left(s_{2}\right)\right\| \leq\left|h\left(s_{2}\right)-h\left(s_{1}\right)\right|
$$

para quaisquer $s_{1}, s_{2} \in[\alpha, \beta]$.

Prova: O resultado segue diretamente do Lema 1.19 e da definição de solução da equação diferencial generalizada (1.10).

Denotaremos a variação de uma função $x:[\alpha, \beta] \rightarrow X$ por $\operatorname{var}_{\alpha}^{\beta} x$ e o espaço das funções de variação limitada $x:[\alpha, \beta] \rightarrow X$ por $B V([\alpha, \beta], X)$ com a norma usual da variação dada por

$$
\|x\|_{B V}=\|x(\alpha)\|+\operatorname{var}_{\alpha}^{\beta} x
$$

Corolário 1.23 ([26], Corolário 3.11). Suponhamos que $G: \Omega \rightarrow X$ satisfaça a condição (1.15). Se $[\alpha, \beta] \subset(a, b)$ e $x:[\alpha, \beta] \rightarrow X$ for uma solução de (1.10), então $x$ será de variação limitada em $[\alpha, \beta]$ e

$$
\operatorname{var}_{\alpha}^{\beta} x \leq h(\beta)-h(\alpha)<+\infty
$$

Além disso, todo ponto em $[\alpha, \beta]$ no qual a função $h$ é contínua também é um ponto de continuidade da solução $x:[\alpha, \beta] \rightarrow X$. 
Prova: Seja $\alpha=s_{0}<s_{1}<\ldots<s_{k}=\beta$ uma divisão arbitrária do intervalo $[\alpha, \beta]$. Como todas as hipóteses do lema anterior estão satisfeitas, a desigualdade (1.18) implica que

$$
\sum_{j=1}^{k}\left\|x\left(s_{j}\right)-x\left(s_{j-1}\right)\right\| \leq \sum_{j=1}^{k}\left[h\left(s_{j}\right)-h\left(s_{j-1}\right)\right]=h(\beta)-h(\alpha) .
$$

Passando o supremo sobre todas as divisões de $[\alpha, \beta]$, temos a desigualdade desejada.

A segunda afirmação segue imediatamente da desigualdade (1.18).

O próximo lema nos diz que se $G$ satisfizer (1.15), então as descontinuidades de uma solução qualquer de (1.10) serão de primeira espécie, como era de se esperar já que pelo Corolário 1.23, as soluções são funções de variação limitada e, portanto, regradas. Em particular, o lema a seguir descreve como serão os "saltos" de $x$, quando existirem.

Lema 1.24 ([26], Lema 3.12). Se $x:[\alpha, \beta] \rightarrow X$ for uma solução de (1.10) e $G: \Omega \rightarrow X$ satisfizer a condição (1.15), então

$$
x(\sigma+)-x(\sigma)=\lim _{s \rightarrow \sigma+} x(s)-x(\sigma)=G(x(\sigma), \sigma+)-G(x(\sigma), \sigma)
$$

$\operatorname{para} \sigma \in[\alpha, \beta) e$

$$
x(\sigma)-x(\sigma-)=x(\sigma)-\lim _{s \rightarrow \sigma-} x(s)=G(x(\sigma), \sigma)-G(x(\sigma), \sigma-)
$$

para $\sigma \in(\alpha, \beta]$, onde

$$
G(x, \sigma+)=\lim _{s \rightarrow \sigma+} G(x, s), \quad \text { para } \sigma \in[\alpha, \beta)
$$

e

$$
G(x, \sigma-)=\lim _{s \rightarrow \sigma-} G(x, s), \quad \text { para } \sigma \in(\alpha, \beta] .
$$

Prova: Como a função $h$ é de variação limitada, então $h$ possui limites laterais em todos os pontos do intervalo $[\alpha, \beta]$. Disso, segue que os limites $G(x, \sigma+)$ e $G(x, \sigma-)$ existem. Este fato e a condição (1.15) garantem a existência dos limites laterais correspondentes da função $G(x, \sigma):[\alpha, \beta] \rightarrow X$ para todo $x \in B_{c}$.

Pela definição de uma solução de (1.10), temos a igualdade abaixo

$$
x(\sigma)-x(s)=\int_{s}^{\sigma} D G(x(\tau), t)
$$


para $\alpha \leq s<\sigma \leq \beta$. Pelo Teorema 1.10, temos

$$
\lim _{\sigma \rightarrow s^{+}} x(\sigma)-x(s)=\lim _{\sigma \rightarrow s^{+}} \int_{\sigma}^{s} D G(x(\tau), t)=\lim _{\sigma \rightarrow s^{+}}[G(x(s), \sigma)-G(x(s), s)]
$$

e a primeira relação do enunciado está satisfeita.

A segunda relação pode ser provada analogamente.

A demonstração do lema abaixo foi inspirada na demonstração do Corolário 3.15 encontrado em [26] e trata da existência da integral $\int_{\alpha}^{\beta} D G(x(\tau), t)$ quando $x$ for uma função escada finita. Consideraremos, entretanto, funções a valores em espaços de Banach.

Lema 1.25 ([26], Corolário 3.15). Se $G \in \mathcal{F}(\Omega, h)$ e $\varphi:[\alpha, \beta] \rightarrow X$ for uma função escada finita, então existirá a integral

$$
\int_{\alpha}^{\beta} D G(\varphi(\tau), t)
$$

Prova: Se $\varphi$ for uma função escada finita, então existirá uma divisão $\alpha=s_{0}<s_{1}<$ $\ldots<s_{k}=\beta$ de $[\alpha, \beta]$ tal que $\varphi(s)=c_{j} \in X$ para $s \in\left(s_{j-1}, s_{j}\right), j=1,2, \ldots, k$ onde $c_{j}, j=1,2, \ldots, k$ são constantes. Suponhamos que $(\varphi(s), s) \in \Omega$ para todo $s \in[\alpha, \beta]$. Pela definição da integral, é fácil ver que se $s_{j-1}<\sigma_{1}<\sigma_{2}<s_{j}$, então a integral $\int_{\sigma_{1}}^{\sigma_{2}} D G(\varphi(\tau), t)$ existirá e

$$
\int_{\sigma_{1}}^{\sigma_{2}} D G(\varphi(\tau), t)=G\left(c_{j}, \sigma_{2}\right)-G\left(c_{j}, \sigma_{1}\right)
$$

Suponhamos que $\sigma_{0} \in\left(s_{j-1}, s_{j}\right)$ seja dado. Então temos a seguinte a relação:

$$
\begin{aligned}
& \lim _{s \rightarrow s_{j-1}^{+}}\left[\int_{s}^{\sigma_{0}} D G(\varphi(\tau), t)+G\left(\varphi\left(s_{j-1}\right), s\right)-G\left(\varphi\left(s_{j-1}\right), s_{j-1}\right)\right]= \\
= & \lim _{s \rightarrow s_{j-1}^{+}}\left[G\left(c_{j}, \sigma_{0}\right)-G\left(c_{j}, s\right)+G\left(\varphi\left(s_{j-1}\right), s\right)-G\left(\varphi\left(s_{j-1}\right), s_{j-1}\right)\right]= \\
= & G\left(c_{j}, \sigma_{0}\right)-G\left(c_{j}, s_{j-1}+\right)+G\left(\varphi\left(s_{j-1}\right), s_{j-1}+\right)-G\left(\varphi\left(s_{j-1}\right), s_{j-1}\right) .
\end{aligned}
$$

Portanto, pelo Teorema 1.9, a integral $\int_{s_{j-1}}^{\sigma_{0}} D G(\varphi(\tau), t)$ existe e é igual ao limite calculado acima.

Similarmente, podemos mostrar que a integral $\int_{\sigma_{0}}^{s_{j}} D G(\varphi(\tau), t)$ também existe e vale

$$
\int_{\sigma_{0}}^{s_{j}} D G(\varphi(\tau), t)=G\left(c_{j}, s_{j}-\right)-G\left(c_{j}, \sigma_{0}\right)-G\left(\varphi\left(s_{j}\right), s_{j}-\right)+G\left(\varphi\left(s_{j}\right), s_{j}\right) .
$$


Portanto, pelo Teorema 1.7, obtemos a existência da integral $\int_{s_{j-1}}^{s_{j}} D G(\varphi(\tau), t)$ e

$$
\begin{gathered}
\int_{s_{j-1}}^{s_{j}} D G(\varphi(\tau), t)=G\left(c_{j}, s_{j}-\right)-G\left(c_{j}, \sigma_{0}\right)-G\left(\varphi\left(s_{j}\right), s_{j}-\right)+G\left(\varphi\left(s_{j}\right), s_{j}\right)+ \\
+G\left(c_{j}, \sigma_{0}\right)-G\left(c_{j}, s_{j-1}\right)+G\left(\varphi\left(s_{j-1}\right), s_{j-1}+\right)-G\left(\varphi\left(s_{j-1}\right), s_{j-1}\right)= \\
=G\left(c_{j}, s_{j}-\right)-G\left(c_{j}, s_{j-1}+\right)+G\left(\varphi\left(s_{j-1}\right), s_{j-1}+\right)- \\
-G\left(\varphi\left(s_{j-1}\right), s_{j-1}\right)-G\left(\varphi\left(s_{j}\right), s_{j}-\right)+G\left(\varphi\left(s_{j}\right), s_{j}\right) .
\end{gathered}
$$

Repetindo este argumento para todo intervalo $\left[s_{j-1}, s_{j}\right], j=1,2, \ldots, k$, e usando novamente o Teorema 1.7, obtemos a existência da integral $\int_{\alpha}^{\beta} D G(\varphi(\tau), t)$ assim como a igualdade

$$
\begin{gathered}
\int_{\alpha}^{\beta} D G(\varphi(\tau), t)=\sum_{j=1}^{k}\left[G\left(c_{j}, s_{j}-\right)-G\left(c_{j}, s_{j-1}+\right)\right]+ \\
+\sum_{j=1}^{k}\left[G\left(\varphi\left(s_{j-1}\right), s_{j-1}+\right)-G\left(\varphi\left(s_{j-1}\right), s_{j-1}\right)-G\left(\varphi\left(s_{j}\right), s_{j}-\right)+G\left(\varphi\left(s_{j}\right), s_{j}\right)\right] .
\end{gathered}
$$

Isto completa a prova.

Prosseguimos para provar a existência da integral $\int_{\alpha}^{\beta} D G(x(\tau), t)$ quando $x$ for uma função regrada.

Teorema 1.26. Suponhamos que a função $G \in \mathcal{F}(\Omega, h)$ seja dada e que $x:[\alpha, \beta] \rightarrow X$ seja o limite uniforme de uma sequência $\left(x_{k}\right)_{k \in \mathbb{N}}$ de funções escada finitas $x_{k}:[\alpha, \beta] \rightarrow X$ tais que $(x(s), s) \in \Omega e\left(x_{k}(s), s\right) \in \Omega$ para todo $k \in \mathbb{N}$ e todo $s \in[\alpha, \beta]$. Então a integral $\int_{\alpha}^{\beta} D G(x(\tau), t)$ existe e vale a igualdade

$$
\int_{\alpha}^{\beta} D G(x(\tau), t)=\lim _{k \rightarrow \infty} \int_{\alpha}^{\beta} D G\left(x_{k}(\tau), t\right)
$$

Prova: Para cada $k \in \mathbb{N}$, a integral $\int_{\alpha}^{\beta} D G\left(x_{k}(\tau), t\right)$ existe pelo lema anterior.

Dado $\varepsilon>0$, seja $k_{0} \in \mathbb{N}$ tal que para $k \geq k_{0}$, tenhamos

$$
\left\|x_{k}(s)-x(s)\right\|<\frac{\varepsilon}{[h(\beta)-h(\alpha)]}, \quad s \in[\alpha, \beta]
$$

e seja $\delta$ um calibre em $[a, b]$ tal que, para $k \geq k_{0}$, tenhamos

$$
\left\|\sum_{i=1}^{m}\left[G\left(x_{k}\left(\tau_{i}\right), t_{i}\right)-G\left(x_{k}\left(\tau_{i}\right), t_{i-1}\right)\right]-\int_{\alpha}^{\beta} D G\left(x_{k}(\tau), t\right)\right\|<\frac{\varepsilon}{2}
$$


para toda partição $\delta$-fina $D=\left\{\alpha_{0} \leq \tau_{1} \leq t_{1} \leq \ldots \leq t_{m-1} \leq \tau_{m} \leq \beta\right\}$ de $[\alpha, \beta]$. Então para todo $k \geq k_{0}$, vale

$$
\begin{gathered}
\left\|\sum_{i=1}^{m}\left[G\left(x\left(\tau_{i}\right), t_{i}\right)-G\left(x\left(\tau_{i}\right), t_{i-1}\right)\right]-\int_{\alpha}^{\beta} D G\left(x_{k}(\tau), t\right)\right\| \leq \\
\leq \sum_{i=1}^{m}\left\|G\left(x\left(\tau_{i}\right), t_{i}\right)-G\left(x\left(\tau_{i}\right), t_{i-1}\right)-G\left(x_{k}\left(\tau_{i}\right), t_{i}\right)+G\left(x_{k}\left(\tau_{i}\right), t_{i-1}\right)\right\|+ \\
+\left\|\sum_{i=1}^{m}\left[G\left(x_{k}\left(\tau_{i}\right), t_{i}\right)-G\left(x_{k}\left(\tau_{i}\right), t_{i-1}\right)\right]-\int_{\alpha}^{\beta} D G\left(x_{k}(\tau), t\right)\right\| \leq \\
\leq \sum_{i=1}^{m}\left[h\left(t_{i}\right)-h\left(t_{i-1}\right)\right] \max _{i}\left\|x\left(\tau_{i}\right)-x_{k}\left(\tau_{i}\right)\right\|+\frac{\varepsilon}{2}= \\
=[h(\beta)-h(\alpha)] \max _{i}\left\|x\left(\tau_{i}\right)-x_{k}\left(\tau_{i}\right)\right\|+\frac{\varepsilon}{2}<\varepsilon
\end{gathered}
$$

e a prova está completa.

A demonstração do Teorema 1.26 não é análoga à prova apresentada em [26], Teorema 3.14 , para funções a valores em $\mathbb{R}^{n}$, onde foi usado o conceito de equiintegrabilidade e um teorema de convergência para funções equiintegráveis. Aqui, usamos fortemente o fato da função ser o limite uniforme de funções escada finitas. Em [26], por outro lado, usou-se convergência pontual.

O corolário abaixo é uma generalização do Corolário 3.16 encontrado em [26].

Corolário 1.27 ([26], Corolário 3.16). Se $G \in \mathcal{F}(\Omega, h)$ for uma função dada e $x:[\alpha, \beta] \rightarrow$ $X,[\alpha, \beta] \subset(a, b)$, for uma função regrada (em particular, uma função de variação limitada) em $[\alpha, \beta]$ tal que $(x(s), s) \in \Omega$ para todo $s \in[\alpha, \beta]$, então existirá a integral

$$
\int_{\alpha}^{\beta} D G(x(\tau), t)
$$

Prova: Como toda função regrada é o limite uniforme de funções escada finitas, então todas as hipóteses do Teorema 1.26 estão satisfeitas e o resultado segue.

Chegamos ao final desta seção conhecendo certas hipóteses que $x$ e $G$ devem satisfazer para que a integral

$$
\int_{\alpha}^{\beta} D G(x(\tau), t)
$$

exista. Agora, portanto, estamos em condições de investigar propriedades básicas sobre as soluções de (1.10) que são descritas através desta integral. 


\subsection{Existência e unicidade de soluções}

Nesta seção, iremos supor que $\Omega=O \times(a, b)$, onde $(a, b) \subset \mathbb{R}, O=B_{c}=\{x \in$ $X ;\|x\|<c\}, \operatorname{com} c>0$ e $-\infty<a<b<+\infty, h:[a, b] \rightarrow \mathbb{R}$ é uma função não decrescente e contínua à esquerda.

Vamos considerar a equação diferencial generalizada

$$
\frac{d x}{d \tau}=D G(x, t)
$$

onde $G: \Omega \rightarrow X$ pertence à classe $\mathcal{F}(\Omega, h)$ descrita anteriormente. Nestas condições, uma solução de (1.20) é uma função de variação limitada (veja o Corolário 1.23) que, pelo Lema 1.22, também é contínua à esquerda, e pelo Lema 1.24 tem descontinuidades de primeira espécie. Dessa forma, se para algum $t_{0} \in(a, b)$, o valor da solução $x$ de $(1.20)$ for $x\left(t_{0}\right)=\widetilde{x}$, então o limite à direita no ponto $t_{0}$ satisfará

$$
x\left(t_{0}+\right)=x\left(t_{0}\right)+G\left(x\left(t_{0}\right), t_{0}+\right)-G\left(x\left(t_{0}\right), t_{0}\right)=\widetilde{x}+G\left(\widetilde{x}, t_{0}+\right)-G\left(\widetilde{x}, t_{0}\right) .
$$

Como estamos considerando a possibilidade de (1.20) admitir solução descontínua, pode acontecer o fato de que, para algum $\widetilde{x} \in B_{c}$, isto é, para algum $\left(\widetilde{x}, t_{0}\right) \in \Omega$, o valor

$$
\widetilde{x}+=\widetilde{x}+G\left(\widetilde{x}, t_{0}+\right)-G\left(\widetilde{x}, t_{0}\right)
$$

não pertença a $O$. Assim, para provar o teorema de existência local de uma solução de $(1.20)$, satisfazendo a condição inicial $x\left(t_{0}\right)=\widetilde{x}$, fazemos a seguinte hipótese natural

$$
\widetilde{x}+=\widetilde{x}+G\left(\widetilde{x}, t_{0}+\right)-G\left(\widetilde{x}, t_{0}\right) \in O .
$$

O teorema a seguir trata da existência local e unicidade de soluções de (1.20).

Teorema 1.28 ([7], Teorema 2.15). Seja $G: \Omega \rightarrow X$ uma função pertencente à classe $F(\Omega, h)$, onde $\Omega=O \times(a, b)$, e a função $h$ é contínua à esquerda e não decrescente em $[a, b]$. Então para todo $\left(\widetilde{x}, t_{0}\right) \in \Omega$ satisfazendo

$$
\widetilde{x}+=\widetilde{x}+G\left(\widetilde{x}, t_{0}-\right)-G\left(\widetilde{x}, t_{0}\right) \in O,
$$

teremos $\left(\widetilde{x}+, t_{0}\right) \in \Omega$ e existirá um $\Delta>0$ tal que, no intervalo $\left[t_{0}-\Delta, t_{0}+\Delta\right]$, existirá uma única solução $x:\left[t_{0}-\Delta, t_{0}+\Delta\right] \rightarrow X$ da equação diferencial generalizada (1.20) satisfazendo $x\left(t_{0}\right)=\widetilde{x}$. 
Prova: Primeiramente, suponhamos que $t_{0}$ seja um ponto de continuidade da função $h$. Como $\widetilde{x} \in O$, existe $\rho>0$ tal que $(\widetilde{x}-\rho, \widetilde{x}+\rho) \subset O$. Como $h$ é contínua em $t_{0}$, então dado $\varepsilon>0$, existe $\Delta>0$ tal que $\left|t-t_{0}\right|<\Delta$ implica $\left|h(t)-h\left(t_{0}\right)\right|<\varepsilon$. Portanto, se $x \in X$ for tal que $\|x-\widetilde{x}\| \leq\left|h(t)-h\left(t_{0}\right)\right|<\varepsilon$, então $x \in O$, para $\varepsilon<\rho$.

Suponhamos que $\Delta>0$ seja tal que $\left[t_{0}-\Delta, t_{0}+\Delta\right] \subset(a, b), h\left(t_{0}+\Delta\right)-h\left(t_{0}-\Delta\right)<\frac{1}{2}$ e $\|x-\tilde{x}\| \leq\left|h(t)-h\left(t_{0}\right)\right|$ implica que $x \in O$.

Seja $\mathcal{A}$ o conjunto das funções $z:\left[t_{0}-\Delta, t_{0}+\Delta\right] \rightarrow X$ tais que $z \in B V\left(\left[t_{0}-\Delta, t_{0}+\right.\right.$ $\Delta], X)$ e $\|z(t)-\widetilde{x}\| \leq\left|h(t)-h\left(t_{0}\right)\right|$, para $t \in\left[t_{0}-\Delta, t_{0}+\Delta\right]$. Note que $\mathcal{A}$ é fechado. De fato, seja $\left\{z_{n}\right\}_{n \in \mathbb{N}} \subset \mathcal{A}$ uma sequência tal que $z_{n} \rightarrow z$. Como

$$
\left\|z_{n}-z\right\|_{B V}=\left\|z_{n}\left(t_{0}\right)-z\left(t_{0}\right)\right\|+\operatorname{var}_{t_{0}-\Delta}^{t_{0}+\Delta}\left(z_{n}-z\right)
$$

então $\left\|z_{n}(t)-z(t)\right\| \leq\left\|z_{n}-z\right\|_{B V}$, para qualquer $t \in\left[t_{0}-\Delta, t_{0}+\Delta\right]$ e isto implica que $z_{n} \rightarrow z$ uniformemente.Portanto, dado $\varepsilon>0$, existe $n \in \mathbb{N}$ tal que

$$
\|z(t)-\widetilde{x}\| \leq\left\|z(t)-z_{n}(t)\right\|+\left\|z_{n}(t)-\widetilde{x}\right\| \leq \varepsilon+\left|h(t)-h\left(t_{0}\right)\right|
$$

Assim $\|z(t)-\widetilde{x}\| \leq\left|h(t)-h\left(t_{0}\right)\right|$ para qualquer $t \in\left[t_{0}-\Delta, t_{0}+\Delta\right]$ e, portanto, $z \in \mathcal{A}$, o que implica que $\mathcal{A}$ é fechado.

Para $s \in\left[t_{0}-\Delta, t_{0}+\Delta\right]$ e $z \in \mathcal{A}$, definamos

$$
T z(s)=\widetilde{x}+\int_{t_{0}}^{s} D G(z(\tau), t) .
$$

A integral $\int_{t_{0}}^{s} D G(z(\tau), t)$ existe pelo fato de $z$ ser de variação limitada e $G \in \mathcal{F}(\Omega, h)$ (veja o Corolário 1.27). Então, pelo Lema 1.19, vale

$$
\|T z(s)-\widetilde{x}\|=\left\|\int_{t_{0}}^{s} D G(z(\tau), t)\right\| \leq\left|h(s)-h\left(t_{0}\right)\right| .
$$

Portanto, $T z \in \mathcal{A}$, isto é, $T$ aplica $\mathcal{A}$ em $\mathcal{A}$.

Tomemos $t_{0}-\Delta \leq s_{1}<s_{2} \leq t_{0}+\Delta$ e $z_{1}, z_{2} \in \mathcal{A}$. Então,

$$
\begin{gathered}
\left\|T z_{2}\left(s_{2}\right)-T z_{1}\left(s_{2}\right)-\left[T z_{2}\left(s_{1}\right)-T z_{1}\left(s_{1}\right)\right]\right\|=\| \int_{t_{0}}^{s_{2}} D\left[G\left(z_{2}(\tau), t\right)-G\left(z_{1}(\tau), t\right)\right] \\
-\int_{t_{0}}^{s_{1}} D\left[G\left(z_{2}(\tau), t\right)-G\left(z_{1}(\tau), t\right)\right]\|=\| \int_{s_{1}}^{s_{2}} D\left[G\left(z_{2}(\tau), t\right)-G\left(z_{1}(\tau), t\right)\right] \|
\end{gathered}
$$




$$
\begin{gathered}
=\| \int_{s_{1}}^{s_{2}} D\left[G\left(z_{2}(\tau), t\right)-G\left(z_{1}(\tau), t\right)\right]-\sum_{i=1}^{n}\left[G\left(z_{2}\left(\tau_{i}\right), \alpha_{i}\right)-G\left(z_{1}\left(\tau_{i}\right), \alpha_{i}\right)-G\left(z_{2}\left(\tau_{i}\right), \alpha_{i-1}\right)\right. \\
\left.+G\left(z_{1}\left(\tau_{i}\right), \alpha_{i-1}\right)\right]+\sum_{i=1}^{n}\left[G\left(z_{2}\left(\tau_{i}\right), \alpha_{i}\right)-G\left(z_{1}\left(\tau_{i}\right), \alpha_{i}\right)-G\left(z_{2}\left(\tau_{i}\right), \alpha_{i-1}\right)+G\left(z_{1}\left(\tau_{i}\right), \alpha_{i-1}\right)\right] \| \\
\leq\left\|\sum_{i=1}^{n}\left[G\left(z_{2}\left(\tau_{i}\right), \alpha_{i}\right)-G\left(z_{1}\left(\tau_{i}\right), \alpha_{i}\right)-G\left(z_{2}\left(\tau_{i}\right), \alpha_{i-1}\right)+G\left(z_{1}\left(\tau_{i}\right), \alpha_{i-1}\right)\right]\right\|+\varepsilon \\
\leq \sum_{i=1}^{n}\left\|\left[G\left(z_{2}\left(\tau_{i}\right), \alpha_{i}\right)-G\left(z_{1}\left(\tau_{i}\right), \alpha_{i}\right)-G\left(z_{2}\left(\tau_{i}\right), \alpha_{i-1}\right)+G\left(z_{1}\left(\tau_{i}\right), \alpha_{i-1}\right)\right]\right\|+\varepsilon \\
\leq \sum_{i=1}^{n}\left\|z_{2}\left(\tau_{i}\right)-z_{1}\left(\tau_{i}\right)\right\|\left[h\left(\alpha_{i}\right)-h\left(\alpha_{i-1}\right)\right]+\varepsilon \\
\leq \sup _{\tau \in\left[s_{1}, s_{2}\right]}\left\|z_{2}(\tau)-z_{1}(\tau)\right\|\left(\sum_{i=1}^{n}\left[h\left(\alpha_{i}\right)-h\left(\alpha_{i-1}\right)\right]\right)+\varepsilon \\
\leq \sup _{\tau \in\left[t_{0}-\Delta, t_{0}+\Delta\right]}\left\|z_{2}(\tau)-z_{1}(\tau)\right\|\left[h\left(s_{2}\right)-h\left(s_{1}\right)\right]+\varepsilon
\end{gathered}
$$

para qualquer partição $\delta$-fina $\left\{\left(\tau_{i},\left[\alpha_{i-1}, \alpha_{i}\right]\right), i=1,2, \ldots, n\right\}$ de $\left[s_{1}, s_{2}\right]$.

Como $\varepsilon$ é arbitrário, temos

$$
\begin{aligned}
& \left\|T z_{2}\left(s_{2}\right)-T z_{1}\left(s_{2}\right)-\left[T z_{2}\left(s_{1}\right)-T z_{1}\left(s_{1}\right)\right]\right\| \leq \\
& \leq \sup _{\tau \in\left[t_{0}-\Delta, t_{0}+\Delta\right]}\left\|z_{2}(\tau)-z_{1}(\tau)\right\|\left[h\left(s_{2}\right)-h\left(s_{1}\right)\right] .
\end{aligned}
$$

Logo,

$$
\operatorname{var}_{t_{0}-\Delta}^{t_{0}+\Delta}\left(T z_{2}-T z_{1}\right) \leq \sup _{\tau \in\left[t_{0}-\Delta, t_{0}+\Delta\right]}\left\|z_{2}(\tau)-z_{1}(\tau)\right\|\left[h\left(s_{2}\right)-h\left(s_{1}\right)\right]
$$

Mas

$$
\sup _{\tau \in\left[t_{0}-\Delta, t_{0}+\Delta\right]}\left\|z_{2}(\tau)-z_{1}(\tau)\right\| \leq \operatorname{var}_{t_{0}-\Delta}^{t_{0}+\Delta}\left(z_{2}-z_{1}\right) .
$$

Logo, por (1.21) e (1.22), temos

$$
\begin{gathered}
\operatorname{var}_{t_{0}-\Delta}^{t_{0}+\Delta}\left(T z_{2}-T z_{1}\right) \leq \operatorname{var}_{t_{0}-\Delta}^{t_{0}+\Delta}\left(z_{2}-z_{1}\right)\left[h\left(s_{2}\right)-h\left(s_{1}\right)\right] \\
\leq \operatorname{var}_{t_{0}-\Delta}^{t_{0}+\Delta}\left(z_{2}-z_{1}\right)\left[h\left(t_{0}+\Delta\right)-h\left(t_{0}-\Delta\right)\right]
\end{gathered}
$$

pois $h$ é não decrescente e $t_{0}-\Delta \leq s_{1}<s_{2} \leq t_{0}+\Delta$.

Pela desigualdade na definição de $\mathcal{A}$, segue imediatamente que se $z_{i} \in \mathcal{A}, i=1,2$, então $z_{i}\left(t_{0}\right)=\widetilde{x}$. Temos, também, a igualdade $T\left(z_{i}\left(t_{0}\right)\right)=\widetilde{x}$. 
Logo,

$$
\operatorname{var}_{t_{0}-\Delta}^{t_{0}+\Delta}\left(T z_{2}-T z_{1}\right) \leq \operatorname{var}_{t_{0}-\Delta}^{t_{0}+\Delta}\left(z_{2}-z_{1}\right)\left[h\left(t_{0}+\Delta\right)-h\left(t_{0}-\Delta\right)\right]
$$

e isto implica que

$$
\left\|T z_{2}-T z_{1}\right\|_{B V} \leq\left\|z_{2}-z_{1}\right\|_{B V}\left[h\left(t_{0}+\Delta\right)-h\left(t_{0}-\Delta\right)\right]<\frac{1}{2}\left\|z_{2}-z_{1}\right\|_{B V} .
$$

Logo $T$ é uma contração e, pelo Teorema do Ponto Fixo de Banach, temos o resultado desejado.

Agora, consideremos $t_{0}$ como sendo um ponto de descontinuidade de $h$ e definamos

$$
\widetilde{h}(t)=\left\{\begin{array}{l}
h(t), \quad \text { se } t<t_{0} \\
h(t)-\left(h\left(t_{0}+\right)-h\left(t_{0}\right)\right), \quad \text { se } t \geq t_{0} .
\end{array}\right.
$$

Então a função $\widetilde{h}$ é contínua em $t_{0}$, não decrescente e contínua à esquerda.

Definindo, também,

$$
\widetilde{G}(x, t)=\left\{\begin{array}{l}
G(x, t), \quad \text { se } t<t_{0}, \\
G(x, t)-\left(G\left(\widetilde{x}, t_{0}+\right)-G\left(\widetilde{x}, t_{0}\right)\right), \quad \text { se } t \geq t_{0},
\end{array}\right.
$$

temos $\widetilde{G} \in \mathcal{F}(\Omega, \widetilde{h})$. Portanto, de modo análogo ao que fizemos anteriormente, concluímos que existe uma solução $z$ de $\frac{d z}{d \tau}=D \widetilde{G}(z(\tau), t) \operatorname{com} z\left(t_{0}\right)=\widetilde{x}+$. Daí, definindo

$$
x(t)=\left\{\begin{array}{lr}
\widetilde{x}, & \text { se } t=t_{0} \\
z(t), & \text { se } t>t_{0}
\end{array}\right.
$$

notamos que $x$ é solução de $\frac{d x}{d \tau}=D G(x, t) \operatorname{com} x\left(t_{0}\right)=\widetilde{x}$ e terminamos a prova.

\subsection{Dependência contínua para EDOGs}

Nesta seção, apresentaremos resultados sobre dependência contínua de soluções de equações diferenciais generalizadas com respeito aos dados iniciais.

Um outro resultado sobre dependência contínua com respeito aos valores iniciais será apresentado para uma classe restrita de equações diferenciais generalizadas com a qual 
poderemos associar uma classe de equações diferenciais funcionais impulsivas cujas funções assumem valores em $\mathbb{R}^{n}$. Este último, será apresentado no Capítulo 3.

No que segue, consideraremos $\Omega=O \times(a, b)$ e $h:[a, b] \rightarrow \mathbb{R}$ não decrescente e contínua à esquerda.

Lema 1.29 ([1], Lema 7.1). Suponhamos que $G_{k}: \Omega \rightarrow X$ pertença à classe $\mathcal{F}(\Omega, h)$, para $k=0,1,2, \ldots$, onde $h$ é não decrescente e contínua à esquerda. Seja $G_{k} \stackrel{k \rightarrow+\infty}{\longrightarrow} G_{0}$ em $\mathcal{F}(\Omega, h)$. Seja $\psi_{k} \in G([\alpha, \beta], X), k=1,2, \ldots,[\alpha, \beta] \subset[a, b]$ tal que $\left\|\psi_{k}-\psi_{0}\right\|_{\infty}=$ $\sup _{\alpha \leq t \leq \beta}\left\|\psi_{k}(t)-\psi_{0}(t)\right\| \stackrel{k \rightarrow+\infty}{\longrightarrow} 0$. Então

$$
\left\|\int_{\alpha}^{\beta} D G_{k}\left(\psi_{k}(\tau), s\right)-\int_{\alpha}^{\beta} D G_{0}\left(\psi_{0}(\tau), s\right)\right\| \stackrel{k \rightarrow+\infty}{\longrightarrow} 0 .
$$

Prova: Primeiramente, note que $\psi_{0} \in G([\alpha, \beta], X)$, pois $G([\alpha, \beta], X)$ com a norma do supremo é um espaço de Banach e $\psi_{0}$ é, por hipótese, o limite uniforme de funções regradas em $[a, b]$. Pelo Corolário 1.27, todas as integrais $\int_{\alpha}^{\beta} D G_{k}\left(\psi_{k}(\tau), s\right), k=0,1,2, \ldots$, existem.

Suponhamos que $\varepsilon>0$ seja dado. Então, como a função regrada $\psi_{0} \in G([\alpha, \beta], X)$ pode ser aproximada uniformemente por uma função escada finita, existe uma função escada finita $y:[\alpha, \beta] \rightarrow X$ tal que

$$
\left\|y-\psi_{0}\right\|_{\infty}=\sup _{\alpha \leq t \leq \beta}\left\|y(t)-\psi_{0}(t)\right\|<\varepsilon
$$

Como $\left\|\psi_{k}-\psi_{0}\right\|_{\infty} \stackrel{k \rightarrow+\infty}{\longrightarrow} 0$, existe um inteiro positivo $N_{0}$, tal que

$$
\left\|\psi_{k}-\psi_{0}\right\|_{\infty}<\varepsilon
$$

para todo $k>N_{0}$.

Suponhamos que $k>N_{0}$. Então

$$
\begin{gathered}
\left\|\int_{\alpha}^{\beta} D G_{k}\left(\psi_{k}(\tau), s\right)-\int_{\alpha}^{\beta} D G_{0}\left(\psi_{0}(\tau), s\right)\right\| \leq\left\|\int_{\alpha}^{\beta} D\left[G_{k}\left(\psi_{k}(\tau), s\right)-G_{k}\left(\psi_{0}(\tau), s\right)\right]\right\|+ \\
+\left\|\int_{\alpha}^{\beta} D\left[G_{k}\left(\psi_{0}(\tau), s\right)-G_{k}(y(\tau), s)\right]\right\|+\left\|\int_{\alpha}^{\beta} D\left[G_{k}(y(\tau), s)-G_{0}(y(\tau), s)\right]\right\|+ \\
+\left\|\int_{\alpha}^{\beta} D\left[G_{0}(y(\tau), s)-G_{0}\left(\psi_{0}(\tau), s\right)\right]\right\| .
\end{gathered}
$$

Vamos considerar o primeiro termo do lado direito da desigualdade em (1.23). 
Seja $\delta$ um calibre em $[a, b]$ correspondente a $\varepsilon$ da definição da integral $\int_{\alpha}^{\beta} D\left[G_{k}\left(\psi_{k}(\tau), s\right)-\right.$ $\left.G_{k}\left(\psi_{0}, s\right)\right]$ e seja $\left(\tau_{i},\left[s_{i-1}, s_{i}\right]\right)_{1 \leq i \leq p}$ uma partição $\delta$-fina de $[\alpha, \beta]$. Temos

$$
\begin{gathered}
\left\|\int_{\alpha}^{\beta} D\left[G_{k}\left(\psi_{k}(\tau), s\right)-G_{k}\left(\psi_{0}(\tau), s\right)\right]\right\| \\
\leq \| \int_{\alpha}^{\beta} D\left[G_{k}\left(\psi_{k}(\tau), s\right)-G_{k}\left(\psi_{0}(\tau), s\right)\right]- \\
-\sum_{i=1}^{p}\left[\left(G_{k}\left(\psi_{k}\left(\tau_{i}\right), s_{i}\right)-G_{k}\left(\psi_{k}\left(\tau_{i}\right), s_{i-1}\right)\right)-\left(G_{k}\left(\psi_{0}\left(\tau_{i}\right), s_{i}\right)-G_{k}\left(\psi_{0}\left(\tau_{i}\right), s_{i-1}\right)\right)\right] \|+ \\
\left\|\sum_{i=1}^{p}\left[\left(G_{k}\left(\psi_{k}\left(\tau_{i}\right), s_{i}\right)-G_{k}\left(\psi_{k}\left(\tau_{i}\right), s_{i-1}\right)\right)-\left(G_{k}\left(\psi_{0}\left(\tau_{i}\right), s_{i}\right)-G_{k}\left(\psi_{0}\left(\tau_{i}\right), s_{i-1}\right)\right)\right]\right\| \leq \\
<\varepsilon+\sum_{i=1}^{p}\left\|G_{k}\left(\psi_{k}\left(\tau_{i}\right), s_{i}\right)-G_{k}\left(\psi_{k}\left(\tau_{i}\right), s_{i-1}\right)-G_{k}\left(\psi_{0}\left(\tau_{i}\right), s_{i}\right)+G_{k}\left(\psi_{0}\left(\tau_{i}\right), s_{i-1}\right)\right\| \leq \\
(1.16) \\
\leq \quad+\sum_{i=1}^{p}\left\|\psi_{k}\left(\tau_{i}\right)-\psi_{0}\left(\tau_{i}\right)\right\|\left|h\left(s_{i}\right)-h\left(s_{i-1}\right)\right| \leq \\
\leq \varepsilon+\left\|\psi_{k}-\psi_{0}\right\|_{\infty} \sum_{i=1}^{p}\left[h\left(s_{i}\right)-h\left(s_{i-1}\right)\right] \leq \\
\leq \varepsilon+\varepsilon[h(\beta)-h(\alpha)]=\varepsilon(1+[h(\beta)-h(\alpha)]) .
\end{gathered}
$$

Para o segundo e quarto termos do lado direito de (1.23), podemos mostrar analogamente que

$$
\left\|\int_{\alpha}^{\beta} D\left[G_{k}\left(\psi_{0}(\tau), s\right)-G_{k}(y(\tau), s)\right]\right\|<\varepsilon(1+[h(\beta)-h(\alpha)])
$$

e

$$
\left\|\int_{\alpha}^{\beta} D\left[G_{0}(y(\tau), s)-G_{0}\left(\psi_{0}(\tau), s\right)\right]\right\|<\varepsilon(1+[h(\beta)-h(\alpha)]),
$$

ou seja, temos

$$
\begin{gathered}
\left\|\int_{\alpha}^{\beta} D G_{k}\left(\psi_{k}(\tau), s\right)-\int_{\alpha}^{\beta} D G_{0}\left(\psi_{0}(\tau), s\right)\right\|< \\
<3 \varepsilon(1+[h(\beta)-h(\alpha)])+\left\|\int_{\alpha}^{\beta} D\left[G_{k}(y(\tau), s)-G_{0}(y(\tau), s)\right]\right\| .
\end{gathered}
$$

Agora, vamos considerar a integral $\int_{\alpha}^{\beta} D\left[G_{k}(y(\tau), s)-G_{0}(y(\tau), s)\right]$.

Como $y:[\alpha, \beta] \rightarrow X$ é uma função escada finita, existe um número finito de pontos 
$\alpha=r_{0}<r_{1}<r_{2}<\cdots<r_{p-1}<r_{p}=\beta$ tais que, para $\tau \in\left(r_{j-1}, r_{j}\right), j=1,2, \ldots, p$, temos $y(\tau)=c_{j} \in X$, isto é, $y$ assume um valor constante $c_{j}$ em cada intervalo aberto $\left(r_{j-1}, r_{j}\right)$, $j=1,2, \ldots, p$. Neste caso, uma fórmula explícita para a integral $\int_{\alpha}^{\beta} D G_{k}(y(\tau), s)$, para cada $k=0,1,2, \ldots$, pode ser dada por

$$
\int_{\alpha}^{\beta} D G_{k}(y(\tau), s)=\sum_{j=1}^{p} \int_{r_{j-1}}^{r_{j}} D G_{k}(y(\tau), s)
$$

e, usando o Teorema 1.9 e escolhendo uma partição de $\left[r_{j-1}, r_{j}\right]$, temos

$$
\begin{gathered}
\int_{r_{j-1}}^{r_{j}} D G_{k}(y(\tau), t)=G_{k}\left(c_{j}, r_{j}-\right)-G_{k}\left(c_{j}, r_{j-1}+\right)+G_{k}\left(y\left(r_{j-1}\right), r_{j-1}+\right)- \\
-G_{k}\left(y\left(r_{j-1}\right), r_{j-1}\right)-G_{k}\left(y\left(r_{j}\right), r_{j}-\right)+G_{k}\left(y\left(r_{j}\right), r_{j}\right) .
\end{gathered}
$$

Olhando a diferença entre $G_{k}$ e $G_{0}$ na expressão do lado direito na última igualdade, obtemos

$$
\lim _{k \rightarrow \infty} \int_{r_{j-1}}^{r_{j}} D\left[G_{k}(y(\tau), t)-G_{0}(y(\tau), t)\right]=0,
$$

pois como $G_{k} \in \mathcal{F}(\Omega, h)$, vale

$$
\left\|G_{k}\left(x, t_{2}\right)-G_{k}\left(x, t_{1}\right)\right\| \leq\left|h\left(t_{2}\right)-h\left(t_{1}\right)\right|
$$

para quaisquer $\left(x, t_{1}\right),\left(x, t_{2}\right) \in \Omega$, e isto implica que $\lim _{\rho \rightarrow 0+} G_{k}(x, t+\rho)=G_{k}(x, t+)$ e $\lim _{\rho \rightarrow 0+} G_{k}(x, t-\rho)=G_{k}(x, t-)$ para todo $(x, t) \in \Omega$ uniformemente com respeito a $k=0,1, \ldots$.

Como por hipóteste $G_{k} \stackrel{k \rightarrow+\infty}{\longrightarrow} G_{0}$, então

$$
\begin{aligned}
\lim _{k \rightarrow+\infty} G_{k}(x, t+) & =\lim _{k \rightarrow+\infty} \lim _{\rho \rightarrow 0+} G_{k}(x, t+\rho)= \\
& =\lim _{\rho \rightarrow 0+} \lim _{k \rightarrow+\infty} G_{k}(x, t+\rho)= \\
& =\lim _{\rho \rightarrow 0+} G_{0}(x, t+\rho)=G_{0}(x, t+),
\end{aligned}
$$

desde que $(x, t) \in \Omega$.

Finalmente, como $\varepsilon>0$ pode ser escolhido suficientemente pequeno, obtemos o resultado desejado.

O corolário abaixo segue imediatamente do lema anterior, como podemos notar.

Corolário 1.30 ([1], Corolário 7.2). Seja $G_{k} \stackrel{k \rightarrow+\infty}{\longrightarrow} G_{0}$ pertencentes à classe $\mathcal{F}(\Omega, h)$, 
onde $h$ é não decrescente e contínua à esquerda. Seja $\psi_{k} \in B V([\alpha, \beta], X), k=0,1,2, \ldots$, $[\alpha, \beta] \subset(a, b)$, tal que $\left\|\psi_{k}-\psi_{0}\right\|_{B V} \stackrel{k \rightarrow+\infty}{\longrightarrow} 0$. Então

$$
\left\|\int_{\alpha}^{\beta} D G_{k}\left(\psi_{k}(\tau), s\right)-\int_{\alpha}^{\beta} D G_{0}\left(\psi_{0}(\tau), s\right)\right\| \stackrel{k \rightarrow+\infty}{\longrightarrow} 0 .
$$

Prova: Levando em consideração que $B V([\alpha, \beta], X) \subset G([\alpha, \beta], X)$ e que, para todo $t \in[\alpha, \beta]$, temos

$$
\begin{aligned}
\left\|\psi_{k}(t)-\psi_{0}(t)\right\| & \leq\left\|\psi_{k}(\alpha)-\psi_{0}(\alpha)\right\|+\left\|\psi_{k}(t)-\psi_{0}(t)-\left[\psi_{k}(\alpha)-\psi_{0}(\alpha)\right]\right\| \leq \\
& \leq\left\|\psi_{k}(\alpha)-\psi_{0}(\alpha)\right\|+\operatorname{var}_{\alpha}^{t}\left(\psi_{k}-\psi_{0}\right) \leq \\
& \leq\left\|\psi_{k}(\alpha)-\psi_{0}(\alpha)\right\|+\operatorname{var}_{\alpha}^{\beta}\left(\psi_{k}-\psi_{0}\right)=\left\|\psi_{k}-\psi_{0}\right\|_{B V},
\end{aligned}
$$

podemos ver facilmente que $\left\|\psi_{k}-\psi_{0}\right\|_{\infty} \stackrel{k \rightarrow+\infty}{\longrightarrow} 0$ e o resultado segue do Lema 1.29.

O teorema seguinte é um resultado sobre dependência contínua que generaliza o Teorema 8.2 encontrado em [26] para valores a espaço de Banach. Sua demonstração é completamente diferente da prova apresentada em [26] que usa o Princípio da Escolha de Helly.

Proposição 1.31 ([1], Proposição 7.3). Suponhamos que $G_{k}: \Omega \rightarrow X$ pertença a classe $\mathcal{F}(\Omega, h)$, para $k=0,1,2, \ldots$, onde $h$ é não decrescente e contínua à esquerda e que

$$
\lim _{k \rightarrow+\infty} G_{k}(x, t)=G_{0}(x, t)
$$

para $(x, t) \in \Omega$. Sejam $x_{k}:[\alpha, \beta] \rightarrow X, k=1,2, \ldots$, soluções da equação diferencial generalizada

$$
\frac{d x}{d \tau}=D G_{k}(x, t)
$$

em $[\alpha, \beta]$ tal que

$$
\lim _{k \rightarrow+\infty} x_{k}(s)=x_{0}(s), \quad s \in[\alpha, \beta],
$$

$e(x(s), s) \in \Omega$ para $s \in[\alpha, \beta]$. Então $x_{0}:[\alpha, \beta] \rightarrow X$ satisfaz as seguintes condições:

i) $\left\|x_{0}\left(s_{2}\right)-x_{0}\left(s_{1}\right)\right\| \leq h\left(s_{2}\right)-h\left(s_{1}\right)$, se $s_{1} \leq s_{2}$ e $s_{1}, s_{2} \in[\alpha, \beta]$;

ii) $\lim _{k \rightarrow+\infty} x_{k}(s)=x_{0}(s)$ uniformemente em $[\alpha, \beta]$;

iii) $x_{0}$ é uma solução da equação diferencial generalizada $\frac{d x}{d \tau}=D G_{0}(x, t)$ em $[\alpha, \beta]$. 
Prova: Suponhamos que $\alpha \leq s_{1} \leq s_{2} \leq \beta$. Então para todo $k \in \mathbb{N}$, temos

$$
\left\|x_{0}\left(s_{2}\right)-x_{0}\left(s_{1}\right)\right\| \leq\left\|x_{0}\left(s_{2}\right)-x_{k}\left(s_{2}\right)\right\|+\left\|x_{k}\left(s_{2}\right)-x_{k}\left(s_{1}\right)\right\|+\left\|x_{k}\left(s_{1}\right)-x_{0}\left(s_{1}\right)\right\| \text {. }
$$

Seja $\varepsilon>0$ arbitrário. Por (1.24), existe um $\ell \in \mathbb{N}$ tal que, para $k>\ell$, temos

$$
\left\|x_{k}\left(s_{2}\right)-x_{0}\left(s_{2}\right)\right\|<\frac{\varepsilon}{2} \quad \text { e } \quad\left\|x_{k}\left(s_{1}\right)-x_{0}\left(s_{1}\right)\right\|<\frac{\varepsilon}{2} .
$$

Usando o Lema 1.22, temos

$$
\left\|x_{k}\left(s_{2}\right)-x_{k}\left(s_{1}\right)\right\| \leq h\left(s_{2}\right)-h\left(s_{1}\right), \quad k=1,2,3, \ldots
$$

Logo

$$
\left\|x_{0}\left(s_{2}\right)-x_{0}\left(s_{1}\right)\right\|<\varepsilon+h\left(s_{2}\right)-h\left(s_{1}\right) .
$$

Como $\varepsilon>0$ pode ser tomado suficientemente pequeno, obtemos

$$
\left\|x_{0}\left(s_{2}\right)-x_{0}\left(s_{1}\right)\right\| \leq h\left(s_{2}\right)-h\left(s_{1}\right)
$$

e isto implica $i$ ).

Para provar $i i)$, vamos supor primeiramente que $[\alpha, \beta]$ não contém pontos de descontinuidades da função $h$, ou seja, $h:[\alpha, \beta] \rightarrow \mathbb{R}$ é contínua. Pelo Teorema de Heine-Borel, $h$ é uniformemente contínua em $[\alpha, \beta]$. Isto significa que para todo $\varepsilon>0$, existe um $\delta>0$ tal que $|h(s)-h(t)|<\varepsilon$, sempre que $|s-t|<\delta$.

Seja $\varepsilon>0$ arbitrário e seja $\delta>0$ correspondente a $\varepsilon$ na definição de continuidade uniforme. Então intervalos da forma $(t-\delta, t+\delta)$, com $t \in[\alpha, \beta]$, cobrem $[\alpha, \beta]$. Como $[\alpha, \beta]$ é compacto, existe um conjunto finito de pontos $r_{1}, \ldots, r_{\ell}$ tal que $[\alpha, \beta]$ é coberto por um número finito de intervalos $\left(r_{j}-\delta, r_{j}+\delta\right), j=1, \ldots, \ell$.

Tomemos $k^{*} \in \mathbb{N}$ tal que (por (1.24)), para $k>k^{*}$, tenhamos

$$
\left\|x_{k}\left(r_{j}\right)-x_{0}\left(r_{j}\right)\right\|<\varepsilon
$$

para todo $j=1, \ldots, \ell$. Seja $s \in[\alpha, \beta]$ dado. Então existe $j \in\{1, \ldots, \ell\}$ tal que $s \in\left(r_{j}-\delta, r_{j}+\delta\right)$. Logo, para $k>k^{*}$, temos

$$
\begin{aligned}
\left\|x_{k}(s)-x_{0}(s)\right\| & \leq\left\|x_{k}(s)-x_{k}\left(r_{j}\right)\right\|+\left\|x_{k}\left(r_{j}\right)-x_{0}\left(r_{j}\right)\right\|+\left\|x_{0}\left(r_{j}\right)-x_{0}(s)\right\| \leq \\
& \leq\left|h(s)-h\left(r_{j}\right)\right|+\varepsilon+\left|h(s)-h\left(r_{j}\right)\right| \leq 3 \varepsilon .
\end{aligned}
$$


Como isto pode ser feito para todo $s \in[\alpha, \beta]$, obtemos $i i)$ neste caso.

Agora, sejam $\alpha<t_{1}<\ldots<t_{m}<\beta$ os pontos de descontinuidades de $h$ em $[\alpha, \beta]$.

Suponhamos que $\varepsilon>0$ seja dado. Por (1.24), existe um $k^{*} \in \mathbb{N}$ tal que, para todo $j \in\{1,2, \ldots, m\}$, obtemos

$$
\left\|x_{k}\left(t_{j}\right)-x_{0}\left(t_{j}\right)\right\|<\varepsilon
$$

sempre que $k>k^{*}$.

Seja $[c, d]$ um dos intervalos $\left[\alpha, t_{1}\right],\left[t_{1}, t_{2}\right], \ldots,\left[t_{m-1}, t_{m}\right]$ ou $\left[t_{m}, \beta\right]$, e definamos

$$
h^{*}(s)= \begin{cases}h(s), & s \in(c, d] \\ h(a+), & s=c .\end{cases}
$$

Pelas hipóteses de $h$, a função $h^{*}:[c, d] \rightarrow \mathbb{R}$ é não decrescente e contínua.

Para cada $k=0,1, \ldots$, seja $x_{k}^{*}(c)=x_{k}(c+)$ e $x_{k}^{*}(s)=x_{k}(s), s \in(c, d]$. Então, por (1.24), temos

$$
\begin{gathered}
\lim _{k \rightarrow+\infty} x_{k}^{*}(s)=x_{0}^{*}(s), \quad s \in(c, d], \\
\lim _{k \rightarrow+\infty} x_{k}^{*}(c)=\lim _{k \rightarrow+\infty} x_{k}(c+)=x_{0}(c+)=x_{0}^{*}(c)
\end{gathered}
$$

e

$$
\left\|x_{k}^{*}\left(s_{2}\right)-x_{k}^{*}\left(s_{1}\right)\right\| \leq\left|h^{*}\left(s_{2}\right)-h^{*}\left(s_{1}\right)\right|
$$

para $c \leq s_{1} \leq s_{2} \leq d$.

Usando a parte anterior, $x_{k}^{*} \stackrel{k \rightarrow+\infty}{\longrightarrow} x_{0}^{*}$ uniformemente em $[c, d]$. Portanto, para todo $\varepsilon>0$, existe $k_{*} \in \mathbb{N}$ tal que

$$
\left\|x_{k}(s)-x_{0}(s)\right\|=\left\|x_{k}^{*}(s)-x_{0}^{*}(s)\right\|<\varepsilon
$$

sempre que $s \in(c, d],\left\|x_{k}(c)-x_{0}(c)\right\|<\varepsilon, k>k_{*}$ e $x_{k} \stackrel{k \rightarrow+\infty}{\longrightarrow} x_{0}$ uniformemente em $[c, d]$. Como isto pode ser feito para um número finito de intervalos do tipo $[c, d]$, obtemos $i i)$ e sua forma geral como afirmada na conclusão do teorema.

Agora, vamos provar iii). Pela definição de uma solução da equação diferencial generalizada $\frac{d x}{d \tau}=D G_{k}(x, t), k=1,2,3, \ldots$, temos

$$
x_{k}\left(s_{2}\right)-x_{k}\left(s_{1}\right)=\int_{s_{1}}^{s_{2}} D G_{k}\left(x_{k}(\tau), t\right)
$$

para todo $s_{1}, s_{2} \in[\alpha, \beta]$. Pelo Corolário 1.30, temos 


$$
\left\|\int_{s_{1}}^{s_{2}} D G_{k}\left(x_{k}(\tau), s\right)-\int_{s_{1}}^{s_{2}} D G_{0}\left(x_{0}(\tau), s\right)\right\| \stackrel{k \rightarrow+\infty}{\longrightarrow} 0,
$$

para todo $s_{1}, s_{2} \in[\alpha, \beta]$. Usando (1.25), temos

$$
\begin{gathered}
\left\|x_{0}\left(s_{2}\right)-x_{0}\left(s_{1}\right)-\int_{s_{1}}^{s_{2}} D G_{0}\left(x_{0}(\tau), t\right)\right\| \leq \\
=\left\|x_{k}\left(s_{2}\right)-x_{0}\left(s_{2}\right)\right\|+\left\|x_{k}\left(s_{1}\right)-x_{0}\left(s_{1}\right)\right\|+\left\|\int_{s_{1}}^{s_{2}} D G_{k}\left(x_{k}(\tau), s\right)-\int_{s_{1}}^{s_{2}} D G_{0}\left(x_{0}(\tau), s\right)\right\| .
\end{gathered}
$$

Então, quando $k \rightarrow+\infty$, obtemos

$$
x_{0}\left(s_{2}\right)-x_{0}\left(s_{1}\right)=\int_{s_{1}}^{s_{2}} D G_{0}\left(x_{0}(\tau), t\right)
$$

para todo $s_{1}, s_{2} \in[\alpha, \beta]$. Portanto $x$ é uma solução da equação diferencial generalizada $\frac{d x}{d \tau}=D G_{0}(x, t)$ em $[\alpha, \beta]$ e terminamos a prova do teorema.

A seguir, introduziremos algumas notações.

Seja $h_{0}:[a, b] \rightarrow \mathbb{R}$ uma função não decrescente e contínua à esquerda em $[a, b]$. Suponhamos que $\eta>0$ seja dado. Se $[\alpha, \beta] \subset(a, b)$, então denotaremos

$$
D=D\left(\eta, h_{0},[\alpha, \beta]\right)=\left\{t \in[\alpha, \beta] ; h_{0}(t+)-h_{0}(t)>\eta\right\}
$$

Como $h_{0}$ é contínua à esquerda, então para todo $\eta>0$, o conjunto $D$ é finito. $\mathrm{O}$ fato de existirem os limites laterais da função $h_{0}$ em todos os pontos de $[\alpha, \beta]$ implica a existência de uma função calibre $\delta_{1}:[\alpha, \beta] \rightarrow(0,+\infty)$ com as seguintes propriedades:

- Se $t \notin D$, então

$$
h_{0}\left(t+\delta_{1}(t)\right)-h_{0}\left(t-\delta_{1}(t)\right)<\eta, \text { para } t \in(\alpha, \beta) \text {. }
$$

Além disso, se $\alpha \notin D$, então

$$
h_{0}\left(\alpha+\delta_{1}(\alpha)\right)-h_{0}(\alpha)<\eta
$$

e se $\beta \notin D$,

$$
h_{0}\left(\beta+\delta_{1}(\beta)\right)-h_{0}(\beta)<\eta \text {. }
$$

- Se $t \in D$, então

$$
h_{0}\left(t+\delta_{1}(t)\right)-h_{0}(t+)<\eta, t \in[\alpha, \beta),
$$


$\mathrm{e}$

$$
h_{0}(t)-h_{0}\left(t-\delta_{1}(t)\right)<\eta, t \in(\alpha, \beta] .
$$

Em particular, se $t \in D$ for igual a $\alpha$ ou a $\beta$, então somente uma destas duas últimas desigualdades será requerida.

Definamos, também, uma função calibre $\delta_{2}:[\alpha, \beta] \rightarrow(0,+\infty)$ da maneira abaixo

$$
\delta_{2}(\tau)=\left\{\begin{array}{l}
\operatorname{dist}(\tau, D), \quad \text { para } \tau \notin D \\
1, \quad \text { para } \tau \in D
\end{array}\right.
$$

e seja

$$
\delta_{\eta, h_{0}}(\tau)=\min \left\{\delta_{1}(\tau), \delta_{2}(\tau)\right\}, \quad \text { para } \tau \in[\alpha, \beta]
$$

Então a função $\delta_{\eta, h_{0}}:[\alpha, \beta] \rightarrow(0,+\infty)$ também é um calibre em $[\alpha, \beta]$, já que $\delta_{1}$ e $\delta_{2}$ o são. Note que, pelo Lema de Cousin (Lema 1.1), o conjunto das partições $\delta_{\eta, h_{0}}$-finas de $[\alpha, \beta]$ é não vazio. Suponhamos, então, que

$$
A=\left\{\alpha_{0}, \tau_{1}, \alpha_{1}, \ldots, \alpha_{k-1}, \tau_{k}, \alpha_{k}\right\}
$$

seja uma partição $\delta_{\eta, h_{0}}$-fina de $[\alpha, \beta]$. Então

$$
D \subset\left\{\tau_{1}, \tau_{2}, \ldots, \tau_{k}\right\}
$$

pois $\delta_{\eta, h_{0}}(\tau)<\delta_{1}(\tau)$ para $\tau \in[\alpha, \beta]$, pelas definições de $\delta_{2}$ e de $\delta_{\eta, h_{0}}$. Isto pode ser mostrado da mesma forma como na demonstração do Teorema 1.7.

Como $A$ é uma partição $\delta_{\eta, h_{0}}$-fina, então pelas propriedades do calibre $\delta_{\eta, h_{0}}$, temos as seguintes propriedades

- Se $\tau_{m} \notin D$, então

$$
h_{0}\left(\alpha_{m}\right)-h_{0}\left(\alpha_{m-1}\right)<\eta
$$

- Se $\tau_{m} \in D$, então

$$
\begin{aligned}
& h_{0}\left(\alpha_{m}\right)-h_{0}\left(\tau_{m}+\right)<\eta \\
& h_{0}\left(\tau_{m}\right)-h_{0}\left(\alpha_{m-1}\right)<\eta .
\end{aligned}
$$

A propriedade (1.34) segue facilmente de (1.26), (1.27) e (1.28).

Analogamente (1.35) é uma consequência imediata de (1.29) e (1.30). Como o limite lateral à direita de $h_{0}$ existe em todo ponto de $[\alpha, \beta)$, podemos ver que a partição $\delta_{\eta, h_{0}}$-fina 
$A$ de $[\alpha, \beta]$ pode ser escolhida de modo que

$$
\alpha_{m}>\tau_{m}, \quad \text { quando } \tau_{\mathrm{m}} \in \mathrm{D} \cap[\alpha, \beta)
$$

Portanto, pelas considerações feitas anteriormente, afirmamos que para toda função não decrescente $h_{0}:[\alpha, \beta] \rightarrow \mathbb{R}$ que é contínua à esquerda e para todo $\eta>0$, existe uma partição do intervalo $[\alpha, \beta]$ que satisfaz (1.33), (1.34), (1.35) e (1.36).

Como o conjunto dos pontos de continuidade de $h_{0}$ é denso em $[\alpha, \beta]$ e todas as desigualdades em (1.34) até (1.36) estão satisfeitas para estes pontos, a partição pode ser escolhida de modo que os pontos $\alpha_{1}, \ldots, \alpha_{k-1}$ sejam pontos de continuidade de $h_{0}$.

O lema abaixo é uma generalização do Lema 8.4 encontrado em [26] e trata da existência da integral $\int_{\alpha}^{\beta} D G(x(\tau), t)$ quando $G \in \mathcal{F}(\Omega, h)$, onde $[\alpha, \beta] \subset(a, b)$.

Proposição 1.32 ([26], Lema 8.4). Suponhamos que as funções $h, h_{0}:[a, b] \rightarrow \mathbb{R}$ sejam não decrescentes e contínuas à esquerda. Suponhamos, também, que $G: \Omega \rightarrow X$ seja uma função pertencente a $\mathcal{F}(\Omega, h)$. Se $x:[\alpha, \beta] \rightarrow X,[\alpha, \beta] \subset(a, b)$, for tal que $(x(\tau), \tau) \in \Omega$ para $\tau \in[\alpha, \beta] e$

$$
\left\|x\left(t_{2}\right)-x\left(t_{1}\right)\right\| \leq h_{0}\left(t_{2}\right)-h_{0}\left(t_{1}\right), \quad \alpha \leq t_{1} \leq t_{2} \leq \beta
$$

então a integral $\int_{\alpha}^{\beta} D G(x(\tau), t)$ existirá. Se, além disso, $\eta>0$ for arbitrário e $A=$ $\left\{\alpha_{0}, \tau_{1}, \alpha_{1}, \ldots, \alpha_{k-1}, \tau_{k}, \alpha_{k}\right\}$ for uma partição de $[\alpha, \beta]$ que satisfaz (1.33), (1.34), (1.35) e (1.36), então

$$
\left\|\int_{\alpha}^{\beta} D G(x(\tau), t)-\sum_{m=1}^{k} S_{m}\right\| \leq \eta\left[h(\beta)-h(\alpha)+h_{0}(\beta)-h_{0}(\alpha)\right]
$$

onde $S_{m}=G\left(x\left(\tau_{m}\right), \alpha_{m}\right)-G\left(x\left(\tau_{m}\right), \alpha_{m-1}\right)$, se $\tau_{m} \notin D, e$

$$
S_{m}=G\left(x\left(\tau_{m}+\right), \alpha_{m}\right)-G\left(x\left(\tau_{m}+\right), \tau_{m}+\right)+G\left(x\left(\tau_{m}\right), \tau_{m+}\right)-G\left(x\left(\tau_{m}\right), \alpha_{m-1}\right),
$$

se $\tau_{m} \in D$.

Prova: Como $G \in \mathcal{F}(\Omega, h)$, pela hipótese (1.37), a função $x$ é de variação limitada em $[\alpha, \beta]$. Desta forma, todas as hipóteses do Corolário 1.27 estão satisfeitas e, portanto, a integral $\int_{\alpha}^{\beta} D G(x(\tau), t)$ existe e a primeira parte do lema está provada.

Para provar a segunda parte, consideremos $\eta>0$ dado e suponhamos que $A$ seja uma partição de $[\alpha, \beta]$ que satisfaz (1.33), (1.34), (1.35) e (1.36). Assim, pelo Teorema 1.6, a integral $\int_{\alpha_{m-1}}^{\alpha_{m}} D G(x(\tau), t)$ existe para todo $m=1, . ., k$. 
Seja $m \in\{1, \ldots, k\}$ fixo e suponhamos que $\tau_{m} \in D$. Então, para todo $\varepsilon>0$, existem calibres $\delta^{-}$e $\delta^{+}$em $\left[\alpha_{m-1}, \tau_{m}\right]$ e $\left[\tau_{m}, \alpha_{m}\right]$ respectivamente, tais que

$$
\| \int_{\alpha_{m-1}}^{\tau_{m}} D G\left(x(\tau, t)-S\left(A^{-}\right) \| \leq \frac{\varepsilon}{2}\right.
$$

para toda partição $\delta^{-}$-fina $A^{-}$de $\left[\alpha_{m-1}, \tau_{m}\right]$, onde $S\left(A^{-}\right)$é a soma de Riemann correspondente a $A^{-}$, e

$$
\left\|\int_{\tau_{m}}^{\alpha_{m}} D G(x(\tau), t)-S\left(A^{+}\right)\right\| \leq \frac{\varepsilon}{2}
$$

para toda partição $\delta^{+}$-fina $A^{-}$de $\left[\tau_{m}, \alpha_{m}\right]$, onde $S\left(A^{+}\right)$é a soma de Riemann correspondente a $A^{+}$.

Das desigualdades acima, obtemos

$$
\begin{gathered}
\left\|\int_{\alpha_{m-1}}^{\alpha_{m}} D G(x(\tau), t)-S\left(A^{-}\right)-S\left(A^{+}\right)\right\|= \\
=\left\|\int_{\alpha_{m-1}}^{\tau_{m}} D G(x(\tau), t)+\int_{\tau_{m}}^{\alpha_{m}} D G(x(\tau), t)-S\left(A^{-}\right)-S\left(A^{+}\right)\right\| \leq \\
\leq\left\|\int_{\alpha_{m-1}}^{\tau_{m}} D G(x(\tau), t)-S\left(A^{-}\right)\right\|+\left\|\int_{\tau_{m}}^{\alpha_{m}} D G(x(\tau), t)-S\left(A^{+}\right)\right\| \leq \frac{\varepsilon}{2}+\frac{\varepsilon}{2}=\varepsilon .
\end{gathered}
$$

Sem perda de generalidade, podemos supor que o valor $\delta^{+}\left(\tau_{m}\right)>0$ seja tal que

$$
h(\alpha)-h\left(\tau_{m}+\right)<\eta
$$

para todo $\alpha \in\left(\tau_{m}, \tau_{m}+\delta^{+}\left(\tau_{m}\right)\right)$. Suponhamos, ainda, que

$$
A^{-}=\left\{\bar{\alpha}_{0}, \overline{\tau_{1}}, \bar{\alpha}_{1}, \ldots, \alpha_{\overline{l-1}}^{-}, \bar{\tau}_{l}, \bar{\alpha}_{l}\right\}
$$

seja uma partição $\delta^{-}$-fina de $\left[\alpha_{m-1}, \tau_{m}\right]$ satisfazendo

$$
\alpha_{m-1}=\overline{\alpha_{0}} \leq \overline{\tau_{1}} \leq \overline{\alpha_{1}} \leq \ldots \leq \alpha_{\overline{l-1}} \leq \bar{\tau}_{l}=\bar{\alpha}_{l}=\tau_{m}
$$

Suponhamos, também, que

$$
A^{+}=\left\{\bar{\alpha}_{l}, \bar{\tau}_{l+1}, \bar{\alpha}_{l+1}, \ldots, \bar{\alpha}_{l+s-1}, \bar{\tau}_{l+s}, \bar{\alpha}_{l+s}\right\}
$$


seja uma partição $\delta^{+}$-fina de $\left[\tau_{m}, \alpha_{m}\right]$ satisfazendo

$$
\tau_{m}=\bar{\alpha}_{l}=\bar{\tau}_{l+1} \leq \bar{\alpha}_{l+1} \leq \bar{\tau}_{l+2} \ldots \leq \bar{\alpha}_{l+s-1} \leq \bar{\tau}_{l+s}=\bar{\alpha}_{l+s}=\alpha_{m}
$$

Então, pelas propriedades da classe $\mathcal{F}(\Omega, h)$, temos

$$
\begin{aligned}
& \left\|S\left(A^{-}\right)-S\left(A^{+}\right)-S_{m}\right\| \\
& =\| \sum_{j=1}^{l}\left[G\left(x\left(\bar{\tau}_{j}\right), \bar{\alpha}_{j}\right)-G\left(x\left(\bar{\tau}_{j}\right), \bar{\alpha}_{j-1}\right)\right]-\left(G\left(x\left(\tau_{m}\right), \tau_{m}+\right)-G\left(x\left(\tau_{m}\right), \alpha_{m-1}\right)\right)+ \\
& +\sum_{j=l+1}^{l+s}\left[G\left(x\left(\bar{\tau}_{j}\right), \bar{\alpha}_{j}\right)-G\left(x\left(\bar{\tau}_{j}\right), \bar{\alpha}_{j-1}\right)\right]-\left(G\left(x\left(\tau_{m}+\right), \alpha_{m}\right)-G\left(x\left(\tau_{m}+\right), \tau_{m}+\right)\right) \| \\
& =\| \sum_{j=1}^{l}\left[G\left(x\left(\bar{\tau}_{j}\right), \bar{\alpha}_{j}\right)-G\left(x\left(\bar{\tau}_{j}\right), \bar{\alpha}_{j-1}\right)\right]-G\left(x\left(\tau_{m}\right), \bar{\alpha}_{l}\right)+G\left(x\left(\tau_{m}\right), \bar{\alpha}_{l}\right)- \\
& -G\left(x\left(\tau_{m}\right), \bar{\alpha}_{0}\right)+G\left(x\left(\tau_{m}\right), \bar{\alpha}_{0}\right)+G\left(x\left(\bar{\tau}_{l+1}\right), \bar{\alpha}_{l+1}\right)-G\left(x\left(\bar{\tau}_{l+1}\right), \alpha_{l}\right)- \\
& \left.-G\left(x\left(\tau_{m}\right), \tau_{m}+\right)+G\left(x\left(\tau_{m}\right), \alpha_{m-1}\right)\right)-G\left(x\left(\tau_{m}+\right), \alpha_{m}\right)-G\left(x\left(\tau_{m}+\right), \tau_{m}+\right)+ \\
& +G\left(x\left(\tau_{m}+\right), \bar{\alpha}_{l+s}\right)-G\left(x\left(\tau_{m}+\right), \bar{\alpha}_{l+s}\right)- \\
& -G\left(x\left(\tau_{m}+\right), \bar{\alpha}_{l+1}\right)+G\left(x\left(\tau_{m}+\right), \bar{\alpha}_{l+1}\right)+\sum_{j=l+2}^{l+s}\left[G\left(x\left(\bar{\tau}_{j}\right), \bar{\alpha}_{j}\right)-G\left(x\left(\bar{\tau}_{j}\right), \bar{\alpha}_{j-1}\right)\right] \|= \\
& =\| \sum_{j=1}^{l}\left[G\left(x\left(\bar{\tau}_{j}\right), \bar{\alpha}_{j}\right)-G\left(x\left(\bar{\tau}_{j}\right), \bar{\alpha}_{j-1}\right)-G\left(x\left(\tau_{m}\right), \bar{\alpha}_{j}\right)+G\left(x\left(\tau_{m}\right), \bar{\alpha}_{j-1}\right)\right]- \\
& -G\left(x\left(\tau_{m}\right), \tau_{m}+\right)+G\left(x\left(\tau_{m}\right), \alpha_{m-1}\right)-G\left(x\left(\tau_{m}+\right), \alpha_{m}\right)+G\left(x\left(\tau_{m}+\right), \tau_{m}+\right)+ \\
& \sum_{j=l+2}^{l+s}\left[G\left(x\left(\bar{\tau}_{j}\right), \bar{\alpha}_{j}\right)-G\left(x\left(\bar{\tau}_{j}\right), \bar{\alpha}_{j-1}\right)-G\left(x\left(\tau_{m}+\right), \bar{\alpha}_{j}\right)+G\left(x\left(\tau_{m}+\right), \bar{\alpha}_{j-1}\right)\right]+ \\
& +G\left(x\left(\tau_{m}\right), \bar{\alpha}_{l}\right)-G\left(x\left(\tau_{m}\right), \bar{\alpha}_{0}\right)+G\left(x\left(\bar{\tau}_{l+1}\right), \bar{\alpha}_{l+1}\right)-G\left(x\left(\bar{\tau}_{l+1}\right), \bar{\alpha}_{l}\right)+ \\
& +G\left(x\left(\tau_{m}+\right), \bar{\alpha}_{l+s}\right)-G\left(x\left(\tau_{m}+\right), \bar{\alpha}_{l+1}\right) \|= \\
& =\| \sum_{j=1}^{l}\left[G\left(x\left(\bar{\tau}_{j}\right), \bar{\alpha}_{j}\right)-G\left(x\left(\bar{\tau}_{j}\right), \bar{\alpha}_{j-1}\right)-G\left(x\left(\tau_{m}\right), \bar{\alpha}_{j}\right)+G\left(x\left(\tau_{m}\right), \bar{\alpha}_{j-1}\right)\right]- \\
& -G\left(x\left(\tau_{m}\right), \tau_{m}+\right)+G\left(x\left(\tau_{m}\right), \alpha_{m-1}\right)-G\left(x\left(\tau_{m}+\right), \alpha_{m}\right)+G\left(x\left(\tau_{m}+\right), \tau_{m}+\right)+ \\
& +G\left(x\left(\tau_{m}\right), \tau_{m}\right)-G\left(x\left(\tau_{m}\right), \alpha_{m-1}\right)+
\end{aligned}
$$




$$
\begin{aligned}
& +G\left(x\left(\bar{\tau}_{l+1}\right), \bar{\alpha}_{l+1}\right)-G\left(x\left(\bar{\tau}_{l+1}\right), \tau_{m}\right)+G\left(x\left(\tau_{m}+\right), \bar{\alpha}_{l+s}\right)-G\left(x\left(\tau_{m}+\right), \bar{\alpha}_{l+1}\right)+ \\
& +\sum_{j=l+2}^{l+s}\left[G\left(x\left(\bar{\tau}_{j}\right), \bar{\alpha}_{j}\right)-G\left(x\left(\bar{\tau}_{j}\right), \bar{\alpha}_{j-1}\right)-G\left(x\left(\tau_{m}+\right), \bar{\alpha}_{j}\right)+G\left(x\left(\tau_{m}+\right), \bar{\alpha}_{j-1}\right)\right] \|= \\
& =\| \sum_{j=1}^{l}\left[G\left(x\left(\bar{\tau}_{j}\right), \bar{\alpha}_{j}\right)-G\left(x\left(\bar{\tau}_{j}\right), \bar{\alpha}_{j-1}\right)-G\left(x\left(\tau_{m}\right), \bar{\alpha}_{j}\right)+G\left(x\left(\tau_{m}\right), \bar{\alpha}_{j-1}\right)\right]- \\
& -G\left(x\left(\tau_{m}\right), \tau_{m}+\right)+G\left(x\left(\tau_{m}+\right), \tau_{m}+\right)+G\left(x\left(\tau_{m}\right), \tau_{m}\right)+ \\
& +G\left(x\left(\bar{\tau}_{l+1}\right), \bar{\alpha}_{l+1}\right)-G\left(x\left(\bar{\tau}_{l+1}\right), \tau_{m}\right)-G\left(x\left(\tau_{m}+\right), \bar{\alpha}_{l+1}\right)+ \\
& +\sum_{j=l+2}^{l+s}\left[G\left(x\left(\bar{\tau}_{j}\right), \bar{\alpha}_{j}\right)-G\left(x\left(\bar{\tau}_{j}\right), \operatorname{bar}_{j-1}\right)-G\left(x\left(\tau_{m}+\right), \bar{\alpha}_{j}\right)+G\left(x\left(\tau_{m}+\right), \bar{\alpha}_{j-1}\right)\right] \|= \\
& =\| \sum_{j=1}^{l}\left[G\left(x\left(\bar{\tau}_{j}\right), \bar{\alpha}_{j}\right)-G\left(x\left(\bar{\tau}_{j}\right), \bar{\alpha}_{j-1}\right)-G\left(x\left(\tau_{m}\right), \bar{\alpha}_{j}\right)+G\left(x\left(\tau_{m}\right), \bar{\alpha}_{j-1}\right)\right]- \\
& -G\left(x\left(\tau_{m}\right), \tau_{m}+\right)+G\left(x\left(\tau_{m}\right), \bar{\alpha}_{l}\right)+G\left(x\left(\bar{\tau}_{l+1}\right), \bar{\alpha}_{l+1}\right)- \\
& -G\left(x\left(\bar{\tau}_{l+1}\right), \bar{\alpha}_{l}\right)-G\left(x\left(\tau_{m}+\right), \bar{\alpha}_{l+1}\right)+G\left(x\left(\tau_{m}+\right), \tau_{m}+\right)+ \\
& +\sum_{j=l+2}^{l+s}\left[G\left(x\left(\bar{\tau}_{j}\right), \bar{\alpha}_{j}\right)-G\left(x\left(\bar{\tau}_{j}\right), \bar{\alpha}_{j-1}\right)--G\left(x\left(\tau_{m}+\right), \bar{\alpha}_{j}\right)+G\left(x\left(\tau_{m}+\right), \bar{\alpha}_{j-1}\right)\right] \| \leq \\
& \leq \sum_{j=1}^{l} \|\left[G\left(x\left(\bar{\tau}_{j}\right), \bar{\alpha}_{j}\right)-G\left(x\left(\bar{\tau}_{j}\right), \bar{\alpha}_{j-1}\right)-G\left(x\left(\tau_{m}\right), \bar{\alpha}_{j}\right)+\right. \\
& \left.+G\left(x\left(\tau_{m}\right), \bar{\alpha}_{j-1}\right)\right]\|+\| G\left(x\left(\tau_{m}\right), \bar{\alpha}_{l}\right)+G\left(x\left(\bar{\tau}_{l+1}\right), \bar{\alpha}_{l+1}\right)-G\left(x\left(\bar{\tau}_{l+1}\right), \bar{\alpha}_{l}\right)- \\
& -G\left(x\left(\tau_{m}\right), \tau_{m}+\right)-G\left(x\left(\tau_{m}+\right), \bar{\alpha}_{l+1}\right)+G\left(x\left(\tau_{m}+\right), \tau_{m}+\right) \|+ \\
& \sum_{j=l+2}^{l+s}\left\|\left[G\left(x\left(\bar{\tau}_{j}\right), \bar{\alpha}_{j}\right)-G\left(x\left(\bar{\tau}_{j}\right), \bar{\alpha}_{j-1}\right)-G\left(x\left(\tau_{m}+\right), \bar{\alpha}_{j}\right)+G\left(x\left(\tau_{m}+\right), \bar{\alpha}_{j-1}\right)\right]\right\| \\
& \leq \sum_{j=1}^{l}\left\|\left[G\left(x\left(\bar{\tau}_{j}\right), \bar{\alpha}_{j}\right)-G\left(x\left(\bar{\tau}_{j}\right), \bar{\alpha}_{j-1}\right)-G\left(x\left(\tau_{m}\right), \bar{\alpha}_{j}\right)+G\left(x\left(\tau_{m}\right), \bar{\alpha}_{j-1}\right)\right]\right\|+ \\
& +\| G\left(x\left(\tau_{m}\right), \tau_{m}\right)+G\left(x\left(\tau_{m}\right), \bar{\alpha}_{l+1}\right)-G\left(x\left(\tau_{m}\right), \tau_{m}\right)-G\left(x\left(\tau_{m}\right), \tau_{m}+\right)- \\
& -G\left(x\left(\tau_{m}+\right), \bar{\alpha}_{l+1}\right)+G\left(x\left(\tau_{m}+\right), \tau_{m}+\right) \|+ \\
& +\sum_{j=l+2}^{l+s}\left\|\left[G\left(x\left(\bar{\tau}_{j}\right), \bar{\alpha}_{j}\right)-G\left(x\left(\bar{\tau}_{j}\right), \bar{\alpha}_{j-1}\right)-G\left(x\left(\tau_{m}+\right), \bar{\alpha}_{j}\right)+G\left(x\left(\tau_{m}+\right), \bar{\alpha}_{j-1}\right)\right]\right\| \leq
\end{aligned}
$$




$$
\begin{gathered}
\leq \sum_{j=1}^{l}\left\|\left[G\left(x\left(\bar{\tau}_{j}\right), \bar{\alpha}_{j}\right)-G\left(x\left(\bar{\tau}_{j}\right), \bar{\alpha}_{j-1}\right)-G\left(x\left(\tau_{m}\right), \bar{\alpha}_{j}\right)+G\left(x\left(\tau_{m}\right), \bar{\alpha}_{j-1}\right)\right]\right\|+ \\
+\left\|G\left(x\left(\tau_{m}\right), \bar{\alpha}_{l+1}\right)-G\left(x\left(\tau_{m}\right), \tau_{m}+\right)-G\left(x\left(\tau_{m}+\right), \bar{\alpha}_{l+1}\right)+G\left(x\left(\tau_{m}+\right), \tau_{m}+\right)\right\|+ \\
+\sum_{j=l+2}^{l+s}\left\|\left[G\left(x\left(\bar{\tau}_{j}\right), \bar{\alpha}_{j}\right)-G\left(x\left(\bar{\tau}_{j}\right), \bar{\alpha}_{j-1}\right)-G\left(x\left(\tau_{m}+\right), \bar{\alpha}_{j}\right)+G\left(x(\tau+), \bar{\alpha}_{j-1}\right)\right]\right\| \\
\leq \sum_{j=1}^{l}\left\|x\left(\bar{\tau}_{j}\right)-x\left(\tau_{m}\right)\right\|\left(h\left(\bar{\alpha}_{j}\right)-h\left(\bar{\alpha}_{j-1}\right)\right)+\left\|x\left(\tau_{m}+\right)-x\left(\tau_{m}\right)\right\|\left(h\left(\bar{\alpha}_{l+1}\right)-h\left(\tau_{m}+\right)\right)+ \\
+\sum_{j=l+2}^{l+s}\left\|x\left(\bar{\tau}_{j}\right)-x\left(\tau_{m}+\right)\right\|\left(h\left(\bar{\alpha}_{j}\right)-h\left(\bar{\alpha}_{j-1}\right)\right) \leq \\
\leq \sum_{j=1}^{l}\left(h_{0}\left(\bar{\tau}_{j}\right)-h_{0}\left(\tau_{m}\right)\right)\left(h\left(\bar{\alpha}_{j}\right)-h\left(\bar{\alpha}_{j-1}\right)\right)+\left(h_{0}\left(\tau_{m}+\right)-h_{0}\left(\tau_{m}\right)\right)\left(h\left(\bar{\alpha}_{l+1}\right)-h\left(\tau_{m}+\right)\right)+ \\
+\sum_{j=l+2}^{l+s}\left(h_{0}\left(\bar{\tau}_{j}\right)-h_{0}\left(\tau_{m}+\right)\right)\left(h\left(\bar{\alpha}_{j}\right)-h\left(\bar{\alpha}_{j-1}\right)\right) \\
\leq \eta\left(h\left(\alpha_{m}\right)-h\left(\alpha_{m-1}\right)+h_{0}\left(\alpha_{m}\right)-h_{0}\left(\alpha_{m-1}\right)\right) . \\
\leq \eta\left(h\left(\alpha_{m}\right)-h\left(\alpha_{m-1}\right)+h_{0}\left(\tau_{m}+\right)-h_{0}\left(\tau_{m}\right)\right) \\
\leq \eta\left(h\left(\tau_{m}\right)-\right. \\
h\left(\alpha_{m-1}\right)+\left(h_{0}\left(\tau_{m}+\right)-h_{0}\left(\tau_{m}\right)\right) \eta+\eta\left(h\left(\alpha_{m}\right)-h\left(\bar{\alpha}_{l+1}\right) \leq\right. \\
\leq
\end{gathered}
$$

A desigualdade acima junto com (1.39) implicam a desigualdade

$$
\begin{gathered}
\left\|\int_{\alpha_{m-1}}^{\alpha_{m}} D G(x(\tau), t)-S_{m}\right\| \leq \\
\leq\left\|\int_{\alpha_{m-1}}^{\alpha_{m}} D G(x(\tau), t)-S\left(A^{+}\right)-S\left(A^{-}\right)\right\|+\left\|S\left(A^{+}\right)-S\left(A^{-}\right)-S_{m}\right\| \\
\leq \varepsilon+\left\|S\left(A^{-}\right)+S\left(A^{+}\right)-S_{m}\right\|<\varepsilon+\eta\left(h\left(\alpha_{m}\right)-h\left(\alpha_{m-1}\right)+h_{0}\left(\alpha_{m}\right)-h_{0}\left(\alpha_{m-1}\right)\right) .
\end{gathered}
$$

Como $\varepsilon>0$ pode ser escolhido arbitrariamente pequeno, temos

$$
\left\|\int_{\alpha_{m-1}}^{\alpha_{m}} D G(x(\tau), t)-S_{m}\right\| \leq \eta\left(h\left(\alpha_{m}\right)-h\left(\alpha_{m-1}\right)+h_{0}\left(\alpha_{m}\right)-h_{0}\left(\alpha_{m-1}\right)\right) .
$$

Para o caso em que $\tau \notin D$, a mesma desigualdade acima pode ser provada de modo 
análogo. Daí, usando (1.40) e a desigualdade abaixo

$$
\left\|\int_{\alpha}^{\beta} D G(x(\tau), t)-\sum_{m=1}^{k} S_{m}\right\| \leq \sum_{m=1}^{k}\left\|\int_{\alpha_{m-1}}^{\alpha_{m}} D G(x(\tau), t)-S_{m}\right\|,
$$

obtemos

$$
\begin{gathered}
\left\|\int_{\alpha}^{\beta} D G(x(\tau), t)-\sum_{m=1}^{k} S_{m}\right\|=\left\|\sum_{m=1}^{k} \int_{\alpha_{m-1}}^{\alpha_{m}} D G(x(\tau), t)-\sum_{m=1}^{k} S_{m}\right\| \\
\leq \sum_{m=1}^{k}\left\|\int_{\alpha_{m-1}}^{\alpha_{m}} D G(x(\tau), t)-S_{m}\right\| \leq \\
\leq \sum_{m=1}^{k} \eta\left(h\left(\alpha_{m}\right)-h\left(\alpha_{m-1}\right)+h_{0}\left(\alpha_{m}\right)-h_{0}\left(\alpha_{m-1}\right)\right)=\eta\left(h(\beta)-h(\alpha)+h_{0}(\beta)-h_{0}(\alpha)\right)
\end{gathered}
$$

e temos o resultado desejado. 


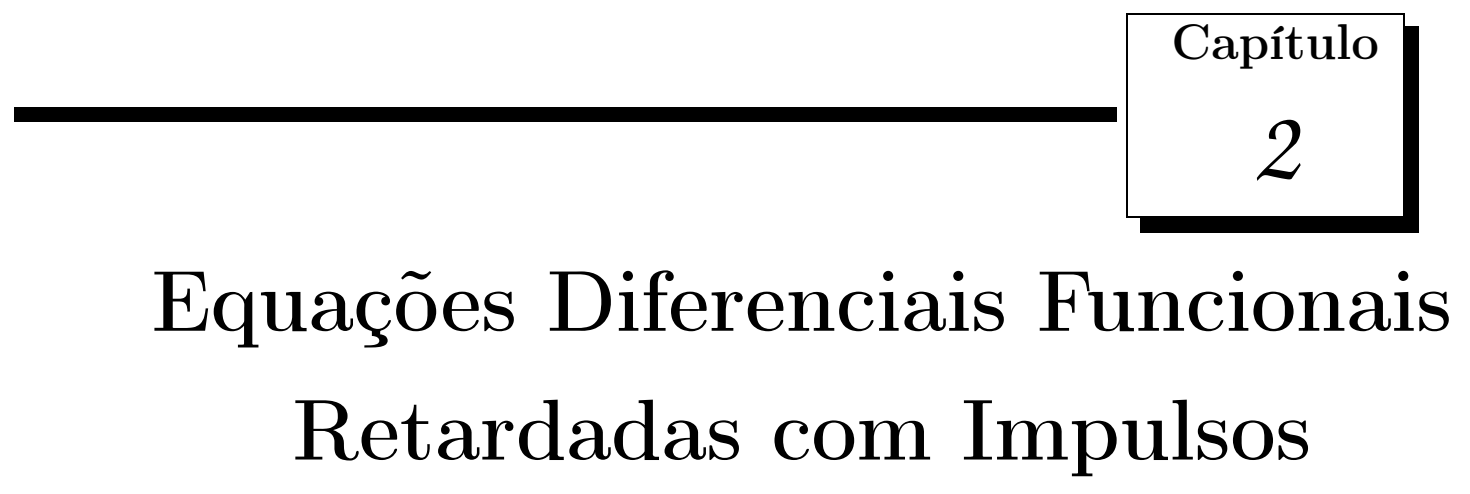

Neste capítulo, iremos introduzir algumas definições básicas e alguns resultados sobre equações diferenciais funcionais retardadas com impulsos (EDFRIs), que serão de fundamental importância ao longo deste trabalho.

Este capítulo está dividido em três seções. Na primeira seção, faremos uma abordagem sobre as equações diferenciais funcionais com retardos (EDFRs), trazendo algumas definições básicas e resultados essenciais para o nosso trabalho. A principal referência para esta parte será [13]. Na segunda seção, trataremos da teoria de equações diferenciais impulsivas (EDIs), quando descreveremos vários tipos de sistemas de equações diferenciais impulsivas, exibindo exemplos. A principal referência para esta parte será [21]. Por fim, na terceira seção, trataremos da teoria de equações diferenciais funcionais retardadas com impulsos (EDFRIs), onde relacionaremos a teoria de EDFRs com a teoria de EDIs. A principal referência para esta parte será [10].

Omitiremos algumas demonstrações dos resultados apresentados, quando considerarmos desnecessárias.

\subsection{Noções básicas de EDFRs}

Nesta seção, consideraremos $a, b$ como sendo números reais, $n$ como sendo um inteiro maior ou igual a $1, \mathbb{R}^{n}$ como sendo o espaço vetorial $n$-dimensional sobre os reais com norma denotada por $\|\cdot\|$ e $C\left([a, b], \mathbb{R}^{n}\right)$ como sendo o espaço de Banach das funções contínuas de $[a, b]$ em $\mathbb{R}^{n}$ com a topologia da convergência uniforme. Supondo $r>0$ e 
tomando $[a, b]=[-r, 0]$, denotaremos por $C=C\left([-r, 0], \mathbb{R}^{n}\right)$ o espaço de Banach das funções contínuas de $[-r, 0]$ em $\mathbb{R}^{n}$ com a norma

$$
\|\varphi\|=\sup _{-r \leq \theta \leq 0}\|\varphi(\theta)\|
$$

Seja $V \subseteq \mathbb{R} \times C$ um aberto, então $C\left(V, \mathbb{R}^{n}\right)$ será o espaço das funções contínuas de $V$ em $\mathbb{R}^{n}$. Denotaremos por $C^{0}\left(V, \mathbb{R}^{n}\right)$ o espaço das funções contínuas e limitadas de $V$ em $\mathbb{R}^{n}$. Este espaço se tornará um espaço de Banach, se considerarmos sua norma como sendo a norma induzida de $C\left(V, \mathbb{R}^{n}\right)$.

Dados $\sigma \in \mathbb{R}, A>0$ e $x \in C\left([\sigma-r, \sigma+A), \mathbb{R}^{n}\right)$, então para cada $t \in[\sigma, \sigma+A)$, definimos $x_{t} \in C\left([-r, 0], \mathbb{R}^{n}\right)$ como sendo

$$
x_{t}(\theta)=x(t+\theta), \quad-r \leq \theta \leq 0 .
$$

Definição 2.1. Se $\Omega \subseteq \mathbb{R} \times C$ for um aberto e $f: \Omega \rightarrow \mathbb{R}^{n}$ for uma função, então diremos que a equação

$$
\dot{x}(t)=f\left(t, x_{t}\right), \quad\left(\dot{x}=\frac{d x}{d t}\right),
$$

será uma equação diferencial funcional retardada sobre $\Omega$.

Às vezes, usaremos a notação $\operatorname{EDFR}(f)$ para nos referirmos a uma equação do tipo (2.1). Definiremos, a seguir, o conceito de solução da equação (2.1).

Definição 2.2. Uma função $x$ será dita uma solução da equação (2.1) em $[\sigma-r, \sigma+A)$ se existirem $\sigma \in \mathbb{R}$ e $A>0$ tais que $x \in C\left([\sigma-r, \sigma+A), \mathbb{R}^{n}\right),\left(t, x_{t}\right) \in \Omega$ e $x(t)$ satisfizer a equação (2.1), para $t \in[\sigma, \sigma+A)$.

Temos também a definição de uma solução de (2.1) com valor inicial, que apresentamos a seguir.

Definição 2.3. Dados $\sigma \in \mathbb{R}, \phi \in C$, diremos que $x(\sigma, \phi, f)$ será uma solução de (2.1) com valor inicial $\phi$ em $\sigma$, ou simplesmente que será uma solução passando por $(\sigma, \phi)$, se existir um $A>0$ tal que $x(\sigma, \phi, f)$ é uma solução de $(2.1)$ em $[\sigma-r, \sigma+A)$ e $x_{\sigma}(\sigma, \phi, f)=$ $\phi$.

Com estas notações e terminologias, podemos enunciar alguns resultados básicos que podem ser encontrados em [13].

Lema 2.4 ([13], Lema 2.1). Se $x \in C\left([\sigma-r, \sigma+\alpha], \mathbb{R}^{n}\right)$, então $x_{t}$ será uma função contínua como função de $t$ para $t \in[\sigma, \sigma+\alpha]$. 
Prova: Como $x \in C\left([\sigma-r, \sigma+\alpha], \mathbb{R}^{n}\right)$, então $x$ é contínua em $[\sigma-r, \sigma+\alpha]$. E como $[\sigma-r, \sigma+\alpha]$ é compacto, $x$ é uniformemente contínua. Portanto, para todo $\varepsilon>0$, existe um $\delta>0$ tal que $|x(t)-x(\tau)|<\varepsilon$, se $|t-\tau|<\delta$. De fato, se $|t-\tau|<\delta$ e $t \in[\sigma, \sigma+\alpha]$, então

$$
|x(t+\theta)-x(\tau+\theta)|<\varepsilon
$$

para todo $\theta \in[-r, 0]$. Isto prova o lema.

Lema 2.5 ([13], Lema 1.1). Se $\sigma \in \mathbb{R}$ e $\phi \in C$ forem dados e $f(t, \phi)$ for contínua, então encontrar uma solução da equação (2.1) passando por $(\sigma, \phi)$ será equivalente a resolver a equação integral

$$
\begin{gathered}
x_{\sigma}=\phi \\
x(t)=\phi(0)+\int_{\sigma}^{t} f\left(s, x_{s}\right) d s, \quad t \geq \sigma
\end{gathered}
$$

Prova: A demonstração deste lema segue imediatamente do lema anterior e do teorema fundamental do cálculo.

O próximo resultado aborda a existência de uma solução de uma dada EDFR com valor inicial.

Teorema 2.6 (Existência local - [13], Teorema 2.1). Suponhamos que $\Omega$ seja um subconjunto aberto de $\mathbb{R} \times C$ e $f \in C\left(\Omega, \mathbb{R}^{n}\right)$. Se $(\sigma, \varphi) \in \Omega$, então existirá uma solução local de (2.1) por $(\sigma, \varphi)$. Mais geralmente, se $W \subseteq \Omega$ for compacto e $f \in C\left(\Omega, \mathbb{R}^{n}\right)$, então existirá uma vizinhança $V \subseteq \Omega$ de $W$ tal que $f \in C^{0}\left(V, \mathbb{R}^{n}\right)$, e existirão uma vizinhança $U \subseteq C^{0}\left(V, \mathbb{R}^{n}\right)$ de $f$ e um $\alpha>0$ tais que, para $(\sigma, \varphi) \in W$ e $g \in U$, existirá uma solução $x(\sigma, \varphi, g)$ de (2.1) passando por $(\sigma, \varphi)$ em $[\sigma-r, \sigma+\alpha]$.

Omitiremos a demonstração do Teorema 2.6, devido à sua extensão. Para o leitor interessado, ela pode ser encontrada em [13], página 43.

A seguir, enunciaremos um teorema que garante a existência de uma única solução local de (2.1) passando por $(\sigma, \varphi)$.

Teorema 2.7 (Unicidade - [13], Teorema 2.3). Se $\Omega \subseteq \mathbb{R} \times C$ for um aberto, $(\sigma, \varphi) \in \Omega$, $f: \Omega \rightarrow \mathbb{R}^{n}$ for contínua e $f(t, \varphi)$ for localmente lipschtiziana com relação a $\varphi$ em $\Omega$. Então existirá uma única solução de (2.1) passando por $(\sigma, \varphi)$.

Prova: Definamos $I_{\alpha}=[0, \alpha]$ e $B_{\beta}=\{\varphi \in C:|\varphi| \leq \beta\}$. Suponhamos que $x$ e $y$ sejam soluções da equação (2.1) em $[\sigma-r, \sigma+\alpha] \operatorname{com} x_{\sigma}=\varphi=y_{\sigma}$. Então, pelo Lema 2.5 , temos

$$
x_{\sigma}=\varphi
$$




$$
x(t)=\varphi(0)+\int_{\sigma}^{t} f\left(s, x_{s}\right) d s, \quad t \geq \sigma
$$

e

$$
\begin{gathered}
y_{\sigma}=\varphi \\
y(t)=\varphi(0)+\int_{\sigma}^{t} f\left(s, y_{s}\right) d s, \quad t \geq \sigma
\end{gathered}
$$

Destas relações, temos a relação abaixo

$$
\begin{gathered}
x_{\sigma}=y_{\sigma}=\varphi \\
x(t)-y(t)=\int_{\sigma}^{t}\left[f\left(s, x_{s}\right)-f\left(s, y_{s}\right)\right] d s, \quad t \geq \sigma
\end{gathered}
$$

Se $k$ for a constante de Lipschitz de $f(t, \varphi)$ em algum conjunto compacto contendo as trajetórias $\left\{\left(t, x_{t}\right)\right\}$ e $\left\{\left(t, y_{t}\right)\right\}, t \in I_{\alpha}$, então poderemos escolher $\bar{\alpha}$ de modo que $k \bar{\alpha}<1$. Assim, para $t \in I_{\bar{\alpha}}$, teremos

$$
\begin{gathered}
|x(t)-y(t)| \leq\left|\int_{\sigma}^{t} f\left(s, x_{s}\right)-f\left(s, y_{s}\right) d s\right| \leq \int_{\sigma}^{t}\left|f\left(s, x_{s}\right)-f\left(s, y_{s}\right)\right| d s \leq \\
\leq \int_{\sigma}^{t} k\left|y_{s}-x_{s}\right| d s \leq k \sup _{\sigma \leq s \leq t}\left|y_{s}-x_{s}\right| \int_{\sigma}^{t} d s \leq k \sup _{\sigma \leq s \leq t}\left|y_{s}-x_{s}\right| \bar{\alpha}
\end{gathered}
$$

e isto implicará que $x(t)=y(t)$, para $t \in I_{\bar{\alpha}}$. Se repetirmos este processo, tomando os intervalos de comprimento $\bar{\alpha}$ cada vez maiores, teremos o resultado desejado.

O próximo teorema trata da dependência contínua de soluções de certa EDFR com respeito aos valores iniciais.

Teorema 2.8 (Dependência contínua - [13], Teorema 2.2). Suponhamos que $\Omega \subseteq \mathbb{R} \times C$ seja aberto, $\left(\sigma^{0}, \varphi^{0}\right) \in \Omega, f^{0} \in C\left(\Omega, \mathbb{R}^{n}\right)$ e $x^{0}$ seja uma solução de $\operatorname{EDFR}\left(f^{0}\right)$, por $\left(\sigma^{0}, \varphi^{0}\right)$, a qual existe e é única sobre $\left[\sigma^{0}-r, b\right]$. Sejam $W^{0} \subseteq \Omega$ o conjunto compacto definido por $W^{0}=\left\{\left(t,\left(x^{0}\right)_{t}\right) ; t \in\left[\sigma^{0}, b\right]\right\}$ e $V^{0}$ uma vizinhança de $W^{0}$ sobre a qual $f^{0}$ é limitada. Se $\left(\sigma^{k}, \varphi^{k}, f^{k}\right), k=1,2, \ldots$, satisfizer $\sigma^{k} \rightarrow \sigma^{0}, \varphi^{k} \rightarrow \varphi^{0} e\left|f^{k}-f^{0}\right| \rightarrow 0$ quando $k \rightarrow \infty$, então existirá um $k^{0} \in \mathbb{N}$ tal que, para $k \geq k^{0}$, a $\operatorname{EDFR}\left(f^{k}\right)$ será tal que cada solução $x^{k}=x^{k}\left(\sigma^{k}, \varphi^{k}, f^{k}\right)$ passando por $\left(\sigma^{k}, \varphi^{k}\right)$ existirá sobre $\left[\sigma^{k}-r, b\right]$ e $x^{k} \rightarrow x^{0}$ uniformemente sobre $\left[\sigma^{0}-r, b\right]$.

Observação 2.9. No teorema acima, como pode ocorrer de nem todas as $x^{k}$ estarem definidas em $\left[\sigma^{0}-r, b\right]$, quando escrevermos $x^{k} \rightarrow x^{0}$ uniformemente sobre $\left[\sigma^{0}-r, b\right]$, entenderemos que, para qualquer $\varepsilon>0$, existirá um $k_{1}(\varepsilon) \in \mathbb{N}$ tal que para $k \geq k_{1}(\varepsilon)$, $x^{k}(t)$ estará definida sobre $\left[\sigma^{0}-r+\varepsilon, b\right]$ e $x^{k} \rightarrow x^{0}$ uniformemente sobre $\left[\sigma^{0}-r+\varepsilon, b\right]$. 
Novamente, não incluiremos a demonsração deste resultado, devido à sua extensão. Entretanto ela pode ser encontrada em [13], páginas 43 e 44.

Para as definições abaixo, vamos supor que $f$ na Definição 2.1 seja uma função contínua.

Definição 2.10. Se $x$ for uma solução de (2.1) em um intervalo $[\sigma, a), a>\sigma$, diremos que $\widetilde{x}$ será uma continuação de $x$, se existir um $b>$ a tal que $\widetilde{x}$ está definida em $[\sigma-r, b)$ e $\widetilde{x}$ coincidir com $x$ em $[\sigma-r, a)$ e satisfizer a equação (2.1) em $[\sigma, b)$. Uma solução $x$ será não-continuável, se tal continuação não existir, ou seja, se o intervalo $[\sigma, a)$ for o intervalo maximal de existência da solução $x$.

Com estas definições e com os resultados apresentados anteriormente, podemos enunciar alguns resultados de grande importância sobre continuação de soluções. Não apresentaremos as demonstrações destes resultados, tendo em vista a extensão destas. Entretanto, elas podem ser encontradas em [13], páginas 45 e 46.

Os resultados seguintes tratam de não-continuação de soluções.

Teorema 2.11 ([13], Teorema 3.1). Suponhamos que $\Omega$ seja um conjunto aberto de $\mathbb{R} \times C$ e que $f \in C\left(\Omega, \mathbb{R}^{n}\right)$. Se $x$ for uma solução não continuável da equação $(2.1)$ em $[\sigma-r, b)$, então, para todo conjunto compacto $W$ em $\Omega$, existirá um $t_{W}$ tal que $\left(t, x_{t}\right) \notin W$ para $t_{W} \leq t<b$.

Corolário 2.12 ([13], Corolário 3.1). Suponhamos que $\Omega$ seja um conjunto aberto de $\mathbb{R} \times C$ e que $f \in C\left(\Omega, \mathbb{R}^{n}\right)$. Se $x$ for uma solução não continuável da equação (2.1) em $[\sigma-r, b)$ e $W$ for o fecho do conjunto $\left\{\left(t, x_{t}\right): \sigma \leq t<b\right\}$ em $\mathbb{R} \times C$, então se $W$ for compacto, existirá uma sequência de números reais $\left\{t_{k}\right\}$, com $t_{k} \rightarrow b-$ quando $k \rightarrow \infty$, tal que $\left(t_{k}, x_{t_{k}}\right)$ tende a $\partial \Omega$ quando $k \rightarrow \infty$, onde $\partial \Omega$ denota a fronteira de $\Omega$. Se $r>0$, então existirá um $\varphi \in C$ tal que $(b, \varphi) \in \partial \Omega e\left(t, x_{t}\right) \rightarrow(b, \varphi)$ quando $t \rightarrow b^{-}$.

Teorema 2.13 ([13], Teorema 3.2). Suponhamos que $\Omega$ seja um conjunto aberto de $\mathbb{R} \times C$ e que $f: \Omega \mapsto \mathbb{R}^{n}$ seja completamente continua, ou seja, $f$ é continua e leva conjuntos fechados e limitados de $\Omega$ em conjuntos limitados de $\mathbb{R}^{n}$. Suponhamos, também, que $x$ seja uma solução não continuável da equação (2.1) em $[\sigma-r, b)$. Então, para todo conjunto limitado e fechado $U$ em $\mathbb{R} \times C, U$ em $\Omega$, existirá um $t_{U}$ tal que $\left(t, x_{t}\right) \notin U$ para $t_{U} \leq t<b$.

\subsection{Noções básicas de EDIs}

Inciaremos a fundamentação teórica sobre equações diferenciais funcionais retardadas com impulsos, listando e esclarecendo alguns aspectos básicos sobre a teoria das equações diferenciais impulsivas sem retardo que trataremos, abreviadamente, por EDIs. 
Uma EDI (equação diferencial impulsiva) ou sistema impulsivo pode ser representada da forma seguinte. Considera-se

i) uma equação diferencial

$$
\dot{x}=f(t, x) \quad\left(\dot{x}=\frac{d x}{d t}\right)
$$

onde $f: \mathbb{R}_{+} \times \Omega \rightarrow \mathbb{R}^{n}$ e $\Omega$ é um subconjunto aberto do $\mathbb{R}^{n}, \mathbb{R}^{n}$ é o espaço euclidiano n-dimensional e $\mathbb{R}_{+}$é a reta real não negativa;

ii) subconjuntos $M(t), N(t) \subset \Omega, t \in \mathbb{R}_{+}$;

iii) funções $A(t): M(t) \rightarrow N(t), t \in \mathbb{R}_{+}$.

Descreveremos, a seguir, o comportamento do processo de evolução de i), ii) e iii) acima.

Seja $x(t)=x\left(t ; t_{0}, x_{0}\right)$ uma solução do sistema (2.2) passando pelo ponto $\left(t_{0}, x_{0}\right) \in$ $\mathbb{R}_{+} \times \Omega$, que denotaremos po $P_{t_{0}}$. O ponto $P_{t}=(t, x(t))$ inicia seu movimento no ponto inicial $P_{t_{0}}=\left(t_{0}, x_{0}\right)$ e move-se ao longo da curva $\left\{(t, x): t \geq t_{0}, x=x(t)\right\}$ até o tempo $t_{1}>t_{0}$ no qual $P_{t}$ encontra o conjunto $(t, M(t))$. Em $t=t_{1}$, o operador $A(t)$ transfere o ponto $P_{t_{1}}=\left(t_{1}, x\left(t_{1}\right)\right)$ para o ponto $P_{t_{1}^{+}}=\left(t_{1}, x_{1}^{+}\right) \in\left(t_{1}, N\left(t_{1}\right)\right)$, onde $x_{1}^{+}=A\left(t_{1}\right) x\left(t_{1}\right)$. Deste modo, o ponto $P_{t}$ continua seu movimento ao longo da curva $x(t)=x\left(t ; t_{1}, x_{1}^{+}\right)$, que é solução de (2.2) com condição inicial $P_{t_{1}^{+}}=\left(t_{1}, x_{1}^{+}\right)$, até encontrar novamente o conjunto $(t, M(t))$ num tempo $t_{2}>t_{1}$. Em seguida, o ponto $P_{t_{2}}=\left(t_{2}, x\left(t_{2}\right)\right)$ é transferido pelo operador $A(t)$ para o ponto $P_{t_{2}^{+}}=\left(t_{2}, x_{2}^{+}\right) \in\left(t_{2}, N\left(t_{2}\right)\right)$, onde $x_{2}^{+}=A\left(t_{2}\right) x\left(t_{2}\right)$. Agora, o movimento de $P_{t}$ se inicia em $\left(t_{2}, x_{2}^{+}\right)$ao longo da solução $x(t)=x\left(t ; t_{2}, x_{2}^{+}\right)$de $(2.2)$ e o processo continua ao longo da solução de (2.2), caso esta exista, repetindo o procedimento descrito acima.

Definição 2.14. A curva descrita por $P_{t}$ acima é chamada curva integral e a função que define esta curva é a solução da equação diferencial impulsiva.

Note que uma solução de uma equação diferencial impulsiva como acima poderá ser:

a) uma função contínua, se a curva integral $\left\{(t, x): t \geq t_{0}, x=x(t)\right\}$ não interceptar o conjunto $M(t)$, ou se ela atingir $M(t)$ somente nos pontos fixos do operador $A(t)$;

b) uma função contínua por partes com um número finito de descontinuidades de primeira espécie, se a curva integral encontrar $M(t)$ em um número finito de pontos que não sejam pontos fixos do operador $A(t)$; 
c) uma função contínua por partes com uma quantidade enumerável de descontinuidades de primeira espécie, se a curva integral encontrar o conjunto $M(t)$ em uma quantidade enumerável de pontos que não sejam pontos fixos do operador $A(t)$.

Chamaremos os instantes $t=t_{k}, k=1,2, \ldots$, nos quais a curva integral atinge $M(t)$ de momentos de impulso. Iremos supor que qualquer solução $x(t)$ da EDI (2.2) seja contínua à esquerda em $t_{k}$, para $k=1,2,3, \ldots$, isto é, $x\left(t_{k}^{-}\right):=\lim _{h \rightarrow 0^{+}} x\left(t_{k}-h\right)=x\left(t_{k}\right)$.

A seguir, analisaremos alguns tipos de sistemas diferenciais com impulsos.

Começaremos analisando os sistemas com impulsos em tempos fixados. Estes sistemas são equações diferenciais impulsivas cujos momentos de impulsos são pré estabelecidos, ou seja, os impulsos ocorrem em tempos conhecidos de antemão. Neste sistema, $M(t)$ representa uma seqüência de planos $t=t_{k}$, onde $\left\{t_{k}\right\}$ é uma seqüência de tempos, com $t_{k} \rightarrow+\infty$ quando $k \rightarrow+\infty$.

Definiremos o operador $A(t)$, para $t=t_{k}, k=1,2, \ldots$, da maneira descrita a seguir. A seqüência $\left\{A\left(t_{k}\right)\right\}$ de operadores $A\left(t_{k}\right): \Omega \rightarrow \Omega$ será dada por

$$
x \longmapsto A\left(t_{k}\right) x=x+I_{k}(x),
$$

onde $I_{k}: \Omega \rightarrow \Omega$. O conjunto $N(t)$ será definido para $t=t_{k}$ como sendo $N\left(t_{k}\right)=$ $A\left(t_{k}\right) M\left(t_{k}\right)$, onde $k=1,2, \ldots$

Com a escolha de $M\left(t_{k}\right), N\left(t_{k}\right)$ e $A\left(t_{k}\right), k=1,2, \ldots$, podemos descrever um modelo matemático de um sistema diferencial impulsivo simples em que cada impulso ocorre em tempos fixados da maneira abaixo:

$$
\begin{cases}\dot{x}(t)=f(t, x(t)), & t \neq t_{k}, \\ \Delta x=I_{k}(x), & t=t_{k}, \quad k=1,2, \ldots\end{cases}
$$

onde, para cada $t=t_{k}, \Delta x\left(t_{k}\right)=x\left(t_{k}^{+}\right)-x\left(t_{k}^{-}\right)$e $x\left(t_{k}^{+}\right)=\lim _{h \rightarrow 0^{+}} x\left(t_{k}+h\right)$. Mas como $x$ é contínua à esquerda, $\Delta x\left(t_{k}\right)=x\left(t_{k}^{+}\right)-x\left(t_{k}\right)$. Assim, qualquer solução $x(t)$ de $(2.3)$ satisfaz:

i) $\dot{x}(t)=f(t, x(t)), t \in\left[t_{0}, t_{1}\right], t \in\left(t_{k}, t_{k+1}\right], k=1,2, \ldots$

ii) $\Delta x\left(t_{k}\right)=I_{k}\left(x\left(t_{k}\right)\right), t=t_{k}, k=1,2, \ldots$

Sabemos que os efeitos impulsivos influenciam o comportamento das soluções de (2.3). A continuidade das soluções pode ser afetada pela ação impulsiva, como mostram os exemplos abaixo. 
Exemplo 2.15 ([21], Exemplo 1.1.1). Consideremos a equação diferencial impulsiva

$$
\begin{cases}\dot{x}=0, & t \neq k, \\ \Delta x=\frac{1}{x-1}, & t=k, \quad k=1,2, \ldots\end{cases}
$$

A solução $x(t)$ da equação diferencial ordinária $\dot{x}=0$ é contínua para todo $t$, pois é constante para todo $t$. Note que a solução do sistema (2.4), com condição inicial $x(0)=1$, está definida somente para $0 \leq t \leq 1$, já que a função $I_{k}(x)=\frac{1}{x-1}$ não está definida para $x=1$.

Exemplo 2.16 ([21], Exemplo 1.1.2). Consideremos a equação diferencial impulsiva

$$
\left\{\begin{array}{l}
\dot{x}=1+x^{2}, \quad t \neq \frac{k \pi}{4}, \\
\Delta x=-1, \quad t=\frac{k \pi}{4}, \quad k=1,2, \ldots
\end{array}\right.
$$

Neste caso, a solução $x(t)$ do problema de valor inicial (escrevemos abreviadamente PVI) $\dot{x}=1+x^{2}, x(0)=0$, é contínua no intervalo $\left[0, \frac{\pi}{2}\right)$, pois a solução do PVI é dada por $x(t)=\tan \left(t-\frac{\pi}{2}\right)$. Por outro lado, a solução do sistema (2.5) com condição inicial $x(0)=0$ é dada por

$$
x(t)=\tan \left(t-\frac{k \pi}{4}\right), \quad t \in\left(\frac{k \pi}{4}, \frac{(k+1) \pi}{4}\right] .
$$

Tal solução é periódica de período $\frac{\pi}{4}$ e tem descontinuidades de primeira espécie em $t=\frac{k \pi}{4}$, para $k=1,2, \ldots$ A Figura 2.1 ilustra este fato.

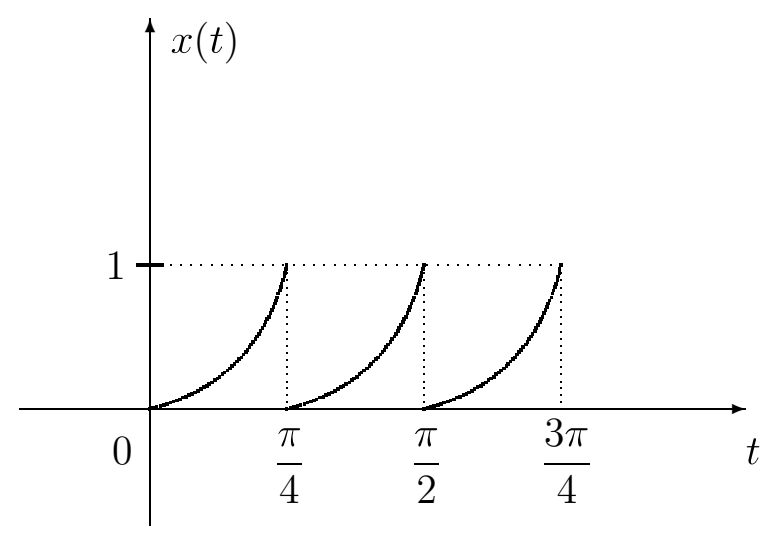

Figura 2.1: Curva integral do sistema impulsivo (2.5), com condição inicial $x(0)=0$. 
Agora, iremos analisar os sistemas com impulsos em tempos variáveis. Seja $\left\{S_{k}\right\}$ uma seqüência de superfícies dadas por $S_{k}: t=\tau_{k}(x), k=1,2, \ldots$, satisfazendo $\tau_{k}(x)<$ $\tau_{k+1}(x)$ e $\lim _{k \rightarrow+\infty} \tau_{k}(x)=+\infty$. Assim, teremos o seguinte sistema diferencial impulsivo:

$$
\left\{\begin{array}{l}
\dot{x}=f(t, x), t \neq \tau_{k}(x) \\
\Delta x=I_{k}(x), \quad t=\tau_{k}(x), \quad k=1,2, \ldots
\end{array}\right.
$$

Note que os momentos de efeito impulsivo para o sistema (2.6) dependem das soluções, ou seja, eles variam de acordo com as soluções do sistema (2.6). Portanto, soluções iniciadas em diferentes pontos terão diferentes pontos de descontinuidades. Devido a isso, situações peculiares podem ocorrer, se comparados aos sistemas com momentos fixos de impulsos. Por exemplo, neste sistema, pode ocorrer de uma solução atingir a mesma superfície $t=\tau_{k}(x)$ várias vezes. Tal comportamento será denominado fenômeno de pulso. Além disso, soluções diferentes podem coincidir após algum tempo e se comportar como uma solução única após isso. Este fenômeno será chamado de confluência.

O exemplo seguinte ilustra estes comportamentos (que não ocorrem na teoria de EDOs clássicas).

Exemplo 2.17 ([21], Exemplo 1.1.3). Consideremos a equação diferencial impulsiva

$$
\left\{\begin{array}{l}
\dot{x}=0, t \neq \tau_{k}(x), t \geq 0 \\
\Delta x=x^{2} \operatorname{sgn}(x)-x, t=\tau_{k}(x), k=0,1,2, \ldots,
\end{array}\right.
$$

onde $\tau_{k}(x)=x+6 k$, com $|x|<3$, descreve a superfície $S_{k}: t=\tau_{k}(x)$, e

$$
\operatorname{sgn}(x)=\left\{\begin{array}{l}
1, x>0 \\
0, x=0 \\
-1, x<0
\end{array}\right.
$$

Note que as soluções $x(t)$ com condição inicial $x(0)=x_{0},\left|x_{0}\right| \geq 3$, não sofrem impulso, já que elas não encontram a superfície $S_{k}$, para qualquer $k=0,1,2, \ldots$

Por outro lado, as soluções $x(t)$ que se iniciam nos pontos $\left(0, x_{0}\right), 1<x_{0}<3$, sofrem efeito impulsivo um número finito de vezes. Por exemplo, considere a solução $x(t)$ com $x(0)=\sqrt[4]{2}$. Esta solução encontra a superfície $S_{0}$ três vezes e não se choca com qualquer superfície $S_{k}$ além do tempo $t_{3}=2$, como mostra a Figura 2.2. Se o ponto inicial $x(0)=x_{0}$ for tal que $0<x_{0}<1$, então a solução $x(t)$ encontrará a superfície $S_{k}$ em um número infinito de tempos $t_{k}$ e teremos $t_{k} \rightarrow+\infty$ quando $k \rightarrow+\infty$, bem como $\lim _{k \rightarrow+\infty} x\left(t_{k}\right)=0$. Para $x_{0}=\frac{1}{2}$, observe a Figura 2.3. 


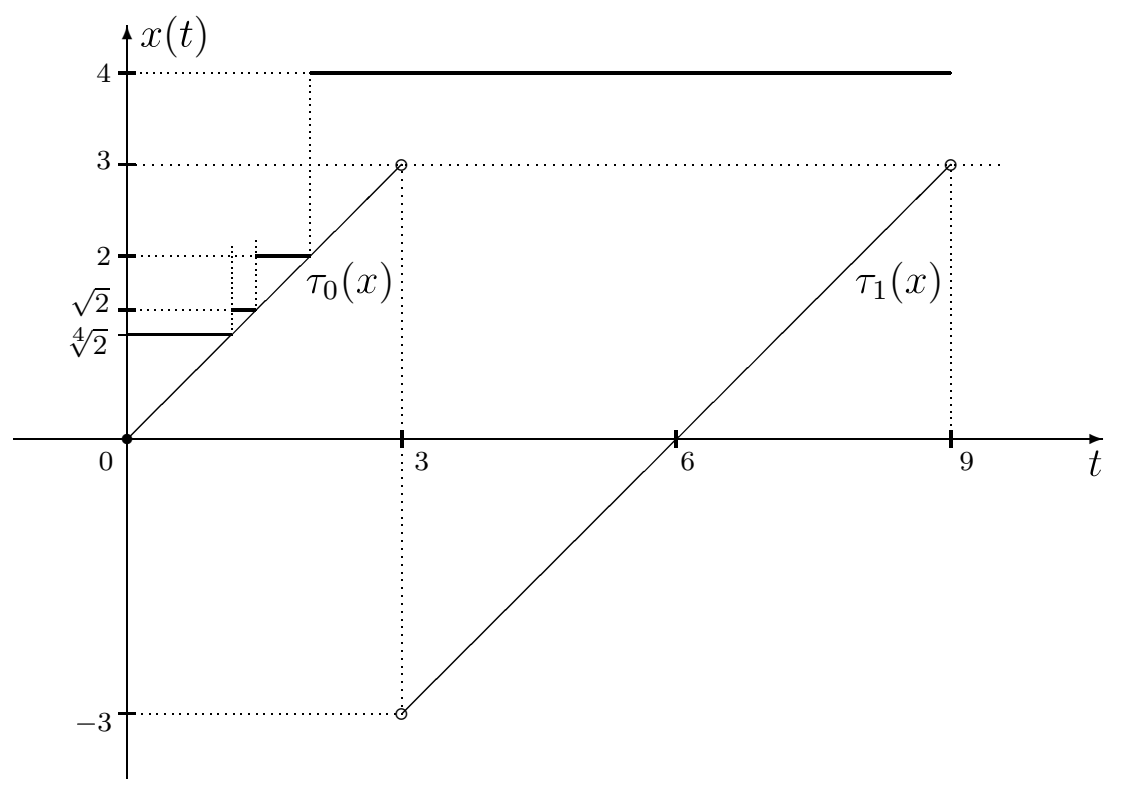

Figura 2.2: Curva integral do sistema impulsivo $(2.7) \operatorname{com} x(0)=\sqrt[4]{2}$.

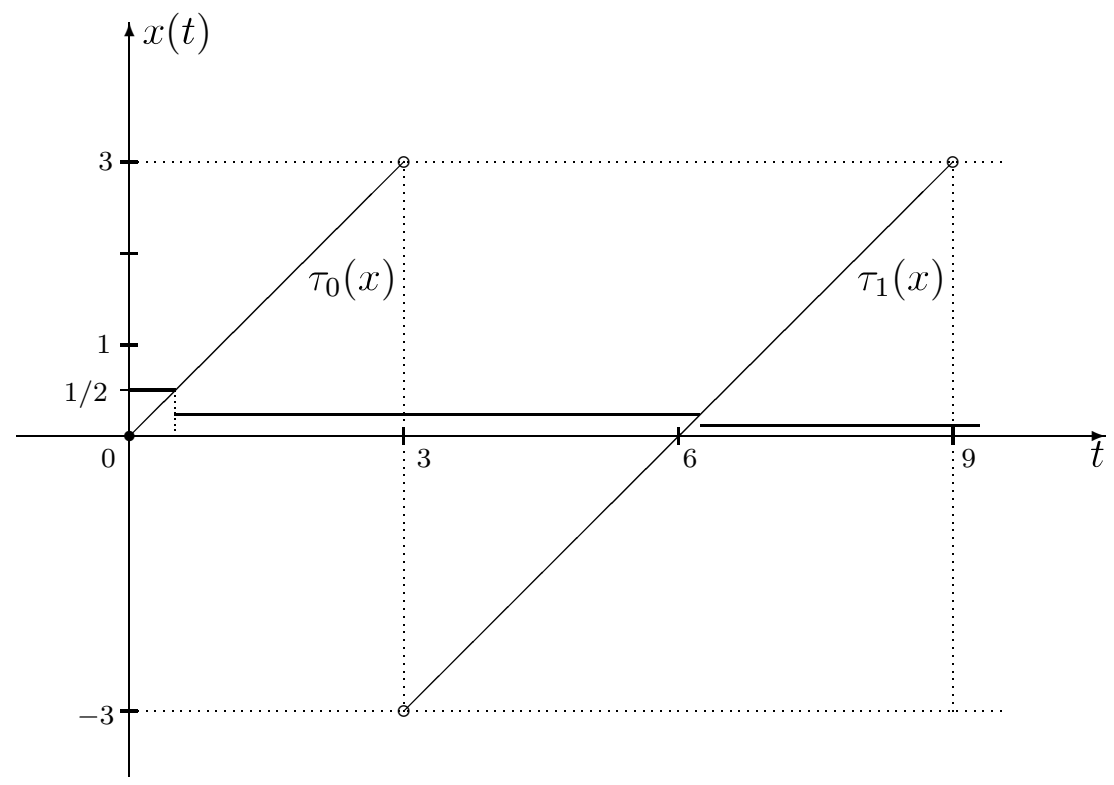

Figura 2.3: Curva integral para o sistema impulsivo $(2.7)$ com $x(0)=1 / 2$.

As soluções $x(t)$, com $-1<x_{0}<0$, se chocam um número infinito de tempos $t_{j}$, $j=1,2 \ldots$, com a mesma superfície $S_{k}$. Entretanto, neste caso, temos $\lim _{k \rightarrow+\infty} t_{k}=6 \mathrm{e}$ $\lim _{k \rightarrow+\infty} x\left(t_{k}\right)=0$, exibindo o fenômeno de pulso. Veja a Figura 2.4.

Finalmente, as soluções que começam em $(0, \sqrt[4]{2})$ e $(0,4)$ se unem em $t \geq 2$, exibindo o fenômeno de confluência.

Sistemas diferenciais impulsivos mais gerais do que (2.6) podem ser descritos da 


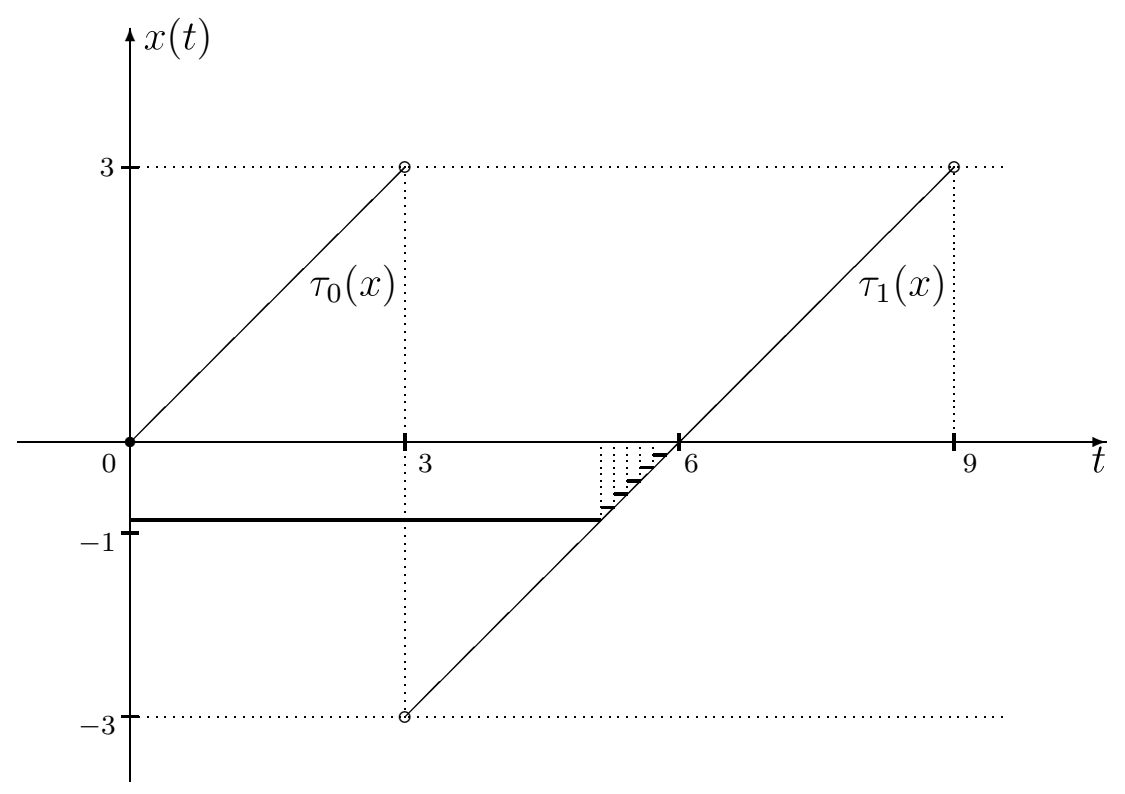

Figura 2.4: Curva integral do sistema impulsivo (2.7) para $-1<x(0)<0$.

maneira abaixo:

$$
\begin{cases}\dot{x}=f(t, x), & h(t, x) \neq 0 \\ \Delta x=I_{0}(t, x), & h(t, x)=0\end{cases}
$$

onde $h: \mathbb{R}_{+} \times \Omega \rightarrow \mathbb{R}^{n}$ é uma função e $\Omega$ é um subconjunto aberto de $\mathbb{R}^{n}$.

Se $h(t, x)=0$ tiver uma quantidade enumerável de raízes $t=\tau_{k}(x)$ para cada $x$, satisfazendo algumas condições necessárias, então, definindo $I_{k}(x)=I_{0}\left(\tau_{k}(x), x\right)$, poderemos notar que (2.8) se reduz a (2.6). Por outro lado, se $h(t, x)=0$ tiver raízes $\left\{\left(t_{k}, x\right): x \in M\left(t_{k}\right)\right\}$ tais que $\lim _{k \rightarrow+\infty} t_{k}=+\infty$, então (2.8) se reduzirá a (2.3).

A seguir, analisaremos sistemas autônomos com impulsos. Para isso, consideremos os conjuntos $M(t) \equiv M$ e $N(t) \equiv N$ independentes de $t$ e seja $A(t) \equiv A: M \rightarrow N$ definido por $A x=x+I(x)$, onde $I: \Omega \rightarrow \Omega$. Consideremos também o sistema diferencial impulsivo autônomo dado por

$$
\begin{cases}\dot{x}=f(x), & x \notin M, \\ \Delta x=I(x), & x \in M .\end{cases}
$$

Quando qualquer solução $x(t)=x\left(t ; t_{0}, x_{0}\right)$ de $(2.9)$ atingir o conjunto $M$ em algum tempo $t$, o operador $A$ transferirá, instantaneamente, o ponto $x(t) \in M$ para o ponto $y(t)=x(t)+I(x(t))$ que está em $N$. Como (2.9) é autônomo, o movimento do ponto $x(t)$ será considerado em $\Omega$ ao longo da trajetória do sistema (2.9). 
O exemplo seguinte mostra várias possibilidades interessantes.

Exemplo 2.18 ([21] - Exemplo 1.1.5). Consideremos o sistema diferencial impulsivo em $\mathbb{R}^{2}$ dado por

$$
\left\{\begin{array}{l}
\dot{x}_{1}=\alpha x_{1}-\beta x_{2}, \dot{x}_{2}=\beta x_{1}+\alpha x_{2}, \quad \alpha<0, \beta>0, \\
A: M \rightarrow N
\end{array}\right.
$$

onde os conjuntos $M, N \subset \mathbb{R}^{2}$ são definidos por

$$
M=\left\{\left(x_{1}, x_{2}\right) \in \mathbb{R}^{2}: x_{1}^{2}+x_{2}^{2}=\gamma_{1}^{2}\right\} \quad \text { e } \quad N=\left\{\left(x_{1}, x_{2}\right) \in \mathbb{R}^{2}: x_{1}^{2}+x_{2}^{2}=\gamma_{2}^{2}\right\}
$$

com $\gamma_{2}>\gamma_{1}$, e são tais que, para qualquer ponto $x \in M$, existe o correspondente $A x=$ $y \in N$ tal que $x$ e $y$ estão sobre a mesma semi-reta que se inicia na origem.

Usando coordenadas polares

$$
x_{1}=\rho \cos \theta \quad \text { e } \quad \mathrm{x}_{2}=\rho \operatorname{sen} \theta
$$

temos

$$
\frac{d x_{1}}{d t}=\frac{d \rho}{d t} \cos \theta-\rho \operatorname{sen} \theta \frac{d \theta}{d t} \quad \text { e } \quad \frac{d x_{2}}{d t}=\frac{d \rho}{d t} \operatorname{sen} \theta+\rho \cos \theta \frac{d \theta}{d t}
$$

e substituindo as coordenadas polares em (2.10), obtemos

$$
\frac{d x_{1}}{d t}=\alpha(\rho \cos \theta)-\beta(\rho \operatorname{sen} \theta) \quad \text { e } \quad \frac{d x_{2}}{d t}=\beta(\cos \theta)+\alpha(\rho \operatorname{sen} \theta)
$$

Assim, podemos reescrever o sistema (2.10) como

$$
\left\{\begin{array}{l}
\frac{d \rho}{d t}=\alpha \rho, \frac{d \theta}{d t}=\beta, \\
A:\left(\gamma_{1}, \theta\right) \rightarrow\left(\gamma_{2}, \theta\right),
\end{array}\right.
$$

onde

$$
M=\left\{(\rho, \theta): \rho=\gamma_{1}\right\} \quad \text { e } \quad N=\left\{(\rho, \theta): \rho=\gamma_{2}\right\}
$$

Note que as trajetórias do sistema diferencial correspondente são espirais logarítmas dadas por

$$
\rho=\rho_{0} \mathrm{e}^{(\alpha / \beta)\left(\theta-\theta_{0}\right)}, \quad \alpha<0, \beta>0,
$$

as quais tendem à posição estacionária $\rho=0$, quando $\theta \rightarrow+\infty$.

Note, também, que

$$
\frac{d \rho}{d t}=\alpha d t \Longrightarrow \ln \rho=\alpha t+C \Longrightarrow \rho=e^{\alpha t+C}
$$




$$
\frac{d \theta}{d t}=\beta \quad \Longrightarrow \quad d \theta=\beta d t \quad \Longrightarrow \quad \theta=\beta t+K
$$

Daí,

$$
\theta-K=\beta t \Longrightarrow \frac{\theta-K}{\beta}=t
$$

Logo

$$
\rho=e^{\alpha \frac{\theta-K}{\beta}+C}=e^{\frac{\alpha}{\beta}(\theta-K)} e^{C}
$$

Como

$$
\theta-K=\beta t \Longrightarrow \theta_{0}-K=\beta_{0} \Longrightarrow \theta_{0}=K,
$$

então vale

$$
\rho_{0}=e^{\frac{\alpha}{\beta}\left(\theta_{0}-\theta_{0}\right)} e^{c}=e^{c}
$$

e, portanto,

$$
\rho=e^{\frac{\alpha}{\beta}\left(\theta-\theta_{0}\right)} \rho_{0} .
$$

Como todas as trajetórias do sistema diferencial impulsivo (2.10) que se iniciam na região $\rho>\gamma_{1}$ encontram o círculo $\rho=\gamma_{2}$, é suficiente considerarmos as trajetórias iniciando sobre o círculo $N$. A trajetória $\rho=\gamma_{2} \mathrm{e}^{(\alpha / \beta)\left(\theta-\theta_{0}\right)}$, iniciando em $\left(\gamma_{2}, \theta_{0}\right)$, encontra o círculo $M$ em $\left(\gamma_{1}, \theta_{1}\right)$, onde

$$
\theta_{1}=\theta_{0}+\beta t_{1} \equiv \theta_{0}+\tau
$$

$\operatorname{com} \tau=\frac{\beta}{\alpha} \ln \frac{\gamma_{1}}{\gamma_{2}}$. De fato, pois

$$
\rho(\theta)=\gamma_{1} \Longrightarrow \gamma_{1}=\gamma_{2} \mathrm{e}^{(\alpha / \beta)\left(\theta-\theta_{0}\right)} \Longrightarrow \theta=\theta_{1}=\theta_{0}+\frac{\beta}{\alpha} \ln \frac{\gamma_{1}}{\gamma_{2}}=\theta_{0}+\tau
$$

Note, ainda, que o operador $A$ leva $\left(\gamma_{1}, \theta_{1}\right)$ sobre $N$. Desta forma, o movimento ocorre ao longo da espiral $\gamma_{2} \mathrm{e}^{(\alpha / \beta)\left(\theta-\theta_{1}\right)}$ até encontrar $M$, digamos em $\left(\gamma_{1}, \theta_{2}\right)$, o qual é transferido pelo operador $A$ para o ponto $\left(\gamma_{2}, \theta_{2}\right)$ sobre $N$, com $\theta_{2}=\theta_{0}+2 \tau$. Assim, depois do $n$ ésimo encontro com $M$, o movimento estará sobre o ponto $\left(\gamma_{2}, \theta_{0}+n \tau\right)$. Por isso, para se estudar o movimento das trajetórias, é suficiente considerarmos a distribuição de todos os pontos $\theta_{n}=\theta_{0}+n \tau$ sobre o círculo $N$.

Se $\frac{\tau}{2 \pi}$ for racional, isto é, se $\tau=2 \pi \frac{p}{q}$, com $p$ e $q$ inteiros positivos relativamente primos, então teremos

$$
\theta_{q}=\theta_{0}+q \tau \equiv \theta_{0} \quad(\bmod 2 \pi)
$$

e o movimento da trajetória será periódico. Além disso, como $\theta_{0}$ é arbitrário, então todas 
as trajetórias iniciando em $N$ serão periódicas.

Se $\frac{\tau}{2 \pi}$ for irracional, dividimos o círculo $N$ em $k$ arcos iguais, cada um de comprimento $\frac{2 \pi}{k}$. Pelo fato de $\frac{\tau}{2 \pi}$ não ser racional, nunca teremos $\theta_{n} \equiv \theta_{0}(\bmod 2 \pi)$. Daí, entre os $(k+1)$ primeiros pontos, $\theta_{0}, \theta_{0}+\tau, \ldots, \theta_{0}+k \tau(\bmod 2 \pi)$, existirão dois pontos sobre o mesmo arco, digamos $\theta_{0}+m \tau$ e $\theta_{0}+l \tau, \operatorname{com} m>l$.

Seja $s=m-l$. Como $\theta_{0}+m \tau$ e $\theta_{0}+l \tau$ estão sobre o mesmo arco, então

$$
\left(\theta_{0}+m \tau\right)-\left(\theta_{0}+l \tau\right)<\frac{2 \pi}{k}
$$

Desta forma, o ângulo $s \tau$ é menor do que $\frac{2 \pi}{k}$. Assim, na sequência $\left\{\theta_{0}+n s \tau(\bmod 2 \pi)\right\}$, $n \in \mathbb{N}$, qualquer dois ângulos consecutivos diferem pelo mesmo número $s \tau<\frac{2 \pi}{k}$.

Então dados $\varepsilon>0$ arbitrário e $k$ suficientemente grande, podemos obter $\frac{2 \pi}{k}<\varepsilon$. Logo, dada qualquer $\varepsilon$-vizinhança de qualquer ponto sobre o círculo $N$, existe pelo menos um elemento do conjunto $\left\{\theta_{0}+n s \tau: n \in \mathbb{N}\right\}$.

Assim mostramos que as trajetórias iniciando na região $x_{1}^{2}+x_{2}^{2}>\gamma_{1}^{2}$ são densas no anel $\gamma_{1}^{2}<x_{1}^{2}+x_{2}^{2}<\gamma_{2}^{2}$.

\subsection{Noções básicas de EDFRIs}

Listaremos, a seguir, algumas notações essenciais para iniciarmos o nosso estudo sobre equações diferenciais funcionais retardadas impulsivas (EDFRIs).

Dada uma função $\psi:[a, b] \rightarrow \mathbb{R}^{n},[a, b] \subset \mathbb{R}$, usaremos a seguinte notação

$$
\psi\left(t^{+}\right)=\lim _{s \rightarrow t^{+}} \psi(s) \quad \text { e } \quad \psi\left(t^{-}\right)=\lim _{s \rightarrow t^{-}} \psi(s)
$$

para indicarmos, respectivamente, os limites laterais à direita e à esquerda de $\psi$ em $t$, quando existirem.

Sejam $a, b \in \mathbb{R}$ com $a<b$ e $D \subseteq \mathbb{R}^{n}$. Denotaremos por $P C([a, b], D)$ o espaço formado pelas funções $\psi:[a, b] \rightarrow D$ que são contínuas exceto em um número finito de pontos e cujos limites laterais $\psi\left(t^{+}\right)$e $\psi\left(t^{-}\right)$existem, satisfazendo $\psi\left(t^{-}\right)=\psi(t)$, para todo $t \in[a, b)$.

Denotaremos por $P C([a, \infty), D)$ o espaço das funções $\psi:[a, \infty) \rightarrow D$ tais que para todo $c>a$, a restrição $\left.\psi\right|_{[a, c]} \in P C([a, c], D)$. Em $P C([a, b], D)$, consideraremos a norma usual do supremo, denotando-a por $\|\cdot\|$ e em $P C([a, \infty), D)$, consideraremos a topologia da convergência localmente uniforme.

Se $x \in P C\left(\left[t_{0}-r, \sigma\right], \mathbb{R}^{n}\right)$, onde $t_{0} \in \mathbb{R}_{+}, r>0$ e $\sigma \geq t_{0}$, então, para cada $t \in\left[t_{0}, \sigma\right]$, 
definiremos $x_{t} \in P C\left([-r, 0], \mathbb{R}^{n}\right)$ da seguinte forma

$$
x_{t}(s)=x(t+s), \quad-r \leq s \leq 0 .
$$

Sejam $J \subset \mathbb{R}^{+}$um intervalo da forma $[a, b)$, com $0 \leq a<b \leq \infty$, e $D \subset \mathbb{R}^{n}$ um conjunto aberto.

Consideremos a sequência

$$
0 \leq t_{0}<t_{1}<t_{2}<\ldots<t_{k}<\ldots
$$

de pontos satisfazendo $\lim _{k \rightarrow \infty} t_{k}=\infty$ e o seguinte problema de valor inicial (PVI)

$$
\left\{\begin{array}{l}
\dot{x}(t)=f\left(t, x_{t}\right), \quad t \geq t_{0}, \quad t \neq t_{k} \\
x_{t_{0}}=\phi
\end{array}\right.
$$

onde $t_{0} \in \mathbb{R}_{+}$e $\phi \in P C\left([-r, 0], \mathbb{R}^{n}\right)$.

Consideremos, também, nos instantes de impulsos $t_{k}, k=1,2, \ldots$, a seguinte condição de impulso

$$
\Delta x(t)=I\left(t, x_{t}\right), \quad t>t_{0}, \quad t=t_{k},
$$

onde $\Delta x\left(t_{k}\right)=x\left(t_{k}{ }^{+}\right)-x\left(t_{k}\right), f, I: J \times P C([-r, 0], D) \rightarrow \mathbb{R}^{n}$ e $\psi(0)+I\left(t_{k}, \psi\right) \in D$, para todo $\left(t_{k}, \psi\right) \in J \times P C([-r, 0], D)$.

Definição 2.19. Seja $\left[t_{0}, t_{0}+\alpha\right] \subset J$, com $\alpha>0$. Uma solução do problema impulsivo (2.11)-(2.12) em $\left[t_{0}, t_{0}+\alpha\right]$ é uma função $x \in P C\left(\left[t_{0}-r, t_{0}+\alpha\right], D\right)$ que satisfaz as seguintes propriedades:

(a) $x(t)$ é contínua em $\left[t_{0}, t_{0}+\alpha\right] \backslash\left\{t_{k} ; k \in \mathbb{N}\right\}$, os limites laterais $x\left(t_{k}^{-}\right)$e $x\left(t_{k}^{+}\right)$existem e $x(t)$ é contínua à esquerda em $t_{k} \in\left[t_{0}, t_{0}+\alpha\right], k=1,2, \ldots$;

(b) $x(t)$ satisfaz a equação (2.11), para todo $t \in\left[t_{0}, t_{0}+\alpha\right]$;

(c) $x\left(t_{k}\right)$, com $t_{k} \leq t_{0}+\alpha$, satisfaz a equação (2.12), para todo $k \in \mathbb{N}$.

Denotaremos uma solução de (2.11)-(2.12) por $x(t)=x\left(t ; t_{0}, \phi\right)$ ou simplesmente por $x=x\left(t_{0}, \phi\right)$.

Note que a solução $x(t)$ coincide com $\phi\left(t-t_{0}\right)$, para $t_{0}-r \leq t \leq t_{0}$. Sendo assim, se uma solução $x(t)$ de (2.11)-(2.12) existir em $\left[t_{0}-r, t_{0}+\alpha\right]$ e sofrer efeitos impulsivos nos 
instantes $\left\{t_{k}\right\}_{k=1}^{m}$, onde $t_{0}<t_{1}<t_{2}<\ldots<t_{m} \leq t_{0}+\alpha$, então ela será descrita por

$$
x(t)= \begin{cases}x\left(t ; t_{0}, \phi\right), & t \in\left[t_{0}-r, t_{1}\right], \\ x\left(t ; t_{k}, x_{t_{k}}\right), & t \in\left(t_{k}, t_{k+1}\right], \quad k=1,2, \ldots, m-1, \\ x\left(t ; t_{m}, x_{t_{m}}\right), & t \in\left(t_{m}, t_{0}+\alpha\right] .\end{cases}
$$

Entretanto, se uma solução $x(t)$ existir sobre o intervalo $\left[t_{0}-r, \infty\right)$, então $x(t)$ sofrerá infinitos impulsos nos instantes $\left\{t_{k}\right\}_{k=1}^{\infty}$, onde $t_{0}<t_{1}<t_{2}<\ldots<t_{k}<\ldots$ satisfazendo $\lim _{k \rightarrow \infty} t_{k}=\infty$, isto é, os instantes de impulsos não se acumulam. Neste caso, a solução será descrita da seguinte maneira

$$
x(t)= \begin{cases}x\left(t ; t_{0}, \phi\right), & t \in\left[t_{0}-r, t_{1}\right], \\ x\left(t ; t_{k}, x_{t_{k}}\right), & t \in\left(t_{k}, t_{k+1}\right], \quad k=1,2, \ldots,\end{cases}
$$

ou seja, $x\left(t ; t_{k}, x_{t_{k}}\right)$ representa uma solução de $(2.11)$-(2.12), para cada $k \in \mathbb{N}$ e cada $t \in\left(t_{k}, t_{k+1}\right]$, onde $t_{k}$ denota o instante inicial e $x_{t_{k}}$ representa a função inicial.

Para os próximos resultados, consideraremos $t_{0} \in J$ e $\alpha>0$ tais que $\left[t_{0}, t_{0}+\alpha\right] \subset J$. Iremos supor, também, que a função $f: J \times P C([-r, 0], D) \rightarrow \mathbb{R}^{n}$ satisfaça as seguintes condições:

(i) Para cada $x \in P C\left(\left[t_{0}-r, t_{0}+\alpha\right), D\right)$, a função $t \mapsto f\left(t, x_{t}\right)$ pertence a $P C\left(\left[t_{0}, t_{0}+\right.\right.$ $\left.\alpha), \mathbb{R}^{n}\right)$.

(ii) Para cada $t \in J, f(t, \psi)$ é uma função contínua em $\psi$, onde $\psi \in P C([-r, 0], D)$.

(iii) Para cada conjunto compacto $F \subset D$, existe $M>0$ tal que $|f(t, \psi)| \leq M$, para todo $\operatorname{par}(t, \psi) \in\left[t_{0}, t_{0}+\alpha\right] \times P C([-r, 0], F)$.

O próximo lema exibirá uma fórmula integral para uma solução de (2.11)-(2.12).

Lema 2.20 ([10], Lema 1.9). Consideremos o problema (2.11)-(2.12), onde $f$ satisfaz a condição $(i)$ acima. Então $x \in P C\left(\left[t_{0}-r, t_{0}+\alpha\right], D\right)$, onde $\alpha>0 e\left[t_{0}-r, t_{0}+\alpha\right] \subset J$, 
será uma solução de (2.11)-(2.12) se, e somente se,

$$
x(t)= \begin{cases}\phi\left(t-t_{0}\right), & t \in\left[t_{0}-r, t_{0}\right], \\ \phi(0)+\int_{t_{0}}^{t} f\left(s, x_{s}\right) d s, & t \in\left[t_{0}, t_{1}\right], \\ x\left(t_{k}\right)+I\left(t_{k}, x_{t_{k}}\right)+\int_{t_{k}}^{t} f\left(s, x_{s}\right) d s, & t \in\left(t_{k}, t_{k+1}\right], \quad k=1,2, \cdots, m-1, \\ x\left(t_{m}\right)+I\left(t_{m}, x_{t_{m}}\right)+\int_{t_{m}}^{t} f\left(s, x_{s}\right) d s, & t \in\left(t_{m}, t_{0}+\alpha\right]\end{cases}
$$

ou equivalentemente,

$$
x(t)=\left\{\begin{array}{l}
\phi\left(t-t_{0}\right), \quad t \in\left[t_{0}-r, t_{0}\right], \\
\phi(0)+\int_{t_{0}}^{t} f\left(s, x_{s}\right) d s+\sum_{t_{0}<t_{k}<t} I\left(t_{k}, x_{t_{k}}\right), \quad t \in\left(t_{0}, t_{0}+\alpha\right] .
\end{array}\right.
$$

O próximo teorema garante a existência de uma solução local de (2.11)-(2.12).

Teorema 2.21 (Existência local - [10], Teorema 1.12). Consideremos o problema (2.11)(2.12) e suponhamos que $f$ satisfaça as condições $(i)$ a (iii). Então, para cada $\left(t_{0}, \phi\right) \in$ $J \times P C\left([-r, 0], \mathbb{R}^{n}\right)$, existe uma solução $x=x\left(t ; t_{0}, \phi\right)$ de $(2.11)-(2.12)$ em $\left[t_{0}-r, t_{0}+\alpha\right]$, para algum $\alpha>0$.

Não incluiremos a demonstração deste resultado, devido à sua extensão, mas uma prova pode ser encontrada em [10], Teorema 1.12.

Definição 2.22. Se $x$ e y forem soluções de (2.11)-(2.12) sobre os intervalos $J_{1}$ e $J_{2}$, respectivamente, onde $J_{1}$ está propriamente contido em $J_{2}$ e estes intervalos são semiabertos à direita, e se $x(t)=y(t)$ para todo $t \in J_{1}$, então y será uma continuação própria à direita de $x$, ou simplesmente, uma continuação de $x$. Uma solução $x$ de (2.11)-(2.12) definida sobre $J_{1}$ será dita continuável, se existir alguma continuação y de $x$. Caso contrário, $x$ será não-continuável e o intervalo $J_{1}$ será chamado de intervalo maximal de existência de $x$.

É consequência imediata do Lema de Zorn que se $x$ for uma solução continuável de (2.11)-(2.12) sobre algum intervalo, então $x$ poderá ser continuada a um intervalo maximal de existência. 
Mostraremos, a seguir, alguns resultados que abordam a continuação de soluções de (2.11)-(2.12) ao intervalo maximal de existência.

Teorema 2.23 (Continuação - [10], Teorema 1.14). Consideremos o problema impulsivo (2.11)-(2.12) e suponhamos que $f$ satisfaça as condições $(i)$ a $(i i i)$. Sejam $\left(t_{0}, \phi\right) \in J \times$ $P C([-r, 0], D)$ e $x=x\left(t_{0}, \phi\right)$ uma solução de (2.11)-(2.12). Se $x$ estiver definida em $\left[t_{0}-r, t_{0}+\alpha\right]$, onde $\alpha>0 e\left[t_{0}, t_{0}+\alpha\right] \subset J$, então $x$ será continuável. Agora, se $x$ estiver definida sobre um intervalo da forma $\left[t_{0}-r, t_{0}+\beta\right]$, onde $0<\beta<\infty e\left[t_{0}, t_{0}+\beta\right] \subset J$, e $x$ for não continuável, então para todo conjunto compacto $G \subset D$, existirá uma sequência de números $\left\{t_{k}\right\}_{k=1}^{\infty}$ com $t_{0}<t_{k}<t_{k+1} e \lim _{k \rightarrow \infty} t_{k}=t_{0}+\beta$ tal que $x\left(t_{k}\right) \notin G$.

Pela primeira parte do teorema acima, segue que o intervalo maximal de existência de uma solução não continuável de (2.11)-(2.12) é aberto à direita. Já, pela segunda parte, uma solução não continuável $x$ ou está definida para todo $t \in J$ ou está definida sobre um subintervalo próprio limitado de $J$ e, neste caso, a solução ou se torna ilimitada quando $t \rightarrow t_{0}+\beta$, isto é, lim $\sup _{t \rightarrow t_{0}+\beta}|x(t)|=\infty$, ou ela assume valores suficientemente próximos da fronteira de $D$ quando $t \rightarrow t_{0}+\beta$. No caso particular em que $J=\mathbb{R}^{+}$e $D=\mathbb{R}^{n}$, as soluções limitadas de (2.11)-(2.12) são continuáveis a $t=\infty$.

Teorema 2.24 (Existência global - [10], Teorema 1.17). Suponhamos que f satisfaça as condições (i) a (iii) e também satisfaça a seguinte condição:

(iv) Existem funções $h_{1}, h_{2} \in P C\left(\mathbb{R}_{+}, \mathbb{R}_{+}\right)$tais que, para $(t, \psi) \in \mathbb{R}_{+} \times P C\left([-r, 0], \mathbb{R}^{n}\right)$, temos

$$
|f(t, \psi)| \leq h_{1}(t)+h_{2}(t)\|\psi\|
$$

Então, para cada $\left(t_{0}, \phi\right) \in \mathbb{R}_{+} \times P C\left([-r, 0], \mathbb{R}^{n}\right)$, existirá uma solução (local) $x=x\left(t ; t_{0}, \phi\right)$ de (2.11)-(2.12) que pode ser estendida ao intervalo $\left[t_{0}-r, \infty\right)$.

Definição 2.25. Uma solução $x=x\left(t ; t_{0}, \phi\right)$ de (2.11)-(2.12) será dita única, se dada qualquer outra solução $y=y\left(t ; t_{0}, \phi\right)$ de $(2.11)-(2.12)$, tivermos $x(t)=y(t)$ sobre o intervalo comum de existência.

O teorema a seguir trata da unicidade de soluções de (2.11)-(2.12).

Teorema 2.26 (Unicidade - [10], Teorema 1.19). Suponhamos que f satisfaça a condição (i) e a condição abaixo

(v) $f: J \times P C([-r, 0], D) \rightarrow \mathbb{R}^{n}$ é localmente lipschtiziana em $\psi$, isto é, para cada $t_{0} \in J$ e cada $\alpha>0$, onde $\left[t_{0}, t_{0}+\alpha\right] \subset J$, e para cada compacto $F \subset D$, existe uma 
constante $L>0$ tal que

$$
\left|f\left(t, \psi_{1}\right)-f\left(t, \psi_{2}\right)\right| \leq L\left\|\psi_{1}-\psi_{2}\right\|
$$

para quaisquer $t \in\left[t_{0}, t_{0}+\alpha\right]$ e $\psi_{1}, \psi_{2} \in P C([-r, 0], F)$.

Se existir uma solução de (2.11)-(2.12) em $\left[t_{0}-r, t_{0}+\alpha\right)$, onde $0<\alpha \leq \infty e\left[t_{0}, t_{0}+\alpha\right) \subset J$, então ela será única.

\subsection{EDFRIs num contexto mais geral}

Nesta seção, vamos considerar o seguinte problema de valor inicial para uma EDFRI

$$
\left\{\begin{array}{l}
\dot{y}(t)=f\left(y_{t}, t\right), t \neq t_{k} \\
\Delta y\left(t_{k}\right)=I_{k}\left(y\left(t_{k}\right)\right), k=0,1, \ldots, m \\
y_{t_{0}}=\phi
\end{array}\right.
$$

onde $t_{k}, k=0,1, \ldots, m$, com $t_{0}<t_{1}<\ldots<t_{k}<\ldots<t_{m} \leq t_{0}+\sigma, \sigma>0$, são momentos de impulsos pré determinados, para $k=0,1, \ldots, m, y \mapsto I_{k}(y)$ aplica $\mathbb{R}^{n}$ nele mesmo e

$$
\Delta y\left(t_{k}\right):=y\left(t_{k}+\right)-y\left(t_{k}-\right)=y\left(t_{k}+\right)-y\left(t_{k}\right),
$$

ou seja, $y$ é contínua à esquerda em $t=t_{k}$ e limite lateral $y\left(t_{k}+\right)$ existe, para $k=$ $0,1,2, \ldots, m$. Note que também estamos considerando $t_{0}$ como momento de impulso.

Dada uma função $y:\left[t_{0}-r, t_{0}+\sigma\right] \rightarrow \mathbb{R}^{n}$, com $r>0$ e $\sigma>0$, consideraremos $y_{t}:[-r, 0] \rightarrow \mathbb{R}^{n}$ dada por

$$
y_{t}(\theta)=y(t+\theta), \quad \theta \in[-r, 0], t \in\left[t_{0}, t_{0}+\sigma\right] .
$$

Agora, iremos especificar o conjunto que contém a função $f$ do problema (2.13).

Seja $X$ um espaço de Banach. Escreveremos $f \in G([a, b], X)$ se $f$ for uma função regrada que aplica $[a, b]$ em $X$ e consideraremos, neste conjunto, a norma usual do supremo dada por $\|f\|_{\infty}=\sup _{a \leq t \leq b}\|f(t)\|$. Então $\left(G([a, b], X),\|\cdot\|_{\infty}\right)$ será um espaço de Banach. Como mencionamos no Capítulo 1 , toda função em $G([a, b], X)$ é o limite uniforme de funções escada finitas (veja [16]).

Definamos o conjunto

$$
G^{-}([a, b], X)=\{u \in G([a, b], X): u \text { é contínua à esquerda para todo } t \in(a, b]\}
$$


Em $G^{-}([a, b], X)$, consideraremos a norma induzida por $G([a, b], X)$. Desta forma, está claro que para uma dada função $y \in G^{-}\left(\left[t_{0}-r, t_{0}+\sigma\right], \mathbb{R}^{n}\right), y_{t} \in G^{-}\left([-r, 0], \mathbb{R}^{n}\right)$ para todo $t \in\left[t_{0}, t_{0}+\sigma\right]$.

É um fato conhecido que o sistema impulsivo (2.13) é equivalente à equação integral

$$
\left\{\begin{array}{l}
y(t)=y\left(t_{0}\right)+\int_{t_{0}}^{t} f\left(y_{s}, s\right) d s+\sum_{t_{0} \leq t_{k}<t} I_{k}\left(y\left(t_{k}\right)\right) \\
y_{t_{0}}=\phi
\end{array}\right.
$$

sempre que a integral existir em algum sentido. Em nosso caso, consideraremos a integral no sentido de Lebesgue.

Seja $P C_{1} \subset G^{-}\left(\left[t_{0}-r, t_{0}+\sigma\right], \mathbb{R}^{n}\right)$ um conjunto aberto (com a topologia da convergência localmente uniforme) com a seguinte propriedade: se $y$ for um elemento de $P C_{1}$ e $\bar{t} \in\left[t_{0}-r, t_{0}+\sigma\right]$, então $\bar{y}$ será dada por

$$
\bar{y}(t)=\left\{\begin{array}{l}
y(t), t_{0}-r \leq t \leq \bar{t} \\
y(\bar{t}), \bar{t}<t \leq \infty
\end{array}\right.
$$

e $\bar{y}$ também será um elemento de $P C_{1}$. Em particular, toda bola aberta em $G^{-}\left(\left[t_{0}-\right.\right.$ $\left.\left.r, t_{0}+\sigma\right], \mathbb{R}^{n}\right)$ tem esta propriedade.

Suponhamos que $f(\psi, t): G^{-}\left([-r, 0], \mathbb{R}^{n}\right) \times\left[t_{0}, t_{0}+\sigma\right] \rightarrow \mathbb{R}^{n}$ seja uma função dada. Suponhamos, também, que a aplicação $t \mapsto f\left(y_{t}, t\right)$ seja Lebesgue integrável para $y \in P C_{1}$ e que as seguintes condições aconteçam:

(A) existe uma função Lebesgue integrável $M:\left[t_{0}, t_{0}+\sigma\right] \rightarrow \mathbb{R}$ tal que para todo $x \in P C_{1}$ e todo $u_{1}, u_{2} \in\left[t_{0}, t_{0}+\sigma\right]$,

$$
\left|\int_{u_{1}}^{u_{2}} f\left(x_{s}, s\right) d s\right| \leq \int_{u_{1}}^{u_{2}} M(s) d s
$$

$(B)$ existe uma função Lebesgue integrável $L:\left[t_{0}, t_{0}+\sigma\right] \rightarrow \mathbb{R}$ tal que para todo $x, y \in$ $P C_{1}$ e todo $u_{1}, u_{2} \in\left[t_{0}, t_{0}+\sigma\right]$,

$$
\left|\int_{u_{1}}^{u_{2}}\left[f\left(x_{s}, s\right)-f\left(y_{s}, s\right)\right] d s\right| \leq \int_{u_{1}}^{u_{2}} L(s)\left\|x_{s}-y_{s}\right\| d s
$$

Para os operadores de impulsos $I_{k}: \mathbb{R}^{n} \rightarrow \mathbb{R}^{n}, k=0,1,2, \ldots$, suponhamos que as seguintes condições estejam satisfeitas: 
$\left(A^{\prime}\right)$ existe uma constante $K_{1}>0$ tal que para todo $k=0,1,2, \ldots$, e todo $x \in \mathbb{R}^{n}$,

$$
\left|I_{k}(x)\right| \leq K_{1}
$$

$\left(B^{\prime}\right)$ existe uma constante $K_{2}>0$ tal que para todo $k=0,1,2, \ldots$, e quaisquer $x, y \in \mathbb{R}^{n}$,

$$
\left|I_{k}(x)-I_{k}(y)\right| \leq K_{2}|x-y|
$$

Note que as condições do tipo Carathéodory e Lipschitz $(A)$ e $(B)$ são requeridas para a integral indefinida de $f$ e não para a função $f$. Portanto, a hipótese usual de que $f(\psi, t)$ deve ser contínua em $\psi$ não necessita estar satisfeita. Além disso, a aplicação $t \mapsto f\left(y_{t}, t\right)$ não precisa ser contínua por partes; basta ser Lebesgue integrável.

Note que, opcionalmente, podemos considerar outra integral em lugar da integral de Lebesgue, como por exemplo, a integral não absoluta de Henstock-Kurzweil. Se este for o caso, então a aplicação $t \mapsto f\left(y_{t}, t\right)$ poderá não somente ter muitos pontos de descontinuidades, mas ela também poderá ser de variação ilimitada. Em qualquer caso, usandose integração de Lebesgue ou integração de Henstock-Kurzweil, a solução da equação (2.13) precisa estar satisfeita quase sempre no sentido da medida de Lebesgue.

Vamos, então, relembrar o conceito de uma solução do problema (2.13).

Definição 2.27. Um função $y \in P C_{1}$ tal que $\left(y_{t}, t\right) \in G^{-}\left([-r, 0], \mathbb{R}^{n}\right) \times\left[t_{0}, t_{0}+\sigma\right]$ para $t_{0} \leq t<t_{0}+\sigma$ e tal que as condições

(i) $\dot{y}(t)=f\left(y_{t}, t\right)$, quase sempre (no sentido de Lebesgue), para $t \neq t_{k}$.

(ii) $y\left(t_{k}+\right)=y\left(t_{k}\right)+I_{k}\left(y\left(t_{k}\right)\right), k=0,1,2, \ldots, m$.

(iii) $y_{t_{0}}=\phi$

estão satisfeitas, será dita uma solução de (2.13) em $\left[t_{0}, t_{0}+\sigma\right]$ (ou algumas vezes também $\left.e m\left[t_{0}-r, t_{0}+\sigma\right]\right)$ com condição inicial $\left(\phi, t_{0}\right)$.

No que segue, apresentaremos um teorema de existência local e unicidade de soluções para a EDFRI (2.13). Daremos uma prova direta sem fazermos uso da teoria de EDOGs. Nossa demonstração, entretanto, foi inspirada na demonstração do Teorema 2.15 de [7] (veja, também, o Teorema 1.28 do Capítulo 1).

Teorema 2.28. Consideremos o problema (2.13) e suponhamos que as condições $(A)$, $(B),\left(A^{\prime}\right)$ e $\left(B^{\prime}\right)$ estejam satisfeitas. Então existirá um $\Delta>0$ tal que no intervalo $\left[t_{0}, t_{0}+\right.$ $\Delta]$ existirá uma única solução y $\left[t_{0}-r, t_{0}+\Delta\right] \rightarrow \mathbb{R}^{n}$ do problema (2.13) com $y_{t_{0}}=\phi$. 
Prova: Para $t \in\left[t_{0}, t_{0}+\sigma\right]$, definamos

$$
h_{1}(t)=\int_{t_{0}}^{t}[M(s)+L(s)] d s \quad \text { e } \quad h_{2}(t)=\max \left(K_{1}, K_{2}\right) \sum_{k=0}^{m} H_{t_{k}}(t),
$$

onde $H_{t_{k}}$ denota a função Heaviside contínua à esquerda e concentrada em $t_{k}$, ou seja,

$$
H_{t_{k}}(t)=\left\{\begin{array}{l}
0, \text { para } t \leq t_{k} \\
1, \text { para } t_{k}<t
\end{array}\right.
$$

Seja $h=h_{1}+h_{2}$. Então $h$ será não decrescente e contínua à esquerda. Além disso, para $s \in\left[t_{0}, t_{0}+\sigma\right]$, temos

$$
\begin{gathered}
\left|\int_{t_{0}}^{s} f\left(y_{t}, t\right) d t+\sum_{t_{0} \leq t_{j}<s} I_{j}\left(y\left(t_{j}\right)\right)\right|=\left|\int_{t_{0}}^{s} f\left(y_{t}, t\right) d t+\sum_{j=0}^{m} I_{j}\left(y\left(t_{j}\right)\right) H_{t_{j}}(s)\right| \leq \\
\leq \int_{t_{0}}^{s} M(t) d t+K_{1} \sum_{j=0}^{m} H_{t_{j}}(s) \leq h(s)-h\left(t_{0}\right),
\end{gathered}
$$

pelas condições $(A)$ e $\left(A^{\prime}\right)$.

Consideraremos dois casos: quando $t_{0}$ é um ponto de continuidade de $h:\left[t_{0}, t_{0}+\sigma\right] \rightarrow$ $\mathbb{R}^{n}$ e caso contrário.

Primeiramente, seja $t_{0}$ um ponto de continuidade de $h$. Suponhamos que $\Delta>0$ seja tal que $\left[t_{0}, t_{0}+\Delta\right] \subset\left[t_{0}, t_{0}+\sigma\right)$ e $h\left(t_{0}+\Delta\right)-h\left(t_{0}\right)<\frac{1}{2}$.

Seja $Q$ o conjunto de funções $z:\left[t_{0}, t_{0}+\Delta\right] \rightarrow \mathbb{R}^{n}$ tal que $z \in B V\left(\left[t_{0}, t_{0}+\Delta\right], \mathbb{R}^{n}\right)$ e $\left|z(t)-y\left(t_{0}\right)\right| \leq h(t)-h\left(t_{0}\right)$ para $t \in\left[t_{0}, t_{0}+\Delta\right]$. É fácil mostrar que o conjunto $Q \subset B V\left(\left[t_{0}, t_{0}+\Delta\right], \mathbb{R}^{n}\right)$ é fechado.

Para $s \in\left[t_{0}, t_{0}+\Delta\right]$ e $z \in Q$, definamos

$$
T z(s)=y\left(t_{0}\right)+\int_{t_{0}}^{s} f\left(z_{t}, t\right) d t+\sum_{t_{0} \leq t_{j}<s} I_{j}\left(z\left(t_{j}\right)\right) .
$$

Então,

$$
\left|T z(s)-y\left(t_{0}\right)\right|=\left|\int_{t_{0}}^{s} f\left(z_{t}, t\right) d t+\sum_{t_{0} \leq t_{j}<s} I_{j}\left(z\left(t_{j}\right)\right)\right| \leq h(s)-h\left(t_{0}\right), \quad s \in\left[t_{0}, t_{0}+\Delta\right] .
$$

O fato de que $T z$ pertence a $B V\left(\left[t_{0}, t_{0}+\Delta\right], \mathbb{R}^{n}\right)$ também não é díficil de provar. 
Portanto $T$ aplica $Q$ em $Q$.

Tomemos $t_{0} \leq s_{1}<s_{2} \leq t_{0}+\Delta$ e $z_{1}, z_{2} \in Q$. Usando as condições $(B)$ e $\left(B^{\prime}\right)$, obtemos

$$
\begin{gathered}
\left|T z_{2}\left(s_{2}\right)-T z_{1}\left(s_{2}\right)-\left[T z_{2}\left(s_{1}\right)-T z_{1}\left(s_{1}\right)\right]\right|= \\
=\left|\int_{s_{1}}^{s_{2}}\left[f\left(z_{2}, t\right)-f\left(z_{1}, t\right)\right] d t+\sum_{s_{1} \leq t_{j}<s_{2}}\left[I_{j}\left(z_{2}\left(t_{j}\right)\right)-I_{j}\left(z_{1}\left(t_{j}\right)\right)\right]\right| \leq \\
\leq \int_{s_{1}}^{s_{2}} L(t)\left|z_{2}(t)-z_{1}(t)\right| d t+K_{2} \sum_{j=0}^{m}\left|z_{2}\left(t_{j}\right)-z_{1}\left(t_{j}\right)\right|\left[H_{t_{j}}\left(s_{2}\right)-H_{t_{j}}\left(s_{1}\right)\right] \leq \\
\leq \sup _{s \in\left[t_{0}, t_{0}+\Delta\right]}\left|z_{2}(s)-z_{1}(s)\right|\left(\int_{s_{1}}^{s_{2}} L(t) d t+K_{2} \sum_{j=0}^{m}\left[H_{t_{j}}\left(s_{2}\right)-H_{t_{j}}\left(s_{1}\right)\right]\right) \leq \\
\leq\left\|z_{2}-z_{1}\right\|_{B V\left(\left[t_{0}, t_{0}+\Delta\right]\right)}\left[h\left(s_{2}\right)-h\left(s_{1}\right)\right] .
\end{gathered}
$$

Como $\|z\|_{B V\left(\left[t_{0}, t_{0}+\Delta\right]\right)}=\left\|z\left(t_{0}\right)\right\|+\operatorname{var}_{t_{0}}^{t_{0}+\Delta}(z)$ define uma norma em $B V\left(\left[t_{0}, t_{0}+\Delta\right], \mathbb{R}^{n}\right)$, onde $\operatorname{var}_{t_{0}}^{t_{0}+\Delta}(z)$ denota a variação de $z$ no intervalo $\left[t_{0}, t_{0}+\Delta\right]$, temos

$$
\begin{aligned}
\left\|T z_{2}-T z_{1}\right\|_{B V\left(\left[t_{0}, t_{0}+\Delta\right]\right)} & \leq\left\|z_{2}-z_{1}\right\|_{B V\left(\left[t_{0}, t_{0}+\Delta\right]\right)}\left[h\left(t_{0}+\Delta\right)-h\left(t_{0}\right)\right] \\
& <\frac{1}{2}\left\|z_{2}-z_{1}\right\|_{B V\left(\left[t_{0}, t_{0}+\Delta\right]\right)}
\end{aligned}
$$

donde concluímos que T é uma contração. Assim, pelo teorema do ponto fixo de Banach, o resultado segue.

Agora, consideremos o caso em que $t_{0}$ não é um ponto de continuidade de $h$ (ou de $\left.h_{2}\right)$. Definamos

$$
\bar{h}_{2}(t)=\left\{\begin{array}{l}
h_{2}(t), \quad t=t_{0} \\
h_{2}(t)-h_{2}\left(t_{0}+\right)+h_{2}\left(t_{0}\right)=h_{2}(t)-h_{2}\left(t_{0}+\right), \quad t_{0} \leq t \leq t_{0}+\sigma
\end{array}\right.
$$

e $\bar{h}=h_{1}+\bar{h}_{2}$. Então $\bar{h}$ é contínua em $t=t_{0}$, contínua à esquerda e não decrescente.

Definamos um operador de impulso $\bar{I}_{0}$ de $\mathbb{R}^{n}$ em $\mathbb{R}^{n}$ tal que

$$
\bar{I}_{0}(y(t))=\left\{\begin{array}{l}
I_{0}(y(t)), \quad t=t_{0} \\
I_{0}(y(t))-I_{0}\left(y\left(t_{0}+\right)\right)+I_{0}\left(y\left(t_{0}\right)\right), \quad t_{0} \leq t \leq t_{0}+\sigma .
\end{array}\right.
$$


sempre que $y \in G^{-}\left(\left[t_{0}, t_{0}+\sigma\right], \mathbb{R}^{n}\right)$, e consideremos o sistema diferencial impulsivo auxiliar

$$
\left\{\begin{array}{l}
\dot{z}(t)=f\left(z_{t}, t\right), t \neq t_{k} \\
\Delta z\left(t_{k}\right)=I_{k}\left(z\left(t_{k}\right)\right), k=1, \ldots, m \\
\Delta z\left(t_{0}\right)=\bar{I}_{0}\left(z\left(t_{0}\right)\right) \\
z_{t_{0}}=\bar{\phi}
\end{array}\right.
$$

onde

$$
\bar{\phi}(\theta)=\left\{\begin{array}{l}
\phi(\theta),-r \leq \theta<0 \\
y\left(t_{0}+\right), \theta=0
\end{array}\right.
$$

Então, para $s \in\left[t_{0}, t_{0}+\sigma\right]$, temos

$$
\left|\int_{t_{0}}^{s} f\left(z_{t}, t\right) d t+\bar{I}_{0}\left(z\left(t_{0}\right)\right)+\sum_{t_{0}<t_{j}<s} I_{j}\left(z\left(t_{j}\right)\right)\right| \leq \bar{h}(s)-\bar{h}\left(t_{0}\right) .
$$

Como no caso anterior, seja $\Delta>0$ tal que $\left[t_{0}, t_{0}+\Delta\right] \subset\left[t_{0}, t_{0}+\sigma\right)$ e $\bar{h}\left(t_{0}+\Delta\right)-\bar{h}\left(t_{0}\right)<\frac{1}{2}$. Então, definimos $\bar{Q}$ como o conjunto das funções $z:\left[t_{0}, t_{0}+\Delta\right] \rightarrow \mathbb{R}^{n}$ tal que $z \in$ $B V\left(\left[t_{0}, t_{0}+\Delta\right], \mathbb{R}^{n}\right)$ e $\left|z(t)-y\left(t_{0}+\right)\right| \leq \bar{h}(t)-\bar{h}\left(t_{0}\right)$ para $t \in\left[t_{0}, t_{0}+\Delta\right]$ e consideramos o operador $\bar{T}$ definido em $\bar{Q}$ dado por

$$
\bar{T} z(s)=y\left(t_{0}+\right)+\int_{t_{0}}^{s} f\left(z_{t}, t\right) d t+\bar{I}_{0}\left(z\left(t_{0}\right)\right)+\sum_{t_{0}<t_{j}<s} I_{j}\left(z\left(t_{j}\right)\right) .
$$

Seguindo o procedimento do caso anterior, é possível mostrar que a equação (2.16) admite uma única solução $z(t)$ em $\left[t_{0}, t_{0}+\Delta\right]$. Então, definindo $y_{t_{0}}=\phi$ e $y(t)=z(t)$, para $t>t_{0}$, obtemos uma solução única de (2.13) em $\left[t_{0}, t_{0}+\Delta\right]$ para a qual $y_{t_{0}}=\phi$.

O resultado que segue é inédito para a teoria de EDFRIs. Na ausência de impulsos, tal resultado também é novo na teoria de EDFRs.

Consideraremos a seguinte sequência de problemas de valor inicial:

$$
\left\{\begin{array}{l}
\dot{y}(t)=f_{p}\left(y_{t}, t\right), t \neq t_{k} \\
\Delta y\left(t_{k}\right)=I_{k}^{p}\left(y\left(t_{k}\right)\right), k=0,1, \ldots, m \\
y_{t_{0}}=\phi_{p}, \mathrm{p}=1,2, \ldots
\end{array}\right.
$$

onde $t_{0}<t_{1}<\ldots<t_{k}<\ldots<t_{m} \leq t_{0}+\sigma$, e para cada $p=1,2, \ldots, x \rightarrow I_{k}^{p}(x)$ aplica 
$\mathbb{R}^{n}$ nele mesmo e

$$
\Delta y\left(t_{k}\right):=y\left(t_{k}+\right)-y\left(t_{k}-\right)=y\left(t_{k}+\right)-y\left(t_{k}\right), \quad k=0,1,2, \ldots, m
$$

Mostraremos que, nas condições $(A),(B),\left(A^{\prime}\right)$ e $\left(B^{\prime}\right)$ para todos os $f_{p}$ e $I_{k}^{p}$, com $k=0,1,2, \ldots, m$ e $p=1,2, \ldots$, a sequência $\left\{y_{p}\right\}_{p \geq 1}$ de soluções de (2.17) será equilimitada e uniformemente de variação limitada em $\left[t_{0}, t_{0}+\sigma\right]$.

Teorema 2.29. Suponhamos que $\phi_{p} \in G^{-}\left([-r, 0], \mathbb{R}^{n}\right)$, para $p=0,1, \ldots$ Suponhamos, também que, para $p=0,1, \ldots$, as funções $f_{p}: G^{-}\left([-r, 0], \mathbb{R}^{n}\right) \times\left[t_{0}, t_{0}+\sigma\right] \rightarrow \mathbb{R}^{n} e$ $I_{k}^{p}: \mathbb{R}^{n} \rightarrow \mathbb{R}^{n}, k=0,1, \ldots, m$, satisfaçam as condições $(A),(B),\left(A^{\prime}\right)$ e $\left(B^{\prime}\right)$ para as mesmas funções $M$ e $L$ e as mesmas constantes $K_{1}$ e $K_{2}$. Então existirá um $\Delta>0$ tal que $y_{p}:\left[t_{0}-r, t_{0}+\Delta\right] \rightarrow \mathbb{R}^{n}$ será uma solução de $(2.17)$, para cada $p$, e a sequência $\left\{y_{p}\right\}_{p \geq 1}$ será equilimitada e uniformemente de variação limitada em $\left[t_{0}, t_{0}+\Delta\right]$.

Prova: Pela prova do Teorema 2.28, fica claro que podemos obter um único $\Delta>0$ tal que a solução $y_{p}:\left[t_{0}-r, t_{0}+\Delta\right] \rightarrow \mathbb{R}^{n}$ de (2.17) existe e é única, independentemente de $p$. Portanto, para $p=0,1, \ldots$, temos

$$
y_{p}(s)=y_{p}\left(t_{0}\right)+\int_{t_{0}}^{s} f_{p}\left(\left(y_{p}\right)_{t}, t\right) d t+\sum_{t_{0} \leq t_{j}<s} I_{j}^{p}\left(y_{p}\left(t_{j}\right)\right), \quad s \in\left[t_{0}, t_{0}+\Delta\right] .
$$

Consideremos uma partição $t_{0}=s_{0}<s_{1}<\ldots<s_{n}=t_{0}+\Delta$ de $\left[t_{0}, t_{0}+\Delta\right]$. Então

$$
y_{p}\left(s_{i}\right)-y_{p}\left(s_{i-1}\right)=\int_{s_{i-1}}^{s_{i}} f_{p}\left(\left(y_{p}\right)_{t}, t\right) d t+\sum_{s_{i-1} \leq t_{j}<s_{i}} I_{j}\left(y_{p}\left(t_{j}\right)\right), \quad i=1,2, \ldots, n .
$$

Portanto, as condições $(A)$ e $\left(A^{\prime}\right)$ implicam

$$
\begin{aligned}
\sum_{i=1}^{n}\left|y_{p}\left(s_{i}\right)-y_{p}\left(s_{i-1}\right)\right| & \leq \sum_{i=1}^{n}\left|\int_{s_{i-1}}^{s_{i}} f_{p}\left(\left(y_{p}\right)_{t}, t\right) d t\right|+\sum_{t_{0} \leq t_{j}<t_{0}+\Delta}\left|I_{j}\left(y_{p}\left(t_{j}\right)\right)\right| \\
& \leq \int_{t_{0}}^{t_{0}+\Delta} M(s) d s+K_{1} l
\end{aligned}
$$

onde $l$ é o número dos momentos de impulso no intervalo $\left[t_{0}, t_{0}+\Delta\right]$. Assim,

$$
\operatorname{var}_{t_{0}}^{t_{0}+\Delta}\left(y_{p}\right) \leq \int_{t_{0}}^{t_{0}+\Delta} M(s) d s+K_{1} l, \quad \text { para todo } p \in \mathbb{N}
$$

e, portanto, $\left\{y_{p}\right\}_{p \geq 1}$ é uniformemente de variação limitada em $\left[t_{0}, t_{0}+\Delta\right]$. 
Agora, iremos mostrar que $\left\{y_{p}\right\}_{p \geq 1}$ é equilimitada. Novamente, como

$$
y_{p}(t)=y_{p}\left(t_{0}\right)+\int_{t_{0}}^{t} f\left(\left(y_{p}\right)_{s}, s\right) d s+\sum_{t_{0} \leq t_{j}<t} I_{j}\left(y_{p}\left(t_{j}\right)\right),
$$

então, para cada $t \in\left[t_{0}, t_{0}+\Delta\right]$ e cada $p=1,2,3, \ldots$, temos

$$
\begin{aligned}
\left|y_{p}(t)\right| & \leq\left|y_{p}\left(t_{0}\right)\right|+\left|\int_{t_{0}}^{t} f_{p}\left(\left(y_{p}\right)_{s}, s\right) d s\right|+\left|\sum_{t_{0} \leq t_{j}<t_{0}+\Delta} I_{j}\left(y_{p}\left(t_{j}\right)\right)\right| \\
& \leq\left|y_{p}\left(t_{0}\right)\right|+\int_{t_{0}}^{t} M(s) d s+K_{1} l \\
& \leq\left|y_{p}\left(t_{0}\right)\right|+\int_{t_{0}}^{t_{0}+\sigma} M(s) d s+K_{1} l
\end{aligned}
$$

Portanto $\left\{y_{p}\right\}_{p \geq 1}$ é equilimitada em $\left[t_{0}, t_{0}+\Delta\right]$ e o resultado segue.

Em geral, não podemos esperar que uma solução de uma EDFRI dependa dos valores iniciais. Mencionamos [29] para uma discussão elucidativa sobre dependência contínua de soluções de uma EDFRI cujos operadores de impulsos também envolvem retardos.

O próximo teorema é um resultado sobre dependência contínua. Tal resultado junto com o Teorema 2.29 são importantes para provar o teorema que virá a seguir.

Não demonstraremos o próximo resultado agora, pois para a demonstração deste, precisaremos de dois teoremas que serão apresentados no capítulo seguinte. Devido a isso, apenas o enunciaremos e, no próximo capítulo, apresentaremos sua prova.

Teorema 2.30 ([7], Teorema 4.1). Suponhamos que, para cada $p=0,1, \ldots, \phi_{p} \in$ $G^{-}\left([-r, 0], \mathbb{R}^{n}\right)$ e as funções $f_{p}: G^{-}\left([-r, 0], \mathbb{R}^{n}\right) \times\left[t_{0}, t_{0}+\sigma\right] \rightarrow \mathbb{R}^{n}$ e $I_{k}^{p}: \mathbb{R}^{n} \rightarrow \mathbb{R}^{n}$, $k=0,1,2, \ldots$, satisfaçam as condições $(A),(B),\left(A^{\prime}\right)$ e $\left(B^{\prime}\right)$ para as mesmas funções $M$ e $L$ e as mesmas constantes $K_{1}$ e $K_{2}$. Consideremos que as relações

$$
\lim _{p \rightarrow \infty} \sup _{\vartheta \in\left[t_{0}, t_{0}+\sigma\right]}\left|\int_{t_{0}}^{\vartheta}\left[f_{p}\left(y_{s}, s\right)-f_{0}\left(y_{s}, s\right)\right] d s\right|=0
$$

para todo $y \in P C_{1} e$

$$
\lim _{p \rightarrow \infty} I_{k}^{p}(x)=I_{k}^{0}(x)
$$

para todo $x \in \mathbb{R}^{n}$ e $k=0,1, \ldots, m$ estejam satisfeitas. Suponhamos, ainda, que $y_{p}$ : 
$\left[t_{0}-r, t_{0}+\sigma\right] \rightarrow \mathbb{R}^{n}, p=1,2, \ldots$, seja uma solução em $\left[t_{0}-r, t_{0}+\sigma\right]$ do problema

$$
\left\{\begin{array}{l}
\dot{y}(t)=f_{p}\left(y_{t}, t\right), t \neq t_{k} \\
\Delta y\left(t_{k}\right)=I_{k}^{p}\left(y\left(t_{k}\right)\right), k=0,1, \ldots, m \\
y_{t_{0}}=\phi_{p}
\end{array}\right.
$$

tal que

$$
\lim _{p \rightarrow \infty} y_{p}(s)=y(s) \text { uniformemente em }\left[t_{0}-r, t_{0}+\sigma\right] .
$$

Então $y:\left[t_{0}-r, t_{0}+\sigma\right] \rightarrow \mathbb{R}^{n}$ será uma solução em $\left[t_{0}-r, t_{0}+\sigma\right]$ do problema

$$
\left\{\begin{array}{l}
\dot{y}(t)=f_{0}\left(y_{t}, t\right), t \neq t_{k} \\
\Delta y\left(t_{k}\right)=I_{k}^{0}\left(y\left(t_{k}\right)\right), k=0,1, \ldots, m \\
y_{t_{0}}=\phi_{0}
\end{array}\right.
$$

As hipóteses (2.19) e (2.20) no Teorema 2.29 asseguram que, se a sequência $\left\{y_{p}\right\}_{p \geq 1}$, $y_{p}:\left[t_{0}-r, t_{0}+\Delta\right] \rightarrow \mathbb{R}^{n}, p=1,2, \ldots$, de soluções de (2.21) convergir uniformemente para uma função $y:\left[t_{0}-r, t_{0}+\Delta\right] \rightarrow \mathbb{R}^{n}$, então este limite será uma solução de (2.23).

O próximo resultado também é inédito e diz que se adicionarmos uma condição de unicidade para a equação "limite", então para $p \in \mathbb{N}$ suficientemente grande, $y_{p}:\left[t_{0}-\right.$ $\left.r, t_{0}+\Delta\right] \rightarrow \mathbb{R}^{n}$ será uma solução de $(2.21)$, desde que a sequência dos valores iniciais $\left\{\phi_{p}\right\}_{p \geq 1}$ convirja uniformemente em $[-r, 0]$.

Teorema 2.31. Suponhamos que, para cada $p=0,1,2, \ldots, f_{p}(\phi, t): G^{-}\left([-r, 0], \mathbb{R}^{n}\right) \times$ $\left[t_{0}, t_{0}+\sigma\right] \rightarrow \mathbb{R}^{n}$ satisfaçam as condições $(A)$ e $(B)$ para as mesmas funções $M$ e $L$ e, para cada $p=0,1,2, \ldots$, e cada $k=0,1,2, \ldots, I_{k}{ }^{p}: \mathbb{R}^{n} \rightarrow \mathbb{R}^{n}$ satisfaçam as condições $\left(A^{\prime}\right)$ e $\left(B^{\prime}\right)$ para as mesmas constantes $K_{1}$ e $K_{2}$. Suponhamos, também, que

$$
\lim _{p \rightarrow \infty} \int_{t_{0}}^{t}\left[f_{p}\left(y_{s}, s\right)-f_{0}\left(y_{s}, s\right)\right] d s=0, \quad t \in\left[t_{0}, t_{0}+\sigma\right]
$$

para todo $y \in P C_{1}$, e

$$
\lim _{p \rightarrow \infty} I_{k}^{p}(x)=I_{k}^{0}(x)
$$

para todo $x \in \mathbb{R}^{n}$ e $k=0,1, \ldots, m$. Seja $y:\left[t_{0}-r, t_{0}+\sigma\right] \rightarrow \mathbb{R}^{n}$ uma solução de

$$
\left\{\begin{array}{l}
\dot{y}(t)=f_{0}\left(y_{t}, t\right), t \neq t_{k} \\
\Delta y\left(t_{k}\right)=I_{k}^{0}\left(y\left(t_{k}\right)\right), k=0,1, \ldots, m \\
y_{t_{0}}=\phi_{0}
\end{array}\right.
$$


em $\left[t_{0}-r, t_{0}+\sigma\right]$. Suponhamos que se existir $\rho>0$ tal que

$$
\sup _{\theta \in[-r, 0]}\left|u(\theta)-\phi_{0}(\theta)\right|<\rho
$$

então $u \in G^{-}\left([-r, 0], \mathbb{R}^{n}\right)$. Suponhamos, ainda mais, que $\phi_{p} \rightarrow \phi_{0}$ uniformemente em $[-r, 0]$, quando $p \rightarrow \infty$. Então, para $p \in \mathbb{N}$ suficientemente grande, existirá uma solução $y_{p} d e$

$$
\left\{\begin{array}{l}
\dot{y}(t)=f_{p}\left(y_{t}, t\right), t \neq t_{k} \\
\Delta y\left(t_{k}\right)=I_{k}^{p}\left(y\left(t_{k}\right)\right), k=0,1, \ldots, m \\
y_{t_{0}}=\phi_{p}
\end{array}\right.
$$

$e m\left[t_{0}-r, t_{0}+\sigma\right] e$

$$
\lim _{p \rightarrow \infty} y_{p}(s)=y(s), \quad s \in\left[t_{0}-r, t_{0}+\sigma\right]
$$

Prova: Esta demonstração foi inspirada na prova do Teorema 8.6 de [26] para EDOGs. Usaremos fortemente o fato de que as funções $f_{p}, p=0,1,2, \ldots$, assumem valores em um espaço de dimensão finita para podermos aplicar o Princípio da Escolha de Helly.

Como $\phi_{p} \rightarrow \phi_{0}$ uniformemente em $[-r, 0]$ quando $p \rightarrow \infty$, existem $\rho>0$ e $k \in \mathbb{N}$ tais que

$$
\sup _{\theta \in[-r, 0]}\left|\phi_{p}(\theta)-\phi_{0}(\theta)\right|<\rho, \quad \text { para } p>k .
$$

Portanto, por hipótese, $\phi_{p} \in G^{-}\left([-r, 0], \mathbb{R}^{n}\right)$, para $p>k$. Logo, pelo Teorema de Existência (Teorema 2.28), para $p>k$, existe um $\Delta>0$ tal que $y_{p}$ é uma solução de (2.27) em $\left[t_{0}-r, t_{0}+\Delta\right]$.

Afirmamos que

$$
\lim _{p \rightarrow \infty} y_{p}(s)=y(s), \quad \text { para } s \in\left[t_{0}-r, t_{0}+\sigma\right]
$$

De fato, pelo Teorema 2.30, se a sequência $\left\{y_{p}\right\}_{p \geq 1}$ admitir uma subsequência convergente, então como $\phi_{p} \rightarrow \phi_{0}$, seguirá da unicidade de soluções que existe um $\Delta>0$ tal que $\lim _{p \rightarrow \infty} y_{p}(s)=y(s)$, para $s \in\left[t_{0}-r, t_{0}+\Delta\right]$, onde $y_{t_{0}}=\phi_{0}$.

Usaremos o Princípio da Escolha de Helly para provar o fato de que $\left\{y_{p}\right\}_{p \geq 1}$ admite uma subsequência convergente.

Pelo Teorema 2.29, a sequência $\left\{y_{p}\right\}, p>k$, de funções em $\left[t_{0}, t_{0}+\Delta\right]$ é equilimitada e uniformemente de variação limitada. Portanto, pelo Princípio da Escolha de Helly, a sequência $\left\{y_{p}\right\}, p>k$, contém uma subsequência pontualmente convergente e, portanto, $y(t)$ é o único ponto de acumulação da sequência $y_{p}(t)$ para todo $t \in\left[t_{0}, t_{0}+\Delta\right]$. Logo, o 
teorema vale em $\left[t_{0}, t_{0}+\Delta\right]$. O teorema também vale em $\left[t_{0}-r, t_{0}\right]$, já que $\phi_{p} \rightarrow \phi_{0}$.

Agora, vamos assumir que o resultado de convergência não acontece em todo o intervalo $\left[t_{0}-r, t_{0}+\sigma\right]$. Então, existe um $\Delta^{\prime}, 0<\Delta^{\prime}<\sigma$, tal que para todo $\Delta<\Delta^{\prime}$ e para $p>k$, existe uma solução $y_{p}$ de $(2.27)$ em $\left[t_{0}-r, t_{0}+\Delta\right]$, com $\left(y_{p}\right)_{t_{0}}=\phi_{p}$, e $\lim _{p \rightarrow \infty} y_{p}(t)=y(t)$ para $t \in\left[t_{0}-r, t_{0}+\Delta\right]$, mas isto não acontece em $\left[t_{0}-r, t_{0}+\Delta\right]$, para $\Delta>\Delta^{\prime}$.

Pela prova do Teorema 2.28, temos

$$
\left|y_{k}\left(s_{2}\right)-y_{k}\left(s_{1}\right)\right|<\left|h\left(s_{2}\right)-h\left(s_{1}\right)\right|
$$

para todo $s_{2}, s_{1} \in\left[t_{0}-r, t_{0}+\Delta^{\prime}\right)$ e todo $p>k$. Portanto os limites

$$
y_{p}\left(\left(t_{0}+\Delta^{\prime}\right)-\right)=\lim _{\varepsilon \rightarrow 0^{-}} y_{p}\left(t_{0}+\Delta^{\prime}+\varepsilon\right), \quad p>k
$$

existem e $y_{p}\left(\left(t_{0}+\Delta^{\prime}\right)-\right)=y\left(t_{0}+\Delta^{\prime}\right)$, para $p>k$, já que $y$ é contínua à esquerda.

Definindo $y_{p}\left(t_{0}+\Delta^{\prime}\right)=y_{p}\left(\left(t_{0}+\Delta^{\prime}\right)-\right)$, para $p>k$, então $\lim _{p \rightarrow \infty} y_{p}\left(t_{0}+\Delta^{\prime}\right)=y\left(t_{0}+\Delta^{\prime}\right)$. Portanto o teorema acontece em $\left[t_{0}-r, t_{0}+\Delta^{\prime}\right]$. Daí, usando $t_{0}+\Delta^{\prime}<t_{0}+\sigma$ como ponto inicial, podemos provar analogamente que o teorema acontece no intervalo $\left[t_{0}+\Delta^{\prime}, t_{0}+\right.$ $\Delta^{\prime}+\eta$ ], para algum $\eta>0$, e isto contradiz nossa hipótese. Logo, o teorema acontece em todo o intervalo $\left[t_{0}-r, t_{0}+\sigma\right]$. 


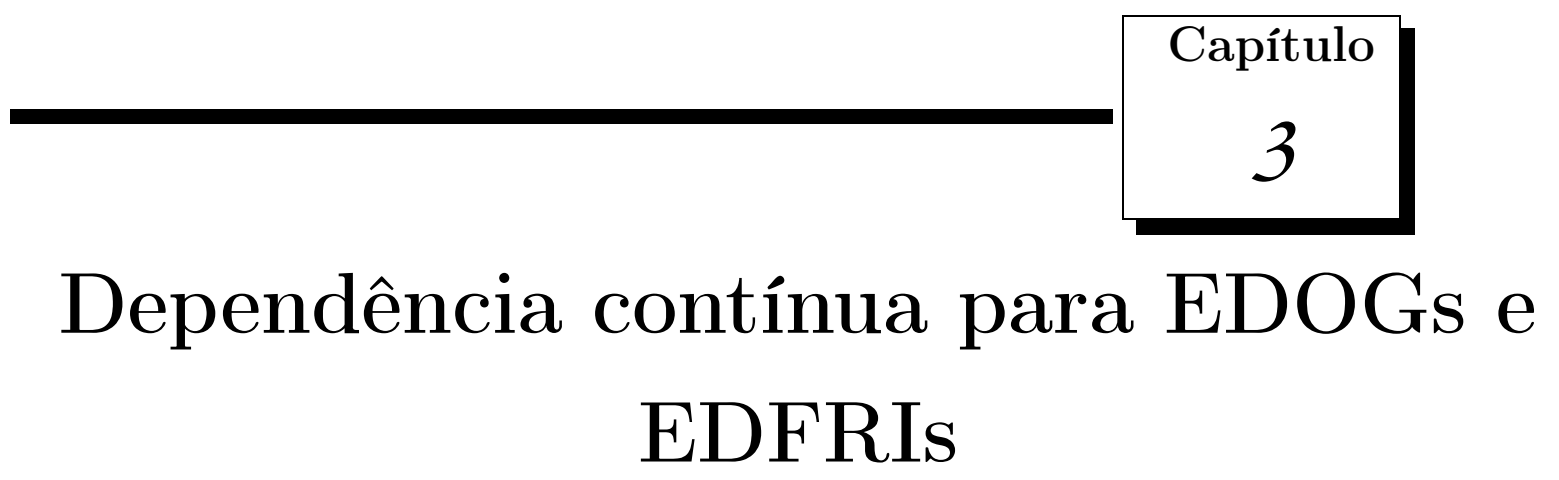

Este capítulo está dividido em duas seções. Na primeira delas, trataremos da correspondência entre a solução de uma equação diferencial funcional retardada impulsiva (EDFRI) e a solução de uma equação diferencial ordinária generalizada (EDOG). Na segunda seção, abordaremos a dependência contínua de soluções de certas EDOGs, a partir de EDFRIs e vice-versa.

As principais referências para este capítulo são [7] e [26].

\subsection{Correspondência entre EDFRIs e EDOGs}

Começaremos esta seção, definindo algumas funções que usaremos adiante.

Para $y \in P C_{1}$ e $t \in\left[t_{0}-r, t_{0}+\sigma\right]$, definamos

$$
F(y, t)(\vartheta)=\left\{\begin{array}{l}
0, \quad t_{0}-r \leq \vartheta \leq t_{0} \text { ou } t_{0}-r \leq t \leq t_{0} \\
\int_{t_{0}}^{\vartheta} f\left(y_{s}, s\right) d s, \quad t_{0} \leq \vartheta \leq t \leq t_{0}+\sigma \\
\int_{t_{0}}^{t} f\left(y_{s}, s\right) d s, \quad t_{0} \leq t \leq \vartheta \leq t_{0}+\sigma
\end{array}\right.
$$

Então para cada $(y, t) \in P C_{1} \times\left[t_{0}-r, t_{0}+\sigma\right]$, a equação (3.1) define um elemento $F(y, t)$ de $C\left(\left[t_{0}-r, t_{0}+\sigma\right], \mathbb{R}^{n}\right)$ e

$$
F: P C_{1} \times\left[t_{0}-r, t_{0}+\sigma\right] \rightarrow C\left(\left[t_{0}-r, t_{0}+\sigma\right], \mathbb{R}^{n}\right)
$$


ou seja, $F(y, t)(\tau) \in \mathbb{R}^{n}$ é o valor de $F(y, t)$ em um ponto $\tau \in\left[t_{0}-r, t_{0}+\sigma\right]$, onde $C\left([a, b], \mathbb{R}^{n}\right)$ denota o espaço de Banach das funções contínuas que aplicam $[a, b]$ em $\mathbb{R}^{n}$ com a norma usual do supremo.

Suponhamos que as condições (A) e (B) do capítulo anterior (página 68) estejam satisfeitas para a aplicação $f(\phi, t): G^{-}\left([-r, 0], \mathbb{R}^{n}\right) \times\left[t_{0}, t_{0}+\sigma\right] \rightarrow \mathbb{R}^{n}$.

Dados $x \in P C_{1}$ e $t_{0} \leq s_{1}<s_{2}<t_{0}+\sigma$, temos para $F: P C_{1} \times\left[t_{0}-r, t_{0}+\sigma\right] \rightarrow$ $C\left(\left[t_{0}-r, t_{0}+\sigma\right], \mathbb{R}^{n}\right)$ dado por (3.1) a seguinte relação:

$$
F\left(x, s_{2}\right)(\vartheta)-F\left(x, s_{1}\right)(\vartheta)=\left\{\begin{array}{l}
0, \vartheta \in\left[t_{0}-r, s_{1}\right] \\
\int_{s_{1}}^{\vartheta} f\left(x_{s}, s\right) d s, \quad \vartheta \in\left[s_{1}, s_{2}\right] \\
\int_{s_{1}}^{s_{2}} f\left(x_{s}, s\right) d s, \quad \vartheta \in\left[s_{2}, t_{0}+\sigma\right] .
\end{array}\right.
$$

Portanto, para um $x \in P C_{1}$ e para $t_{0} \leq s_{1}<s_{2}<t_{0}+\sigma$, a condição (A) implica que

$$
\begin{gathered}
\left\|F\left(x, s_{2}\right)-F\left(x, s_{1}\right)\right\|=\sup _{\vartheta \in\left[t_{0}-r, t_{0}+\sigma\right]}\left|F\left(x, s_{2}\right)(\vartheta)-F\left(x, s_{1}\right)(\vartheta)\right|= \\
=\sup _{\vartheta \in\left[s_{1}, s_{2}\right]}\left|F\left(x, s_{2}\right)(\vartheta)-F\left(x, s_{1}\right)(\vartheta)\right|=\sup _{\vartheta \in\left[s_{1}, s_{2}\right]}\left|\int_{s_{1}}^{\vartheta} f\left(x_{s}, s\right) d s\right| \leq \\
\leq \sup _{\vartheta \in\left[s_{1}, s_{2}\right]} \int_{s_{1}}^{\vartheta} M(s) d s=\int_{s_{1}}^{s_{2}} M(s) d s .
\end{gathered}
$$

Similarmente, usando (3.2) e (B), se $x, y \in P C_{1}$ e $t_{0} \leq s_{1}<s_{2}<t_{0}+\sigma$, então teremos

$$
\begin{gathered}
\left\|F\left(x, s_{2}\right)-F\left(x, s_{1}\right)-F\left(y, s_{2}\right)+F\left(y, s_{1}\right)\right\|= \\
=\sup _{\vartheta \in\left[s_{1}, s_{2}\right]}\left|\int_{s_{1}}^{\vartheta}\left[f\left(x_{s}, s\right)-f\left(y_{s}, s\right) d s\right]\right| \leq \int_{s_{1}}^{s_{2}} L(s)\left\|x_{s}-y_{s}\right\| d s \leq \\
\leq \sup _{\vartheta \in\left[s_{1}-r, s_{2}\right]}|x(\vartheta)-y(\vartheta)| \int_{s_{1}}^{s_{2}} L(s) d s \leq\|x-y\| \int_{s_{1}}^{s_{2}} L(s) d s .
\end{gathered}
$$

Definamos $h_{1}:\left[t_{0}-r, t_{0}+\sigma\right] \rightarrow \mathbb{R}$ por

$$
h_{1}(t)=\left\{\begin{array}{l}
\int_{t_{0}}^{t}[M(s)+L(s)] d s, t \in\left[t_{0}, t_{0}+\sigma\right], \\
0, t \in\left[t_{0}-r, t_{0}\right] .
\end{array}\right.
$$

Então a função $h_{1}$ é (absolutamente) contínua e não decrescente, pois $M, L:\left[t_{0}, t_{0}+\sigma\right] \rightarrow$ 
$\mathbb{R}$ são funções não negativas Lebesgue integráveis.

Assim, de acordo com as relações (3.3) e (3.4), temos

$$
\left\|F\left(x, s_{2}\right)-F\left(x, s_{1}\right)\right\| \leq\left|h_{1}\left(s_{2}\right)-h_{1}\left(s_{1}\right)\right|
$$

para quaisquer $\left(x, s_{2}\right),\left(x, s_{1}\right) \in P C_{1} \times\left[t_{0}, t_{0}+\sigma\right] \mathrm{e}$

$$
\left\|F\left(x, s_{2}\right)-F\left(x, s_{1}\right)-F\left(y, s_{2}\right)+F\left(y, s_{1}\right)\right\| \leq\|x-y\|\left|h_{1}\left(s_{2}\right)-h_{1}\left(s_{1}\right)\right|
$$

para quaisquer $\left(x, s_{2}\right),\left(x, s_{1}\right),\left(y, s_{2}\right),\left(y, s_{1}\right) \in P C_{1} \times\left[t_{0}, t_{0}+\sigma\right]$. Portanto $F \in \mathcal{F}\left(\Omega, h_{1}\right)$, onde $\Omega=P C_{1} \times\left[t_{0}-r, t_{0}+\sigma\right]$.

Agora, vamos considerar os termos impulsivos do problema (2.13).

Para $y \in P C_{1}$ e $t \in\left[t_{0}-r, t_{0}+\sigma\right]$, definamos

$$
J(y, t)(\vartheta)=\sum_{k=0}^{m} H_{t_{k}}(t) H_{t_{k}}(\vartheta) I_{k}\left(y\left(t_{k}\right)\right)
$$

para $\vartheta \in\left[t_{0}-r, t_{0}+\sigma\right]$, onde $H_{t_{k}}$ é a função Heaviside contínua à esquerda concentrada em $t_{k}$. Vamos considerar que as condições $\left(A^{\prime}\right)$ e $\left(B^{\prime}\right)$ do capítulo anterior estejam satisfeitas. Então, para $y \in P C_{1}$ e $t_{0}-r \leq s_{1} \leq s_{2} \leq t_{0}+\sigma$, temos

$$
J\left(y, s_{2}\right)(\vartheta)-J\left(y, s_{1}\right)(\vartheta)=\sum_{k=0}^{m}\left[H_{t_{k}}\left(s_{2}\right)-H_{t_{k}}\left(s_{1}\right)\right] H_{t_{k}}(\vartheta) I_{k}\left(y\left(t_{k}\right)\right)
$$

onde $\vartheta \in\left[t_{0}-r, t_{0}+\sigma\right]$. Logo, para $t_{0}-r \leq s_{1}<s_{2} \leq t_{0}+\sigma$ e $y \in P C_{1}$, temos

$$
\begin{gathered}
\left\|J\left(x, s_{2}\right)-J\left(x, s_{1}\right)\right\|=\sup _{\vartheta \in\left[t_{0}-r, t_{0}+\sigma\right]} \sum_{k=0}^{m}\left[H_{t_{k}}\left(s_{2}\right)-H_{t_{k}}\left(s_{1}\right)\right] H_{t_{k}}(\vartheta)\left|I_{k}\left(x\left(t_{k}\right)\right)\right| \leq \\
\leq \sum_{k=0}^{m}\left[H_{t_{k}}\left(s_{2}\right)-H_{t_{k}}\left(s_{1}\right)\right] K_{1}
\end{gathered}
$$

e, similarmemte, se $x, y \in P C_{1}$ e $t_{0}-r \leq s_{1} \leq s_{2} \leq t_{0}+\sigma$, temos

$$
\left\|J\left(x, s_{2}\right)-J\left(x, s_{1}\right)-J\left(y, s_{2}\right)+J\left(x, s_{1}\right)\right\| \leq \sum_{k=0}^{m}\left[H_{t_{k}}\left(s_{2}\right)-H_{t_{k}}\left(s_{1}\right)\right] K_{2}\|x-y\| .
$$

Definamos $h_{2}:\left[t_{0}-r, t_{0}+\sigma\right] \rightarrow \mathbb{R}$ por 


$$
h_{2}(t)=\max \left(K_{1}, K_{2}\right) \sum_{k=0}^{m} H_{t_{k}}(t) .
$$

Então $h_{2}$ é contínua à esquerda e não decrescente. Além disso, por (3.10) e (1.22), temos

$$
\left\|J\left(x, s_{2}\right)-J\left(x, s_{1}\right)\right\| \leq h_{2}\left(s_{2}\right)-h_{2}\left(s_{1}\right)
$$

e

$$
\left\|J\left(x, s_{2}\right)-J\left(x, s_{1}\right)-J\left(y, s_{2}\right)+J\left(x, s_{1}\right)\right\| \leq\|x-y\|\left[h_{2}\left(s_{2}\right)-h_{2}\left(s_{1}\right)\right]
$$

desde que $x, y \in P C_{1}$ e $t_{0} \leq s_{1}<s_{2} \leq t_{0}+\sigma$. Logo $J \in \mathcal{F}\left(\Omega, h_{2}\right)$, onde $\Omega=P C_{1} \times\left[t_{0}-\right.$ $\left.r, t_{0}+\sigma\right]$.

Agora, consideremos $F(y, t)$ dado por $(3.1)$ e $J(y, t)$ dado por (3.8) e seja

$$
G(y, t)=F(y, t)+J(y, t)
$$

onde $y \in P C_{1}$ e $t \in\left[t_{0}-r, t_{0}+\sigma\right]$. Então $G(y, t)$ pertence a $G^{-}\left(\left[t_{0}-r, t_{0}+\sigma\right], \mathbb{R}^{n}\right)$, ou seja,

$$
G: P C_{1} \times\left[t_{0}-r, t_{0}+\sigma\right] \rightarrow G^{-}\left(\left[t_{0}-r, t_{0}+\sigma\right], \mathbb{R}^{n}\right) .
$$

Por (3.6) e (3.13), temos

$$
\begin{aligned}
\left\|G\left(x, s_{2}\right)-G\left(x, s_{1}\right)\right\| & \leq\left\|F\left(x, s_{2}\right)-F\left(x, s_{1}\right)\right\|+\left\|J\left(x, s_{2}\right)-J\left(x, s_{1}\right)\right\| \leq \\
& \leq h_{1}\left(s_{2}\right)-h_{1}\left(s_{1}\right)+h_{2}\left(s_{2}\right)-h_{2}\left(s_{1}\right)=h\left(s_{2}\right)-h\left(s_{1}\right)
\end{aligned}
$$

onde $h(t)=h_{1}(t)+h_{2}(t)$ é não decrescente e contínua à esquerda, $x \in P C_{1}$ e $t_{0} \leq s_{1}<$ $s_{2} \leq t_{0}+\sigma$.

Similarmente, (3.7) e (3.15) implicam

$$
\left\|G\left(x, s_{2}\right)-G\left(x, s_{1}\right)-G\left(y, s_{2}\right)+G\left(y, s_{1}\right)\right\| \leq\|x-y\|\left(h\left(s_{2}\right)-h\left(s_{1}\right)\right),
$$

para $x, y \in P C_{1}$ e $t_{0} \leq s_{1}<s_{2} \leq t_{0}+\sigma$. Logo, as desigualdades (3.16) e (3.17) mostram que $G \in \mathcal{F}(\Omega, h)$.

Consideraremos que $f$ e $I_{k}$ da equação (2.13) satisfazem as condições (A), (B), (A') e (B') e consideraremos a seguinte equação diferencial ordinária generalizada

$$
\frac{d x}{d \tau}=D G(x, t)
$$


onde a $G$ é dada por (3.15). Vamos estudar as relações entre a equação diferencial retardada impulsiva (2.13) e a equação diferencial ordinária generalizada (3.18).

Os resultados que apresentaremos abaixo foram extraídos de [7].

Lema 3.1 ([7], Lema 3.3). Seja $x(t)$ uma solução de (3.18) no intervalo $\left[t_{0}, t_{0}+\sigma\right]$, com $G$ dada por (3.15) e com a condição inicial $x\left(t_{0}\right) \in P C_{1}$ dada por $x\left(t_{0}\right)(\vartheta)=\phi(\vartheta)$ para $\vartheta \in\left[t_{0}-r, t_{0}\right]$ e $x\left(t_{0}\right)(\vartheta)=x\left(t_{0}\right)\left(t_{0}\right)$ para $\vartheta \in\left[t_{0}, t_{0}+\sigma\right]$. Então se $v \in\left[t_{0}, t_{0}+\sigma\right]$, teremos

$$
x(v)(\vartheta)=x(v)(v), \quad \vartheta \geq v, \vartheta \in\left[t_{0}-r, t_{0}+\sigma\right]
$$

$e$

$$
x(v)(\vartheta)=x(\vartheta)(\vartheta), \quad v \geq \vartheta, \vartheta \in\left[t_{0}-r, t_{0}+\sigma\right] .
$$

Prova: Suponhamos que $\vartheta \geq v$. Como $x$ é uma solução de (3.18), temos

$$
x(v)(v)=x\left(t_{0}\right)(v)+\int_{t_{0}}^{v} D G(x(\tau), t)(v)
$$

e similarmente,

$$
x(v)(\vartheta)=x\left(t_{0}\right)(\vartheta)+\int_{t_{0}}^{v} D G(x(\tau), t)(\vartheta) .
$$

Como $x\left(t_{0}\right)(\vartheta)=x\left(t_{0}\right)(v)$, pelas propriedades da condição inicial, temos

$$
x(v)(\vartheta)-x(v)(v)=\int_{t_{0}}^{v} D G(x(\tau), t)(\vartheta)-\int_{t_{0}}^{v} D G(x(\tau), t)(v) .
$$

Como a integral $\int_{t_{0}}^{v} D G(x(\tau), t)$ existe, para todo $\varepsilon>0$ existe um calibre $\delta$ em $\left[t_{0}, t_{0}+\sigma\right]$ tal que se $\left(\tau_{i},\left[s_{i-1}, s_{i}\right]\right)$ for uma divisão $\delta$-fina de $\left[t_{0}, v\right]$, então

$$
\left\|\sum_{i}\left[G\left(x\left(\tau_{i}\right), s_{i}\right)-G\left(x\left(\tau_{i}\right), s_{i-1}\right)\right]-\int_{t_{0}}^{v} D G(x(\tau), t)\right\|<\varepsilon .
$$

Portanto, temos

$$
\begin{gathered}
|x(v)(\vartheta)-x(v)(v)|<2 \varepsilon+ \\
+\left|\sum_{i}\left[G\left(x\left(\tau_{i}\right), s_{i}\right)-G\left(x\left(\tau_{i}\right), s_{i-1}\right)\right](\vartheta)-\sum_{i}\left[G\left(x\left(\tau_{i}\right), s_{i}\right)-G\left(x\left(\tau_{i}\right), s_{i-1}\right)\right](v)\right| .
\end{gathered}
$$

Pela definição de $G$ dada em (3.15), a forma de $F$ dada em (3.1) e de $J$ dada em (3.8), é fácil checar que, para todo $i$, temos

$$
G\left(x\left(\tau_{i}\right), s_{i}\right)(\vartheta)-G\left(x\left(\tau_{i}\right), s_{i-1}\right)(\vartheta)=G\left(x\left(\tau_{i}\right), s_{i}\right)(v)-G\left(x\left(\tau_{i}\right), s_{i-1}\right)(v)
$$


e isto implica, pela última desigualdade acima, que

$$
|x(v)(\vartheta)-x(v)(v)|<2 \varepsilon
$$

Com isto, para um $\varepsilon>0$ arbitrário, a relação (3.19) está satisfeita.

Para a segunda relação, suponhamos que $\vartheta \leq v$. Pela definição de uma solução de (3.18), temos analogamente como na primeira parte da demonstração

$$
x(v)(\vartheta)=x\left(t_{0}\right)(\vartheta)+\int_{t_{0}}^{v} D G(x(\tau), t)(\vartheta)
$$

$\mathrm{e}$

$$
x(\vartheta)(\vartheta)=x\left(t_{0}\right)(\vartheta)+\int_{t_{0}}^{\vartheta} D G(x(\tau), t)(\vartheta) .
$$

Portanto,

$$
x(v)(\vartheta)-x(\vartheta)(\vartheta)=\int_{\vartheta}^{v} D G(x(\tau), t)(\vartheta) .
$$

Agora, se $\left(\tau_{i},\left[s_{i-1}, s_{i}\right]\right)$ for uma divisão marcada arbitrária de $[\vartheta, v]$, novamente, é simples checar por (3.1) e (3.8) que, para todo $i$, temos

$$
G\left(x\left(\tau_{i}\right), s_{i}\right)(\vartheta)-G\left(x\left(\tau_{i}\right), s_{i-1}\right)(\vartheta)=0 .
$$

Mas isto significa que $\int_{\vartheta}^{v} D G(x(\tau), t)(\vartheta)=0$ e que vale $x(v)(\vartheta)=x(\vartheta)(\vartheta)$. Portanto (3.20) está provado.

O próximo teorema nos dará a primeira parte da relação biunívoca que será estabelecida entre a solução da EDFRI (2.13) e a solução da EDOG (3.18).

Teorema 3.2 ([7], Teorema 3.4). Consideremos a equação (2.13), onde para todo $y \in$ $P C_{1}, f: G^{-}\left([-r, 0], \mathbb{R}^{n}\right) \times\left[t_{0}, t_{0}+\sigma\right] \rightarrow \mathbb{R}^{n}$ é tal que $t \mapsto f\left(y_{t}, t\right)$ é Lebesgue integrável em $\left[t_{0}, t_{0}+\sigma\right]$ e (A), (B), (A'), (B') estão satisfeitas. Seja y $(t)$ uma solução do problema (2.13) no intervalo $\left[t_{0}, t_{0}+\sigma\right]$. Dado $t \in\left[t_{0}-r, t_{0}+\sigma\right]$, seja

$$
x(t)(\vartheta)=\left\{\begin{array}{l}
y(\vartheta), \vartheta \in\left[t_{0}-r, t\right] \\
y(t), \vartheta \in\left[t, t_{0}+\sigma\right] .
\end{array}\right.
$$

Então $x(t) \in G^{-}\left(\left[t_{0}-r, t_{0}+\sigma\right], \mathbb{R}^{n}\right)$ será uma solução de $(3.18)$ em $\left[t_{0}-r, t_{0}+\sigma\right]$.

Prova: Precisamos mostrar que, para todo $v \in\left[t_{0}, t_{0}+\sigma\right]$, a integral $\int_{t_{0}}^{v} D G(x(\tau), t)$ existe e vale

$$
x(v)-x\left(t_{0}\right)=\int_{t_{0}}^{v} D G(x(\tau), t) .
$$


Consideremos um $\varepsilon>0$ arbitrário dado.

Como y é uma solução de (2.13), as relações (1.1) referentes à forma "integral" equivalente estão satisfeitas e a função $y:\left[t_{0}, t_{0}+\sigma\right] \rightarrow \mathbb{R}^{n}$ pode ser escrita é a soma de uma função absolutamente contínua e uma função escada finita contínua à esquerda.

Desta forma, para todo $\tau \in\left[t_{0}, t_{0}+\sigma\right]$, existe $u m \delta(\tau)>0$ tal que

$$
|y(\rho)-y(\tau)|<\varepsilon \text { para todo } \rho \in[\tau-\delta(\tau), \tau]
$$

e

$$
|y(\rho)-y(\tau+)|<\varepsilon \text { para todo } \rho \in(\tau, \tau+\delta(\tau)] .
$$

Portanto, temos naturalmente um calibre $\delta$ de $\left[t_{0}, t_{0}+\sigma\right]$. E mais, consideremos o calibre $\delta$ de modo que, se $\tau \in\left[t_{0}, t_{0}+\sigma\right]$, então

$$
\left|\int_{u}^{v} L(s) d s\right|<\frac{\varepsilon}{(m+1)\left(K_{1}+1\right)}, \text { para todo }[u, v] \subset(\tau-\delta(\tau), \tau+\delta(\tau))
$$

onde $m$ é o número de momentos de impulsos no intervalo $\left[t_{0}, t_{0}+\sigma\right]$ e $K_{1}$ é a constante da condição (A'). Esta tal escolha é possível pois a função $L:\left[t_{0}, t_{0}+\sigma\right] \rightarrow \mathbb{R}$ da condição (B) é Lebesgue integrável. Suponhamos, ainda, que o calibre $\delta$ satisfaça as seguintes condições

$$
0<\delta(\tau)<\min \left\{\frac{t_{k}-t_{k-1}}{2} ; k=1, \ldots, m\right\}
$$

$\mathrm{e}$

$$
0<\delta(\tau)<\min \left\{\operatorname{dist}\left(\tau, t_{k}\right), \operatorname{dist}\left(\tau, t_{k-1}\right) ; \tau \in\left(t_{k-1}, t_{k}\right), k=1, \ldots, m\right\},
$$

onde $\operatorname{dist}\left(\tau, t_{k}\right)$ denota a distância de $\tau$ a $t_{k}$ e similarmente para $\operatorname{dist}\left(\tau, t_{k-1}\right)$.

A condição (3.25) assegura que se um intervalo marcado $\left(T,\left[s_{1}, s_{2}\right]\right)$ for $\delta$-fino, então o intervalo $\left[s_{1}, s_{2}\right]$ conterá no máximo um ponto $t_{k}, k=1, \ldots, m$, enquanto (3.26) implica que $T=t_{k}$ sempre que $t_{k} \in\left[s_{1}, s_{2}\right]$.

Suponhamos que $\left(\tau_{i},\left[s_{i-1}, s_{i}\right]\right)$ seja uma partição $\delta$-fina do intervalo $\left[t_{0}, v\right]$. Usando a definição (3.21) de $x$ e (1.1), podemos mostrar facilmente que

$$
\begin{aligned}
& {\left[x\left(s_{i}\right)-x\left(s_{i-1}\right)\right](\vartheta)=} \\
& =\left\{\begin{array}{l}
0, \quad \vartheta \in\left[t_{0}-r, s_{i-1}\right] \\
\int_{s_{i-1}}^{\vartheta} f\left(y_{s}, s\right) d s+\sum_{k=0}^{m} I_{k}\left(y\left(t_{k}\right)\right)\left[H_{t_{k}}(\vartheta)-H_{t_{k}}\left(s_{i-1}\right)\right], \vartheta \in\left[s_{i-1}, s_{i}\right], \\
\int_{s_{i-1}}^{s_{i}} f\left(y_{s}, s\right) d s+\sum_{k=0}^{m} I_{k}\left(y\left(t_{k}\right)\right)\left[H_{t_{k}}\left(s_{i}\right)-H_{t_{k}}\left(s_{i-1}\right)\right], \vartheta \in\left[s_{i}, t_{0}+\sigma\right] .
\end{array}\right.
\end{aligned}
$$


Usando a definição de $G$ dada em (3.15) e usando (3.1) e (3.8) obtemos

$$
\begin{gathered}
{\left[G\left(x\left(\tau_{i}\right), s_{i}\right)-G\left(x\left(\tau_{i}\right), s_{i-1}\right)\right](\vartheta)=} \\
=\left[F\left(x\left(\tau_{i}\right), s_{i}\right)-F\left(x\left(\tau_{i}\right), s_{i-1}\right)\right](\vartheta)+\sum_{k=0}^{m}\left[H_{t_{k}}\left(s_{i}\right)-H_{t_{k}}\left(s_{i-1}\right)\right] H_{t_{k}}(\vartheta) I_{k}\left(x\left(\tau_{i}\right)\left(t_{k}\right)\right)= \\
=\left\{\begin{array}{l}
0, \vartheta \in\left[t_{0}-r, s_{i-1}\right] \\
\int_{s_{i-1}}^{\vartheta} f\left(x\left(\tau_{i}\right)_{s}, s\right) d s, \vartheta \in\left[s_{i-1}, s_{i}\right] \\
\int_{s_{i-1}}^{s_{i}} f\left(x\left(\tau_{i}\right)_{s}, s\right) d s, \vartheta \in\left[s_{i}, t_{0}+\sigma\right]
\end{array}\right\}+ \\
+\sum_{k=0}^{m}\left[H_{t_{k}}\left(s_{i}\right)-H_{t_{k}}\left(s_{i-1}\right)\right] H_{t_{k}}(\vartheta) I_{k}\left(x\left(\tau_{i}\right)\left(t_{k}\right)\right) .
\end{gathered}
$$

Usando as propriedades (3.25) e (3.26) do calibre $\delta$ e as propriedades correspondentes da partição $\left(\tau_{i},\left[s_{i-1}, s_{i}\right]\right)$, existem duas possibilidades para um dado intervalo marcado $\left(\tau_{i},\left[s_{i-1}, s_{i}\right]\right)$ :

(i) existe exatamente um $t_{l} \in\left[s_{i-1}, s_{i}\right)$,

(ii) $\left[s_{i-1}, s_{i}\right)$ não contém nenhum ponto de impulso, isto é, $\left[s_{i-1}, s_{i}\right) \cap\left\{t_{0}, \ldots, t_{m}\right\}=\emptyset$. No caso (i), temos

$$
\sum_{k=0}^{m} I_{k}\left(y\left(t_{k}\right)\right)\left[H_{t_{k}}(\vartheta)-H_{t_{k}}\left(s_{i-1}\right)\right]=I_{l}\left(y\left(t_{l}\right)\right) H_{t_{l}}(\vartheta)
$$

e, como $\tau_{i}=t_{l}$, obtemos, pela definição de $x$,

$$
\sum_{k=0}^{m}\left[H_{t_{k}}\left(s_{i}\right)-H_{t_{k}}\left(s_{i-1}\right)\right] H_{t_{k}}(\vartheta) I_{k}\left(x\left(\tau_{i}\right)\left(t_{k}\right)\right)=I_{l}\left(x\left(\tau_{i}\right)\left(t_{l}\right)\right) H_{t_{l}}(\vartheta)=I_{l}\left(y\left(t_{l}\right)\right) H_{t_{l}}(\vartheta) .
$$

Daí, por (3.27) e (3.28), temos

$$
\begin{gathered}
{\left[x\left(s_{i}\right)-x\left(s_{i-1}\right)\right](\vartheta)-\left[G\left(x\left(\tau_{i}\right), s_{i}\right)-G\left(x\left(\tau_{i}\right), s_{i-1}\right)\right](\vartheta)=} \\
=\left\{\begin{array}{l}
0, \vartheta \in\left[t_{0}-r, s_{i-1}\right] \\
\int_{s_{i-1}}^{\vartheta} f\left(y_{s}, s\right) d s-\int_{s_{i-1}}^{\vartheta} f\left(x\left(\tau_{i}\right)_{s}, s\right) d s, \vartheta \in\left[s_{i-1}, s_{i}\right],= \\
\int_{s_{i-1}}^{s_{i}} f\left(y_{s}, s\right) d s-\int_{s_{i-1}}^{s_{i}} f\left(x\left(\tau_{i}\right)_{s}, s\right) d s, \vartheta \in\left[s_{i}, t_{0}+\sigma\right]
\end{array}\right.
\end{gathered}
$$




$$
=\left\{\begin{array}{l}
0, \vartheta \in\left[t_{0}-r, s_{i-1}\right] \\
\int_{s_{i-1}}^{\vartheta}\left[f\left(y_{s}, s\right)-f\left(x\left(\tau_{i}\right)_{s}, s\right)\right] d s, \vartheta \in\left[s_{i-1}, s_{i}\right], \\
\int_{s_{i-1}}^{s_{i}}\left[f\left(y_{s}, s\right)-f\left(x\left(\tau_{i}\right)_{s}, s\right)\right] d s, \vartheta \in\left[s_{i}, t_{0}+\sigma\right] .
\end{array}\right.
$$

No caso (ii), temos

$$
\sum_{k=0}^{m} I_{k}\left(y\left(t_{k}\right)\right)\left[H_{t_{k}}(\vartheta)-H_{t_{k}}\left(s_{i-1}\right)\right]=0
$$

e também

$$
\sum_{k=0}^{m}\left[H_{t_{k}}\left(s_{i}\right)-H_{t_{k}}\left(s_{i-1}\right)\right] H_{t_{k}}(\vartheta) I_{k}\left(x\left(\tau_{i}\right)\left(t_{k}\right)\right)=0 .
$$

Logo, por (3.27) e (3.28), obtemos a relação (3.29) novamente.

Usando (3.29), também temos

$$
\begin{gathered}
\left\|x\left(s_{i}\right)-x\left(s_{i-1}\right)-\left[G\left(x\left(\tau_{i}\right), s_{i}\right)-G\left(x\left(\tau_{i}\right), s_{i-1}\right)\right]\right\|= \\
=\sup _{\vartheta \in\left[t_{0}-r, t_{0}+\sigma\right]}\left|\left[x\left(s_{i}\right)-x\left(s_{i-1}\right)\right](\vartheta)-\left[G\left(x\left(\tau_{i}\right), s_{i}\right)-G\left(x\left(\tau_{i}\right), s_{i-1}\right)\right](\vartheta)\right|= \\
=\sup _{\vartheta \in\left[t_{0}-r, t_{0}+\sigma\right]}\left\{\begin{array}{l}
\left|\int_{s_{i-1}}^{\vartheta}\left[f\left(y_{s}, s\right)-f\left(x\left(\tau_{i}\right)_{s}, s\right)\right] d s\right|, \vartheta \in\left[s_{i-1}, s_{i}\right] \\
\left|\int_{s_{i-1}}^{s_{i}}\left[f\left(y_{s}, s\right)-f\left(x\left(\tau_{i}\right)_{s}, s\right)\right] d s\right|, \vartheta \in\left[s_{i}, t_{0}+\sigma\right] \\
=\sup _{\vartheta \in\left[s_{i-1}, s_{i}\right]}\left|\int_{s_{i-1}}^{\vartheta}\left[f\left(y_{s}, s\right)-f\left(x\left(\tau_{i}\right)_{s}, s\right)\right] d s\right| .
\end{array}\right.
\end{gathered}
$$

Pela definição de $x$ proveniente de (3.21), temos para o caso (i),

$$
\int_{s_{i-1}}^{\vartheta}\left[f\left(y_{s}, s\right)-f\left(x\left(\tau_{i}\right)_{s}, s\right)\right] d s=\int_{t_{l}}^{\vartheta}\left[f\left(y_{s}, s\right)-f\left(x\left(t_{l}\right)_{s}, s\right)\right] d s,
$$

para $\vartheta \in\left[t_{l}, s_{i}\right], \mathrm{e}$

$$
\int_{s_{i-1}}^{\vartheta}\left[f\left(y_{s}, s\right)-f\left(x\left(\tau_{i}\right)_{s}, s\right)\right] d s=0
$$

para $\vartheta \in\left[s_{i-1}, t_{l}\right]$. Pela condição $(\mathrm{B})$, temos

$$
\left|\int_{t_{l}}^{\vartheta}\left[f\left(y_{s}, s\right)-f\left(x\left(t_{l}\right)_{s}, s\right)\right] d s\right| \leq \int_{t_{l}}^{\vartheta} L(s)\left\|y_{s}-x\left(t_{l}\right)_{s}\right\| d s, \quad \vartheta \in\left[t_{l}, s_{i}\right] .
$$


Usando (3.22) e (B'), então para $s \in\left[t_{l}, s_{i}\right]$, temos

$$
\begin{gathered}
\left\|y_{s}-x\left(t_{l}\right)_{s}\right\|=\sup _{\rho \in[-r, 0]}\left|y(s+\rho)-x\left(t_{l}\right)(s+\rho)\right|= \\
=\sup _{\rho \in\left[t_{l}, s\right]}\left|y(\rho)-y\left(t_{l}\right)\right|=\sup _{\rho \in\left[t_{l}, s\right]}\left|y(\rho)-y\left(t_{l}+\right)+y\left(t_{l}+\right)-y\left(t_{l}\right)\right|= \\
=\sup _{\rho \in\left[t_{l}, s\right]}\left|y(\rho)-y\left(t_{l}+\right)+I_{l} y\left(t_{l}\right)\right| \leq \varepsilon+K_{1} .
\end{gathered}
$$

Portanto, pela propriedade (3.24) do calibre $\delta$, temos

$$
\begin{gathered}
\left\|x\left(s_{i}\right)-x\left(s_{i-1}\right)-\left[G\left(x\left(\tau_{i}\right), s_{i}\right)-G\left(x\left(\tau_{i}\right), s_{i-1}\right)\right]\right\| \leq\left(\varepsilon+K_{1}\right) \int_{t_{l}}^{s_{i}} L(s) d s \leq \\
\quad \leq \varepsilon \int_{t_{l}}^{s_{i}} L(s) d s+K_{1} \frac{\varepsilon}{(m+1)\left(K_{1}+1\right)}<\varepsilon \int_{t_{l}}^{s_{i}} L(s) d s+\frac{\varepsilon}{(m+1)} .
\end{gathered}
$$

Similarmente para o caso (ii), temos

$$
\int_{s_{i-1}}^{\vartheta}\left[f\left(y_{s}, s\right)-f\left(x\left(\tau_{i}\right)_{s}, s\right)\right] d s=\int_{\tau_{i}}^{\vartheta}\left[f\left(y_{s}, s\right)-f\left(x\left(\tau_{i}\right)_{s}, s\right)\right] d s
$$

$\operatorname{para} \vartheta \in\left[\tau_{i}, s_{i}\right] \mathrm{e}$

$$
\int_{s_{i-1}}^{\vartheta}\left[f\left(y_{s}, s\right)-f\left(x\left(\tau_{i}\right)_{s}, s\right)\right] d s=0
$$

para $\vartheta \in\left[s_{i-1}, \tau_{i}\right]$.

Pela condição $(\mathrm{B})$, temos

$$
\left|\int_{\tau_{i}}^{\vartheta}\left[f\left(y_{s}, s\right)-f\left(x\left(\tau_{i}\right)_{s}, s\right)\right] d s\right| \leq \int_{\tau_{i}}^{\vartheta} L(s)\left\|y_{s}-x\left(\tau_{i}\right)_{s}\right\| d s, \vartheta \in\left[\tau_{i}, s_{i}\right] .
$$

onde

$$
\left\|y_{s}-x\left(\tau_{i}\right)_{s}\right\|=\sup _{\rho \in\left[\tau_{i}, s\right]}\left|y(\rho)-y\left(\tau_{i}\right)\right| \leq \varepsilon, s \in\left[\tau_{i}, s_{i}\right],
$$

pela propriedade do calibre $\delta$ dada em (3.23) .

Logo,

$$
\left\|x\left(s_{i}\right)-x\left(s_{i-1}\right)-\left[G\left(x\left(\tau_{i}\right), s_{i}\right)-G\left(x\left(\tau_{i}\right), s_{i-1}\right)\right]\right\| \leq \varepsilon \int_{\tau_{i}}^{s_{i}} L(s) d s .
$$

Usando os resultados obtidos acima e o fato de que o caso (i) ocorre em no máximo 
$m$ intervalos, temos

$$
\begin{aligned}
& \left\|x(v)-x\left(t_{0}\right)-\sum_{i}\left[G\left(x\left(\tau_{i}\right), s_{i}\right)-G\left(x\left(\tau_{i}\right), s_{i-1}\right)\right]\right\|= \\
= & \left\|\sum_{i}\left\{x\left(s_{i}\right)-x\left(s_{i-1}\right)-\left[G\left(x\left(\tau_{i}\right), s_{i}\right)-G\left(x\left(\tau_{i}\right), s_{i-1}\right)\right]\right\}\right\| \leq \\
\leq & \sum_{i}\left\|x\left(s_{i}\right)-x\left(s_{i-1}\right)-\left[G\left(x\left(\tau_{i}\right), s_{i}\right)-G\left(x\left(\tau_{i}\right), s_{i-1}\right)\right]\right\| \leq \\
\leq & \sum_{i ; t_{l} \in\left[s_{i-1}, s_{i}\right)} \varepsilon \int_{t_{l}}^{s_{i}} L(s) d s+\frac{\varepsilon}{(m+1)}+\sum_{i} \varepsilon \int_{\tau_{i}}^{s_{i}} L(s) d s< \\
& <2 \varepsilon \int_{t_{0}}^{t_{0}+\sigma} L(s) d s+m \frac{\varepsilon}{(m+1)}<2 \varepsilon \int_{t_{0}}^{t_{0}+\sigma} L(s) d s+\varepsilon
\end{aligned}
$$

Portanto, para todo $v \in\left[t_{0}, t_{0}+\sigma\right]$ a integral $\int_{t_{0}}^{v} D G(x(\tau), t)$ existe e

$$
x(v)-x\left(t_{0}\right)=\int_{t_{0}}^{v} D G(x(\tau), t)
$$

Isto prova o resultado.

Teorema 3.3 ([7], Teorema 3.5). Seja $G$ dada por (3.15) e seja $x(t)$ uma solução de (3.18), no intervalo $\left[t_{0}-r, t_{0}+\sigma\right]$ satisfazendo a condição inicial

$$
x\left(t_{0}\right)(\vartheta)=\left\{\begin{array}{l}
\phi\left(\vartheta-t_{0}\right), t_{0}-r \leq \vartheta \leq t_{0}, \\
x\left(t_{0}\right)\left(t_{0}\right), t_{0} \leq \vartheta \leq t_{0}+\sigma
\end{array}\right.
$$

Para todo $\vartheta \in\left[t_{0}-r, t_{0}+\sigma\right]$, consideremos

$$
y(\vartheta)=\left\{\begin{array}{l}
x\left(t_{0}\right)(\vartheta), t_{0}-r \leq \vartheta \leq t_{0} \\
x(\vartheta)(\vartheta), \quad t_{0} \leq \vartheta \leq t_{0}+\sigma .
\end{array}\right.
$$

Então y $(\vartheta)$ é uma solução do problema (2.13) em $\left[t_{0}-r, t_{0}+\sigma\right]$ e $y(\vartheta)=x\left(t_{0}+\sigma\right)(\vartheta)$, $\vartheta \in\left[t_{0}-r, t_{0}+\sigma\right]$.

Prova: De acordo com (2.14), é suficiente provar que, para todo $\eta>0$ e para algum $v \in\left[t_{0}, t_{0}+\sigma\right]$, temos

$$
\left|y(v)-y\left(t_{0}\right)-\int_{t_{0}}^{v} f\left(y_{s}, s\right) d s-\sum_{k=1}^{m} I_{k}\left(y\left(t_{k}\right)\right) H_{t_{k}}(v)\right|<\eta
$$


e

$$
y_{t_{0}}=\phi
$$

A última igualdade está clara por (3.30).

Suponhamos que um calibre $\delta:\left[t_{0}, t_{0}+\sigma\right] \rightarrow(0,+\infty)$ satisfaça, para $\tau \in\left[t_{0}, t_{0}+\sigma\right]$, as seguintes desigualdades:

$$
0<\delta(\tau)<\min \left\{\frac{t_{k}-t_{k-1}}{2} ; k=1, \ldots, m\right\}
$$

e

$$
0<\delta(\tau)<\min \left\{\operatorname{dist}\left(\tau, t_{k}\right), \operatorname{dist}\left(\tau, t_{k-1}\right) \text { para } \tau \in\left(t_{k-1}, t_{k}\right), k=1, \ldots, m\right\}
$$

onde $\operatorname{dist}\left(\tau, t_{k}\right)$ é a distância de $\tau$ a $t_{k}$ e similarmente para $\operatorname{dist}\left(\tau, t_{k-1}\right)$.

Como na prova do Teorema 3.2, a condição (3.32) assegura que se um intervalo marcado $\left(T,\left[s_{1}, s_{2}\right]\right)$ for $\delta$-fino, então o intervalo $\left[s_{1}, s_{2}\right]$ conterá no máximo um dos pontos $t_{k}, k=1, \ldots, m$, enquanto (3.33) implica que $T=t_{k}$ para $t_{k} \in\left[s_{1}, s_{2}\right]$.

Se $\left(\tau_{i},\left[s_{i-1}, s_{i}\right]\right)$ for uma partição $\delta$-fina de $\left[t_{0}, v\right]$, então por $(3.11)$, quando $t_{l} \in$ $\left[s_{i-1}, s_{i}\right)$, teremos

$$
\begin{gathered}
J\left(x\left(\tau_{i}\right), s_{i}\right)(\vartheta)-J\left(x\left(\tau_{i}\right), s_{i-1}\right)(\vartheta)=\sum_{k=1}^{m}\left[H_{t_{k}}\left(s_{2}\right)-H_{t_{k}}\left(s_{1}\right)\right] H_{t_{k}}(\vartheta) I_{k}\left(x\left(\tau_{i}\right)\left(t_{k}\right)\right)= \\
=H_{t_{l}}(\vartheta) I_{k}\left(x\left(t_{l}\right)\left(t_{l}\right)\right)=H_{t_{l}}(\vartheta) I_{k}\left(y\left(t_{l}\right)\right)
\end{gathered}
$$

para $\vartheta \in\left[t_{0}-r, t_{0}+\sigma\right]$, e se $\left[s_{i-1}, s_{i}\right)$ não contiver nenhum ponto $t_{1}, \ldots, t_{m}$, então

$$
J\left(x\left(\tau_{i}\right), s_{i}\right)(\vartheta)-J\left(x\left(\tau_{i}\right), s_{i-1}\right)(\vartheta)=\sum_{k=1}^{m}\left[H_{t_{k}}\left(s_{2}\right)-H_{t_{k}}\left(s_{1}\right)\right] H_{t_{k}}(\vartheta) I_{k}\left(x\left(\tau_{i}\right)\left(t_{k}\right)\right)=0 .
$$

Isto implica que a integral $\int_{t_{0}}^{v} D J(x(\tau), t)$ existe e

$$
\left[\int_{t_{0}}^{v} D J(x(\tau), t)\right](v)=\sum_{k=1}^{m} H_{t_{k}}(v) I_{k}\left(y\left(t_{k}\right)\right)
$$

Por (3.30), (3.19) e pelo fato de que $x$ é uma solução de (3.18) temos

$$
y(v)-y\left(t_{0}\right)=x(v)(v)-x\left(t_{0}\right)\left(t_{0}\right)=x(v)(v)-x\left(t_{0}\right)(v)=
$$




$$
=\left[\int_{t_{0}}^{v} D G(x(\tau), t)\right](v)=\left[\int_{t_{0}}^{v} D F(x(\tau), t)\right](v)+\left[\int_{t_{0}}^{v} D J(x(\tau), t)\right](v) .
$$

para $v \in\left[t_{0}, t_{0}+\sigma\right]$.

Usando (3.35) e (3.34), obtemos

$$
\begin{gathered}
y(v)-y\left(t_{0}\right)-\int_{t_{0}}^{v} f\left(y_{s}, s\right) d s-\sum_{k=1}^{m} I_{k}\left(y\left(t_{k}\right)\right) H_{t_{k}}(v)= \\
=\left[\int_{t_{0}}^{v} D F(x(\tau), t)\right](v)-\int_{t_{0}}^{v} f\left(y_{s}, s\right) d s .
\end{gathered}
$$

Note que a existência das integrais $\int_{t_{0}}^{v} D G(x(\tau), t)$ e $\int_{t_{0}}^{v} D J(x(\tau), t)$ implicam a existência da integral $\int_{t_{0}}^{v} D F(x(\tau), t)$.

Seja $\varepsilon>0$ dado.

Suponhamos que um calibre $\delta(\tau)>0$ de $\left[t_{0}, t_{0}+\sigma\right]$ satisfaça (3.32), (3.33) e as desigualdades

$$
|h(\rho)-h(\tau)|<\varepsilon, \text { para todo } \rho \in[\tau-\delta(\tau), \tau]
$$

e

$$
|h(\rho)-h(\tau+)|<\varepsilon, \text { para todo } \rho \in(\tau, \tau+\delta(\tau)] .
$$

onde $h(t)=h_{1}(t)+h_{2}(t)$ é a função não decrescente e contínua à esquerda descrita por (3.16) e (3.17).

Consideremos, também, o calibre $\delta$ de modo que se $\tau \in\left[t_{0}, t_{0}+\sigma\right]$, então

$$
\left|\int_{u}^{v} L(s) d s\right|<\frac{\varepsilon}{(m+1)\left(K_{1}+1\right)}, \text { para todo }[u, v] \subset[\tau-\delta(\tau), \tau+\delta(\tau)]
$$

onde $m$ é o número de pontos de impulso e $K_{1}$ é a constante da condição (A'). Tal escolha é possível, pois a função $L:\left[t_{0}, t_{0}+\sigma\right] \rightarrow \mathbb{R}$ da condição (B) é Lebesgue integrável.

Para o calibre $\delta$ que acabamos de definir, temos, pela existência da integral $\int_{t_{0}}^{v} D F(x(\tau), t)$, a desigualdade

$$
\left\|\int_{t_{0}}^{v} D F(x(\tau), t)-\sum_{i}\left[F\left(x\left(\tau_{i}\right), s_{i}\right)-F\left(x\left(\tau_{i}\right), s_{i-1}\right)\right]\right\|<\varepsilon
$$

para toda partição $\delta$-fina $\left(\tau_{i},\left[s_{i-1}, s_{i}\right]\right)$ do intervalo $\left[t_{0}, v\right]$. Portanto

$$
\left|\int_{t_{0}}^{v} D F(x(\tau), t)(v)-\sum_{i}\left[F\left(x\left(\tau_{i}\right), s_{i}\right)-F\left(x\left(\tau_{i}\right), s_{i-1}\right)\right](v)\right|<\varepsilon
$$


para toda partição $\delta$-fina $\left(\tau_{i},\left[s_{i-1}, s_{i}\right]\right)$ do intervalo $\left[t_{0}, v\right]$. Por $(3.36)$ e $(3.41)$, temos

$$
\begin{gathered}
\left|y(v)-y\left(t_{0}\right)-\int_{t_{0}}^{v} f\left(y_{s}, s\right) d s-\sum_{k=1}^{m} I_{k}\left(y\left(t_{k}\right)\right) H_{t_{k}}(v)\right|= \\
=\left|\left(\int_{t_{0}}^{v} D F(x(\tau), t)\right)(v)-\int_{t_{0}}^{v} f\left(y_{s}, s\right) d s\right|< \\
\left.<\varepsilon+\mid \sum_{i}\left[F\left(x\left(\tau_{i}\right), s_{i}\right)-F\left(x\left(\tau_{i}\right), s_{i-1}\right)\right]\right](v)-\int_{t_{0}}^{v} f\left(y_{s}, s\right) d s \mid= \\
=\varepsilon+\left|\sum_{i}\left\{\left[F\left(x\left(\tau_{i}\right), s_{i}\right)-F\left(x\left(\tau_{i}\right), s_{i-1}\right)\right](v)-\int_{s_{i-1}}^{s_{i}} f\left(y_{s}, s\right) d s\right\}\right|
\end{gathered}
$$

A definição de $F$ dada em (3.1) implica

$$
\left[F\left(x\left(\tau_{i}\right), s_{i}\right)-F\left(x\left(\tau_{i}\right), s_{i-1}\right)\right](v)=\int_{s_{i-1}}^{s_{i}} f\left(x\left(\tau_{i}\right)_{s}, s\right) d s
$$

Por $(3.20)$, temos $x\left(\tau_{i}\right)(\vartheta)=x(\vartheta)(\vartheta)=y(\vartheta)$, para $\vartheta \leq \tau_{i}$. Portanto

$$
\begin{aligned}
\int_{s_{i-1}}^{s_{i}} f\left(x\left(\tau_{i}\right)_{s}, s\right) d s & -\int_{s_{i-1}}^{s_{i}} f\left(y_{s}, s\right) d s=\int_{s_{i-1}}^{s_{i}}\left[f\left(x\left(\tau_{i}\right)_{s}, s\right)-f\left(y_{s}, s\right)\right] d s= \\
& =\int_{\tau_{i}}^{s_{i}}\left[f\left(x\left(\tau_{i}\right)_{s}, s\right)-f\left(y_{s}, s\right)\right] d s .
\end{aligned}
$$

Analogamente, para $\vartheta \in\left[\tau_{i}, s_{i}\right]$, temos, novamente por $(3.20)$, a igualdade $y(\vartheta)=x(\vartheta)(\vartheta)=$ $x\left(s_{i}\right)(\vartheta)$ e portanto

$$
\int_{\tau_{i}}^{s_{i}}\left[f\left(x\left(\tau_{i}\right)_{s}, s\right)-f\left(y_{s}, s\right)\right] d s=\int_{\tau_{i}}^{s_{i}}\left[f\left(x\left(\tau_{i}\right)_{s}, s\right)-f\left(x\left(s_{i}\right)_{s}, s\right)\right] d s .
$$

Usando as relações acima e a condição (B), obtemos

$$
\begin{gathered}
\left|\left[F\left(x\left(\tau_{i}\right), s_{i}\right)-F\left(x\left(\tau_{i}\right), s_{i-1}\right)\right](v)-\int_{s_{i-1}}^{s_{i}} f\left(y_{s}, s\right) d s\right|= \\
=\left|\int_{\tau_{i}}^{s_{i}}\left[f\left(x\left(\tau_{i}\right)_{s}, s\right)-f\left(x\left(s_{i}\right)_{s}, s\right)\right] d s\right| \leq \int_{\tau_{i}}^{s_{i}} L(s)\left\|x\left(\tau_{i}\right)_{s}-x\left(s_{i}\right)_{s}\right\| d s .
\end{gathered}
$$

Agora, vamos analisar $\left\|x\left(\tau_{i}\right)_{s}-x\left(s_{i}\right)_{s}\right\|$. Pela definição e pelo fato de que $x\left(s_{i}\right)\left(\tau_{i}\right)=$ 
$x\left(\tau_{i}\right)\left(\tau_{i}\right)$ (veja $\left.(3.20)\right)$, temos para todo $i$ a seguinte relação:

$$
\begin{gathered}
\left\|x\left(\tau_{i}\right)_{s}-x\left(s_{i}\right)_{s}\right\|=\sup _{\vartheta \in[-r, 0]}\left|x\left(s_{i}\right)(s+\vartheta)-x\left(\tau_{i}\right)(s+\vartheta)\right|= \\
=\sup _{\rho \in\left[\tau_{i}, s_{i}\right]}\left|x\left(s_{i}\right)(\rho)-x\left(\tau_{i}\right)(\rho)\right|=\sup _{\rho \in\left(\tau_{i}, s_{i}\right]}\left|x\left(s_{i}\right)(\rho)-x\left(\tau_{i}\right)(\rho)\right|= \\
=\sup _{\rho \in\left(\tau_{i}, s_{i}\right]}\left|x\left(s_{i}\right)(\rho)-x\left(\tau_{i}+\right)(\rho)+x\left(\tau_{i}+\right)(\rho)-x\left(\tau_{i}\right)(\rho)\right| \leq \\
\leq \sup _{\rho \in\left(\tau_{i}, s_{i}\right]}\left\{\left|x\left(s_{i}\right)(\rho)-x\left(\tau_{i}+\right)(\rho)\right|+\left|x\left(\tau_{i}+\right)(\rho)-x\left(\tau_{i}\right)(\rho)\right|\right\} \leq \\
\leq\left\|x\left(s_{i}\right)-x\left(\tau_{i}+\right)\right\|+\left\|G\left(x\left(\tau_{i}\right), \tau_{i}+\right)-G\left(x\left(\tau_{i}\right), \tau_{i}\right)\right\| \leq h\left(s_{i}\right)-h\left(\tau_{i}+\right)+K_{1}<\varepsilon+K_{1},
\end{gathered}
$$

onde as últimas desigualdades vêm do Lema 1.22 e da definição da $G$ em (3.15). Logo por (3.43), para todo $i$, vale a desigualdade

$$
\begin{aligned}
& \left|\left[F\left(x\left(\tau_{i}\right), s_{i}\right)-F\left(x\left(\tau_{i}\right), s_{i-1}\right)\right](v)-\int_{s_{i-1}}^{s_{i}} f\left(y_{s}, s\right) d s\right| \leq \\
& \leq \int_{\tau_{i}}^{s_{i}} L(s)\left\|x\left(\tau_{i}\right)_{s}-x\left(s_{i}\right)_{s}\right\| d s<\left(\varepsilon+K_{1}\right) \int_{\tau_{i}}^{s_{i}} L(s) d s .
\end{aligned}
$$

Agora, por (3.42) e (3.44), obtemos

$$
\begin{gathered}
\left|y(v)-y\left(t_{0}\right)-\int_{t_{0}}^{v} f\left(y_{s}, s\right) d s-\sum_{k=1}^{m} I_{k}\left(y\left(t_{k}\right)\right) H_{t_{k}}(v)\right|< \\
<\varepsilon+\sum_{i}\left|\left[F\left(x\left(\tau_{i}\right), s_{i}\right)-F\left(x\left(\tau_{i}\right), s_{i-1}\right)\right](v)-\int_{s_{i-1}}^{s_{i}} f\left(y_{s}, s\right) d s\right|< \\
<\varepsilon+\left(\varepsilon+K_{1}\right) \sum_{i} \int_{\tau_{i}}^{s_{i}} L(s) d s \leq \\
\leq \varepsilon+\varepsilon \int_{t_{0}}^{v} L(s) d s+K_{1} \sum_{i ;\left[s_{i-1}, s_{i}\right) \cap\left\{t_{1}, \ldots, t_{m}\right\} \neq \emptyset} \int_{\tau_{i}}^{s_{i}} L(s) d s \leq \\
\leq \varepsilon\left(1+\int_{t_{0}}^{v} L(s) d s\right)+m \cdot K_{1} \frac{\varepsilon}{(m+1)\left(K_{1}+1\right)} \leq \varepsilon\left(1+\int_{t_{0}}^{v} L(s) d s\right)+\varepsilon
\end{gathered}
$$

pela propriedade do calibre (3.39). Daí, tomando $\varepsilon>0$ tal que

$$
\varepsilon\left(2+\int_{t_{0}}^{v} L(s) d s\right)<\eta,
$$

obtemos (3.31) e o teorema está provado. 


\subsection{Dependência contínua para EDFRIs}

Consideraremos a seguinte sequência de problemas de valor inicial para EDFRIs

$$
\left\{\begin{array}{l}
\dot{y}(t)=f_{p}\left(y_{t}, t\right), t \neq t_{k} \\
\Delta y\left(t_{k}\right)=I_{k}^{p}\left(y\left(t_{k}\right)\right), k=0,1, \ldots, m \\
y_{t_{0}}=\phi_{p}
\end{array}\right.
$$

onde $p=0,1,2, \ldots$ Suponhamos que, para $p=0,1, \ldots$, temos $\phi_{p} \in G^{-}\left([-r, 0], \mathbb{R}^{n}\right)$ e as funções $f_{p}, I_{k}^{P}$ satisfazem as condições (A), (B), (A') e (B') para as mesmas funções $M$ e $L$ e as mesmas constantes $K_{1}$ e $K_{2}$ para todo $p=0,1, \ldots$ Então, para todo $p=0,1, \ldots$, o sistema (3.46) é equivalente a

$$
\left\{\begin{array}{l}
y(t)=y\left(t_{0}\right)+\int_{t_{0}}^{t} f_{p}\left(y_{s}, s\right) d s+\sum_{k=1}^{m} I_{k}^{p}\left(y\left(t_{k}\right)\right) H_{t_{k}}(t), t \in\left[t_{0}, t_{0}+\sigma\right] \\
y_{t_{0}}=\phi_{p} .
\end{array}\right.
$$

Definindo, para $p=0,1, \ldots, y \in P C_{1}$ e $t \in\left[t_{0}-r, t_{0}+\sigma\right]$, as funções

$$
F_{p}(y, t)(\vartheta)=\left\{\begin{array}{l}
0, t_{0}-r \leq \vartheta \leq t_{0} \text { or } t_{0}-r \leq t \leq t_{0} \\
\int_{t_{0}}^{\vartheta} f_{p}\left(y_{s}, s\right) d s, \quad t_{0} \leq \vartheta \leq t \leq t_{0}+\sigma \\
\int_{t_{0}}^{t} f_{p}\left(y_{s}, s\right) d s, \quad t_{0} \leq t \leq \vartheta \leq t_{0}+\sigma
\end{array}\right.
$$

$\mathrm{e}$

$$
J_{p}(y, t)(\vartheta)=\sum_{k=1}^{m} H_{t_{k}}(t) H_{t_{k}}(\vartheta) I_{k}^{p}\left(y\left(t_{k}\right)\right)
$$

para $\vartheta \in\left[t_{0}-r, t_{0}+\sigma\right]$, não é difícil de mostrar que $G_{p}$ dada por

$$
G_{p}(y, t)=F_{p}(y, t)+J_{p}(y, t)
$$

pertence a $\mathcal{F}(\Omega, h)$, para $p=1,2, \ldots$, onde $\Omega=P C_{1} \times\left[t_{0}-r, t_{0}+\sigma\right] \mathrm{e}$

$$
h(t)=\left\{\begin{array}{l}
\int_{t_{0}}^{t}[M(s)+L(s)] d s+\max \left(K_{1}, K_{2}\right) \sum_{k=0}^{m} H_{t_{k}}(t), t \in\left[t_{0}, t_{0}+\sigma\right] \\
0, t \in\left[t_{0}-r, t_{0}\right] .
\end{array}\right.
$$

De acordo com os resultados apresentados nos Teoremas 3.2 e 3.3, para cada $p=$ 
$0,1, \ldots$, existe uma correspondência biunívoca entre a solução do problema $(2.21)$ e a solução da equação diferencial generalizada

$$
\frac{d x}{d \tau}=D G_{p}(x, t)
$$

com condição inicial $x\left(t_{0}\right)(\vartheta)=\phi_{p}(\vartheta), \vartheta \in\left[t_{0}-r, t_{0}\right]$ e $x\left(t_{0}\right)(\vartheta)=\phi_{p}\left(t_{0}\right), \vartheta \in\left[t_{0}, t_{0}+\sigma\right]$. Assim, estamos aptos a demonstrar o resultado abaixo, já enunciado anteriormente com a finalidade de demonstrar um outro resultado mais adiante (veja o Capítulo 2, última seção).

Teorema 3.4. Suponhamos que para $p=0,1, \ldots, \phi_{p} \in G^{-}\left([-r, 0], \mathbb{R}^{n}\right)$ e $f_{p}$, $I_{k}^{p}$ satisfaçam as condições (A), (B), (A') e (B') enunciadas anteriormente para as mesmas funções $M$ e $L$ e as mesmas constantes $K_{1}$ e $K_{2}$ para todo $p=0,1, \ldots$ Consideremos as relações

$$
\lim _{p \rightarrow \infty} \sup _{\vartheta \in\left[t_{0}, t_{0}+\sigma\right]}\left|\int_{t_{0}}^{\vartheta}\left[f_{p}\left(x_{s}, s\right)-f_{0}\left(x_{s}, s\right)\right] d s\right|=0
$$

para todo $x \in P C_{1} e$

$$
\lim _{p \rightarrow \infty} I_{k}^{p}(x)=I_{k}^{0}(x)
$$

para todo $x \in \mathbb{R}^{n}, k=0,1, \ldots, m$. Suponhamos que $y_{p}:\left[t_{0}-r, t_{0}+\sigma\right] \rightarrow \mathbb{R}^{n}$, para $p=1,2, \ldots$, seja uma solução de problema (3.46) em $\left[t_{0}-r, t_{0}+\sigma\right]$ tal que

$$
\lim _{p \rightarrow \infty} y_{p}(s)=y(s) \text { uniformemente em }\left[t_{0}-r, t_{0}+\sigma\right]
$$

Então y: $\left[t_{0}-r, t_{0}+\sigma\right] \rightarrow \mathbb{R}^{n}$ será uma solução do problema

$$
\left\{\begin{array}{l}
\dot{y}(t)=f_{0}\left(y_{t}, t\right), t \neq t_{k} \\
\Delta y\left(t_{k}\right)=I_{k}^{0}\left(y\left(t_{k}\right)\right), k=0,1, \ldots, m \\
y_{t_{0}}=\phi_{0} .
\end{array}\right.
$$

Prova: Dado $t \in\left[t_{0}-r, t_{0}+\sigma\right]$, sejam

$$
x_{p}(t)(\tau)=\left\{\begin{array}{l}
y_{p}(\vartheta), \vartheta \in\left[t_{0}-r, t\right] \\
y_{p}(t), \vartheta \in\left[t, t_{0}+\sigma\right]
\end{array}\right.
$$

para $p=1,2, \ldots$ e

$$
x(t)(\tau)=\left\{\begin{array}{l}
y(\vartheta), \vartheta \in\left[t_{0}-r, t\right] \\
y(t), \vartheta \in\left[t, t_{0}+\sigma\right] .
\end{array}\right.
$$


Então $x_{p}(t) \in G^{-}\left(\left[t_{0}-r, t_{0}+\sigma\right], \mathbb{R}^{n}\right)$ é uma solução de $(3.51)$ em $\left[t_{0}-r, t_{0}+\sigma\right]$ para $p=1,2, \ldots$ pelo Teorema 3.2 .

Por (3.54) e (3.56), é fácil checar que para $s \in\left[t_{0}, t_{0}+\sigma\right]$ temos

$$
\lim _{p \rightarrow \infty} x_{p}(s)=x(s)
$$

em $G^{-}\left(\left[t_{0}-r, t_{0}+\sigma\right], \mathbb{R}^{n}\right)$ e $x(s) \in P C_{1}$ para $s \in\left[t_{0}, t_{0}+\sigma\right]$.

Por (3.52) e (3.53), podemos mostrar que

$$
\lim _{p \rightarrow \infty} G_{p}(y, t)=G(y, t)
$$

para $(y, t) \in P C_{1} \times\left[t_{0}-r, t_{0}+\sigma\right]$.

Pela Proposição 1.31, $x:\left[t_{0}, t_{0}+\sigma\right] \rightarrow P C_{1}$ é uma solução de

$$
\frac{d x}{d \tau}=D G_{0}(x, t)
$$

Daí, (3.57) implica (3.30) e, então Teorema 3.3 implica que a função $y:\left[t_{0}-r, t_{0}+\sigma\right] \rightarrow \mathbb{R}^{n}$ é uma solução do problema (3.55). A demonstração está, portanto, completa.

Seja $\left[t_{0}, \infty\right) \subset \mathbb{R}$ dada e consideremos as funções $f(\phi, t)$ aplicando $G^{-}\left(\left[t_{0}-r, t_{0}\right], \mathbb{R}^{n}\right) \times$ $\left[t_{0}, \infty\right)$ em $\mathbb{R}^{n}$. Suponhamos que uma sequência $\left\{t_{l}\right\}$ seja tal que $t_{0}<t_{1}<t_{2}<\ldots<t_{l}<$ $\ldots$ e $t_{l} \rightarrow \infty$ quando $l \rightarrow \infty$.

Consideremos que as funções $y:\left[t_{0}-r, \infty\right) \rightarrow \mathbb{R}^{n}$ sejam contínuas à esquerda em seus domínios de definição e admitam limite lateral a direita $y(t+)$ em todo ponto e são tais que $y(t+) \neq y(t)$ somente para $t=t_{l}, l=0,1,2, \ldots$, e a restrição $\left.y\right|_{\left[t_{0}-r, t_{0}\right]}$ pertença a $G^{-}\left(\left[t_{0}-r, t_{0}\right], \mathbb{R}^{n}\right)$. Denotaremos esta família de funções por $G^{-}\left(\left[t_{0}-r, \infty\right)\right)=$ $G^{-}\left(\left[t_{0}-r, \infty\right), \mathbb{R}^{n}\right)$. É claro que para uma função $y$ tendo estas propriedades, temos $y_{t} \in G^{-}\left([-r, 0], \mathbb{R}^{n}\right)$ para $t \in\left[t_{0}, \infty\right)$. Portanto $f\left(y_{t}, t\right):\left[t_{0}, \infty\right) \rightarrow \mathbb{R}^{n}$ está bem definida para $t \in\left[t_{0}, \infty\right)$ e $y:\left[t_{0}-r, \infty\right) \rightarrow \mathbb{R}^{n}$ pertence a classe $G^{-}\left(\left[t_{0}-r, \infty\right)\right)$ de funções apresentadas acima.

Seja $P C_{1} \subset G^{-}\left(\left[t_{0}-r, \infty\right)\right)$ um conjunto aberto (na topologia da convergência localmente uniforme em $\left.G^{-}\left(\left[t_{0}-r, \infty\right)\right)\right)$ com a seguinte propriedade: se $y$ for um elemento de $P C_{1}$ e $\bar{t} \in\left[t_{0}, \infty\right)$, então $\bar{y}$ será dada por

$$
\bar{y}(t)=\left\{\begin{array}{l}
y(t), t_{0}-r \leq t \leq \bar{t} \\
y(\bar{t}+), \bar{t}<t \leq \infty
\end{array}\right.
$$

também será um elemento de $P C_{1}$. 
Para o próximo resultado, iremos supor que para cada $y \in P C_{1}$, a função $f\left(y_{t}, t\right)$ : $\left[t_{0}, \infty\right) \rightarrow \mathbb{R}^{n}$ é lo calmente Lebesgue integrável e, mais ainda, valem as condições seguintes:

$\left(A^{*}\right)$ existe uma função localmente Lebesgue integrável $M(t):\left[t_{0}, \infty\right) \rightarrow \mathbb{R}$ tal que para todo $x \in P C_{1}$ e todo $u_{1}, u_{2} \in\left[t_{0},+\infty\right)$,

$$
\left|\int_{u_{1}}^{u_{2}} f\left(x_{s}, s\right) d s\right| \leq \int_{u_{1}}^{u_{2}} M(s) d s
$$

$\left(B^{*}\right)$ existe uma função localmente Lebesgue integrável $L:\left[t_{0}, \infty\right) \rightarrow \mathbb{R}$ tal que para todo $x, y \in P C_{1}$ e todo $u_{1}, u_{2} \in\left[t_{0},+\infty\right)$,

$$
\left|\int_{u_{1}}^{u_{2}}\left[f\left(x_{s}, s\right)-f\left(y_{s}, s\right)\right] d s\right| \leq \int_{u_{1}}^{u_{2}} L(s)\left\|x_{s}-y_{s}\right\| d s .
$$

Para as funções impulsivas $I_{i}: \mathbb{R}^{n} \rightarrow \mathbb{R}^{n}, i=0,1,2, \ldots$, assumiremos as seguintes condições:

$\left(A^{\prime *}\right)$ existe uma constante $K_{1}>0$ tal que para todo $i=0,1,2, \ldots$ e todo $x \in \mathbb{R}^{n}$,

$$
\left|I_{i}(x)\right| \leq K_{1}
$$

$\left(B^{\prime *}\right)$ existe uma constante $K_{2}>0$ tal que para todo $i=0,1,2, \ldots$ e todo $x, y \in \mathbb{R}^{n}$,

$$
\left|I_{i}(x)-I_{i}(y)\right| \leq K_{2}|x-y|
$$

Consideremos, para cada $(y, t) \in P C_{1} \times\left[t_{0}-r, \infty\right)$ e cada $k=0,1,2, \ldots$, que

$$
F_{k}(y, t)(\vartheta)=\left\{\begin{array}{l}
0, \text { se } t_{0}-r \leq \vartheta \leq t_{0} \text { ou } t_{0}-r \leq t \leq t_{0} \\
\int_{t_{0}}^{\vartheta} f_{k}\left(y_{s}, s\right) d s, \text { se } t_{0} \leq \vartheta \leq t<\infty \\
\int_{t_{0}}^{t} f_{k}\left(y_{s}, s\right) d s, \text { se } t_{0} \leq t \leq \vartheta<\infty
\end{array}\right.
$$

onde $\vartheta \in\left[t_{0}-r, \infty\right)$ e $f_{k}$ satisfaz $\left(A^{*}\right)$ e $\left(B^{*}\right)$ para $k=0,1,2, \ldots$

Consideramos também

$$
J_{k}(y, t)(\vartheta)=\sum_{i=1}^{\infty} H_{t_{i}}(t) H_{t_{i}}(\vartheta) I_{i}^{k}\left(y\left(t_{i}\right)\right)
$$


onde $\vartheta \in\left[t_{0}-r, \infty\right)$ e $I_{i}^{k}$ satisfaz as condições $\left(A^{\prime *}\right)$ e $\left(B^{\prime *}\right)$ para $k=0,1,2, \ldots$ e $i=0,1,2, \ldots$.

Então $F_{k} \in \mathcal{F}\left(\Omega, h_{1}\right)$ e $J_{k} \in \mathcal{F}\left(\Omega, h_{2}\right), k=0,1,2, \ldots$, onde

$$
h_{1}(t)=\left\{\begin{array}{l}
\int_{t_{0}}^{t}[M(s)+L(s)] d s, t \in\left[t_{0},+\infty\right) . \\
0, t \in\left[t_{0}-r, t_{0}\right]
\end{array}\right.
$$

$\mathrm{e}$

$$
h_{2}(t)=\max \left(K_{1}, K_{2}\right) \sum_{i=0}^{\infty} H_{t_{i}}(t), t \in\left[t_{0}-r, \infty\right) .
$$

Portanto, se para cada $k=0,1,2, \ldots$, definirmos

$$
G_{k}(y, t)=F_{k}(y, t)+J_{k}(y, t)
$$

para $(y, t) \in P C_{1} \times\left[t_{0}-r, \infty\right)$, teremos $G_{k} \in \mathcal{F}(\Omega, h)$, onde $h=h_{2}+h_{1}$ a qual é claramente contínua à esquerda e não decrescente.

O próximo resultado é uma generalização do Teorema 8.6 de [26], para uma classe especial de equações diferenciais generalizadas que aplica valores em $G^{-}\left(\left[t_{0}-r, \infty\right)\right)$. Os Teoremas que dão a correspondência entre a solução de certa EDOG com a solução de uma EDFRI e vice-versa foram cruciais na demonstração (Teoremas 3.2 e 3.3).

Teorema 3.5. Suponhamos que, para $k=0,1, \ldots, G_{k}: P C_{1} \times\left[t_{0}-r, \infty\right) \rightarrow G^{-}\left(\left[t_{0}-r, \infty\right)\right)$ seja dada como em (3.64) e que tenhamos

$$
\lim _{k \rightarrow \infty} F_{k}(y, t)=F_{0}(y, t) \quad e \quad \lim _{k \rightarrow \infty} J_{k}(y, t)=J_{0}(y, t)
$$

para $(y, t) \in P C_{1} \times\left[t_{0}-r, \infty\right)$. Seja $x:\left[t_{0}-r, \infty\right) \rightarrow P C_{1}$ a única solução da equação diferencial generalizada

$$
\frac{d x}{d \tau}=D G_{0}(x, t)=D\left[F_{0}(x, t)+J_{0}(x, t)\right]
$$

com a condição inicial $x\left(t_{0}\right) \in P C_{1}$ dada por

$$
x\left(t_{0}\right)(\vartheta)=\left\{\begin{array}{l}
\phi\left(\vartheta-t_{0}\right), t_{0}-r \leq \vartheta \leq t_{0}, \\
\phi(0), t_{0} \leq \vartheta<\infty,
\end{array}\right.
$$

onde $\phi \in G^{-}\left([-r, 0], \mathbb{R}^{n}\right)$. Seja $\left\{\phi_{k}\right\}_{k \geq 1} \in G^{-}\left([-r, 0], \mathbb{R}^{n}\right)$ uma sequência que converge uniformemente para uma função $\phi \in G^{-}\left([-r, 0], \mathbb{R}^{n}\right)$. Então existe um inteiro positivo 
$m$ tal que, para cada $k>m$, existe uma solução $x_{k}:\left[t_{0}-r, \infty\right) \rightarrow P C_{1}$ da equação diferencial generalizada

$$
\frac{d x}{d \tau}=D G_{k}(x, t)
$$

em $\left[t_{0}-r, \infty\right)$, com condição inicial

$$
x_{k}\left(t_{0}\right)(\vartheta)=\left\{\begin{array}{l}
\phi_{k}\left(\vartheta-t_{0}\right), \quad t_{0}-r \leq \vartheta \leq t_{0}, \\
\phi_{k}(0), \quad t_{0} \leq \vartheta<\infty,
\end{array}\right.
$$

$e$

$$
\lim _{k \rightarrow+\infty} x_{k}(s)=x(s), \quad s \in\left[t_{0}-r, \infty\right)
$$

Prova: Por (3.65), está claro que para $\vartheta \in\left[t_{0}-r, \infty\right)$, temos

$$
\lim _{k \rightarrow \infty} F_{k}(y, t)(\vartheta)=F_{0}(y, t)(\vartheta) \quad \text { e } \quad \lim _{k \rightarrow \infty} J_{k}(y, t)(\vartheta)=J_{0}(y, t)(\vartheta)
$$

Portanto, por (3.61) e (3.62), também temos

$$
\lim _{k \rightarrow \infty} \int_{t_{0}}^{\vartheta} f_{k}\left(y_{s}, s\right) d s=\int_{t_{0}}^{\vartheta} f_{0}\left(y_{s}, s\right) d s, \quad \vartheta \in\left[t_{0}, \infty\right)
$$

e

$$
\lim _{k \rightarrow \infty} I_{i}^{k}\left(y\left(t_{i}\right)\right)=I_{i}^{0}\left(y\left(t_{i}\right)\right), \quad i=0,1,2, \ldots
$$

Seja $x:\left[t_{0}-r, \infty\right) \rightarrow P C_{1}$ a única solução da equação diferencial generalizada (3.66), com condição inicial (3.67). Definamos $y:\left[t_{0}-r, \infty\right) \rightarrow \mathbb{R}^{n}$ como em (3.30). Então, pelo Teorema 3.3, y será uma solução de (2.13) em $\left[t_{0}-r, \infty\right)$. Note que, como $\phi_{k}\left(\vartheta-t_{0}\right) \rightarrow x\left(t_{0}\right)(\vartheta)=y(\vartheta)$ uniformemente em $\left[t_{0}-r, t_{0}\right]$, quando $k \rightarrow \infty$, então $\phi_{k} \rightarrow y_{t_{0}}$ uniformemente em [-r,0]. Portanto, o Teorema 2.31 implica que, para $k \in \mathbb{N}$ suficientemente grande, isto é, para $k>k_{1}$, existe uma solução $y_{k}$ da equação

$$
\left\{\begin{array}{l}
\dot{y}=f_{k}\left(y_{t}, t\right), t \neq t_{i} \\
\Delta y\left(t_{i}\right)=I_{i}^{k}\left(y\left(t_{i}\right)\right), i=0,1,2 \ldots \\
y_{t_{0}}=\phi_{k}
\end{array}\right.
$$

$\mathrm{em}\left[t_{0}-r, \infty\right)$ e $y_{k}(s) \rightarrow y(s), s \in\left[t_{0}-r, \infty\right)$ (em particular, $y_{k}(\theta) \rightarrow y(\theta)$, uniformemente em $[-r, 0]$, por hipótese). Portanto, se para cada $k=1,2, \ldots$, definirmos

$$
x_{k}(t)(\vartheta)= \begin{cases}y_{k}(\vartheta), & t_{0}-r \leq \vartheta \leq t \\ y_{k}(t), & t \leq \vartheta<\infty\end{cases}
$$


onde $t \in\left[t_{0}-r, \infty\right)$, então o Teorema 3.3 implicará que, para $k>k_{1}, x_{k}(t) \in P C_{1}$ é uma solução de (3.68), com condição inicial

$$
x_{k}\left(t_{0}\right)(\vartheta)=\left\{\begin{array}{l}
\phi_{k}\left(\vartheta-t_{0}\right), \quad t_{0}-r \leq \vartheta \leq t_{0}, \\
\phi_{k}(0), \quad t_{0} \leq \vartheta<\infty
\end{array}\right.
$$

Também, para todo $t \in\left[t_{0}-r, \infty\right)$ fixo, temos, pela definição,

$$
\left\|x_{k}(t)-x_{0}(t)\right\|=\sup _{\vartheta \in\left[t_{0}-r, \infty\right)}\left|x_{k}(t)(\vartheta)-x_{0}(t)(\vartheta)\right|=\sup _{\vartheta \in\left[t_{0}-r, t\right]}\left|y_{k}(\vartheta)-y(\vartheta)\right|
$$

Então, como $\lim _{k \rightarrow \infty} y_{k}(s)=y(s), s \in\left[t_{0}-r, t\right]$, segue que para todo $\varepsilon>0$, existirá $k_{2}=k_{2}(t) \in \mathbb{N}$ tal que para $k>k_{2}$,

$$
\sup _{\vartheta \in\left[t_{0}-r, t\right]}\left|y_{k}(\vartheta)-y(\vartheta)\right|<\varepsilon
$$

Portanto,

$$
\left\|x_{k}(t)-x_{0}(t)\right\| \leq \varepsilon, \quad k>k_{2} .
$$

Isto implicará que $\lim _{k \rightarrow \infty} x_{k}(t)=x_{0}(t)$. Daí, tomando $m>\max \left\{k_{1}, k_{2}\right\}$, teremos o resultado desejado.

Para o próximo resultado, consideraremos $\Omega=P C_{1} \times\left[t_{0}-r, \infty\right)$. Consideraremos, também, que para cada $(y, t) \in \Omega$ e cada $k=0,1,2, \ldots, F_{k}(y, t)$ é definida como em (3.61) e $J_{k}(y, t)$ é definida como em (3.62). Mas, ao contrário do que foi assumido em (3.61) e (3.62), consideraremos que para cada $k=1,2, \ldots, f_{k}$ e $I_{k}$ satisfazem as condições do tipo $\left(A^{*}\right),\left(B^{*}\right),\left(A^{*}\right)$ e $\left(B^{\prime *}\right)$, porém para diferentes funções $M$ e $L$ e diferentes valores $K_{1}$ e $K_{2}$. Assim, vamos supor que, para cada $k=0,1,2, \ldots, f_{k}: G^{-}([-r, 0],) \rightarrow \mathbb{R}^{n}$ é tal que $t \mapsto f_{k}\left(y_{t}, t\right)$ é localmente Lebesgue integrável, para $y \in P C_{1}$, e valem as condições:

$\left(A_{k}{ }^{*}\right)$ existe uma função localmente Lebesgue integrável $M_{k}(t):\left[t_{0}, \infty\right) \rightarrow \mathbb{R}$ tal que para todo $x \in P C_{1}$ e todo $u_{1}, u_{2} \in\left[t_{0},+\infty\right)$,

$$
\left|\int_{u_{1}}^{u_{2}} f_{k}\left(x_{s}, s\right) d s\right| \leq \int_{u_{1}}^{u_{2}} M_{k}(s) d s
$$

$\left(B_{k}{ }^{*}\right)$ existe uma função localmente Lebesgue integrável $L_{k}:\left[t_{0}, \infty\right) \rightarrow \mathbb{R}$ tal que para todo $x, y \in P C_{1}$ e todo $u_{1}, u_{2} \in\left[t_{0},+\infty\right)$,

$$
\left|\int_{u_{1}}^{u_{2}}\left[f_{k}\left(x_{s}, s\right)-f_{k}\left(y_{s}, s\right)\right] d s\right| \leq \int_{u_{1}}^{u_{2}} L_{k}(s)\left\|x_{s}-y_{s}\right\| d s .
$$


Para cada $k=0,1,2, \ldots$, consideramos que os operadores de impulsos $I_{i}^{k}: \mathbb{R}^{n} \rightarrow \mathbb{R}^{n}$, $i=0,1,2, \ldots$, satisfazem as seguintes condições:

$\left(A_{k}^{\prime *}\right)$ existe uma constante $K_{1}^{k}>0$ tal que para todo $i=0,1,2, \ldots$ e todo $x \in \mathbb{R}^{n}$,

$$
\left|I_{i}^{k}(x)\right| \leq K_{1}^{k}
$$

$\left(B_{k}^{\prime *}\right)$ existe uma constante $K_{2}^{k}>0$ tal que para todo $i=0,1,2, \ldots$ e todo $x, y \in \mathbb{R}^{n}$,

$$
\left|I_{i}^{k}(x)-I_{i}^{k}(y)\right| \leq K_{2}^{k}|x-y|
$$

Daí, definindo

$$
h_{1}^{k}(t)=\left\{\begin{array}{l}
\int_{t_{0}}^{t}\left[M_{k}(s)+L_{k}(s)\right] d s, t \in\left[t_{0},+\infty\right) . \\
0, t \in\left[t_{0}-r, t_{0}\right]
\end{array}\right.
$$

e

$$
h_{2}^{k}(t)=\max \left(K_{1}^{k}, K_{2}^{k}\right) \sum_{i=0}^{\infty} H_{t_{i}}(t), t \in\left[t_{0}-r, \infty\right) .
$$

temos $F_{k} \in \mathcal{F}\left(\Omega, h_{1}^{k}\right)$ e $J_{k} \in \mathcal{F}\left(\Omega, h_{2}^{k}\right)$, para $k=0,1,2, \ldots$, onde

$$
F_{k}(y, t)(\vartheta)=\left\{\begin{array}{l}
0, \text { se } t_{0}-r \leq \vartheta \leq t_{0} \text { ou } t_{0}-r \leq t \leq t_{0} \\
\int_{t_{0}}^{\vartheta} f_{k}\left(y_{s}, s\right) d s, \text { se } t_{0} \leq \vartheta \leq t<\infty \\
\int_{t_{0}}^{t} f_{k}\left(y_{s}, s\right) d s, \text { se } t_{0} \leq t \leq \vartheta<\infty
\end{array}\right.
$$

e

$$
J_{k}(y, t)(\vartheta)=\sum_{i=1}^{\infty} H_{t_{i}}(t) H_{t_{i}}(\vartheta) I_{i}^{k}\left(y\left(t_{i}\right)\right)
$$

onde $(y, t) \in P C_{1} \times\left[t_{0}-r, \infty\right), \vartheta \in\left[t_{0}-r, \infty\right)$ e $f_{k}$ e $I_{i}^{k}$ satisfazem as condições $\left(A_{k}{ }^{*}\right)$, $\left(B_{k}^{*}\right),\left(A_{k}^{\prime *}\right)$ e $\left(B_{k}^{*}\right)$ para $k=0,1,2, \ldots$ e $i=0,1,2, \ldots$

Logo, definindo

$$
G_{k}(y, t)=F_{k}(y, t)+J_{k}(y, t)
$$

onde $(y, t) \in \Omega$ e $k=0,1,2, \ldots$, e fazendo

$$
h_{k}(t)=h_{1}^{k}(t)+h_{2}^{k}(t)
$$


para $t \in\left[t_{0}-r, \infty\right)$, temos $G_{k} \in \mathcal{F}\left(\Omega, h_{k}\right)$, para $k=0,1,2, \ldots$

O próximo resultado é bem interessante e é ainda mais geral do que o Teorema 3.5. Ele será crucial para a demonstração do nosso resultado sobre o Princípio da Média para EDFRIs, pois o parâmetro pequeno $\varepsilon$ da EDFRI média, quando fazemos $\varepsilon \rightarrow 0^{+}$, será relacionado inversamente com a sequência de índices $k$, quando $k \rightarrow \infty$, das funções $f_{k} \mathrm{e}$ $I_{i}^{k}$

Teorema 3.6. Suponhamos que, para $k=0,1,2, \ldots, G_{k}: \Omega \rightarrow G^{-}\left(\left[t_{0}-r, \infty\right)\right)$ seja dada como em (3.70). Então, para $k=0,1,2, \ldots, G_{k} \in \mathcal{F}\left(\Omega, h_{k}\right)$, onde $h_{k}$ é dada por (3.71) e é uma função não decrescente e contínua à esquerda. Suponhamos que, para cada $(y, t) \in \Omega$, tenhamos

$$
F_{0}(y, t)=\lim _{k \rightarrow \infty} F_{k}(y, t) \quad e \quad J_{0}(y, t)=\lim _{k \rightarrow \infty} J_{k}(y, t)
$$

Além disso, suponhamos que $h_{0}:\left[t_{0}-r, \infty\right) \rightarrow \mathbb{R}$ seja contínua $e$

$$
\limsup _{k \rightarrow \infty}\left[h_{k}\left(t_{2}\right)-h_{k}\left(t_{1}\right)\right] \leq h_{0}\left(t_{2}\right)-h_{0}\left(t_{1}\right)
$$

para $t_{0}-r \leq t_{1} \leq t_{2}<\infty$. Seja $x:\left[t_{0}-r, \infty\right) \rightarrow P C_{1}$ uma solução da equação diferencial generalizada

$$
\frac{d x}{d \tau}=D G_{0}(x, t)
$$

em $\left[t_{0}-r, \infty\right]$ e suponhamos que exista um $\rho>0$ tal que se $s \in\left[t_{0}-r, \infty\right)$ e $\|y-x(s)\|<\rho$, então $(y, s) \in \Omega$. Consideremos uma sequência $\left\{y_{k}\right\}_{k \in \mathbb{N}}$ em $P C_{1}$ tal que

$$
\lim _{k \rightarrow \infty} y_{k}=x\left(t_{0}\right)
$$

Então para todo $\mu>0$ e todo $\sigma>0$, existirá um $k_{*} \in \mathbb{N}$ tal que para $k \in \mathbb{N}, k>k_{*}$, existirá uma solução $x_{k}$ da equação diferencial generalizada

$$
\frac{d x}{d \tau}=D G_{k}(x, t)
$$

$e m\left[t_{0}-r, \infty\right) \operatorname{com} x_{k}\left(t_{0}\right)=y_{k} e$

$$
\left\|x_{k}(s)-x(s)\right\|<\mu, \quad \text { para } s \in\left[t_{0}-r, t_{0}+\sigma\right]
$$

Prova: Por (3.72), temos

$$
\lim _{k \rightarrow \infty} G_{k}(y, t)=G_{0}(y, t), \quad(y, t) \in \Omega
$$


Daí, (3.78) e (3.75) implicam que existe $k_{1} \in \mathbb{N}$ tal que pata $k>k_{1}$, valem

$$
\left\|G_{k}\left(x\left(t_{0}\right), t_{0}+\right)-G_{k}\left(x\left(t_{0}\right), t_{0}\right)-G_{0}\left(x\left(t_{0}\right), t_{0}+\right)+G_{0}\left(x\left(t_{0}\right), t_{0}\right)\right\|<\frac{\rho}{2}
$$

e

$$
\left\|y_{k}-x\left(t_{0}\right)\right\|<\frac{\rho}{2}
$$

Logo, para $k>k_{1}$, temos $\left(y_{k}, t_{0}\right) \in \Omega \mathrm{e}$

$$
\begin{gathered}
\left\|y_{k}+G_{k}\left(y_{k}, t_{0}+\right)-G_{k}\left(y_{k}, t_{0}\right)-x\left(t_{0}\right)\right\| \leq\left\|y_{k}-x\left(t_{0}\right)\right\|+ \\
+\left\|G_{k}\left(y_{k}, t_{0}+\right)-G_{k}\left(x\left(t_{0}\right), t_{0}+\right)-G_{k}\left(y_{k}, t_{0}\right)+G_{k}\left(x\left(t_{0}\right), t_{0}\right)\right\|+ \\
+\left\|G_{k}\left(x\left(t_{0}\right), t_{0}+\right)-G_{k}\left(x\left(t_{0}\right), t_{0}\right)-G_{0}\left(x\left(t_{0}\right), t_{0}+\right)+G_{0}\left(x\left(t_{0}\right), t_{0}\right)\right\|+ \\
+\left\|G_{0}\left(x\left(t_{0}\right), t_{0}+\right)-G_{0}\left(x\left(t_{0}\right), t_{0}\right)\right\|< \\
<\frac{\rho}{2}+\left\|y_{k}-x\left(t_{0}\right)\right\|\left[h_{k}\left(t_{0}+\right)-h_{k}\left(t_{0}\right)\right]+\frac{\rho}{2}+\left[h_{0}\left(t_{0}+\right)-h_{0}\left(t_{0}\right)\right]<
\end{gathered}
$$

onde usamos (1.16) para $G_{k}$ e (1.15) para $G_{0}$.

Mas por (3.73) e pela continuidade de $h_{0}$, temos

$$
\frac{\rho}{2}+\left\|y_{k}-x\left(t_{0}\right)\right\|\left[h_{k}\left(t_{0}+\right)-h_{k}\left(t_{0}\right)\right]+\frac{\rho}{2}+\left[h_{0}\left(t_{0}+\right)-h_{0}\left(t_{0}\right)\right]<\rho .
$$

Logo,

$$
\left\|y_{k}+G_{k}\left(y_{k}, t_{0}+\right)-G_{k}\left(y_{k}, t_{0}\right)-x\left(t_{0}\right)\right\|<\rho
$$

e, portanto, por hipótese,

$$
\left(y_{k}+G_{k}\left(y_{k}, t_{0}+\right)-G_{k}\left(y_{k}, t_{0}\right), t_{0}\right) \in \Omega, \quad \text { para } k>k_{1} .
$$

Assim, para $k>k_{1}$, estamos nas condições do teorema de existência e unicidade (Teorema 1.28). Então, para $k>k_{1}$, existem $d>0$ e uma solução $x_{k}:\left[t_{0}-r, \infty\right) \rightarrow P C_{1}$ de (3.76) em $\left[t_{0}-r, t_{0}+d\right]$ tal que $x_{k}\left(t_{0}\right)=y_{k}, k>k_{1}$.

Queremos provar que $\lim _{k \rightarrow \infty} x_{k}(t)=x(t)$, para $t \in\left[t_{0}-r, t_{0}+d\right]$. Note que, por (3.75) e pelo parágrafo anterior, basta provarmos que $\lim _{k \rightarrow \infty} x_{k}(t)=x(t)$, para $t \in\left[t_{0}, t_{0}+d\right]$.

Para $k>k_{1}$ e $\vartheta \in\left[t_{0}-r, \infty\right)$, seja

$$
y_{k}(\vartheta)=\left\{\begin{array}{l}
x_{k}\left(t_{0}\right)(\vartheta), t_{0}-r \leq \vartheta \leq t_{0} \\
x_{k}(\vartheta)(\vartheta), t_{0} \leq \vartheta<\infty
\end{array}\right.
$$


Então, pelo Teorema 3.3, $y_{k}$ é solução de

$$
\left\{\begin{array}{l}
\dot{y}(t)=f_{k}\left(y_{t}, t\right), t \neq t_{i} \\
\Delta y\left(t_{i}\right)=I_{i}^{k}\left(y\left(t_{i}\right)\right), i=0,1, \ldots \\
y_{t_{0}}=\phi_{k}
\end{array}\right.
$$

em $\left[t_{0}-r, t_{0}+d\right]$, onde definimos $\phi_{k}\left(\vartheta-t_{0}\right)=y_{k}(\vartheta), t_{0}-r \leq \vartheta \leq t_{0}$. Logo, para $k>k_{1}$, $x_{k}\left(t_{0}\right)$ pode ser descrita como

$$
x_{k}\left(t_{0}\right)(\vartheta)=\left\{\begin{array}{l}
\phi_{k}\left(\vartheta-t_{0}\right), t_{0}-r \leq \vartheta \leq t_{0} \\
\phi_{k}(0), t_{0} \leq \vartheta<\infty
\end{array}\right.
$$

onde $\phi_{k} \in G^{-}\left([-r, 0], \mathbb{R}^{n}\right)$ é definida como acima.

Por (3.75), para $\vartheta \in\left[t_{0}-r, \infty\right)$, temos $\lim _{k \rightarrow \infty} y_{k}(\vartheta)=x\left(t_{0}\right)(\vartheta)$. Então, definindo $\phi_{0}\left(\vartheta-t_{0}\right)=x\left(t_{0}\right)(\vartheta)$, temos $\lim _{k \rightarrow \infty} \phi_{k}(\theta)=\phi_{0}(\theta), \theta \in[-r, 0]$.

Agora, definindo $y:\left[t_{0}-r, \infty\right) \rightarrow \mathbb{R}^{n}$ por

$$
y(\vartheta)=\left\{\begin{array}{l}
x\left(t_{0}\right)(\vartheta), t_{0}-r \leq \vartheta \leq t_{0} \\
x(\vartheta)(\vartheta), t_{0} \leq \vartheta<\infty
\end{array}\right.
$$

para $\vartheta \in\left[t_{0}-r, \infty\right)$, temos $y_{t_{0}}=\phi$ e, além disso, pelo Teorema 3.3, y é solução de

$$
\left\{\begin{array}{l}
\dot{y}(t)=f_{0}\left(y_{t}, t\right), t \neq t_{i} \\
\Delta y\left(t_{i}\right)=I_{i}^{0}\left(y\left(t_{i}\right)\right), i=0,1, \ldots \\
y_{t_{0}}=\phi_{0}
\end{array}\right.
$$

em $\left[t_{0}-r, \infty\right)$.

Afirmamos que

$$
\lim _{k \rightarrow \infty} y_{k}(s)=y(s), \quad \text { para } s \in\left[t_{0}-r, \infty\right) .
$$

Por (3.73), segue de maneira análoga ao que foi provado no Teorema 2.29, o fato de que a sequência $\left\{y_{k}\right\}, k>k_{1}$, de funções em $\left[t_{0}, t_{0}+d\right]$ é equilimitada e uniformemente de variação limitada. Portanto, pelo Princípio da Escolha de Helly, a sequência $\left\{y_{k}\right\}, k>k_{1}$, admite uma subsequência pontualmente convergente e, portanto, $y(t)$ é o único ponto de acumulação da sequência $y_{k}(t)$ para todo $t \in\left[t_{0}, t_{0}+d\right]$. E como $\phi_{k} \rightarrow \phi_{0}$, então temos a convergência de $(3.84)$ em $\left[t_{0}-r, t_{0}+d\right]$.

Vamos assumir que o resultado de convergência de (3.84) não acontece em todo o intervalo $\left[t_{0}-r, \infty\right)$. Então, existe um $d^{\prime}, 0<d^{\prime}<\infty$, tal que para todo $d<d^{\prime}$ e 
para $k>k_{1}$, existe uma solução $y_{k}$ de $(3.81)$ em $\left[t_{0}-r, t_{0}+d\right]$, com $\left(y_{k}\right)_{t_{0}}=\phi_{k}$, e $\lim _{p \rightarrow \infty} y_{k}(t)=y(t)$ para $t \in\left[t_{0}-r, t_{0}+d\right]$, mas isto não acontece em $\left[t_{0}-r, t_{0}+d\right]$, para $d>d^{\prime}$.

Pela prova do Teorema 2.28 e por (3.73), temos

$$
\left|y_{k}\left(s_{2}\right)-y_{k}\left(s_{1}\right)\right|<\left|h_{k}\left(s_{2}\right)-h_{k}\left(s_{1}\right)\right| \leq\left|h_{0}\left(s_{2}\right)-h_{0}\left(s_{1}\right)\right| \text {, }
$$

para todo $s_{2}, s_{1} \in\left[t_{0}-r, t_{0}+d^{\prime}\right)$ e todo $k>k_{1}$. Portanto os limites

$$
y_{k}\left(\left(t_{0}+d^{\prime}\right)-\right)=\lim _{\varepsilon \rightarrow 0^{-}} y_{k}\left(t_{0}+d^{\prime}+\varepsilon\right), \quad k>k_{1}
$$

existem e $y_{k}\left(\left(t_{0}+d^{\prime}\right)-\right)=y\left(t_{0}+d^{\prime}\right)$, para $k>k_{1}$, já que $y$ é contínua à esquerda.

Definindo $y_{k}\left(t_{0}+d^{\prime}\right)=y_{k}\left(\left(t_{0}+d^{\prime}\right)-\right)$, para $k>k_{1}$, então $\lim _{k \rightarrow \infty} y_{k}\left(t_{0}+d^{\prime}\right)=y\left(t_{0}+d^{\prime}\right)$. Portanto vale a convergência de (3.84) em $\left[t_{0}-r, t_{0}+d^{\prime}\right]$. Daí, usando $t_{0}+d^{\prime}$ como ponto inicial, podemos provar analogamente que o teorema acontece no intervalo $\left[t_{0}+d^{\prime}, t_{0}+\right.$ $d^{\prime}+\eta$ ], para algum $\eta>0$, e isto contradiz nossa hipótese. Logo a convergência de (3.84) é válida em todo o intervalo $\left[t_{0}-r, \infty\right)$.

Agora, estendendo a definição de $x_{k}, k>k_{1}$, para o intervalo $\left[t_{0}-r, \infty\right)$ da seguinte forma

$$
x_{k}(t)(\vartheta)=\left\{\begin{array}{l}
y_{k}(\vartheta), t_{0}-r \leq \vartheta \leq t \\
y_{k}(t), t \leq \vartheta<\infty
\end{array}\right.
$$

onde $t, \vartheta \in\left[t_{0}-r, \infty\right)$, segue pelo Teorema 3.2 o fato de que $x_{k}(t) \in G^{-}\left(\left[t_{0}-r, \infty\right)\right)$ é uma solução de $(3.76)$ em $\left[t_{0}-r, \infty\right)$. Segue, portanto, da definição de $x_{k}, k>k_{1}$, acima, da definição de $x$ e de (3.84), o fato de que

$$
\lim _{k \rightarrow \infty} x_{k}(s)=x(s), \quad \text { para } s \in\left[t_{0}-r, \infty\right)
$$

Nosso próximo passo nesta demonstração será mostrar que vale (3.77). Para isto, vamos considerar a diferença $x_{k}(s)-x(s)$ para $k \in \mathbb{N}$ suficientemente grande e para $s \in\left[t_{0}-r, \infty\right)$.

Pela definição de uma solução, temos

$$
x_{k}(s)-x(s)=y_{k}-x\left(t_{0}\right)+\int_{t_{0}}^{s} D\left[G_{k}\left(x_{k}(\tau), t\right)-G_{0}(x(\tau), t)\right]
$$

para todo $s \in\left[t_{0}-r, \infty\right)$.

Seja um $\eta>0$ arbitrário dado. Escolhamos um $\tau>0$ tal que $\tau<\eta$ e consideremos a 
partição

$$
A=\left\{\alpha_{0}, \tau_{1}, \alpha_{1}, \ldots, \alpha_{l-1}, \tau_{l}, \alpha_{l}\right\}
$$

de $\left[t_{0}-r, t_{0}+\sigma\right], \sigma>0$, tal que

$$
h_{0}\left(\alpha_{m}\right)-h_{0}\left(\alpha_{m-1}\right)<\tau, \quad \alpha_{m-1}=\tau_{m}<\alpha_{m}, \quad m=1,2, \ldots, l .
$$

É evidente que uma tal partição $A$ existe, pois a função $h_{0}$ é uniformemente contínua em $\left[t_{0}-r, t_{0}+\sigma\right]$. A escolha de $\tau$ também nos dá

$$
\begin{gathered}
h_{0}\left(t_{0}-r\right)-h_{0}\left(\alpha_{m-1}\right)<\tau<\eta, \\
m=1,2, \ldots, l
\end{gathered}
$$

Pela continuidade de $h_{0}$, temos $D=D\left(\eta, h_{0},\left[t_{0}-r, t_{0}+\sigma\right]\right)=\emptyset$ e a partição $A$ satisfaz (1.34), (1.35) e (1.36). Como (3.73) está satisfeito, existe um $k_{0} \in \mathbb{N}$ tal que para $k>k_{0}$, $k \in \mathbb{N}$, temos

$$
h_{k}\left(\alpha_{m}\right)-h_{k}\left(\alpha_{m-1}\right)<\tau, m=1,2, \ldots, l .
$$

Consequentemente,

$$
h_{k}\left(\alpha_{m}\right)-h_{k}\left(\alpha_{m-1}\right)<\eta, m=1,2, \ldots, l .
$$

Além disso, podemos tomar $\tau_{m}=\alpha_{m-1}, m=1,2, \ldots, l$ e a desigualdade

$$
h_{k}\left(\alpha_{m-1}+\right)-h_{k}\left(\alpha_{m-1}\right)<h_{k}\left(\alpha_{m}\right)-h_{k}\left(\alpha_{m-1}\right)<\tau
$$

implica que

$$
h_{k}\left(\tau_{m}+\right)-h\left(\tau_{m}\right)<\tau<\eta
$$

e, portanto, $\tau_{m}=\alpha_{m-1} \notin D\left(\eta, h_{k},\left[t_{0}-r, t_{0}+\sigma\right]\right)$ para $m=1,2, \ldots, l$. Portanto, para $k>k_{0}$, a partição $A$ satisfaz (1.34), (1.35) e (1.36) com $h_{k}$ ao invés de $h_{0}$. Da convergência ponto a ponto de soluções $x_{k}$ a $x$ existe um $k_{1}>k_{0}, k_{1} \in \mathbb{N}$, tal que para todo $k>k_{1}$ e $m=1,2, \ldots, l$, temos

$$
\left\|x_{k}\left(\tau_{m}\right)-x\left(\tau_{m}\right)\right\|<\tau<\eta
$$

Suponhamos, agora, que $s \in\left(t_{0}-r, t_{0}+\sigma\right]$ seja dado. Então existe um $p \in 1,2, \ldots, l$ tal que $s \in\left(\alpha_{p-1}, \alpha_{p}\right]$. Denotando

$$
A_{s}=\left\{\alpha_{0}, \tau_{1}, \alpha_{1}, \ldots, \alpha_{p-1}, \tau_{p}, \alpha_{p}=\gamma\right\}
$$

obtemos uma partição de $\left[t_{0}-r, \gamma\right]$ e esta partição satisfaz (1.34), (1.35) e (1.36) e também 
satisfaz as mesmas relações com $h_{k}$ ao invés de $h_{0}$, contanto que $k>k_{1}$. Consideraremos a integral

$$
\int_{t_{0}-r}^{s} D\left[G_{k}\left(x_{k}(\tau), t\right)-G_{0}(x(\tau), t)\right]=\int_{t_{0}}^{s} D\left[G_{k}\left(x_{k}(\tau), t\right)-G_{0}(x(\tau), t)\right] .
$$

Pelas propriedades das soluções de (3.74) e (3.76), temos

$$
\left\|x_{k}\left(t_{2}\right)-x_{k}\left(t_{1}\right)\right\| \leq h_{k}\left(t_{2}\right)-h_{k}\left(t_{1}\right)
$$

e

$$
\left\|x\left(t_{2}\right)-x\left(t_{1}\right)\right\| \leq h_{0}\left(t_{2}\right)-h_{0}\left(t_{1}\right)
$$

para $t_{0}-r \leq t_{1} \leq t_{2} \leq s$ e $k>k_{1}$. Então o Lema 1.32 implica a desigualdade

$$
\left\|\int_{t_{0}-r}^{s} D G_{k}\left(x_{k}(\tau), t\right)-\sum_{m=1}^{p} S_{m, k}\right\| \leq 2 \eta\left(h_{k}(s)-h_{k}(\alpha)\right)
$$

para $k>k_{1}$ onde

$$
S_{m, k}=G_{k}\left(x_{k}\left(\tau_{m}\right), \alpha_{m}\right)-G_{k}\left(x_{k}(\tau), \alpha_{m-1}\right)=G_{k}\left(x_{k}\left(\alpha_{m-1}\right), \alpha_{m}\right)-G_{k}\left(x_{k}\left(\alpha_{m-1}\right), \alpha_{m-1}\right)
$$

para $m=1,2, \ldots, p-1 \mathrm{e}$

$$
S_{p, k}=G_{k}\left(x_{k}\left(\alpha_{p-1}, s\right)-G_{k}\left(x_{k}\left(\alpha_{p-1}, \alpha_{p-1}\right)\right.\right.
$$

e, também,

$$
\left\|\int_{t_{0}-r}^{s} D G_{0}(x(\tau), t)-\sum_{m=1}^{p} S_{m, 0}\right\| \leq 2 \eta\left(h_{0}(s)-h_{0}(\alpha)\right),
$$

onde

$$
S_{m, 0}=G_{0}\left(x\left(\tau_{m}\right), \alpha_{m}\right)-G_{0}\left(x\left(\tau_{m}\right), \alpha_{m-1}\right)=G_{0}\left(x\left(\alpha_{m-1}\right), \alpha_{m}\right)-G_{0}\left(x\left(\alpha_{m-1}\right), \alpha_{m-1}\right)
$$

para $m=1,2, \ldots, p-1 \mathrm{e}$

$$
S_{p, 0}=G_{0}\left(x\left(\alpha_{p-1}\right), s\right)-G_{0}\left(x\left(\alpha_{p-1}, \alpha_{p-1}\right) .\right.
$$

Portanto, para $k>k_{1}$, temos

$$
\left\|\int_{t_{0}}^{s} D\left[G_{k}(x(\tau), t)-G_{0}(x(\tau), t)\right]\right\|=\left\|\int_{\alpha}^{s} D G_{k}\left(x_{k}(\tau), t\right)-\int_{\alpha}^{s} D G_{0}(x(\tau), t)\right\| \leq
$$




$$
\leq \sum_{m=1}^{p}\left\|S_{m, k}-S_{m, 0}\right\|+2 \eta\left(h_{k}(s)-h_{k}(\alpha)+h_{0}(s)-h_{0}(\alpha)\right)=
$$

$$
\begin{gathered}
=\sum_{m=1}^{p-1}\left\|G_{k}\left(x_{k}\left(\alpha_{m-1}\right), \alpha_{m}\right)-G_{k}\left(x_{k}\left(\alpha_{m-1}\right), \alpha_{m-1}\right)-G_{0}\left(x\left(\alpha_{m-1}\right), \alpha_{m}\right)+G_{0}\left(x\left(\alpha_{m-1}\right), \alpha_{m-1}\right)\right\|+ \\
+\left\|G_{k}\left(x_{k}\left(\alpha_{p-1}\right), s\right)-G_{k}\left(x_{k}\left(\alpha_{p-1}\right), \alpha_{p-1}\right)-G_{0}\left(x_{k}\left(\alpha_{p-1}\right), s\right)+G_{k}\left(x_{k}\left(\alpha_{p-1}\right), \alpha_{p-1}\right)\right\|+ \\
+2 \eta\left(h_{k}(s)-h_{k}(\alpha)+h_{0}(s)-h_{0}(\alpha)\right) .
\end{gathered}
$$

Pelas propriedades da classe $\mathcal{F}\left(\Omega, h_{k}\right)$ e por (3.90), para $k>k_{1}$ e $m=1,2, \ldots, p-1$, obtemos

$$
\begin{aligned}
& \quad\left\|G_{k}\left(x_{k}\left(\alpha_{m-1}\right), \alpha_{m}\right)-G_{k}\left(x_{k}\left(\alpha_{m-1}\right), \alpha_{m-1}\right)-G_{0}\left(x\left(\alpha_{m-1}\right), \alpha_{m}\right)+G_{0}\left(x\left(\alpha_{m-1}\right), \alpha_{m-1}\right)\right\| \leq \\
& \leq\left\|G_{k}\left(x_{k}\left(\alpha_{m-1}\right), \alpha_{m}\right)-G_{k}\left(x_{k}\left(\alpha_{m-1}\right), \alpha_{m-1}\right)-G_{k}\left(x\left(\alpha_{m-1}\right), \alpha_{m}\right)+G_{k}\left(x\left(\alpha_{m-1}\right), \alpha_{m-1}\right)\right\|+ \\
& +\left\|G_{k}\left(x\left(\alpha_{m-1}\right), \alpha_{m}\right)-G_{0}\left(x\left(\alpha_{m-1}\right), \alpha_{m}\right)\right\|+\left\|G_{k}\left(x\left(\alpha_{m-1}\right), \alpha_{m-1}\right)+G_{0}\left(x\left(\alpha_{m-1}\right), \alpha_{m-1}\right)\right\| \leq \\
& \leq\left\|x_{k}\left(\alpha_{m-1}\right)-x\left(\alpha_{m-1}\right)\right\|\left(h_{k}\left(\alpha_{m}\right)-h_{k}\left(\alpha_{m-1}\right)+\left\|G_{k}\left(x\left(\alpha_{m-1}\right), \alpha_{m}\right)-G_{0}\left(x\left(\alpha_{m-1}\right), \alpha_{m}\right)\right\|+\right. \\
& \quad+\left\|G_{k}\left(x\left(\alpha_{m-1}\right), \alpha_{m-1}\right)+G_{0}\left(x\left(\alpha_{m-1}\right), \alpha_{m-1}\right)\right\| \leq \eta\left(h_{k}\left(\alpha_{m}\right)-h_{k}\left(\alpha_{m-1}\right)\right)+ \\
& \quad+\left\|G_{k}\left(x\left(\alpha_{m-1}\right), \alpha_{m}\right)-G_{0}\left(x\left(\alpha_{m-1}\right), \alpha_{m}\right)\right\|+\left\|G_{k}\left(x\left(\alpha_{m-1}\right), \alpha_{m-1}\right)+G_{0}\left(x\left(\alpha_{m-1}\right), \alpha_{m-1}\right)\right\|
\end{aligned}
$$

e, similarmente, também obtemos

$$
\begin{gathered}
\left\|G_{k}\left(x_{k}\left(\alpha_{p-1}\right), s\right)-G_{k}\left(x_{k}\left(\alpha_{p-1}\right), \alpha_{p-1}\right)-G_{0}\left(x\left(\alpha_{p-1}\right), s\right)+G_{0}\left(x\left(\alpha_{p-1}, \alpha_{p-1}\right)\right)\right\| \leq \\
\left\|x_{k}\left(\alpha_{p-1}\right)-x\left(\alpha_{p-1}\right)\right\|\left(h_{k}\left(\alpha_{p}\right)-h_{k}\left(\alpha_{p-1}\right)\right)+\left\|G_{k}\left(x\left(\alpha_{p-1}\right), \alpha_{p}\right)-G_{0}\left(x\left(\alpha_{p-1}\right), \alpha_{p}\right)\right\|+ \\
+\left\|G_{k}\left(x\left(\alpha_{p-1}\right), \alpha_{p-1}\right)+G_{0}\left(x\left(\alpha_{p-1}\right), \alpha_{p-1}\right)\right\|+h_{k}\left(\alpha_{p}\right)-h_{k}(s)+h_{0}\left(\alpha_{p}\right)-h_{0}(s) \leq \\
\left.\leq \eta\left(h_{k}\left(\alpha_{p}\right)-h_{k}\left(\alpha_{p-1}\right)\right)+\| G_{k}\left(x\left(\alpha_{p-1}\right), \alpha_{p}\right)\right)-G_{0}\left(x\left(\alpha_{p-1}\right), \alpha_{p}\right) \|+ \\
+\left\|G_{k}\left(x\left(\alpha_{p-1}\right), \alpha_{p-1}\right)-G_{0}\left(x\left(\alpha_{p-1}\right), \alpha_{p-1}\right)\right\|+h_{k}\left(\alpha_{p}\right)-h_{k}(s)+h_{0}\left(\alpha_{p}\right)-h_{0}(s) .
\end{gathered}
$$

Portanto (3.88), (3.89), (3.90), (3.91), (3.92) e (3.93) implicam

$$
\left\|\int_{t_{0}}^{s} D G_{k}\left(x_{k}(\tau), t\right)-\int_{t_{0}}^{s} D G_{0}(x(\tau), t)\right\| \leq
$$




$$
\begin{aligned}
\leq 2 \eta\left(h_{k}(s)\right. & \left.-h_{k}(\alpha)+h_{0}(s)-h_{0}(\alpha)\right)+\eta \sum_{m=1}^{p}\left(h_{k}\left(\alpha_{m}\right)-h_{k}\left(\alpha_{m-1}\right)\right)+ \\
& +\sum_{m=1}^{p}\left\|G_{k}\left(x\left(\alpha_{m-1}\right), \alpha_{m}\right)-G_{0}\left(x\left(\alpha_{m-1}\right), \alpha_{m}\right)\right\|+ \\
+ & \sum_{m=1}^{p}\left\|G_{k}\left(x\left(\alpha_{m-1}\right), \alpha_{m-1}\right)+G_{0}\left(x\left(\alpha_{m-1}\right), \alpha_{m-1}\right)\right\|+ \\
& +h_{k}\left(\alpha_{p}\right)-h_{k}\left(\alpha_{p-1}\right)+h_{0}\left(\alpha_{p}\right)-h_{0}\left(\alpha_{p-1}\right) \leq \\
\leq 2 \eta[1+ & \left.h_{0}\left(t_{0}+\sigma\right)-h_{0}\left(t_{0}-r\right)\right]+\eta\left[h_{k}\left(t_{0}+\sigma\right)-h_{k}\left(t_{0}-r\right)\right]+ \\
& +\sum_{m=1}^{l}\left\|G_{k}\left(x\left(\alpha_{m-1}\right), \alpha_{m}\right)-G_{0}\left(x\left(\alpha_{m-1}\right), \alpha_{m}\right)\right\|+ \\
+ & \left.\sum_{m=1}^{l}\left\|G_{k}\left(x\left(\alpha_{m-1}\right), \alpha_{m-1}\right)+G_{0}\left(x\left(\alpha_{m-1}\right), \alpha_{m-1}\right)\right\|\right]
\end{aligned}
$$

Por (3.78), para todo $\mu>0$ existe um $k_{2} \in \mathbb{N}, k_{2}>k_{1}$ tal que

$$
\begin{gathered}
\sum_{m=1}^{l}\left\|G_{k}\left(x\left(\alpha_{m-1}\right), \alpha_{m}\right)-G_{0}\left(x\left(\alpha_{m-1}\right), \alpha_{m}\right)\right\|+ \\
+\sum_{m=1}^{l}\left\|G_{k}\left(x\left(\alpha_{m-1}\right), \alpha_{m-1}\right)-G_{0}\left(x\left(\alpha_{m-1}\right), \alpha_{m-1}\right)\right\| \leq \frac{\mu}{4}
\end{gathered}
$$

para $k>k_{2}$. Daí, por (3.73), existe um $k_{3} \in \mathbb{N}, k_{3}>k_{2}$ tal que para $k \in \mathbb{N}, k>k_{3}$, temos

$$
h_{k}\left(t_{0}+\sigma\right)-h_{k}\left(t_{0}-r\right) \leq h_{0}\left(t_{0}+\sigma\right)-h_{0}\left(t_{0}-r\right)+1 \text {. }
$$

Como $\eta>0$ pode ser arbitrário, então escolhemos $\eta$ de modo que a desigualdade seguinte esteja satisfeita

$$
\eta<\frac{\mu}{20\left(1+h_{0}\left(t_{0}+\sigma\right)-h_{0}\left(t_{0}-r\right)\right)}
$$

Pela desigualdade (3.94), segue que para todo $s \in\left[t_{0}+\sigma, t_{0}-r\right]$

$$
\begin{gathered}
\left\|\int_{t_{0}-r}^{s} D G_{k}\left(x_{k}(\tau), t\right)-\int_{t-0-r}^{s} D G_{0}(x(\tau), t)\right\| \leq \\
\quad \leq \frac{\mu}{4}+\frac{5 \mu\left[1+h_{0}\left(t_{0}+\sigma\right)-h_{0}\left(t_{0}-r\right)\right]}{20\left[1+h_{0}\left(t_{0}+\sigma\right)-h_{0}\left(t_{0}-r\right)\right]}=\frac{\mu}{2} .
\end{gathered}
$$


Portanto, por (3.86), temos

$$
\left\|x_{k}(s)-x(s)\right\| \leq\left\|y_{k}-x\left(t_{0}\right)\right\|+\frac{\mu}{2}
$$

para todo $s \in\left[t_{0}-r, t_{0}+\sigma\right]$ e, finalmente, tomamos $k_{*} \in \mathbb{N}$ tal que $k_{*}>k_{3}$ e $\left\|y_{k}-x\left(t_{0}\right)\right\| \leq$ $\frac{\mu}{2}$ para $k>k_{*}$. Então obtemos

$$
\left\|x_{k}(s)-x(s)\right\|<\mu
$$

para quaisquer $s \in\left[t_{0}-r, t_{0}+\sigma\right]$ e $k>k_{*}$ e o teorema está provado. 


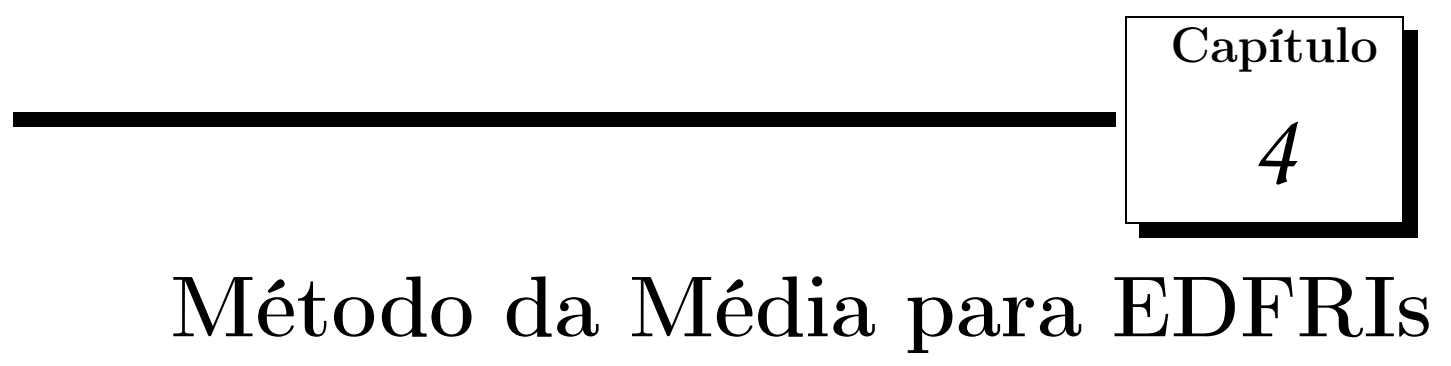

Neste capítulo, apresentaremos quatro resultados principais, a saber, versões do Princípio da Média para EDOGs, EDIs, EDFRs e EDFRIs.

O primeiro resultado principal é uma generalização do Princípio da Média para EDOGs para funções a valores em $G^{-}([-r, \infty)$ ) (compare com [26], Teorema 8.12). Tal resultado, junto com o último teorema sobre dependência contínua de soluções para EDOGs apresentado no Capítulo 3, será importante na demonstração do Princípio da Média para EDFRIs (Teorema 4.10).

Devido ao Princípio da Média para EDOGs, obtivemos, de maneira natural, uma versão do Princípio da Média para EDIs (Teorema 4.2). Tal resultado generaliza os resultados existentes por tratar de condições sobre a integral indefinida da função ao lado direito da equação e, portanto, por permitir que esta função tenha muitas descontinuidades, por exemplo.

Em seguida, trabalhamos na tradução dos resultados de M. Lakrib (veja [19]) para o caso da Análise Clássica. Como comentamos na introdução deste trabalho, Lakrib usou Análise Não Standard em seu artigo. E, neste artigo, havia um resultado que nos interessava muito se pudéssemos tê-lo através da Análise Clássica. O que fizemos, portanto, foi trabalhar os resultados de [19] a fim de obtermos os resultados correspondentes usando as ferramentas da Análise usual. Em particular, obtivemos nosso Lema 4.6, que é o resultado que nos interessava. Com isto, seguiu desta investigação, uma versão do Princípio da Média para EDFRs (Teorema 4.8). Tal versão generaliza os resultados da Análise Não Standard (veja [19], [20] e [25]), pois não precisa da hipótese de continuidade na variável tempo da função ao lado direito da equação, e também generaliza resultados da Análise Clássica (veja [15], por exemplo, que usa as hipóteses adicionais da solução da 
equação média ser limitada e da função ao lado direito da equação possuir solução quase periódica).

Finalmente, em vista do Princípio da Média para EDOGs, do nosso Lema 4.6, e da correspondência entre EDFRIs e EDOGs, obtivemos uma versão do Princípio da Média para EDFRIs que apresentaremos no Teorema 4.10.

No que segue, vamos considerar $t_{0}=0$. Assim, por exemplo, vamos escrever $[-r, \infty)$ em vez de $\left[t_{0}-r, \infty\right), G^{-}([-r, \infty))$ em vez de $G^{-}\left(\left[t_{0}-r, \infty\right)\right)$, etc.

\subsection{O Princípio da Média para EDOGs}

Vamos considerar a seguinte equação diferencial generalizada

$$
\frac{d x}{d \tau}=D G(x, t)
$$

onde $G \in \mathcal{F}(\Omega, h)$ é dada por (3.18), $\Omega=P C_{1} \times[-r, \infty)$ e $h=h_{1}+h_{2}:[-r, \infty) \rightarrow \mathbb{R}^{n}$, onde $h_{1}$ é dada por (3.5) e $h_{2}$ é dada por (3.12).

Iremos assumir que $G(x, 0)=0$, para todo $x \in P C_{1}$.

Antes de enunciamos nosso primeiro resultado, gostaríamos de observar que se uma função qualquer $H_{0} \in \mathcal{F}(\Omega, h)$ for tal que $(x, t) \mapsto H_{0}(x, t)=G_{0}(x) t$, para todo par $(x, t) \in \Omega=P C_{1} \times[-r, \infty)$, então a equação diferencial generalizada

$$
\frac{d x}{d \tau}=D H_{0}(x, t)=D\left[G_{0}(x) t\right]
$$

pode ser reescrita como uma EDO autônoma (claramente a valores no espaço abstrato $\left.G^{-}([-r, \infty))\right)$

$$
\dot{x}=G_{0}(x),
$$

pois para toda partição suficientemente fina, $\left(\tau_{i},\left[s_{i-1}, s_{i}\right]\right)$, de um subintervalo $[\alpha, \beta]$ qualquer de $[-r, \infty)$, temos

$$
\begin{aligned}
\int_{\alpha}^{\beta} D H_{0}(x(\tau), t) & \approx \sum_{i}\left[H_{0}\left(x\left(\tau_{i}\right), s_{i}\right)-H_{0}\left(x\left(\tau_{i}\right), s_{i-1}\right)\right]= \\
& =\sum_{i} G_{0}\left(x\left(\tau_{i}\right)\right)\left(s_{i}-s_{i-1}\right) \approx \int_{\alpha}^{\beta} G_{0}(x(t)) d t .
\end{aligned}
$$

O teorema que segue é o Princípio da Média para EDOGs.

Teorema 4.1. Seja $\Omega=P C_{1} \times[-r, \infty)$ e suponhamos que $G: \Omega \rightarrow G^{-}([-r, \infty))$ seja 
dada por (3.18). Consideremos a equação (4.1) e suponhamos que

$$
\limsup _{T \rightarrow \infty} \frac{h(T+\alpha)-h(\alpha)}{T} \leq C, \quad \text { onde } C=\text { constante }
$$

para todo $\alpha \geq 0$, e

$$
\lim _{T \rightarrow \infty} \frac{G(x, T)}{T}=G_{0}(x),
$$

para todo $x \in P C_{1}$. Seja $y:[-r, \infty) \rightarrow P C_{1}$ a solução única da equação diferencial ordinária autônoma

$$
\dot{y}=G_{0}(y)
$$

solução esta que pertence a $P C_{1}$ junto com sua $\rho$-vizinhança, para algum $\rho>0$, isto é, existe $\rho>0$ tal que $\left\{x \in P C_{1} ;\|z-y(t)\|<\rho\right\} \subset P C_{1}$, para todo $t \in[-r, \infty)$. Então para quaisquer $\mu>0$ e $L>0$, existe um $\varepsilon_{0}>0$ tal que para $\varepsilon \in\left(0, \varepsilon_{0}\right)$, vale a desigualdade

$$
\left\|x_{\varepsilon}(t)-\xi_{\varepsilon}(t)\right\|<\mu
$$

para $t \in\left[0, \frac{L}{\varepsilon}\right]$, onde $x_{\varepsilon}$ é uma solução da equação diferencial generalizada

$$
\frac{d x}{d \tau}=D[\varepsilon G(x, t)]
$$

em $\left[0, \frac{L}{\varepsilon}\right]$ tal que $x_{\varepsilon}(0)=y(0)$, e $\xi_{\varepsilon}$ é uma solução da equação diferencial ordinária autônoma

$$
\dot{x}=\varepsilon G_{0}(x)
$$

em $\left[0, \frac{L}{\varepsilon}\right]$ satisfazendo $\xi_{\varepsilon}(0)=y(0)$.

Prova: Para $y \in P C_{1}, t \in[-r,+\infty)$ e $\varepsilon>0$, definamos

$$
H_{\varepsilon}(y, t)=\varepsilon G\left(y, \frac{t}{\varepsilon}\right)
$$

e tomemos $h_{\varepsilon}(t)=\varepsilon h\left(\frac{t}{\varepsilon}\right)$, para $t \in[-r, \infty)$. A função $h_{\varepsilon}$ é evidentemente não decrescente e contínua à esquerda em $[-r,+\infty)$. E como $G \in \mathcal{F}(\Omega, h)$, segue da definição de $\mathcal{F}(\Omega, h)$ as seguintes desigualdades:

$$
\begin{aligned}
\left\|H_{\varepsilon}\left(y, t_{2}\right)-H_{\varepsilon}\left(y, t_{1}\right)\right\| & =\left\|\varepsilon G\left(y, \frac{t_{2}}{\varepsilon}\right)-\varepsilon G\left(y, \frac{t_{1}}{\varepsilon}\right)\right\| \leq \\
& \leq \varepsilon\left|h\left(\frac{t_{2}}{\varepsilon}\right)-h\left(\frac{t_{1}}{\varepsilon}\right)\right|=\left|h_{\varepsilon}\left(t_{2}\right)-h_{\varepsilon}\left(t_{1}\right)\right|
\end{aligned}
$$


e, similarmente,

$$
\begin{gathered}
\left\|H_{\varepsilon}\left(x, t_{2}\right)-H_{\varepsilon}\left(x, t_{1}\right)-H_{\varepsilon}\left(y, t_{2}\right)+H_{\varepsilon}\left(y, t_{1}\right)\right\|= \\
=\left\|\varepsilon G\left(x, \frac{t_{2}}{\varepsilon}\right)-\varepsilon G\left(x, \frac{t_{1}}{\varepsilon}\right)-\varepsilon G\left(y, \frac{t_{2}}{\varepsilon}\right)+\varepsilon G\left(y, \frac{t_{1}}{\varepsilon}\right)\right\| \leq \\
\leq\|x-y\| \varepsilon\left|h\left(\frac{t_{2}}{\varepsilon}\right)-h\left(\frac{t_{1}}{\varepsilon}\right)\right|=\|x-y\|\left|h_{\varepsilon}\left(t_{2}\right)-h_{\varepsilon}\left(t_{1}\right)\right|,
\end{gathered}
$$

para quaisquer $x, y \in P C_{1}$ e quaisquer $t_{1}, t_{2} \in[-r,+\infty)$. Logo $H_{\varepsilon} \in \mathcal{F}\left(\Omega, h_{\varepsilon}\right)$, para $\varepsilon>0$.

Agora, consideremos $y \in P C_{1}$. Então

$$
\lim _{T \rightarrow \infty} \frac{G(y, T)-G(y, 0)}{T}=\lim _{T \rightarrow \infty} \frac{G(y, T)}{T}=G_{0}(y)
$$

e, portanto, (4.3) e (4.2) implicam que, para todo $\eta>0$, existe $R>0$ tal que para $T>R$, temos

$$
\begin{aligned}
\left\|G_{0}(y)\right\| & \leq\left\|G_{0}(y)-\frac{G(y, T)-G(y, 0)}{T}\right\|+\frac{\|G(y, T)-G(y, 0)\|}{T} \leq \\
& \leq \eta+\frac{h(T)-h(0)}{T}<2 \eta+C,
\end{aligned}
$$

pois $G \in \mathcal{F}(\Omega, h)$ implica que $\|G(y, T)-G(y, 0)\| \leq h(T)-h(0)$. E como $\eta>0$ pode ser escolhido arbitrariamente pequeno, temos

$$
\left\|G_{0}(y)\right\| \leq C, \quad y \in P C_{1}
$$

Analogamente, se $x, y \in P C_{1}$, então para todo $\eta>0$, existirá um $R>0$ tal que para todo $T>R$, teremos

$$
\begin{gathered}
\left\|G_{0}(x)-G_{0}(y)\right\|<\eta+\frac{\|G(y, T)-G(y, 0)-G(x, T)+G(x, 0)\|}{T} \leq \\
\leq \eta+\|x-y\| \frac{h(T)-h(0)}{T} \leq \eta+(\eta+C)\|y-x\| \leq \eta(1+\|y-x\|)+C\|y-x\|,
\end{gathered}
$$

e, novamente, como $\eta>0$ pode ser escolhido suficientemente pequeno, obtemos

$$
\left\|G_{0}(x)-G_{0}(y)\right\| \leq C\|y-x\|, \quad x, y \in P C_{1}
$$

Para $y \in P C_{1}$ e $t \in[-r, \infty), t \neq 0$, temos

$$
\lim _{\varepsilon \rightarrow 0^{+}} H_{\varepsilon}(y, t)=\lim _{\varepsilon \rightarrow 0^{+}} \varepsilon G\left(y, \frac{t}{\varepsilon}\right)=\lim _{\varepsilon \rightarrow 0^{+}} t \frac{\varepsilon}{t} G\left(y, \frac{t}{\varepsilon}\right)=t G_{0}(y)
$$


e, para $t=0$, temos

$$
\lim _{\varepsilon \rightarrow 0^{+}} H_{\varepsilon}(y, 0)=\lim _{\varepsilon \rightarrow 0^{+}} \varepsilon G\left(y, \frac{0}{\varepsilon}\right)=0 .
$$

Seja $H_{0}(y, t)=t G_{0}(y)$, para $y \in P C_{1}$ e $t \geq-r$. Então, pelas relações dadas acima, temos

$$
\lim _{\varepsilon \rightarrow 0^{+}} H_{\varepsilon}(y, t)=H_{0}(y, t) .
$$

Por (4.8) e (4.6), $H_{0} \in \mathcal{F}(\Omega, h)$, onde $h_{0}(t)=C t, t \geq-r$. Além disso, para $-r \leq t_{1}<$ $t_{2}<+\infty$, obtemos pela definição

$$
\begin{aligned}
h_{\varepsilon}\left(t_{2}\right)-h_{\varepsilon}\left(t_{1}\right) & =\varepsilon\left[h\left(\frac{t_{2}}{\varepsilon}\right)-h\left(\frac{t_{1}}{\varepsilon}\right)\right]= \\
& =\left(t_{2}-t_{1}\right) \frac{\varepsilon}{t_{2}-t_{1}}\left[h\left(\frac{t_{2}-t_{1}}{\varepsilon}+\frac{t_{1}}{\varepsilon}\right)-h\left(\frac{t_{1}}{\varepsilon}\right)\right]
\end{aligned}
$$

e daí, pela hipótese, obtemos

$$
\limsup _{\varepsilon \rightarrow 0^{+}}\left[h_{\varepsilon}\left(t_{2}\right)-h_{\varepsilon}\left(t_{1}\right)\right] \leq C\left(t_{2}-t_{1}\right)=h_{0}\left(t_{2}\right)-h_{0}\left(t_{1}\right)
$$

já que temos

$$
\lim _{\varepsilon \rightarrow 0^{+}} \frac{t_{2}-t_{1}}{\varepsilon}=+\infty
$$

Podemos notar facilmente que (4.10) também está satisfeita no caso em que $t_{1}=t_{2}$.

Usando o fato que $y:[-r, \infty) \rightarrow P C_{1}$ é uma solução de (4.4) e usando as propriedades da integral generalizada de Perron como mencionamos no parágrafo anterior ao enunciado do teorema, temos

$$
y\left(s_{2}\right)-y\left(s_{1}\right)=\int_{s_{1}}^{s_{2}} G_{0}(y(\tau)) d \tau=\int_{s_{1}}^{s_{2}} D\left[G_{0}(y(\tau)) t\right]=\int_{s_{1}}^{s_{2}} D H_{0}(y(\tau), t)
$$

para quaisquer $s_{1}, s_{2} \in[-r,+\infty)$. Portanto, $y$ é uma solução da equação diferencial ordinária generalizada

$$
\frac{d y}{d \tau}=D H_{0}(y, t)
$$

em $[0,+\infty)$ e, pelas condições do teorema, esta solução é unicamente determinada.

Desta forma, mostramos que todas as hipóteses do Teorema 3.6 estão satisfeitas para o caso do parâmetro contínuo $\varepsilon \rightarrow 0^{+}$. Usando o resultado do Teorema 3.6, para todo $\mu>0$ e todo $L>0$, existe um valor $\varepsilon_{0}>0$ tal que para $\varepsilon \in\left(0, \varepsilon_{0}\right)$ e existe uma solução $y_{\varepsilon}$ da equação diferencial generalizada 


$$
\frac{d y}{d \tau}=D H_{\varepsilon}(y, t)
$$

no intervalo $[0, L]$ satisfazendo $y_{\varepsilon}(0)=y(0)$ e

$$
\left\|y_{\varepsilon}(s)-y(s)\right\| \leq \mu
$$

para todo $s \in[0, L]$, onde $y$ é solução de (4.4).

Para a solução $y_{\varepsilon}:[0, L] \rightarrow P C_{1}$ de (4.11), também temos

$$
y_{\varepsilon}\left(s_{2}\right)-y_{\varepsilon}\left(s_{1}\right)=\int_{s_{1}}^{s_{2}} D H_{\varepsilon}\left(y_{\varepsilon}(\tau), t\right)=\varepsilon \int_{s_{1}}^{s_{2}} D G\left(y_{\varepsilon}(\tau), \frac{t}{\varepsilon}\right)
$$

sempre que $s_{1}, s_{2} \in[0, L]$.

Para $t \in\left[0, \frac{L}{\varepsilon}\right]$, denotemos $x_{\varepsilon}(t)=y_{\varepsilon}(\varepsilon t)$. Então vale

$$
\begin{aligned}
x_{\varepsilon}\left(t_{2}\right)-x_{\varepsilon}\left(t_{1}\right) & =y_{\varepsilon}\left(\varepsilon t_{2}\right)-y_{\varepsilon}\left(\varepsilon t_{1}\right)=\varepsilon \int_{\varepsilon t_{1}}^{\varepsilon t_{2}} D G\left(y_{\varepsilon}(\sigma), \frac{s}{\varepsilon}\right)= \\
& =\varepsilon \int_{\varepsilon t_{1}}^{\varepsilon t_{2}} D G\left(x_{\varepsilon}\left(\frac{\sigma}{\varepsilon}\right), \frac{s}{\varepsilon}\right)
\end{aligned}
$$

para $t_{2}, t_{1} \in\left[0, \frac{L}{\varepsilon}\right]$. Aplicando o teorema de mudança de variável (Teorema 1.11) para $\varphi(\sigma)=\frac{\sigma}{\varepsilon}$, obtemos

$$
\int_{\varepsilon t_{1}}^{\varepsilon t_{2}} D G\left(x_{\varepsilon}\left(\frac{\sigma}{\varepsilon}\right), \frac{s}{\varepsilon}\right)=\int_{\varphi\left(\varepsilon t_{1}\right)}^{\varphi\left(\varepsilon t_{2}\right)} D G\left(x_{\varepsilon}(\tau), t\right)=\int_{t_{1}}^{t_{2}} D G\left(x_{\varepsilon}(\tau), t\right)
$$

para quaisquer $t_{1}, t_{2} \in\left[0, \frac{L}{\varepsilon}\right]$. Isto, junto com a igualdade anterior, implica que

$$
x_{\varepsilon}\left(t_{2}\right)-x_{\varepsilon}\left(t_{1}\right)=\varepsilon \int_{t_{1}}^{t_{2}} D G\left(x_{\varepsilon}(\tau), t\right)
$$

para $t_{2}, t_{1} \in\left[0, \frac{L}{\varepsilon}\right] \mathrm{e}$

$$
x_{\varepsilon}(0)=y_{\varepsilon}(0)=y(0) \text {. }
$$

Portanto, a função $x_{\varepsilon}:\left[0, \frac{L}{\varepsilon}\right] \rightarrow P C_{1}$ é uma solução da equação diferencial generalizada (4.5) em $\left[0, \frac{L}{\varepsilon}\right]$.

Analogamente, podemos mostrar que a função $\xi_{\varepsilon}:\left[0, \frac{L}{\varepsilon}\right] \rightarrow P C_{1}$ dada por $\xi_{\varepsilon}(t)=y(\varepsilon t)$ é uma solução da equação diferencial ordinária autônoma (4.6) em $\left[0, \frac{L}{\varepsilon}\right]$.

Finalmente, por (4.12), obtemos 


$$
\left\|x_{\varepsilon}(t)-\xi_{\varepsilon}(t)\right\|=\left\|y_{\varepsilon}(\varepsilon t)-y(\varepsilon t)\right\|<\mu
$$

para todo $t \in\left[0, \frac{L}{\varepsilon}\right]$ e o teorema está provado.

\subsection{O Princípio da Média para EDIs}

Uma aplicação que segue imediatamente do Teorema 4.1 é o seguinte Princípio da Média para EDIs, que generaliza o resultado e Š. Schwabik encontrado em [26], Teorema 8.14. Quando os operadores de impulsos forem o operador identidade, estaremos no caso particular das EDOs clássicas e o resultado desta seção generaliza os existentes.

Teorema 4.2. Seja $\Omega=B \times[0, \infty)$, onde $B=\left\{x \in \mathbb{R}^{n}:|x|<c\right\}, c>0$. Suponhamos que $f: \Omega \rightarrow \mathbb{R}^{n}$ satisfaça as condições $(A)$ e $(B)$. Suponhamos que uma sequência de pontos $0 \leq t_{1}<t_{2}<\ldots$ dada seja tal que

$$
\limsup _{r \rightarrow \infty} \sum_{\alpha \leq t_{i} \leq \alpha+r} 1 \leq d
$$

para todo $\alpha \geq 0$ e suponhamos que $I_{i}: B \rightarrow \mathbb{R}^{n}, i=0,1,2, \ldots$, seja uma sequência de operadores de impulso que satisfazem as condições $\left(A^{\prime}\right)$ e $\left(B^{\prime}\right)$. Suponhamos, ainda, que

$$
\begin{gathered}
\lim _{T \rightarrow \infty} \frac{1}{T} \int_{0}^{T} f(y, s) d s=f_{0}(y), \quad x \in B, \\
\lim _{T \rightarrow \infty} \frac{1}{T} \sum_{0 \leq t_{i}<T} I_{i}(x)=I_{0}(x), \quad x \in B \quad e \\
\lim _{T \rightarrow \infty} \frac{1}{T} \int_{\alpha}^{T+\alpha}[M(s)+L(s)] d s \leq c, \quad c=\text { constante },
\end{gathered}
$$

para todo $t \in[0,+\infty)$ e $\alpha \geq 0$. Seja $y \in G^{-}([0, \infty))$ a solução unicamente determinada da equação diferencial autônoma

$$
\dot{y}=f_{0}(y)+I_{0}(y)
$$

Então para todo $\mu>0$ e todo $L>0$, existe um $\varepsilon_{0}>0$ tal que para $\varepsilon \in\left(0, \varepsilon_{0}\right)$, a desigualdade

$$
\left|y^{\varepsilon}(t)-\xi^{\varepsilon}(t)\right|<\mu
$$

vale em $\left[0, \frac{L}{\varepsilon}\right]$, onde $y^{\varepsilon}$ é a solução da EDI 


$$
\left\{\begin{array}{l}
\dot{y}=\varepsilon f(y, t), t \neq t_{i} \\
\Delta y\left(t_{i}\right)=y\left(t_{i}+\right)-y\left(t_{i}\right)=\varepsilon I_{i}\left(y\left(t_{i}\right)\right), \quad i=1,2, \ldots
\end{array}\right.
$$

em $\left[0, \frac{L}{\varepsilon}\right]$ tal que $y^{\varepsilon}(0)=y(0)$, e $\xi^{\varepsilon}$ é a solução do sistema médio

$$
\dot{y}=\varepsilon\left[f_{0}(y)+I_{0}(y)\right]
$$

em $\left[0, \frac{L}{\varepsilon}\right]$ tal que $\xi^{\varepsilon}(0)=y(0)$.

Prova: Seja

$$
G(y, t)=\int_{0}^{t} f(y, s) d s+\sum_{i=1}^{\infty} H_{t_{i}}(t) I_{i}(y) .
$$

Não é difícil de mostrar que a EDOG

$$
\frac{d x}{d \tau}=D[\varepsilon G(x, t)]
$$

corresponde ao sistema ordinário (4.13) e $H_{\nu}(t)=0$ para $t \in[0, \nu]$ e $H_{\nu}(t)=0$ para $t>\nu$.

Pelas hipóteses, é fácil verificar que $G: \Omega \rightarrow \mathbb{R}^{n}$ pertence à classe $\mathcal{F}(\Omega, h)$, onde

$$
h(t)=\int_{0}^{t}[M(s)+L(s)] d s+\max \left(K_{1}, K_{2}\right) \sum_{i=1}^{\infty} H_{t_{i}}(t), \quad t \in[0, \infty) .
$$

Além disso, as hipóteses também implicam que

$$
\lim _{T \rightarrow \infty} \frac{G(y, T)}{T}=f_{0}(y)+I_{0}(y)=G_{0}(y)
$$

e

$$
\limsup _{T \rightarrow \infty} \frac{h(T+\alpha)-h(\alpha)}{T} \leq c+\max \left(K_{1}, K_{2}\right) d
$$

para $x \in B$ e $\alpha \geq 0$.

Assim, as hipóteses do Teorema 4.1 estão satisfeitas e o resultado segue imediatamente da correspondência entre o sistema impulsivo (4.13) e o sistema generalizado (4.15) e da correspondência entre o sistema médio ordinário (4.14) e $\dot{x}=G_{0}(x)$.

\subsection{O Princípio da Média para EDFRs}

Vamos dar continuidade ao nosso trabalho apresentando um Princípio da Média para EDFRs. Usaremos várias idéias de [19]. Mas em [19], usa-se a teoria de Análise Não 
Standard.

No que segue, procuramos demonstrar resultados análogos aos de [19] através da Análise Clássica.

Consideremos a EDFR

$$
\left\{\begin{array}{l}
\dot{y}=f\left(y_{t}, \frac{t}{\varepsilon}\right) \\
y_{0}=\phi
\end{array}\right.
$$

onde $\phi \in G^{-}\left([-r, 0], \mathbb{R}^{n}\right)$ e $\varepsilon>0$ é um parâmetro pequeno.

Suponhamos que exista o limite

$$
\lim _{T \rightarrow \infty} \frac{1}{T} \int_{0}^{T} f(\psi, s) d s=f_{0}(\psi)
$$

para cada $\psi \in G^{-}\left([-r, 0], \mathbb{R}^{n}\right)$, onde $f$ satisfaz a seguinte condição $\left(\mathcal{B}^{*}\right)$ (que implica na condição $\left(B^{*}\right)$ apresentada anteriormente):

$\left(\mathcal{B}^{*}\right)$ existe uma função localmente Lebesgue integrável $L:[0, \infty) \rightarrow \mathbb{R}$ tal que para $\psi, \varphi \in G^{-}\left([-r, 0], \mathbb{R}^{n}\right)$ e $u_{1}, u_{2} \in[0,+\infty)$,

$$
\left|\int_{u_{1}}^{u_{2}}[f(\psi, s)-f(\varphi, s)] d s\right| \leq \int_{u_{1}}^{u_{2}} L(s)\|\psi-\varphi\| d s .
$$

Consideremos, também, a seguinte condição adicional:

$\left(\mathcal{C}^{*}\right)$ existem constantes $C, N>0$ tais que para todo $u \in[0,+\infty)$,

$$
\int_{u-r}^{u} L(s) d s<N \quad \text { e } \quad \lim _{T \rightarrow \infty} \frac{1}{T} \int_{0}^{T} L(\sigma)=C .
$$

Note que, pela continuidade da integral indefinida de $f$, que segue pelo Lema de SaksHenstock (Lema 1.8), então, para qualquer $\varepsilon>0$ suficientemente pequeno, também vale

$$
\int_{u-r}^{u+\varepsilon} L(s) d s<N, \quad u \in[0,+\infty)
$$

já que $\int_{u-r}^{u+\varepsilon} L(s) d s=\int_{u-r}^{u} L(s) d s+\int_{u}^{u+\varepsilon} L(s) d s$ e esta última integral pode ser tomada tão pequena quanto se queira.

Note, também, que se $f$ satisfizer as condições $\left(\mathcal{B}^{*}\right)$ e $\left(\mathcal{C}^{*}\right)$, então, para todo $y \in P C_{1}$, 


$$
\begin{aligned}
\left|f_{0}\left(y_{s}\right)-f_{0}\left(y_{t}\right)\right| & \leq \lim _{T \rightarrow \infty} \frac{1}{T}\left|\int_{0}^{T}\left[f\left(y_{s}, \sigma\right)-f\left(y_{t}, \sigma\right)\right] d \sigma\right| \leq \\
& \leq \lim _{T \rightarrow \infty} \frac{1}{T} \int_{0}^{T} L(\sigma)\left\|y_{s}-y_{t}\right\| d \sigma<C\left\|y_{s}-y_{t}\right\| .
\end{aligned}
$$

Isto vai implicar que, para $\theta \in[-r, 0]$, se $y$ for solução de $(4.33)$ e $s, t \in[0, \infty)$, com $s \leq t$, então teremos

$$
\begin{aligned}
|y(s+\theta)-y(t+\theta)| & =\left|\int_{s+\theta}^{t+\theta} f_{0}\left(y_{\sigma}\right) d \sigma\right| \leq \\
& \leq \int_{s+\theta}^{t+\theta}\left|f_{0}\left(y_{\sigma}\right)-f_{0}(0)\right| d \sigma+\int_{s+\theta}^{t+\theta}\left|f_{0}(0)\right| d \sigma \leq \\
& \leq C \int_{s+\theta}^{t+\theta}|| y_{\sigma} \| d \sigma+(t-s)\left|f_{0}(0)\right| .
\end{aligned}
$$

Portanto,

$$
\begin{aligned}
\left\|y_{s}-y_{t}\right\| & =\sup _{\theta \in[-r, 0]}|y(s+\theta)-y(t+\theta)| \leq \\
& \leq C(t-s) \sup _{\sigma \in[s-r, t]}\left\|y_{\sigma}\right\|+(t-s)\left|f_{0}(0)\right| .
\end{aligned}
$$

Daí, fazendo $t-s \rightarrow 0$, temos $\left\|y_{s}-y_{t}\right\| \rightarrow 0$, o que quer dizer que $y_{t}$ como função de $t$, para $t$ variando em $[0, \infty)$, é uma função contínua.

Consideremos a seguinte EDFR média

$$
\left\{\begin{array}{l}
\dot{y}=f_{0}\left(y_{t}\right) \\
y_{0}=\phi
\end{array}\right.
$$

onde $f_{0}$ é dada por (4.17).

A seguir, vamos provar vários resultados auxiliares para, finalmente apresentarmos uma versão do Princípio da Média para EDFRs.

Lema 4.3. Consideremos (4.17). Então, para todo $t>0$ e todo $S>0$, vale

$$
\lim _{\varepsilon \rightarrow 0+} \frac{1}{S} \int_{t / \varepsilon}^{t / \varepsilon+S} f(\psi, s) d s=f_{0}(\psi), \quad \psi \in G^{-}\left([-r, 0], \mathbb{R}^{n}\right)
$$

Prova: Seja $\varepsilon>0$ dado arbitrariamente. Então, por (4.17), existe $N_{\varepsilon}>0$, digamos $N_{\varepsilon}>\frac{1}{\sqrt{\varepsilon}}$, tal que para todo $T>N_{\varepsilon}$, vale

$$
\left|f_{0}(\psi)-\frac{1}{T} \int_{0}^{T} f(\psi, s) d s\right|<\frac{\varepsilon^{2}}{2} .
$$


Seja $t>0$ tal que $t>\varepsilon N_{\varepsilon}>\varepsilon \frac{1}{\sqrt{\varepsilon}}=\sqrt{\varepsilon}$. Então $\frac{t}{\varepsilon}>N_{\varepsilon}$ e, portanto, $\frac{t}{\varepsilon}+S>N_{\varepsilon}$, para $S>0$. Logo, por (4.21), temos

$$
\begin{gathered}
\left|f_{0}(\psi)-\frac{1}{t / \varepsilon+S} \int_{0}^{t / \varepsilon+S} f(\psi, s) d s\right|<\frac{\varepsilon^{2}}{2} \quad \mathrm{e} \\
\left|f_{0}(\psi)-\frac{1}{t / \varepsilon} \int_{0}^{t / \varepsilon} f(\psi, s) d s\right|<\frac{\varepsilon^{2}}{2}
\end{gathered}
$$

e, portanto,

$$
\left|\frac{1}{t / \varepsilon+S} \int_{0}^{t / \varepsilon+S} f(\psi, s) d s-\frac{1}{t / \varepsilon} \int_{0}^{t / \varepsilon} f(\psi, s) d s\right|<\varepsilon^{2} .
$$

Por outro lado,

$$
\begin{gathered}
\frac{1}{S} \int_{t / \varepsilon}^{t / \varepsilon+S} f(\psi, s) d s=\frac{1}{S} \int_{0}^{t / \varepsilon+S} f(\psi, s) d s-\frac{1}{S} \int_{0}^{t / \varepsilon} f(\psi, s) d s= \\
=\left(\frac{t / \varepsilon+S}{t / \varepsilon+S}\right) \frac{1}{S} \int_{0}^{t / \varepsilon+S} f(\psi, s) d s-\left(\frac{t / \varepsilon}{t / \varepsilon}\right) \frac{1}{S} \int_{0}^{t / \varepsilon} f(\psi, s) d s= \\
=\frac{1}{t / \varepsilon+S}\left(\frac{t}{\varepsilon S}+1\right) \int_{0}^{t / \varepsilon+S} f(\psi, s) d s-\frac{t}{\varepsilon S} \cdot \frac{1}{t / \varepsilon} \int_{0}^{t / \varepsilon} f(\psi, s) d s= \\
=\frac{1}{t / \varepsilon+S} \int_{0}^{t / \varepsilon+S} f(\psi, s) d s+\frac{t}{\varepsilon S}\left[\frac{1}{t / \varepsilon+S} \int_{0}^{t / \varepsilon+S} f(\psi, s) d s-\frac{1}{t / \varepsilon} \int_{0}^{t / \varepsilon} f(\psi, s) d s\right] .
\end{gathered}
$$

Logo

$$
\begin{gathered}
\left|\frac{1}{t / \varepsilon+S} \int_{t / \varepsilon}^{t / \varepsilon+S} f(\psi, s) d s-f_{0}(\psi)\right| \leq\left|\frac{1}{t / \varepsilon+S} \int_{0}^{t / \varepsilon+S} f(\psi, s) d s-f_{0}(\psi)\right|+ \\
+\frac{t}{\varepsilon S}\left|\frac{1}{t / \varepsilon+S} \int_{0}^{t / \varepsilon+S} f(\psi, s) d s-\frac{1}{t / \varepsilon} \int_{0}^{t / \varepsilon} f(\psi, s) d s\right|<\frac{\varepsilon}{2}+\frac{t}{\varepsilon S} \cdot \varepsilon^{2}
\end{gathered}
$$

que tende a zero quando $\varepsilon \rightarrow 0^{+}$e o resultado está provado.

A demonstração do lema abaixo é análoga à demonstração acima.

Lema 4.4. Consideremos (4.17). Então, para todo $t>0$ e todo $\alpha>0$, vale

$$
\lim _{\varepsilon \rightarrow 0+} \frac{1}{\alpha / \varepsilon} \int_{t / \varepsilon}^{t / \varepsilon+\alpha / \varepsilon} f(\psi, s) d s=f_{0}(\psi), \quad \psi \in G^{-}\left([-r, 0], \mathbb{R}^{n}\right) .
$$

Prova: Seja $\varepsilon>0$ dado arbitrariamente. Então, por (4.17), existe $N_{\varepsilon}>0$, digamos 
$N_{\varepsilon}>\frac{1}{\sqrt{\varepsilon}}$, tal que para todo $T>N_{\varepsilon}$, vale

$$
\left|f_{0}(\psi)-\frac{1}{T} \int_{0}^{T} f(\psi, s) d s\right|<\frac{\varepsilon^{2}}{2}
$$

Seja $t>0$ tal que $t>\varepsilon N_{\varepsilon}>\varepsilon \frac{1}{\sqrt{\varepsilon}}=\sqrt{\varepsilon}$. Então $\frac{t}{\varepsilon}>N_{\varepsilon}$ e, portanto, $\frac{t}{\varepsilon}+\frac{\alpha}{\varepsilon}>N_{\varepsilon}$, para $\alpha>0$. Logo, por (4.22), valem as desigualdades abaixo

$$
\begin{gathered}
\left|f_{0}(\psi)-\frac{1}{t / \varepsilon+\alpha / \varepsilon} \int_{0}^{t / \varepsilon+\alpha / \varepsilon} f(\psi, s) d s\right|<\frac{\varepsilon^{2}}{2} \quad \mathrm{e} \\
\left|f_{0}(\psi)-\frac{1}{t / \varepsilon} \int_{0}^{t / \varepsilon} f(\psi, s) d s\right|<\frac{\varepsilon^{2}}{2} .
\end{gathered}
$$

Portanto,

$$
\left|\frac{1}{t / \varepsilon+\alpha / \varepsilon} \int_{0}^{t / \varepsilon+\alpha / \varepsilon} f(\psi, s) d s-\frac{1}{t / \varepsilon} \int_{0}^{t / \varepsilon} f(\psi, s) d s\right|<\varepsilon^{2} .
$$

Por outro lado,

$$
\begin{gathered}
\frac{\varepsilon}{\alpha} \int_{t / \varepsilon}^{t / \varepsilon+\alpha / \varepsilon} f(\psi, s) d s=\frac{1}{\alpha / \varepsilon} \int_{0}^{t / \varepsilon+\alpha / \varepsilon} f(\psi, s) d s-\frac{1}{\alpha / \varepsilon} \int_{0}^{t / \varepsilon} f(\psi, s) d s= \\
=\left(\frac{t / \varepsilon+\alpha / \varepsilon}{t / \varepsilon+\alpha / \varepsilon}\right) \frac{1}{\alpha / \varepsilon} \int_{0}^{t / \varepsilon+\alpha / \varepsilon} f(\psi, s) d s-\left(\frac{t / \varepsilon}{t / \varepsilon}\right) \frac{1}{\alpha / \varepsilon} \int_{0}^{t / \varepsilon} f(\psi, s) d s= \\
=\frac{1}{t / \varepsilon+\alpha / \varepsilon}\left(\frac{t}{\alpha}+1\right) \int_{0}^{t / \varepsilon+\alpha / \varepsilon} f(\psi, s) d s-\frac{t}{\alpha} \cdot \frac{1}{t / \varepsilon} \int_{0}^{t / \varepsilon} f(\psi, s) d s= \\
=\frac{1}{t / \varepsilon+\alpha / \varepsilon} \int_{0}^{t / \varepsilon+\alpha / \varepsilon} f(\psi, s) d s+ \\
+\frac{t}{\alpha}\left[\frac{1}{t / \varepsilon+\alpha / \varepsilon} \int_{0}^{t / \varepsilon+\alpha / \varepsilon} f(\psi, s) d s-\frac{1}{t / \varepsilon} \int_{0}^{t / \varepsilon} f(\psi, s) d s\right] .
\end{gathered}
$$

Logo

$$
\begin{aligned}
& \left|\frac{\varepsilon}{\alpha} \int_{t / \varepsilon}^{t / \varepsilon+\alpha / \varepsilon} f(\psi, s) d s-f_{0}(\psi)\right| \leq\left|\frac{1}{t / \varepsilon+\alpha / \varepsilon} \int_{0}^{t / \varepsilon+\alpha / \varepsilon} f(\psi, s) d s-f_{0}(\psi)\right|+ \\
& \quad+\frac{t}{\alpha}\left|\frac{1}{t / \varepsilon+\alpha / \varepsilon} \int_{0}^{t / \varepsilon+\alpha / \varepsilon} f(\psi, s) d s-\frac{1}{t / \varepsilon} \int_{0}^{t / \varepsilon} f(\psi, s) d s\right|<\frac{\varepsilon}{2}+\frac{t}{\alpha} \cdot \varepsilon^{2}
\end{aligned}
$$

que tende a zero quando $\varepsilon \rightarrow 0^{+}$e o resultado está provado. 
O corolário seguinte segue do Lema 4.4.

Corolário 4.5. Sejam $t>0$ e $\alpha>0$. Então, para todo $y \in P C_{1}$, vale

$$
\lim _{\varepsilon \rightarrow 0+} \frac{\varepsilon}{\alpha} \int_{t / \varepsilon}^{t / \varepsilon+\alpha / \varepsilon} f\left(y_{t}, s\right) d s=f_{0}\left(y_{t}\right) .
$$

Prova: A demonstração segue imediatamente do lema anterior, pois o corolário é um caso particular do lema anterior, já que $y_{t} \in G^{-}\left([-r, 0], \mathbb{R}^{n}\right)$.

Agora estamos em condições de provar o resultado que nos interessa que é o Lema 4.6 abaixo. Ele será essencial para a demonstração do Princípio da Média para EDFRs nesta seção e para a demonstração do Princípio da Média para EDFRI na seção seguinte.

Lema 4.6. Seja y uma solução de (4.20) e suponhamos que $f$ satisfaça as condições $\left(\mathcal{B}^{*}\right)$ $e\left(\mathcal{C}^{*}\right)$. Então, para todo $t>0$, vale

$$
\lim _{\varepsilon \rightarrow 0+} \int_{0}^{t} f\left(y_{s}, \frac{s}{\varepsilon}\right) d s=\int_{0}^{t} f_{0}\left(y_{s}\right) d s .
$$

Prova: Sejam $\varepsilon>$ e $t>0$ fixados arbitrários.

Para $s \geq 0$ e $\psi \in G^{-}\left([-r, \infty), \mathbb{R}^{n}\right)$, definamos

$$
f_{1}(\psi, s)=f(\psi, s)-f_{0}(\psi)
$$

Seja $\delta$ um calibre correspondente a $\varepsilon>0$ na definição da integral $\int_{0}^{t} f_{1}\left(y_{\sigma}, \frac{\sigma}{\varepsilon}\right) d \sigma$ e consideremos uma partição $\left(\tau_{i},\left[s_{i}, s_{i+1}\right]\right), i=0,1,2, \ldots, m-1$ de $[0, t]$ que seja $\delta$-fina. Então,

$$
\begin{gathered}
\left|\int_{0}^{t} f\left(y_{s}, \frac{s}{\varepsilon}\right) d s-\int_{0}^{t} f_{0}\left(y_{s}\right) d s\right|=\left|\int_{0}^{t} f_{1}\left(y_{s}, \frac{s}{\varepsilon}\right) d s\right| \leq \\
\leq \sum_{i=0}^{m-1}\left|\int_{s_{i}}^{s_{i+1}}\left[f_{1}\left(y_{s}, \frac{s}{\varepsilon}\right)-f_{1}\left(y_{s_{i}}, \frac{s}{\varepsilon}\right)\right] d s\right|+\sum_{i=0}^{m-1}\left|\int_{s_{i}}^{s_{i+1}} f_{1}\left(y_{s}, \frac{s_{i}}{\varepsilon}\right) d s\right| \\
\leq \sum_{i=0}^{m-1}\left|\int_{s_{i}}^{s_{i+1}}\left[f\left(y_{s}, \frac{s}{\varepsilon}\right)-f\left(y_{s_{i}}, \frac{s}{\varepsilon}\right)\right] d s\right|+\sum_{i=0}^{m-1}\left|\int_{s_{i}}^{s_{i+1}}\left[f_{0}\left(y_{s}\right)-f_{0}\left(y_{s_{i}}\right)\right] d s\right|+ \\
+\sum_{i=0}^{m-1}\left|\int_{s_{i}}^{s_{i+1}} f_{1}\left(y_{s}, \frac{s_{i}}{\varepsilon}\right) d s\right|
\end{gathered}
$$

Como podemos supor, sem perda de generalidade, que o calibre $\delta$ é tal que $\delta(\xi)<\frac{\varepsilon}{2}$, para todo $\xi \in[0, t]$, então, usando a condição $\left(\mathcal{B}^{*}\right)$, obtemos 


$$
\sum_{i=0}^{m-1}\left|\int_{s_{i}}^{s_{i+1}}\left[f\left(y_{s}, \frac{s}{\varepsilon}\right)-f\left(y_{s_{i}}, \frac{s}{\varepsilon}\right)\right] d s\right| \leq \sum_{i=0}^{m-1} \int_{s_{i}}^{s_{i+1}} L(s)\left\|y_{s}-y_{t_{i}}\right\| d s
$$

Por (4.19), para cada $i=0,1,2, \ldots$ e cada $s \in\left[s_{i}, s_{i+1}\right]$, temos

$$
\begin{aligned}
\left\|y_{s}-y_{t_{i}}\right\| & <C\left(s-t_{i}\right) \sup _{\sigma \in\left[t_{i}-r, s\right]}\left\|y_{\sigma}\right\|+\left(s-t_{i}\right)\left|f_{0}(0)\right|< \\
& <C 2 \delta\left(\tau_{i}\right) \sup _{\sigma \in\left[t_{i}-r, t_{i+1}\right]}\left\|y_{\sigma}\right\|+2 \delta\left(\tau_{i}\right)\left|f_{0}(0)\right|< \\
& <C \varepsilon \sup _{\sigma \in\left[t_{i}-r, t_{i+1}\right]}\left\|y_{\sigma}\right\|+\varepsilon\left|f_{0}(0)\right| .
\end{aligned}
$$

Portanto,

$$
\sup _{s \in\left[t_{i}, t_{i+1}\right]}\left\|y_{s}-y_{t_{i}}\right\| \leq C \varepsilon \sup _{\sigma \in\left[t_{i}-r, t_{i+1}\right]}\left\|y_{\sigma}\right\|+\varepsilon\left|f_{0}(0)\right|
$$

que pode ser feito suficientemente pequeno pela arbitrariedade de $\varepsilon$, digamos

$$
\sup _{s \in\left[t_{i}, t_{i+1}\right]}\left\|y_{s}-y_{t_{i}}\right\| \leq \frac{\eta}{\max (N, C) 2^{i+1}}
$$

Daí, substituindo (4.25) em (4.24) e usando a condição $\left(\mathcal{C}^{*}\right)$, obtemos

$$
\begin{gathered}
\sum_{i=0}^{m-1}\left|\int_{s_{i}}^{s_{i+1}}\left[f\left(y_{s}, \frac{s}{\varepsilon}\right)-f\left(y_{s_{i}}, \frac{s}{\varepsilon}\right)\right] d s\right|< \\
<\sum_{i=0}^{m-1} \sup _{\sigma \in\left[t_{i}, t_{i+1}\right]}\left\|y_{\sigma}-y_{t_{i}}\right\| \int_{s_{i}}^{s_{i+1}} L(s) d s<\sum_{i=0}^{m-1} \frac{\eta}{\max (N, C) 2^{i+1}} N<\eta
\end{gathered}
$$

e $\eta$ pode ser feito tão pequeno quanto se queira (pela arbitrariedade de $\varepsilon$ ). Logo o primeiro somando ao lado direito de (4.23) tende a zero quando $\varepsilon \rightarrow 0$.

Agora, usando (4.18) e (4.25), para cada $i=0,1,2, \ldots$ e cada $s \in\left[s_{i}, s_{i+1}\right]$, temos

$$
\begin{gathered}
\sum_{i=0}^{m-1}\left|\int_{s_{i}}^{s_{i+1}}\left[f_{0}\left(y_{s}\right)-f_{0}\left(y_{s_{i}}\right)\right] d s\right| \leq \sum_{i=0}^{m-1} \int_{s_{i}}^{s_{i+1}}\left|f_{0}\left(y_{s}\right)-f_{0}\left(y_{s_{i}}\right)\right| d s \leq \\
\leq C \sum_{i=0}^{m-1} \int_{s_{i}}^{s_{i+1}}\left\|y_{s}-y_{s_{i}}\right\| d s \leq C \sum_{i=0}^{m-1} \sup _{\sigma \in\left[t_{i}, t_{i+1}\right]}\left\|y_{\sigma}-y_{t_{i}}\right\| 2 \delta\left(\tau_{i}\right)<C \frac{\eta}{\max (N, C) 2^{i+1}} \varepsilon
\end{gathered}
$$

que tende a zero quando $\varepsilon \rightarrow 0^{+}$. Assim, o segundo somando ao lado direito de (4.23) tende a zero quando $\varepsilon \rightarrow 0^{+}$. 
Finalmente,

$$
\sum_{i=0}^{m-1}\left|\int_{s_{i}}^{s_{i+1}} f_{1}\left(y_{s_{i}}, \frac{s}{\varepsilon}\right) d s\right|
$$

pode ser feito tão pequeno quanto se queira pelo Corolário 4.5, e então, o terceiro somando ao lado direito de (4.23) tende a zero quando $\varepsilon \rightarrow 0^{+}$. De fato,

$$
\begin{gathered}
\sum_{i=0}^{m-1}\left|\int_{s_{i}}^{s_{i+1}} f_{1}\left(y_{s_{i}}, \frac{s}{\varepsilon}\right) d s\right|=\sum_{i=0}^{m-1}\left|\int_{s_{i}}^{s_{i+1}}\left[f\left(y_{s_{i}}, \frac{s}{\varepsilon}\right)-f_{0}\left(y_{s_{i}}\right)\right] d s\right|= \\
=\sum_{i=0}^{m-1}\left|\int_{s_{i}}^{s_{i}+\alpha_{i}}\left[f\left(y_{s_{i}}, \frac{s}{\varepsilon}\right)-f_{0}\left(y_{s_{i}}\right)\right] d s\right|=\sum_{i=0}^{m-1} \alpha_{i}\left|\frac{\varepsilon}{\alpha_{i}} \int_{s_{i} / \varepsilon}^{s_{i} / \varepsilon+\alpha_{i} / \varepsilon} f\left(y_{s_{i}}, s\right) d s-f_{0}\left(y_{s_{i}}\right)\right|,
\end{gathered}
$$

onde $\alpha_{i}=s_{i+1}-s_{i}$, para todo $i=0,1,2, \ldots$

Agora, para cada $i=0,1,2, \ldots$, seja

$$
\beta_{i}=\frac{\varepsilon}{\alpha_{i}} \int_{s_{i} / \varepsilon}^{s_{i} / \varepsilon+\alpha_{i} / \varepsilon} f\left(y_{s_{i}}, s\right) d s-f_{0}\left(y_{s_{i}}\right)
$$

e seja $\beta=\max \left\{\beta_{i} ; i=0,1,2, \ldots\right\}$.

Pelo Corolário 4.5, $\beta$ pode ser tomado suficientemente pequeno. Portanto

$$
\left|\sum_{i=0}^{m-1} \alpha_{i} \beta_{i}\right| \leq \beta \sum_{i=0}^{m-1} \alpha_{i}=\beta \sum_{i=0}^{m-1}\left(s_{i+1}-s_{i}\right)=\beta t .
$$

Com isto, o terceiro somando ao lado direito de (4.23) tende a zero quando $\varepsilon \rightarrow 0^{+}$e terminamos a prova.

Para apresentarmos nossa versão do Princípio da Média para EDFRs, vamos precisar impor uma condição sobre $f$ bastante forte. Vamos precisar que a função $f(\psi, t)$ seja lipschitziana na primeira variável. Consideremos, então, a seguinte condição:

$(K)$ existe uma constante positiva $K$ tal que, para $\psi, \varphi \in G^{-}\left([-r, 0], \mathbb{R}^{n}\right)$ e $u \in[0,+\infty)$,

$$
|f(\psi, u)-f(\varphi, u)| \leq K\|\psi-\varphi\|
$$

Note que se $f$ satisfizer a condição $(K)$, então $f$ também satisfará as condições $\left(\mathcal{B}^{*}\right)$ e $\left(\mathcal{C}^{*}\right)$.

Observação 4.7. Obviamente que poderíamos ter considerado a condição de Lipschitz acima como sendo local. Alternativamente, poderíamos ter considerado $f(\psi, t)$ como sendo uniformemente continua em $\psi$, com respeito a $t \in[0,+\infty)$. 
Teorema 4.8. Consideremos a EDFR (4.16), onde $\phi \in G^{-}\left([-r, 0], \mathbb{R}^{n}\right)$ e f satisfaz a condição (K). Consideremos, também, a EDFR média (4.20), onde $f_{0}$ é dada por (4.17). Sejam $[0, \bar{b})$ e $[0, b)$ os intervalos maximais de existência de (4.16) e (4.20) respectivamente, e sejam $x^{\varepsilon}$ solução maximal de (4.16) e y solução maximal de (4.20). Seja $M>0$, $M \leq \min (\bar{b}, b)$. Então, para todo $t \in[0, M]$, vale

$$
\lim _{\varepsilon \rightarrow 0+}\left|x^{\varepsilon}(t)-y(t)\right|=0
$$

Prova: Para cada $t \in[0, L]$, valem as igualdades

$$
x^{\varepsilon}(t)=\phi(0)+\int_{0}^{t} f\left(\left(x^{\varepsilon}\right)_{s}, \frac{s}{\varepsilon}\right) d s \quad \text { e } \quad y(t)=\phi(0)+\int_{0}^{t} f_{0}\left(y_{s}\right) d s .
$$

Então, usando a condição $(K)$, temos

$$
\begin{gathered}
\left|x^{\varepsilon}(t)-y(t)\right|=\left|\int_{0}^{t}\left[f\left(\left(x^{\varepsilon}\right)_{s}, \frac{s}{\varepsilon}\right)-f_{0}\left(y_{s}\right)\right] d s\right| \leq \\
\leq \int_{0}^{t}\left|f\left(\left(x^{\varepsilon}\right)_{s}, \frac{s}{\varepsilon}\right)-f\left(y_{s}, \frac{s}{\varepsilon}\right)\right| d s+\left|\int_{0}^{t}\left[f\left(y_{s}, \frac{s}{\varepsilon}\right)-f_{0}\left(y_{s}\right)\right] d s\right| \leq \\
\leq K \int_{0}^{t}\left\|\left(x^{\varepsilon}\right)_{s}-y_{s}\right\| d s+\left|\int_{0}^{t}\left[f\left(y_{s}, \frac{s}{\varepsilon}\right)-f_{0}\left(y_{s}\right)\right] d s\right|
\end{gathered}
$$

Usando o fato de que $\left(x^{\varepsilon}\right)_{0}=\phi=y_{0}$, temos

$$
\left\|\left(x^{\varepsilon}\right)_{s}-y_{s}\right\|=\sup _{\theta \in[-r, 0]}\left|x^{\varepsilon}(s+\theta)-y(s+\theta)\right|=\sup _{\sigma \in[0, s]}\left|x^{\varepsilon}(\sigma)-y(\sigma)\right|
$$

e, portanto,

$$
\left|x^{\varepsilon}(t)-y(t)\right| \leq K \int_{0}^{t} \sup _{\sigma \in[0, s]}\left|x^{\varepsilon}(\sigma)-y(\sigma)\right| d s+\left|\int_{0}^{t}\left[f\left(y_{s}, \frac{s}{\varepsilon}\right)-f_{0}\left(y_{s}\right)\right] d s\right| .
$$

Como o lado direito de (4.26) é crescente, então vale

$$
\sup _{\tau \in[0, t]}\left|x^{\varepsilon}(\tau)-y(\tau)\right| \leq K \int_{0}^{t} \sup _{\sigma \in[0, s]}\left|x^{\varepsilon}(\sigma)-y(\sigma)\right| d s+\sup _{\tau \in[0, t]}\left|\int_{0}^{\tau}\left[f\left(y_{s}, \frac{s}{\varepsilon}\right)-f_{0}\left(y_{s}\right)\right] d s\right| .
$$

Daí, pela desigualdade de Gronwall, obtemos

$$
\sup _{\tau \in[0, t]}\left|x^{\varepsilon}(\tau)-y(\tau)\right| \leq e^{K t} \sup _{\tau \in[0, t]}\left|\int_{0}^{\tau}\left[f\left(y_{s}, \frac{s}{\varepsilon}\right)-f_{0}\left(y_{s}\right)\right] d s\right| .
$$


Finalmente,

$$
\sup _{\tau \in[0, t]}\left|\int_{0}^{\tau}\left[f\left(y_{s}, \frac{s}{\varepsilon}\right)-f_{0}\left(y_{s}\right)\right] d s\right|
$$

pode ser tomado suficientemente pequeno, pelo Lema 4.6 e pela compacidade do intervalo $[0, t]$. Assim, terminamos a prova.

\subsection{O Princípio da Média para EDFRIs}

A fim de obtermos o Princípio da Média para EDFRIs, vamos considerar a situação na qual obtivemos o Princípio da Média para EDFRs e vamos adicionar efeitos impulsivos em tempo pré fixado. A partir daí, vamos considerar, também, EDOGs e vamos utilizar a correspondência entre EDFRIs e EDOGs e o Princípio da Média para EDOGs para obtermos nosso resultado.

Primeiramente, faremos algumas observações.

Consideremos o sistema diferencial sem impulsos

$$
\left\{\begin{array}{l}
\dot{y}=f\left(y_{t}, t\right) \\
y_{0}=\phi
\end{array}\right.
$$

onde $\phi \in G^{-}\left([-r, 0], \mathbb{R}^{n}\right)$ e $f$ satisfaz as condições $\left(A^{*}\right)$ e $(K)$. Consideremos, também, sua EDOG correspondente

$$
\frac{d x}{d \tau}=D F(x, t)
$$

onde $F: \Omega \rightarrow P C_{1}$ é dada da seguinte forma: para cada $y \in P C_{1}$ e cada $t \geq-r$,

$$
F(y, t)(\vartheta)=\left\{\begin{array}{l}
0,-r \leq \vartheta \leq 0 \text { ou }-r \leq t \leq 0 \\
\int_{0}^{\vartheta} f\left(y_{s}, s\right) d s, \quad 0 \leq \vartheta \leq t<\infty \\
\int_{0}^{t} f\left(y_{s}, s\right) d s, \quad 0 \leq t \leq \vartheta<\infty
\end{array}\right.
$$

e consideremos a condição inicial

$$
x(0)(\vartheta)= \begin{cases}\phi(\vartheta), & 0-r \leq \vartheta \leq 0, \\ \phi(0), & 0 \leq \vartheta<\infty .\end{cases}
$$

Seja $\varepsilon>0$ um parâmetro pequeno. Consideremos a EDFR

$$
\left\{\begin{array}{l}
\dot{y}=\varepsilon f\left(y_{t}, t\right) \\
y_{0}=\phi
\end{array}\right.
$$


Então a EDOG correspondente a (4.29) é dada por

$$
\frac{d x}{d \tau}=D[\varepsilon F(x, t)]
$$

com condição inicial (4.28).

Note que

$$
\varepsilon F\left(y, \frac{t}{\varepsilon}\right)\left(\frac{\vartheta}{\varepsilon}\right)= \begin{cases}0,-r \leq \vartheta \leq 0 \text { ou }-r \leq t \leq 0, \\ \varepsilon \int_{0}^{\vartheta / \varepsilon} f\left(y_{s}, s\right) d s, & 0 \leq \frac{\vartheta}{\varepsilon} \leq \frac{t}{\varepsilon}<\infty \\ \varepsilon \int_{0}^{t / \varepsilon} f\left(y_{s}, s\right) d s, & 0 \leq \frac{t}{\varepsilon} \leq \frac{\vartheta}{\varepsilon}<\infty\end{cases}
$$

Seja $y$ a solução de (4.29). Fazendo a mudança de variável $\varphi(s)=\frac{s}{\varepsilon}$, temos

$$
\int_{0}^{t / \varepsilon} \varepsilon f\left(y_{s}, s\right) d s=\int_{0}^{t} f\left((y)_{\frac{s}{\varepsilon}}, \frac{s}{\varepsilon}\right) d s=\int_{0}^{t} f\left(\zeta_{s}, \frac{s}{\varepsilon}\right) d s,
$$

onde $(\zeta)_{\varepsilon t}=(y)_{t}, t \in\left[0, \frac{L}{\varepsilon}\right]$, isto é, $\zeta$ é solução, em $[0, L]$, de

$$
\left\{\begin{array}{l}
\dot{y}=f\left(y_{t}, \frac{t}{\varepsilon}\right) \\
y_{0}=\phi
\end{array}\right.
$$

Por outro lado, considerando $f_{0}: G^{-}\left([-r, 0], \mathbb{R}^{n}\right) \rightarrow \mathbb{R}^{n}$ dada por

$$
f_{0}(\psi)=\lim _{T \rightarrow \infty} \frac{1}{T} \int_{0}^{T} f(\psi, s) d s
$$

e considerando a EDF autônoma média

$$
\left\{\begin{array}{l}
\dot{y}=f_{0}\left(y_{t}\right) \\
y_{0}=\phi
\end{array}\right.
$$

se $\bar{y}$ for solução de (4.33) em [0,L], então pelo Lema 4.6, teremos

$$
\lim _{\varepsilon \rightarrow 0} \int_{0}^{t} f\left(\bar{y}_{s}, \frac{s}{\varepsilon}\right) d s=\int_{0}^{t} f_{0}\left(\bar{y}_{s}\right) d s, \quad t \in[0, L] .
$$

Daí, usando (4.30) e (4.34), obtemos a seguinte aproximação

$$
\left|\varepsilon \int_{0}^{\frac{t}{\varepsilon}} f\left(y_{s}, s\right) d s-\int_{0}^{t} f_{0}\left(\bar{y}_{s}\right) d s\right| \approx\left|\int_{0}^{t} f\left(\zeta_{s}, \frac{s}{\varepsilon}\right) d s-\int_{0}^{t} f\left(\bar{y}_{s}, \frac{s}{\varepsilon}\right) d s\right|,
$$


para $\varepsilon>0$ suficientemente pequeno.

Usando a condição $(K)$, temos

$$
\left|\int_{0}^{t} f\left(\zeta_{s}, \frac{s}{\varepsilon}\right) d s-\int_{0}^{t} f\left(\bar{y}_{s}, \frac{s}{\varepsilon}\right) d s\right| \leq K \int_{0}^{t}\left\|\zeta_{s}-\bar{y}_{s}\right\| d s .
$$

Então, pelo Princípio da Média para EDFRs (Teorema 4.8), podemos concluir que o lado direito de (4.35) pode ser feito suficientemente pequeno. Assim, acabamos de mostrar o lema seguinte.

Lema 4.9. Consideremos as EDFRs (4.29) e (4.33). Então para todo $\rho>0$ e todo $L>0$, existe um $\varepsilon_{0}>0$ tal que para $\varepsilon \in\left(0, \varepsilon_{0}\right)$, vale

$$
\left|\varepsilon \int_{0}^{\frac{t}{\varepsilon}} f\left(y_{s}, s\right) d s-\int_{0}^{t} f_{0}\left(\bar{y}_{s}\right) d s\right|<\rho, \quad t \in[0, L],
$$

onde y é solução de (4.27) em $\left[0, \frac{L}{\varepsilon}\right]$ e $\bar{y}$ é solução de (4.33) em $[0, L]$.

Vamos prosseguir com nossas observações.

Definindo para $y \in P C_{1}$ e $t \geq-r$,

$$
H_{0}(y, t)(\vartheta)=\left[F_{0}(y) t\right](\vartheta)=\left\{\begin{array}{l}
0,-r \leq \vartheta \leq 0 \text { or }-r \leq t \leq 0, \\
\int_{0}^{\vartheta} f_{0}\left(y_{s}\right) d s, \quad 0 \leq \vartheta \leq t<\infty \\
\int_{0}^{t} f_{0}\left(y_{s}\right) d s, \quad 0 \leq t \leq \vartheta<\infty
\end{array}\right.
$$

temos, pelo Lema 4.9,

$$
F_{0}(y) t=\lim _{\varepsilon \rightarrow 0^{+}} \varepsilon F\left(y, \frac{t}{\varepsilon}\right)=\lim _{\varepsilon \rightarrow 0^{+}} t \frac{\varepsilon}{t} F\left(y, \frac{t}{\varepsilon}\right) .
$$

Portanto

$$
F_{0}(y)=\lim _{\varepsilon \rightarrow 0^{+}} \frac{\varepsilon}{t} F\left(y, \frac{t}{\varepsilon}\right)
$$

ou, equivalentemente,

$$
F_{0}(y)=\lim _{T \rightarrow \infty} \frac{F(y, T)}{T} .
$$

Note que (4.36) define a EDO generalizada

$$
\frac{d x}{d \tau}=D\left[F_{0}(y) t\right]
$$

que corresponde à EDFR média (4.33). 
Por outro lado, como comentamos anteriormente, (4.37) é na verdade uma EDO em espaços de Banach $\left(\mathrm{em} G^{-}([-r, \infty))\right)$ e pode ser escrita como

$$
\dot{x}=F_{0}(x)
$$

Agora, consideremos as seguintes EDFRs com impulsos

$$
\left\{\begin{array}{l}
\dot{y}=f\left(y_{t}, t\right), t \neq t_{i} \\
\Delta x\left(t_{i}\right)=I_{i}\left(x\left(t_{i}\right)\right), \quad i=1,2, \ldots \\
y_{0}=\phi
\end{array}\right.
$$

e

$$
\left\{\begin{array}{l}
\dot{y}=\varepsilon f\left(y_{t}, t\right), t \neq t_{i} \\
\Delta x\left(t_{i}\right)=\varepsilon I_{i}\left(x\left(t_{i}\right)\right), \quad i=1,2, \ldots \\
y_{0}=\phi
\end{array}\right.
$$

onde $\phi \in G^{-}\left([-r, 0], \mathbb{R}^{n}\right)$ e $f: P C_{1} \times[0, \infty) \rightarrow \mathbb{R}^{n}$ satisfaz as condições $(A)$ e $(K)$.

Seja

$$
I^{0}(x)=\lim _{T \rightarrow \infty} \frac{1}{T} \sum_{0 \leq t_{i}<T} I_{i}(x), \quad x \in \mathbb{R}^{n}
$$

Então

$$
I^{0}\left(y\left(t_{i}\right)\right)=\lim _{T \rightarrow \infty} \frac{1}{T} \sum_{0 \leq t_{i}<T} I_{i}\left(y\left(t_{i}\right)\right)=\lim _{T \rightarrow \infty} \frac{1}{T} \sum_{i=0}^{\infty} I_{i}\left(y\left(t_{i}\right)\right) H_{t_{i}}(T),
$$

onde $H_{t_{i}}$ é a função Heaviside contínua à esquerda concentrada em $t_{i}$

Para $y \in P C_{1}$ e $t \in[-r, \infty)$, sejam

$$
\begin{gathered}
J(y, t)(\vartheta)=\sum_{i=0}^{\infty} H_{t_{i}}(t) H_{t_{i}}(\vartheta) I_{i}\left(y\left(t_{i}\right)\right) \quad \mathrm{e} \\
{\left[J_{0}(y) t\right](\vartheta)=\sum_{i=0}^{\infty} H_{t_{i}}(t) H_{t_{i}}(\vartheta) I^{0}\left(y\left(t_{i}\right)\right)}
\end{gathered}
$$

onde $\vartheta \in[-r, \infty)$. Então

$$
J_{0}(y)=\lim _{T \rightarrow \infty} \frac{J(y, T)}{T} .
$$

Vamos considerar que as condições $\left(A^{\prime}\right)$ e $\left(B^{\prime}\right)$ estejam satisfeitas.

O teorema que apresentaremos a seguir é o principal resultado deste trabalho. Ele é uma versão do Princípio da Média para EDFRIs e trata-se de um resultado inédito. 
Teorema 4.10. Sejam y e $y^{\varepsilon}$ respectivamente as soluções das EDFRIs (4.38) e (4.39) em $\left[0, \frac{L}{\varepsilon}\right]$, onde $\phi \in G^{-}\left([-r, 0], \mathbb{R}^{n}\right)$ e $f: P C_{1} \times[0, \infty) \rightarrow \mathbb{R}^{n}$ satisfaz as condições $(A) e$ (K). Suponhamos que

$$
\lim _{T \rightarrow \infty} \frac{1}{T} \int_{\alpha}^{T+\alpha} M(s) d s \leq c, \quad c=\text { constante }
$$

para todo $\alpha \geq 0$. Seja $0 \leq t_{1}<t_{2}<\ldots<t_{k}<\ldots$, com $t_{k} \rightarrow \infty$ quando $k \rightarrow \infty$, uma sequência de pontos tal que

$$
\limsup _{T \rightarrow \infty} \sum_{\alpha \leq t_{i} \leq \alpha+T} 1 \leq d
$$

para todo $\alpha \geq 0$ e suponhamos que $I_{i}: \mathbb{R}^{n} \rightarrow \mathbb{R}^{n}, i=0,1,2, \ldots$, seja uma sequência de operadores de impulso satisfazendo as condições $\left(A^{\prime}\right)$ e $\left(B^{\prime}\right)$. Suponhamos, ainda, que

$$
\lim _{T \rightarrow \infty} \frac{1}{T} \sum_{0 \leq t_{i}<T} I_{i}(x)=I_{0}(x), \quad x \in \mathbb{R}^{n} .
$$

Então para todo $\mu>0$ e todo $L>0$, existe um $\varepsilon_{0}>0$ tal que para $\varepsilon \in\left(0, \varepsilon_{0}\right)$, a desigualdade

$$
\left\|\left(y^{\varepsilon}\right)_{t}-\left(\bar{y}^{\varepsilon}\right)_{t}\right\|<\mu
$$

acontece, onde $\bar{y}^{\varepsilon}$ é a solução em $\left[0, \frac{L}{\varepsilon}\right]$ da EDFR com impulsos

$$
\left\{\begin{array}{l}
\dot{y}=\varepsilon f_{0}\left(y_{t}\right), t \neq t_{i} \\
\Delta y\left(t_{i}\right)=\varepsilon I^{0}\left(y\left(t_{i}\right)\right), \quad i=1,2, \ldots \\
y_{0}=\phi
\end{array}\right.
$$

Prova: Primeiramente, observemos que o sistema (4.39) é equivalente à EDOG

$$
\frac{d x}{d \tau}=D[\varepsilon G(x, t)]
$$

com condição inicial (4.28), onde $G$ é dada por (3.18), e pelo Teorema 3.2, sua solução $x_{\varepsilon}$ é dada por

$$
x_{\varepsilon}(t)(\vartheta)=\left\{\begin{array}{l}
y^{\varepsilon}(\vartheta), \vartheta \in[-r, t] \\
y^{\varepsilon}(t), \vartheta \in[t, \infty) .
\end{array}\right.
$$

Conforme o que foi visto anteriormente, $G \in \mathcal{F}(\Omega, h)$, se tomarmos, por exemplo,

$$
h(t)=\left\{\begin{array}{l}
\int_{0}^{t} M(s) d s+K, \quad t \geq 0 \\
0,-r \leq t \leq 0
\end{array}\right.
$$


Pelo Teorema 3.2, também é verdade que $\xi_{\varepsilon}$ dada por

$$
\xi_{\varepsilon}(t)(\vartheta)=\left\{\begin{array}{l}
\left(\bar{y}^{\varepsilon}\right)(\vartheta), \vartheta \in[-r, t] \\
\left(\bar{y}^{\varepsilon}\right)(t), \vartheta \in[t, \infty) .
\end{array}\right.
$$

é solução de

$$
\frac{d x}{d \tau}=D\left[\varepsilon G_{0}(x)\right]
$$

Pelas observações anteriores ao teorema, se

$$
G(x, t)=F(x, t)+J(x, t) \quad \text { e } \quad G_{0}(x)=F_{0}(x)+J_{0}(x),
$$

então

$$
\lim _{r \rightarrow \infty} \frac{G(x, r)(\vartheta)}{r}=G_{0}(x)(\vartheta) .
$$

Observemos, também, que para todo $\alpha>0$, vale

$$
\begin{aligned}
\lim _{T \rightarrow \infty} \frac{h(T+\alpha)-h(\alpha)}{T} & =\lim _{T \rightarrow \infty} \frac{h_{2}(T+\alpha)-h_{2}(\alpha)+h_{1}(T+\alpha)-h_{1}(\alpha)}{T}= \\
& =\lim _{T \rightarrow \infty} \frac{1}{T} \int_{\alpha}^{T+\alpha} M(s) d s+\frac{1}{T} \int_{\alpha}^{T+\alpha} K d s+ \\
& +\lim _{T \rightarrow \infty} \frac{1}{T}\left(\max \left(K_{1}, K_{2}\right) \sum_{i=0}^{\infty}\left[H_{t_{i}}(T+\alpha)-H_{t_{i}}(\alpha)\right]\right) \\
& \leq c+K+\max \left(K_{1}, K_{2}\right) d,
\end{aligned}
$$

Então todas as hipóteses do Teorema 4.1 estão satisfeitas e, portanto, para quaisquer $\mu>0$ e $L>0$, existe $\varepsilon_{0}>0$ tal que para $\varepsilon \in\left(0, \varepsilon_{0}\right)$, vale a desigualdade

$$
\left\|x_{\varepsilon}(t)-\xi_{\varepsilon}(t)\right\|<\mu
$$

para $t \in\left[0, \frac{L}{\varepsilon}\right]$. Finalmente, para todo $t \in\left[0, \frac{L}{\varepsilon}\right]$, temos

$$
\begin{aligned}
& \left\|\left(y^{\varepsilon}\right)_{t}-\left(\bar{y}^{\varepsilon}\right)_{t}\right\|=\sup _{\theta \in[-r, 0]}\left|y^{\varepsilon}(t+\theta)-\bar{y}^{\varepsilon}(t+\theta)\right|=\sup _{\vartheta \in[t-r, t]}\left|y^{\varepsilon}(\vartheta)-\bar{y}^{\varepsilon}(\vartheta)\right| \leq \\
\leq & \sup _{\vartheta \in[-r, t]}\left|y^{\varepsilon}(\vartheta)-\bar{y}^{\varepsilon}(\vartheta)\right|=\sup _{\vartheta \in[-r, t]}\left|x_{\varepsilon}(t)(\vartheta)-\xi_{\varepsilon}(t)(\vartheta)\right| \leq\left\|x_{\varepsilon}(t)-\xi_{\varepsilon}(t)\right\|<\mu
\end{aligned}
$$

e obtemos o resultado desejado. 


\section{Referências Bibliográficas}

[1] S. M. Afonso, E. M. Bonotto, M. Federson, S. Schwabik, Discontinuous local semiflows for Kurzweil equations and LaSalle's Invariance Principle, pré-print.

[2] Z. Artstein, Topological dynamics of an ordinary differential equation and Kurzweil equations, J. Diff. Eq., 23 (1977), 224-243.

[3] D. D. Bainov, S. D. Milusheva, Justification of the averaging method for a system of functional differential equations with variable structure and impulses, Appl. Math. and Optimization, 16 (1987), 19-36.

[4] N. N. Bogolyubov, A. Mitropolskii, Assymptotic Methods in the Theory of Non-linear Oscillations, Moscou, Nauka, 1963 (em russo).

[5] M. Federson, Š. Schwabik, Stability for retarded functional differential equations, Ukrainian Math J., 244 (2008), 2334-2349.

[6] M. Federson, Š. Schwabik, A new approach to impulsive retarded differential equations: stability results, Functional Differential Equations, em fase de publiçao.

[7] M. Federson, Š. Schwabik, Generalized ODEs approach to impulsive retarded functional differential equations, Differential and Integral Equations., 19 (11), (2006), 1201-1234.

[8] V. I. Foduck, The method of averaging for differential difference equations of the neutral type, Ukrai. Mat. Zh., 20 (1968), 203-209.

[9] M. Frasson, Sistemas Impulsivos do Ponto de Vista das Equações Diferenciais em Medida, Dissertação de Mestrado, ICMC-USP, 2000.

[10] L. P. Gimenes, Estabilidade e Oscilação de Soluções de Equações Diferenciais com Retardos e Impulsos, Tese de Doutorado, ICMC-USP, 2007. 
[11] J. B. Godoy, M. Federson, Averaging for impulsive functional differential equations: a new approach, pré-print.

[12] A. Halanay, The method of averaging in equations with retardation, Rev. Mat. Pur. Appl. Acad. R.P.R., 4 (1959), 467-483.

[13] J. K. Hale; S. M. V. Lunel, Introduction to Functional Differential Equations, Springer-Verlag, New York, 1993.

[14] J. K. Hale, Averaging methods for differntial equations with retarded arguments with a small parameter, J. Diff. Eq., 2 (1966), 57-73.

[15] J. K. Hale, S. M. Verduyn Lunel, Averaging in infinite dimensions, J. of Integral Eq. and Appl., 2 (1990), 463-494.

[16] C. S. Hoing Volterra Stieljes Integral Equations, North Holland Publ. Comp., Amsterdam, 1975.

[17] N. N. Krylov, N. N. Bogolyubov, New Methods in Linear Mechanics, Kiev, GTTs, 1934 (em russo).

[18] J. Kurzweil, Generalized ordinary differential equations and continuous dependence on a parameter, Czech. Math. J., 7 (82), (1957), 418-448.

[19] M. Lakrib, Time Averaging for functional differential equations, Journal of Applied Mathematics, 1 (2003), 1-16.

[20] M. Lakrib, T. Sari, Averaging results for functional differential equations, Siberian Math. J. 45 (2), (2004), 311-320

[21] V. Lakshmikantham; D. D. Bainov; P. S. Simeonov, Theory of Impulsive Differential Equations, World Scientific, Singapore, 1989.

[22] B. Lehman, S. P. Weibel, Averaging theory for functional differential equations, Proc. 37th IEEE Conference on Decision and Control, USA, (1998), 1352-1357.

[23] X. Liu; G. Ballinger, Existence and uniqueness results for impulsive delay differential equations, Dyn. Contin. Discrete Impuls. Systems, 5 (1999), 579-591.

[24] G. N. Medvedev, Asymptotic solutions of some systems of differential equations with deviating argument,Soviet Math. Dokl., 9 (1968), 85-87. 
[25] T. Sari, Averaging for ordinary ifferential equations and functional differential equations. The Strength of Nonstandard Analusys, 286-305, SpringerWienNewYork, Vienna, 2007.

[26] Š. Schwabik, Generalized Ordinary Differential Equations, World Scientific, Series in Real Anal., vol. 5, 1992.

[27] V. Strygin, The averaging principle for equations with hereditary. Ukrainian Math. J., 22(4), (1971), 430-439.

[28] V. M. Volosov, G. N. Medvedev, B. I. Morgunov, Mr 32-7904, Vestnik Moskov. Univ. Ser. III Fiz. Astronom., 6 (1965), 89.

[29] Xinzhi Liu, G. Ballinger, Continuous dependence on initial values for impulsive delay differential equations, Appl. Math. Letters, 17, (2004), 483-490. 